\title{
BIOCONVERSION PROCESS OF SOURCE-SEPARATED ORGANIC WASTE FOR ETHANOL PRODUCTION
}

by

Valeriy Bekmuradov

M. Eng., Ryerson University, Toronto, 2008

\author{
A dissertation \\ presented to Ryerson University \\ in partial fulfillment of the \\ requirements for the degree of \\ Doctor of Philosophy \\ in the Program of \\ Civil Engineering
}

Toronto, Ontario, Canada, 2015

(C) Valeriy Bekmuradov 2015 


\section{AUTHOR'S DECLARATION}

I hereby declare that I am the sole author of this dissertation. This is a true copy of the dissertation, including any required final revisions, as accepted by my examiners.

I authorize Ryerson University to lend this dissertation to other institutions or individuals for the purpose of scholarly research.

I further authorize Ryerson University to reproduce this dissertation by photocopying or by other means, in total or in part, at the request of other institutions or individuals for the purpose of scholarly research.

I understand that my dissertation may be made electronically available to the public. 


\title{
BIOCONVERSION PROCESS OF SOURCE-SEPARATED ORGANIC WASTE FOR ETHANOL PRODUCTION
}

\author{
Doctor of Philosophy \\ 2015 \\ Valeriy Bekmuradov \\ Civil Engineering, Ryerson University
}

\begin{abstract}
Production of biofuel such as ethanol from lignocellulosic biomass is a beneficial way to meet sustainability, energy security, and environmental goals. Lignocellulosic biomass such as source-separated organic (SSO) waste is particularly attractive since it is widely available, often at a negative cost, reduce the land depletion from using food-based biomass for ethanol production and reduce the amout of generated waste. Therefore, in order to meet the future fuel demands and cope with increasing volume of municipal waste this study was a first attempt to use SSO as a feedstock for ethanol production.

The main objectives of the study were: a) to compare standard and modified celluloseorganic-solvent-based lignocellulosic fractionation (COSLIF) pretreatment of SSO waste for ethanol production in terms of enzyme savings, sugar formation and ethanol yields; b) to produce ethanol from SSO by using modified COSLIF pretreatment and fermentation with two different recombinant strains: Z. mobilis 8b and S. cerevisiae DA2416; and c) to develop experimental kinetic model capable of predicting behavior of batch SSCF on SSO waste with different SSO substrate concentrations using Berkeley Madonna program.
\end{abstract}


Based on the obtained results, it was found that SSO is an excellent feedstock material for ethanol conversion. The efficiency of modified COSLIF pretreatment was improved by $20 \%$ compared to standard method using ethanol washing of pretreated SSO samples during the experimental procedures instead of acetone. On average, glucose yield from SSO samples pretreated by modified COSLIF was about $90 \%$ compared to $10 \%$ for untreated samples. S. cerevisiae DA2416 outperformed Z. mobilis 8b on ethanol yields during the fermentation process, with $0.50 \mathrm{~g}$ ethanol/g potential sugar fed on SSO in less than 5 days, with a 96\% cellulose conversion, totalling in $150 \mathrm{~g} / \mathrm{L}$ ethanol produced. A kinetic model with newly integrated values of experimentally defined SSO feedstock constants was proven to predict the ethanol yield accurately with substrate concentration ranges of $20 \mathrm{~g} / \mathrm{L}$ - $50 \mathrm{~g} / \mathrm{L}$. Model prediction at higher substrate concentration (e.g. $100 \mathrm{~g} / \mathrm{L}$ ) deviated from the experimental values, suggesting that ethanol inhibition is a major factor in bioethanol conversion. 


\section{ACKNOWLEDGEMENTS}

I would like to express my appreciation to my supervisor Professor Grace Luk for her guidance and encouragment as well as a giving me an opportunity to complete this work. I also would like to thank Doctors: Darko Joksimovic, James Li, and Jiangning Wu for serving on my thesis examination committee and their guidance, patience and support.

My sincere gratitude goes to all good friends of mine whom I had a pleasure to work with: Dr. Wai Yeung Yan, Dr. Alexandru Dimutrache, Robin Luong, Khurram Shahzad Baig, Mina Mirzajani, Benjamin Percy, Michael Faye, Mandana Ehsanipour and many others.

I would like to thank Dr. Gideon Wolfaartd and Dr. Hugh Lawford for their time and valuable advice to improve my work. I especially thank Miriam De Jong from the Chemistry and Biology department for her willingness to accommodate my experiments and tests in the biochemical laboratory. Also many thanks go to Shawn McFadden from Ryerson Analytical Centre for his assistance and help with HPLC set ups and runs. Special thanks to Mike Crupi from Optimum Waste Recycling Systems for providing SSO feedstock for this work.

Finally, I would like to thank my wife and family for their patience and support throughout this journey. 


\section{TABLE OF CONTENTS}

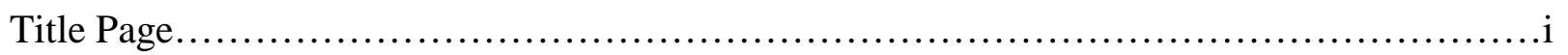

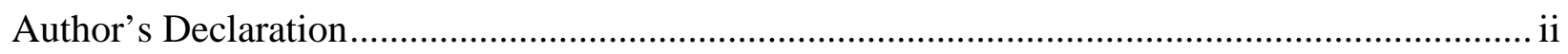

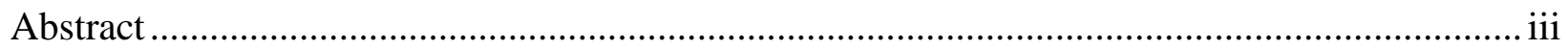

Acknowledgements .........................................................................................................

Table of Contents ............................................................................................................. vi

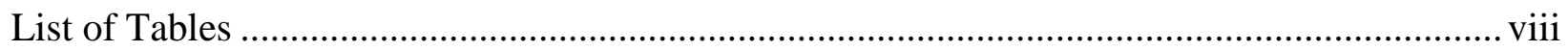

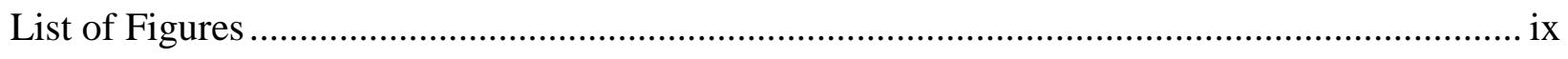

List of Appendices .............................................................................................................. X

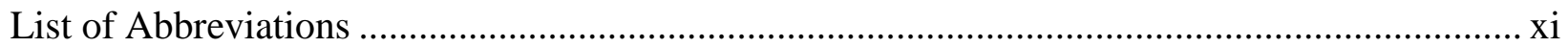

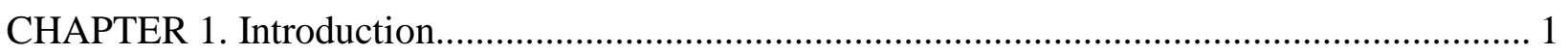

1.1. Biofuel from Lignocellulosic Conversion...................................................................... 1

1.2. Innovations in Biotechnology of Fuels and Cellulosic Feedstock Production ...................... 5

1.3. Source Separated Organic Waste as a Promising Cellulosic Feedstock ................................ 7

1.4. Ethanol Prediction Yields ................................................................................................. 10

1.5. Selection of Microorganisms ………………………….............................................. 11

1.6. Ethanol production and inhibitors ................................................................................ 14

1.7. Process Governing Factors......................................................................................... 19

CHAPTER 2. Thesis Objectives and Authorship ………………………………......................... 24

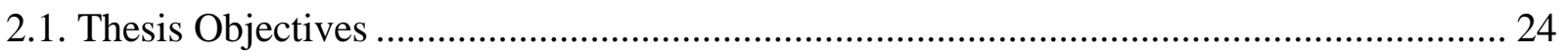

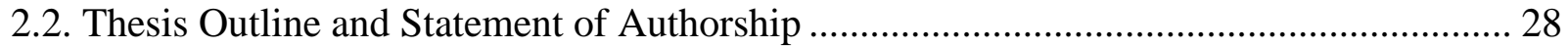

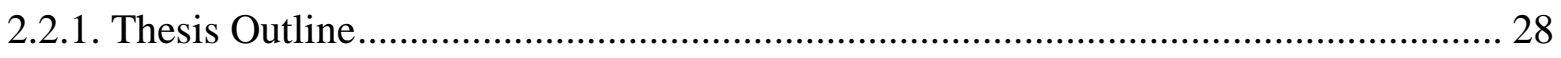

2.2.2. Statement of Authorship ........................................................................................ 32

CHAPTER 3. Improved Cellulose and Organic-Solvent-based Lignocellulosic Fractionation Pretreatment of Organic Waste for Bioethanol Production .......................................................... 34

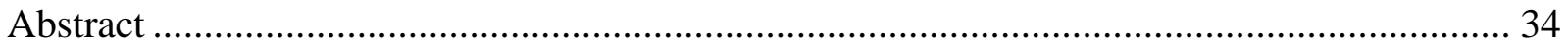

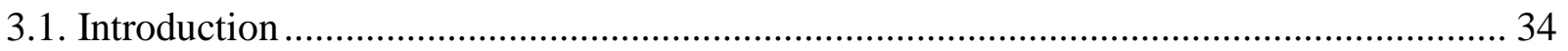

3.2. Materials and Methods ................................................................................................ 36

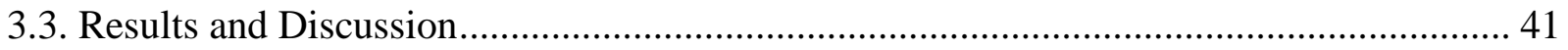




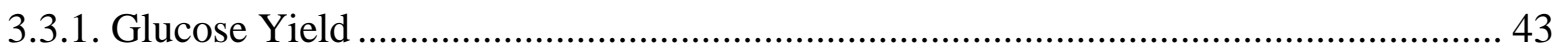

3.3.2. Enzymatic Hydrolysis ................................................................................................ 44

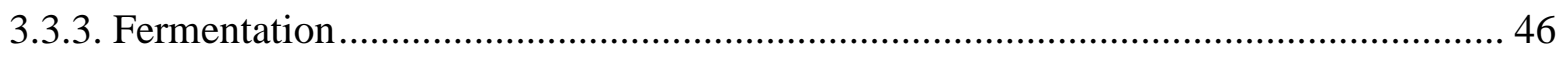

3.3.4. Comparison with Constructed Sugar Model .............................................................. 48

3.3.5. Qualitative Analysis .............................................................................................. 48

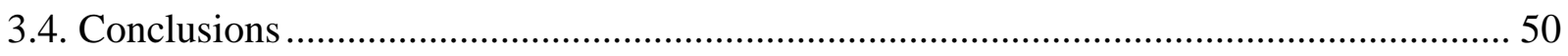

CHAPTER 4. Comparative Ethanol Productivities of Two Different Recombinant Fermenting Strains on Source-Separated Organic Waste ............................................................................... 52

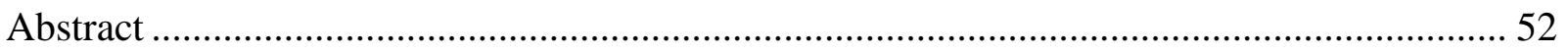

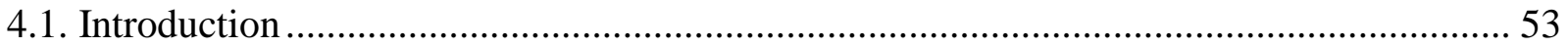

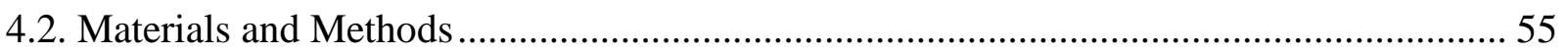

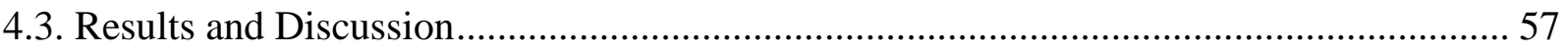

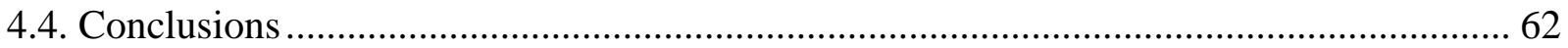

CHAPTER 5. Kinetic Model for Ethanol Production by Recombinant S. cerevisiae Strain on

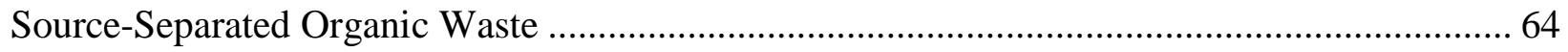

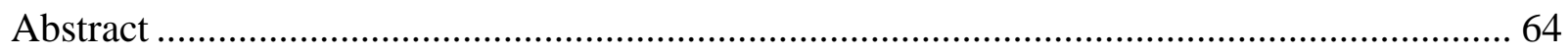

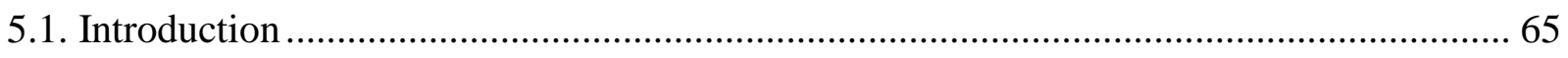

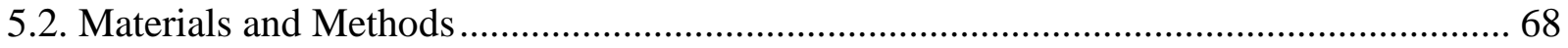

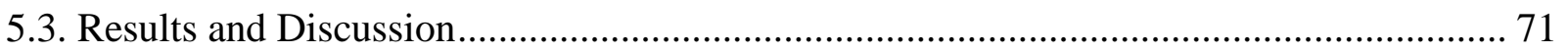

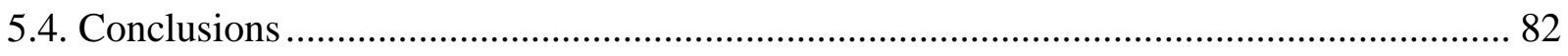

CHAPTER 6. Conclusions and Future Work Recommendations ................................................ 84

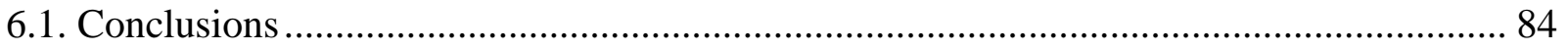

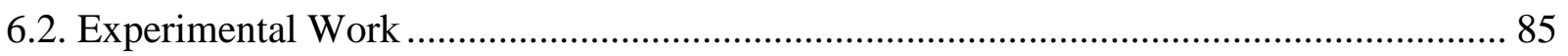

6.3. SSO Waste as a Lignocellulosic Feedstock for Ethanol Production..................................... 86

6.4. Comparative Ethanol Productivities of two Different Recombinant Fermenting Strains on

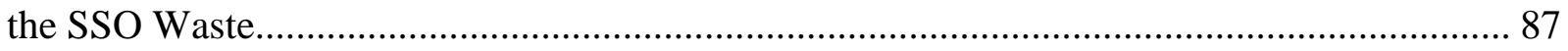

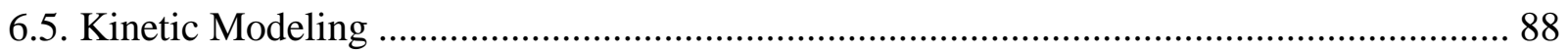

6.6. Future Work Recommendations. ...................................................................................... 88

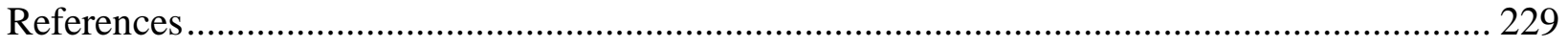




\section{LIST OF TABLES}

Table 1.1: Percent dry weight composition of lignocellulosic feedstocks.................................. 4

Table 1.2: Estimation of waste biomass production and amount of resources ........................... 6

Table 1.3: Classification of biomass resources................................................................. 9

Table 1.4: Selection of strains for paper sludge SSCF .................................................... 21

Table 3.1: Compositional analysis of source-separated organic samples ................................ 42

Table 4.1: Compositional analysis of SSO sample............................................................ 58

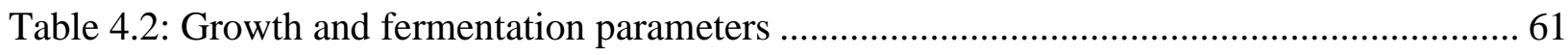

Table 5.1: Average parameter values for SSCF of the SSO …............................................ 74

Table 5.2: Sensitivity analysis of kinetic parameters for SSO used in this work ....................... 80

Table 5.3: Comparison performance of different $S$. cerevisiae strains.................................... 81 


\section{LIST OF FIGURES}

Figure 1.1: Lignocellulosic biomass constituents ......................................................... 2

Figure 1.2: Major steps in conversion of lignocellulosic biomass to ethanol............................... 3

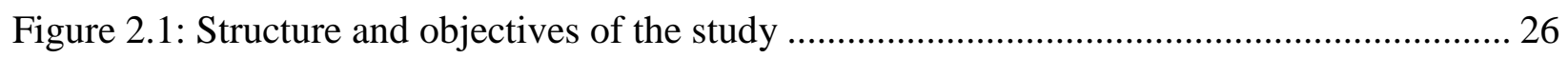

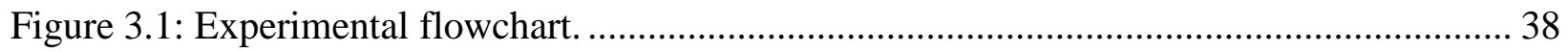

Figure 3.2: Glucose yields of standard and modified COSLIF pretreatment performed at $50^{\circ} \mathrm{C}$ for

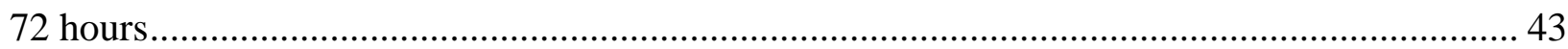

Figure 3.3: Time trend of glucose digestibility from the non-treated to standard and ............... 44

Figure 3.4: Ethanol concentration from modified COSLIF pretreated samples ......................... 47

Figure 3.5: Scanning electron microscopy images of source-separated organic waste............... 49

Figure 4.1: Glucan digestibility profiles for COSLIF treated and untreated SSO samples......... 59

Figure 4.2: Sugar consumption profiles of the SSO pretreated hydrolysates during fermentation

phase 60

Figure 4.3: Comparative fermentation performance of both strains for ethanol production in time

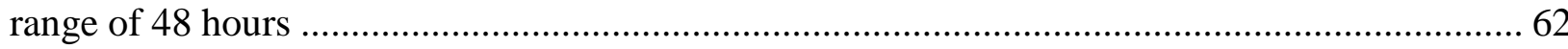

Figure 5.1: Cellulose adsorption data for pretreated SSO samples ........................................ 73

Figure 5.2: Experimental data and kinetic model prediction for SSO samples (substrate

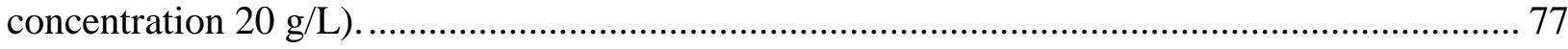

Figure 5.3: Experimental data and kinetic model prediction for SSO samples (substrate

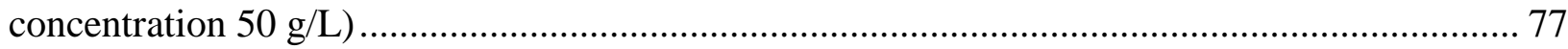

Figure 5.4: Experimental data and kinetic model prediction for SSO samples (substrate

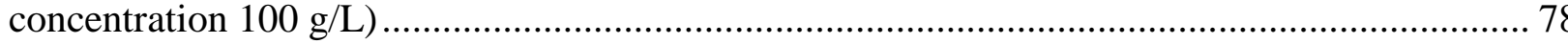




\section{LIST OF APPENDICES}

APPENDIX A: Glucose and ethanol yields calculations ................................................................ 90

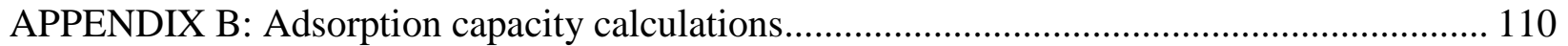

APPENDIX C: Berkeley Madonna computer program code and tabulated data ........................ 113

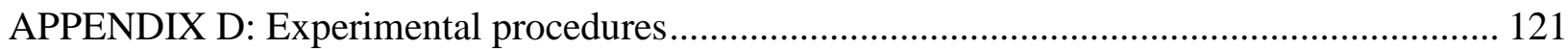

APPENDIX E: HPLC samples runs for enzymatic hydrolysis and fermentation ...................... 144 


\section{LIST OF ABBREVIATIONS}

\begin{tabular}{|c|c|}
\hline AIL & Acid Insoluble Lignin \\
\hline ASL & Acid Soluble Lignin \\
\hline ASTM & American Society for Testing and Materials \\
\hline ATS & Aufbereitungs Technology and System, AG \\
\hline $\mathrm{CBP}$ & Consolidated Bio-Processing \\
\hline COSLIF & Cellulose- and Organic Solvent-based Lignocellulosic Fractionation \\
\hline DP & Degree of Polymerization \\
\hline DSA & Dilute Sulfuric Acid \\
\hline EG & Endo-Glucanase \\
\hline FPU & Filter Paper Unit \\
\hline gdcw & Gram dry cell weight \\
\hline HMF & Hydroxymethylfurfural \\
\hline MSW & Municipal Solid Waste \\
\hline NREL & National Renewable Energy Laboratory \\
\hline ODW & Oven Dry Weight \\
\hline OFMSW & Organic Fraction of Municipal Solid Waste \\
\hline SEM & Scanning Electron Microscope \\
\hline SHF & Separate or Sequential Hydrolysis and Fermentation \\
\hline SSCF & Simultaneous Saccharification and Co-Fermentation \\
\hline SSO & Source-Separated Organic \\
\hline SWM & Solid Waste Management \\
\hline TAPPI & Technical Association of the Pulp and Paper Industry \\
\hline
\end{tabular}


TKN

TS

VFA

VOC

VS

EJ
Total Kjeldahl Nitrogen

\section{Total Solid}

Volatile Fatty Acid

Volatile Organic Compound

Volatile Solid

Exajoule 


\section{CHAPTER 1. INTRODUCTION}

\subsection{Biofuel from Lignocellulosic Conversion}

Over the last century as the world population has grown and more countries have become industrialized, energy consumption has increased steadily. Therefore, there is a great interest in exploring alternative energy sources. Unlike fossil fuel, ethanol is a renewable energy source produced via fermentation of sugars from various biomasses. Nowadays, ethanol is produced from both: food based biomass such as corn/sugarcane/wheat (Shen et al., 2012; Ferreira et al., 2014; Mohagheghi et al., 2015), and non-food based materials such as lignocellulosic biomass (Wyman and Yang, 2009; Zhao et al., 2012; Roberts et al., 2015).

Ethanol production using the current corn/starch based technology may not be practical because corn production for ethanol will compete with food for the limited agricultural land needed. Therefore, a potential source for low-cost ethanol is to utilize lignocellulosic biomass. Common examples of lignocellulosic biomass include energy crop, waste from agriculture and forest products, industries, and municipal waste. Unprocessed lignocellulosic biomass typically contains the order of 35\%-50\% cellulose, 20\%-35\% hemicellulose, and 15\%-20\% lignin plus extractives and ash (Wyman, 1999).

Figure 1.1 shows main constituents of lignocellulosic biomass in which cellulose is the primary component. The backbone of cellulose consists of D-glucose subunits bonded by $\beta-1-4$ linkages. This structure is stabilized by inter-chain hydrogen bonding between the G-3 hydroxyl and the oxygen in the pyranose ring. Hemicellulose, on the other hand, consists of branched chains of several sugar subunits such as xylose, mannose, arabonose, and galactose. It bonds to the cellulose and lignin molecules through covalent and hydrogen bonds (Fan et al., 1987). 


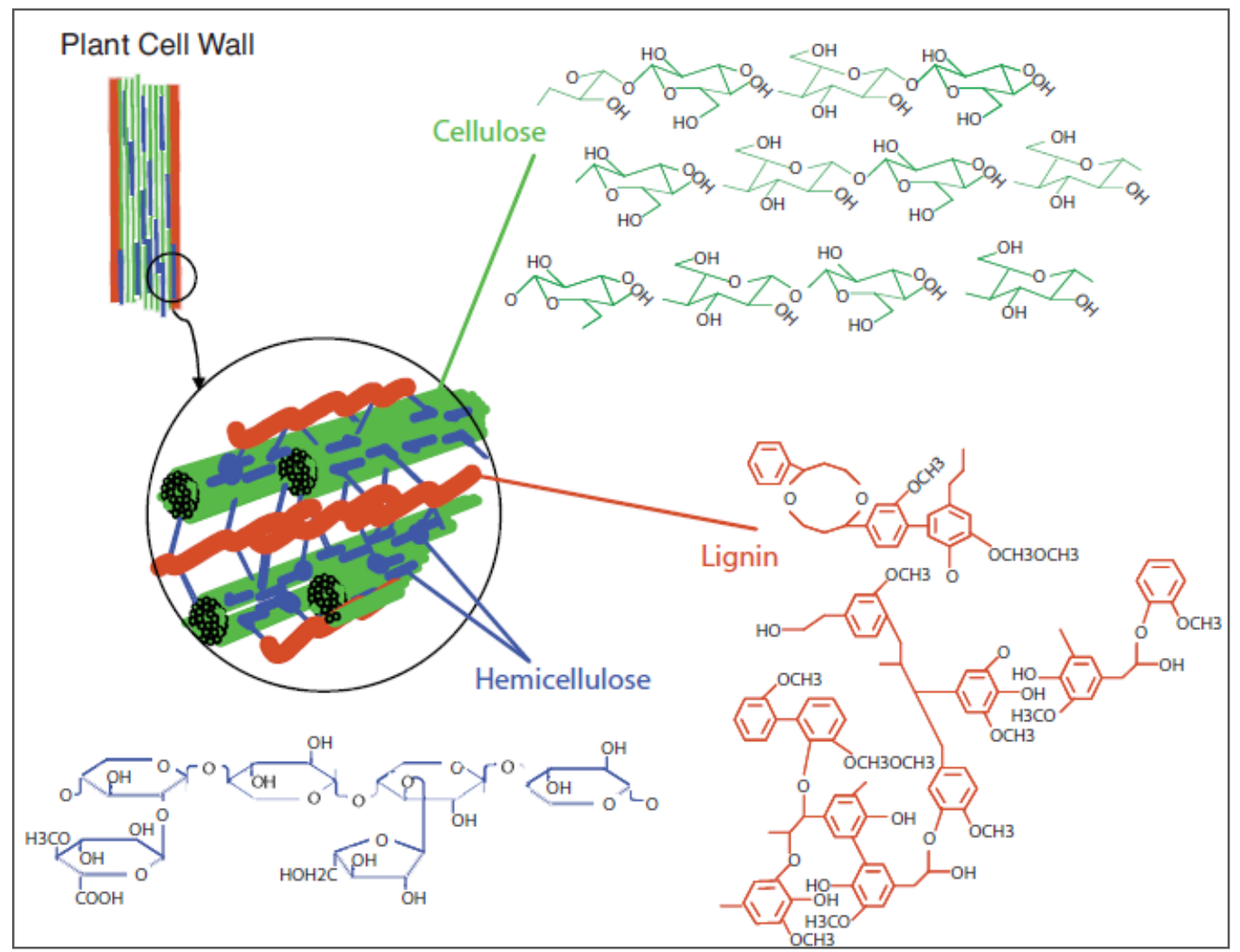

Figure 1.1: Lignocellulosic biomass constituents

Source: Genome Management Information System,Oak Ridge National Laboratory (2014)

Lignin is a very complex aromatic polymer composed of polyphenolic compounds arranged in branched chains. Lignin intermixes with cellulose and hemicellulose to form a matrix in the plant cell wall, providing strength to the plant and increasing its resistance to biodegradation (Wiselogel et al., 1996). Lignin from biomass can also be gasified to release the heat value contained to provide energy needed for conversion processes (Johansson et al., 1993).

Extensive research has been completed in the past on conversion of lignocellulosic biomass to ethanol (Dale et al., 1984; Cadoche and Lopez, 1989; Duff and Murray, 1996; Wright, 1998; Zhang and Lynd, 2010; Sathitsuksanoh et al., 2012; Bayens et al., 2014). As 
shown in Figure 1.2, processing lignocellulosic biomass to ethanol usually consists of four major unit operations: pretreatment, hydrolysis, fermentation, and product separation.

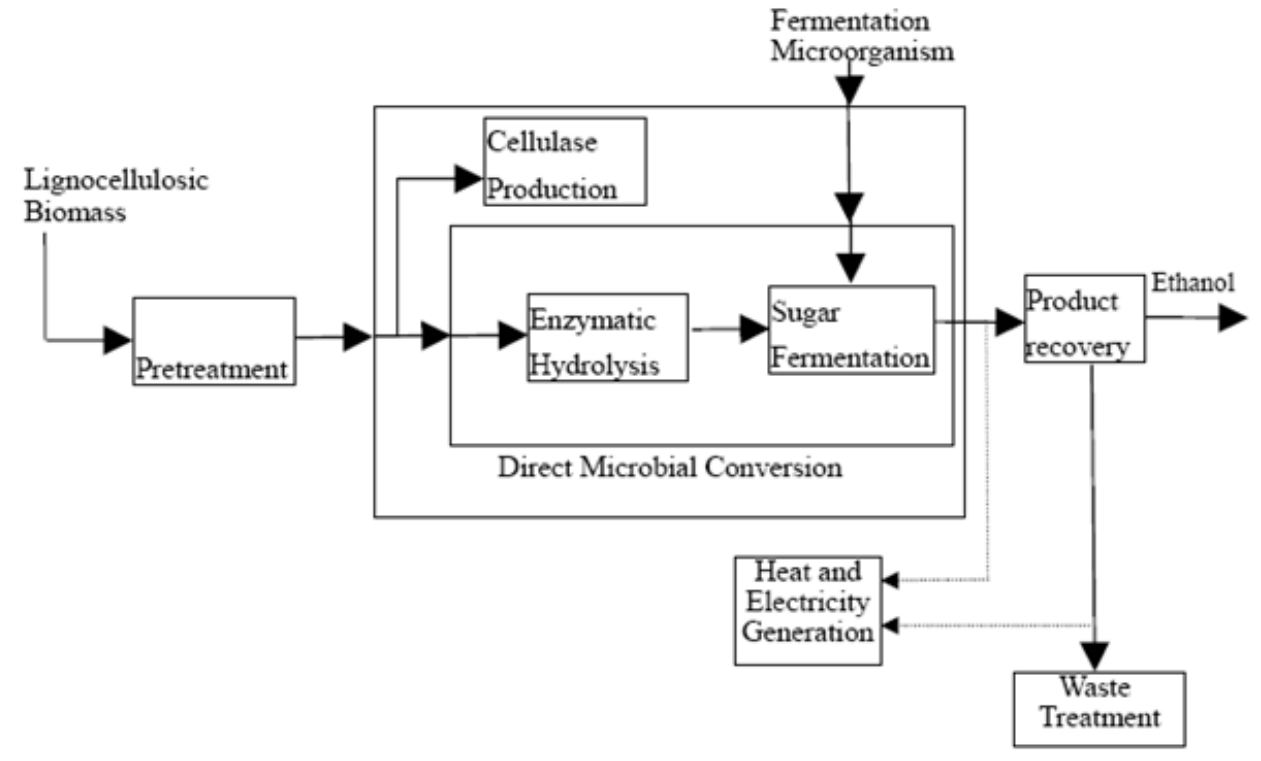

Figure 1.2: Major steps in conversion of lignocellulosic biomass to ethanol

The effect of pretreatment of lignocellulosic biomas has been recognized for a long time (McMillan, 1994). The purpose of pretreatment is to remove lignin and hemicellulose, reduce cellulose crystallinity, and increase the porosity of the material so that hydrolysis of carbohydrate fraction to monomeric sugars can be achieved more rapidly and with greater yields. Although a variety of process configurations have been studied for conversion of lignocellulosic biomass into an ethanol, enzymatic hydrolysis is deemed more competitive compared to other liquid fuels on a large scale (Wyman, 1999).

Cellulose can be hydrolytically broken down into glucose either enzymatically by cellulases, or chemically by sulfuric or phosphoric acids. Hemicellulases or acids hydrolyze the hemicellulose polymer to release its component sugars. Glucose, galactose, mannose, and six carbon sugars are readily fermented to ethanol by many naturally occuring organisms. On other 
hand, only very few native strains ferment five carbon sugars such as xylose and arabinose and usually at relatively low yields. Xylose and arabinose generally comprise a significant fraction of hardwoods, agricultural residues, and grasses. Table 1.1 summarizes the composition of most common lignocellulosic feedstocks. It maybe observed that typical carbohydrate contents range from 31\%- $81 \%$ by dry weight, and they stand from cellulose and hemicellulose.

Table 1.1: Percent dry weight composition of lignocellulosic feedstocks

\begin{tabular}{lccc}
\hline Feedstock & Carbohydrate & Carbohydrate & Non-carbohydrate \\
\hline & Glucan (cellulose) & Xylan (hemicellulose) & Lignin \\
& $\%$ & $\%$ & $\%$ \\
\hline Corn stover & 36.4 & 18.0 & 16.6 \\
Corn fiber & 14.28 & 16.8 & 8.4 \\
Pine wood & 46.4 & 8.8 & 29.4 \\
Popular & 49.9 & 17.4 & 18.1 \\
Wheat straw & 38.2 & 21.2 & 23.4 \\
Switch grass & 44.9 & 31.4 & 17.6 \\
Office paper & 68.6 & 12.4 & 11.3 \\
\hline
\end{tabular}

Source: Lynd et al., 1999

Cellulose and hemicellulose are potential sources of fermentable sugars (Ho et al., 1998; Taherzadeh et al., 1999; Sreenath and Jeffries, 2000). The presence of lignin in the cell wall, however, hamper enzymatic hydrolysis of the carbohydrates. The relationship between structural and compositional factors reflect the complexity of lignocellulosic materials. The variability in these characteristics accounts for the varying digestibility between different sources of 
lignocellulosic biomass. In general, effective pretreatment causes disruption of these barriers and prepares for enzymatic hydrolysis folowed by fermentation (Lynd et al., 1991; Holtzaplle, 1993; Mosier et al., 1999).

According to the cost analysis done by Lynd et al. (1999), among the various process steps, pretreatment and biological processing accounts for $32.7 \%$ and $39.6 \%$ respectively of total processing costs, which means that improvements are likely to reduce the the cost of ethanol production in both operational units to the level competitive with the cost of fossil fuels.

\subsection{Innovations in Biotechnology of Fuels and Cellulosic Feedstock Production}

Biotechnology of fuels is a versatile field with a goal to develop an alternative energy source. It stimulates research for alternative sources of energy via bio-chemical conversion processes. General movement is utilizing substantial amount of lignocellulosic biomass readily available for little cost. The earth has a huge stock biomass covering wide regions including forests and the ocean. The total biomass of the world is 1,800 billion tons on the ground and 4 billion tons in the ocean, and a comparative amount of biomass exists in the soil (The Asian Biomass Handbook, 2008). The total biomass on the ground is 33,000 EJ on the energy basis, which corresponds to 80 times or more of the annual energy consumption of the world. However, it is difficult to know the amount of waste biomass production in each country and region of the world. Therefore, the waste biomass production is often estimated typically by assuming ratio of waste production relative to the biomass resources production (The Asian Biomass Handbook, 2008). An example of the estimation of waste biomass production is shown in Table 1.2. 
Table 1.2: Estimation of waste biomass production and amount of resources

\begin{tabular}{lcc}
\hline Biomass species & $\begin{array}{c}\text { Ratio of waste production } \\
(\mathrm{t} / \mathrm{t})\end{array}$ & $\begin{array}{c}\text { Coefficient of energy } \\
\text { conversion }(\mathrm{GJ} / \mathrm{t})\end{array}$ \\
\hline Rice & 1.4 & 16.3 \\
Wheat & 1.3 & 17.5 \\
Maize (corn) & 1.0 & 17.7 \\
Roots and tubers & 0.4 & 6.0 \\
Sugarcane residue (tops & 0.28 & 17.33 \\
and leaves) & $1.10(\mathrm{t} / \mathrm{y} / \mathrm{head})$ & 15.0 \\
Cattle & $0.22(\mathrm{t} / \mathrm{y} / \mathrm{head})$ & 17.0 \\
Swine & 0.037 (t/y/head) & 13.5 \\
Poultry & $0.55(\mathrm{t} / \mathrm{y} / \mathrm{head})$ & 14.9 \\
Horses & $1.46(\mathrm{t} / \mathrm{y} / \mathrm{head})$ & 14.9 \\
Buffaloes and camels & $0.18(\mathrm{t} / \mathrm{y} / \mathrm{head})$ & 17.8 \\
Sheep and goats & 1.17 & 16.0 \\
Industrial logs & 0.67 & 16.0 \\
Fuel logs & 0.784 & 16.0 \\
Wood waste & & \\
\hline
\end{tabular}

Source: The Asian Biomass Handbook, 2008

The current stock of biomass is estimated based on waste biomass production multiplied by a coefficient of energy conversion. It is estimated at approximately $43 \mathrm{EJ}$ for livestock biomass, 48 EJ for agricultural biomass, and 37 EJ for forestry biomass, with totals of approximately 128 EJ. 
Approximately $22 \mathrm{EJ}$ of dung of cattle accounts for the largest part of resources, which is followed by an approximately $20 \mathrm{EJ}$ of log residue.

Conversion of lignocellulosic biomass to valuable biofuels has received the largest attention in past two decades and has concentrated on three main transformations: photosynthetic feedstock production; reduction of recalcitrance of biomass involving breakdown of complex components into simpler sugars; and end-product formation from sugars (Lynd, 2008). A number of studies indicate lignocellulosic feedstock could attain high yields (mainly in the form of ethanol) with positive balance in investment return (Lynd, 2008; Wyman, 2008). In Canada and US, for example, the goal is to provide more alternative greener fuels into the market and decrease the prevailing fossil fuel utilization. A conservative estimate places lignocellulosic crops at $\$ 50-\$ 60$ per metric ton (raw biomass), or converted to the price per energy value at $\$ 3 / G J$, which is equivalent to the value of crude oil at the $\$ 17$ per barrel (Lynd et al., 2008). There is a good potential for development of lignocellulosic fuels, but current implementation is still slow. This is because of the established corn ethanol alternative and due to retracted market for ethanol compared to gasoline (Bullis, 2013). However, there is a positive sign for a brighter future of lignocellulosic biomass, which eventually is expected to become a valuable product through government policy changes (Tyner, 2011) and improvements in new advances in the biotechnology realm.

\subsection{Source Separated Organic Waste as a Promising Cellulosic Feedstock}

While today ethanol is typically produced from the starch contained in grains such as corn, sugarcane, and grain sorghum, it also can be produced from cellulose which is mainly 
present in non-food products. Cellulose is the main component of plant cell walls and it is the most common organic compound on earth, which may be converted into usable sugars for ethanol production. Cellulosic ethanol is a blend of normal ethanol that can be produced from a great diversity of biomass including waste from urban, agricultural, and forestry sources.

Biomass such as processed source- separated organic (SSO) waste is particularly attractive in one context since it is widely available at negative cost and has many other benefits (e.g., good alternative fuel in terms of greenhouse gas (GHG) emissions, reduces of farm land's depletion, diminutive of generated waste) and life-cycle settings. Generally, SSO sample prepared for this study is a blend of approximately 78\%-80\% of organic green bin waste 20\%$22 \%$ of construction and demolition waste in form of wood chips, passed through the thermal screw press (TSP) (Vartek Waste Management Ltd, 2005). A type of the woodchips chosen may vary (Sims, 2004; EUBIA, 2007) and can be any kind of woody or agricultural waste as presented in Table 1.3, which may alter the composition.

The composition of biomass may vary depending on several local factors: 1) sorting criteria specified by the municipality for use by the households; 2) efficiency of the citizens in sorting properly; 3) collection system including the types of collection bags used in the kitchen (paper, plastic) and local storage bins (containers, paper sacks), in the so-called "green bin program”, and finally 4) pretreatment that is used (disc screen, screw separator, or magnetic separator) prior to the biological treatment (la Cour Jansen et al., 2004). 
Table 1.3: Classification of biomass resources

\begin{tabular}{|c|c|c|}
\hline Supply sector & Type & Example \\
\hline \multirow[t]{4}{*}{$\begin{array}{l}\text { Woody } \\
\text { Biomass }\end{array}$} & Dedicated forestry & $\begin{array}{l}\text { Short rotation plantations (e.g. willow, poplar, } \\
\text { eucalyptus) }\end{array}$ \\
\hline & Forestry by-products & Wood blocks, wood chips from thinning \\
\hline & Wood process residue & Bark, sawdust, shavings, wood chips and off-cuts \\
\hline & Recovered wood fuels & $\begin{array}{l}\text { Recovered wood fuels from activities such as } \\
\text { land clearance and municipal green waste }\end{array}$ \\
\hline \multirow[t]{5}{*}{ Agriculture } & $\begin{array}{l}\text { Dry lignocellulosic energy } \\
\text { crops }\end{array}$ & $\begin{array}{l}\text { Herbaceous crops (e.g. miscanthus, reed } \\
\text { canarygrass, giant reed) }\end{array}$ \\
\hline & $\begin{array}{l}\text { Energy crops } \\
\text { short rotation and annuals }\end{array}$ & $\begin{array}{l}\text { Oil seeds for methylesters (e.g. rape seed, } \\
\text { sunflower) } \\
\text { Sugar crops for ethanol (e.g. sugar cane, sweet } \\
\text { sorghum) } \\
\text { Starch crops for ethanol (e.g. maize, wheat) }\end{array}$ \\
\hline & $\begin{array}{l}\text { Agricultural residues } \\
\text { Livestock waste }\end{array}$ & $\begin{array}{l}\text { Straw, pruning from vineyards and fruit trees } \\
\text { Wet and dry manure (cattle, pigs, horses and } \\
\text { poultry as well as human ) }\end{array}$ \\
\hline & $\begin{array}{l}\text { Agro-industrial by- } \\
\text { products }\end{array}$ & Bagasse, rice husks \\
\hline & Water vegetation & Algae, water hyacinths, seaweeds \\
\hline Industry & Industrial residue & $\begin{array}{l}\text { Industrial waste wood, sawdust from sawmills } \\
\text { Fibrous vegetable waste from paper industries }\end{array}$ \\
\hline \multirow[t]{2}{*}{ Waste } & Dry lignocellulosic & $\begin{array}{l}\text { Residues from parks and gardens (e.g. prunings, } \\
\text { grass) }\end{array}$ \\
\hline & Contaminated waste & $\begin{array}{l}\text { Demolition wood } \\
\text { Organic fraction of municipal solid waste } \\
\text { Biodegradable landfilled waste, landfill gas } \\
\text { Sewage sludge }\end{array}$ \\
\hline
\end{tabular}

Source: Sims, 2004; EUBIA, 2007 


\subsection{Ethanol Prediction Yields}

Four approaches for biomass process configuration featuring enzymatic hydrolysis and fermentation have been reported in the literature (Lynd et al., 2002): separate hydrolysis and fermentation (SHF), simultaneous saccharification and fermentation (SSF), simultaneous saccharification and co-fermentation (SSCF), and consolidated bio-processing (CBP).

Depending on the type of biomass/feedstock and process configuration (SHF, SSF, SSCF, CBP), actual yield of the ethanol could be anywhere from $60 \%$ to $90 \%$ of theoretical (Dowe and McMillan, 2008). According to Dowe (2009) the stoichiometric maximum theoretical yield of ethanol is concluded at $0.51 \mathrm{~g}$ ethanol / g glucose or xylose. Theoretical yield of greater than $90 \%$ from glucose is achievable by robust fermentative organisms such as Saccharomyces cerevisiae and Zymomonas mobilis (Chu and Lee, 2007).

Currently estimated yields from cellulosic biomass are expected to increase as conversion technologies mature with greater flexebility to accommodate differenet feedstock compositions. It is clear that lignocellulosic biomass offers an opportunity for energy production but there are many social, political, economic and environmental conditions that affect the scale of this production. Since none of these conditions are static, there is unlikely to be a definitive calculation for the amount that can be produced. In the rough estimation, the international consensus summarized in the IPCC Special Report on Renewable Energy sources and Climate Change Mitigation (SRREN) that 100EJ - 300EJ per year can be achieved from biomass by 2050 (Davis et al., 2014). 


\subsection{Selection of Microorganisms}

Microorganisms for ethanol fermentation are typically evaluated in terms of the following performance parameters: ethanol yield, inhibitor tolerance, substrate productivity, growth rate, $\mathrm{pH}$, and temperature range. Currently, attempts to develop microorganisms are primarily focused in two areas: a) developing universal microorganisms that can utilize various sugars; and b) developing microorganisms for the specific processing configuration (SHF, SSF , SSCF, CBP). Generally, there are two strategies for developing microorganisms to enhance the range of sugars used. The first strategy considers microorganisms that can utilize different substrates. It is called the "recombinant substrate utilization strategy". The second strategy is the so-called "native substrate technology”, which implies microorganisms that naturally use multiple sugars and enhance their ability to produce ethanol (Lynd et al., 1999).

A number of different microorganisms have been employed in the fermentation of the water-soluble lignocellulose derived hexose sugars to ethanol. For the most part, the yeast Saccharomyces cerevisiae has been used for fermentation.

To overcome the concerns with pentose sugars fermentation, some researchers have explored the use of alternative microorganisms such as Pichia stipitis, Pachysolen tannphilus, and Candida shehatae (Jeffries, 1983; Hahn-Hagerdal et al., 1993). These microorganisms can ferment xylose to ethanol, but have low productivity and ethanol yield (du Preez et al., 1989; Hahn-Hagerdal et al., 1994a) and increased nutritional and aeration requirements (Dellweg et al., 1984; du Preez et al., 1985; Skoog and Hahn-Hagerdal, 1990). These microorganisms may also show increased sensitivity to ethanol, resulting in inhibition of both growth and fermentation (du Preez et al., 1989). Moreover, they do not metabolize the other sugars such as glucose as fast as S.cerevisiae (du Preez et al., 1986; Hahn-Hagerdal et al., 1994b) and maybe more vulnerable to 
potential inhibitors presented in water soluble fraction (Linden and Hahn-Hagerdal, 1989;

Delgenes et al., 1996).

A series of recombinant $S$. cerevisiae strains that can utilize xylose have been developed in past decades at Purdue University (Moniruzzaman et al., 1997; Ho et al., 1998). Strains of $S$. cerevisiae are capable of high rates of ethanol production from a range of carbon sources, displaying high ethanol tolerance (Ingram, 1986), and increased resistance to potential inhibitor compounds (Martin and Jonsson, 2003). However, a main obstacle of bioconversion with earlier S. cerevisiae strains were an inability of microorganisms to metabolize pentose sugars (xylose and arabinose) into ethanol, especially from harwood and agricultural residues. Agricultural residues and hardwoods are similar in the way that they have lower lignin content and the hemicellulose produces significant amounts of pentose sugars. On the other hand, softwoods have a higher lignin content, which makes the hydrolysis step more difficult, but they generally produce less pentose sugars.

More recent development in gene engineering has resulted inrelatively new recombinant microorganisms such as S. cerevisiae RWB222 and S. cerevisiae DA2416 avilable for biomass conversion with ability to utilize pentose sugars. S. cerevisiae RWB222 strain was genetically modified derivative of $S$. cerevisiae CEN, PK. Xylose utilization in this strain was achieved by integration of the xylose isomerase from Piromyces sp. E2, over expression of the native pentose phosphate pathway, and directed evolution for growth on xylose (van Dijken et al., 2000; Kyper et al., 2005). The isomerase is vital for S. cerevisiae to grow well on xylose under anaerobic conditions with high ethanol production (Maris et al., 2006).

In another study, Suk-Jin Ha et al. (2011) have developed a unique strategy to coferment a mixture of xylose and cellobiose with S. cerevisiae DA2416 strain (derivative of S. cerevisiae 
D452-2). They introduced a newly discovered cellodixtrin transporter and intracellular $\beta$ glucosidasefrom cellulotic fungi, Neurospora crassa (Galazka et al., 2010) into a S. cerevisiae DA2416 strain. It was engineered to ferment xylose with improved ethanol yields of $0.39 \mathrm{~g} / \mathrm{g}$ from a mixture of cellobiose and xylose as compare to ethanol yields of $0.31-0.33 \mathrm{~g} / \mathrm{g}$ from fermentation either cellobiose or xylose as sole carbon sources. These results suggest that cofermentation of glucose and xylose can enhance the overall ethanol yields and productivities.

Along with the development of S. cerevisiae strains, a series of bacteria Zymomonas mobilis that can use xylose and/or arabinose were developed in the National Renewable Energy Laboratory (Zhang et al., 1995; Deanda et al., 1996; Zhang et al., 1998). They illustrated the recombinant substrate utilization strategy. Z. mobilis is the native ethanogenic bacterium with a high ethanol yield. This microorganism can produce greater quantities of ethanol per mole of biomass produced due to the Enther-Doudoroff metabolic pathway. However, the natural substrate range of $Z$. mobilis is restricted to the fermentation of glucose, fructose, and sucrose, limiting its applicability for biomass tranformation (Lindsay et al., 1995).

To overcome these shortages, engineered Z. mobilis strains were developed (Zhang et al., 1995). These engineered microorganisms were likely intended to be superior in lignocellulosic bioconversion, but commercial application did not take place. Among the further achievements were two engineered Z. mobilis strains: Z. mobilis 8b (Joachimsthal et al., 1999) and Z. mobilis AX101 (Kompala et al., 2001). Z. mobilis 8b was developed as an integrant of Z. mobilis ZM4 tolerant up to $16 \mathrm{~g} / \mathrm{L}$ acetic acid and it can grow from $\mathrm{pH} 3.5 \mathrm{up}$ to $\mathrm{pH}$ of 7.0 with optimum $\mathrm{pH}$ value range from 5.0 to 6.0 in ethanol production process (Kim et al., 2000). Its rival, Z. mobilis AX101, is capable of fermenting both xylose and arabinose effectively, with the yield achiviement of more than $80 \%$ of theoretical within 50 hours (Kompala et al., 2001). 
In a new study, Mohagheghi et al. (2015) have adapted and evolved Z. mobilis strain 8b for enhanced tolerance to the toxic inhibitors present in corn stover hydrolysate. The adapted strain, named SS3, has a higher xylose utilization rate and produces more ethanol than the parent strain, providing foundation for future research directions in improving Z. mobilis for ethanol production and other fuel precursors.

Based on todays' market availability and their ability to utilize glucose and xylose sugars from pretreated biomass in the fermentation phase, robustness and tolerance to inhibitors, two different recombinant glucose and xylose utilizing strains, Z. mobilis 8b and S. cerevisiae DA2416, were chosen for detailed study in the investigation of SSO waste for ethanol production.

\subsection{Ethanol production and inhibitors}

In the study of ethanol production, many researches (Philippidis et al., 1992; McMillan et al., 1999; Lynd et al., 2001; Kumar et al., 2009; Zhang et al., 2009) have identified and measured certain parameters: maximum specific rates, ethanol inhibitors and yield, $\mathrm{pH}$, and temperatures. In most cases of measuring maximum specific rates, the initial substrate concentartion is kept low (below $10 \mathrm{~g} / \mathrm{L}$ ), so the effect of produced ethanol could be ignored.

The effect of ethanol on maximum specific rate, $\mu_{\mathrm{m}}^{\mathrm{o}}$, is usually described by the noncompetitive inhibition equation by van Uden (1989) as in the following:

$$
\mu=\frac{\mu_{m}^{o} \cdot S}{K_{S}+S} \cdot \zeta(\rho)
$$


where $\mu$ - measured specific growth rate under different ethanol concentartions; $\mu_{m}^{o}$ - maximum specific growth rate with no initial ethanol; S-substrate concentration; $\mathrm{K}_{\mathrm{s}}$ - Monod saturation constant; $\zeta(\rho)$ - ethanol inhibition function.

The first multiplier of the above equation is simply the Monod equation frequently used in simulating the exponential, retardation, and stationary phases of butch culture as well as chemostat steady state. It applies best to media processing a single growth limiting nutrient, an essential nutrient which runs out completely and stops growth before the concentration of any other nutrient has been reduced to a level which will affect the kinetics. During the course of fermentation process, ethanol accumulates in the broth to such extent that metabolic activities of microorganisms is supressed. Therefore, the presence of ethanol decreases the value of specific growth rate and equation above must be extended to include ethanol inhibition function, $\zeta(\rho)$.

The ethanol inhibition effect typically exhibit different patterns: a) linear kinetics; b) linear kinetics with threshold concentration; c) nonlinear concave up kinetics; and d) nonlinear concave down kinetics (van Uden, 1989).

Several nonlinear ethanol inhibition were found and presented below in the form of the equations by different researchers (Dean, 1964; Aiba et al., 1968; Bazua, 1977; van Uden, 1989) to describe the nonlinear ethanol inhibition:

$$
\zeta(\rho)=\frac{K}{K_{s}+P}
$$

van Uden (1989) has proposed expression for $\zeta(\rho)$ in equation above, which is identical with those derivable for noncompetitive inhibition using the Michaelis-Menten equilibrium approach to enzyme kinetics. Such expression, however lack accurate basis when applied to the growth of whole cells. 
Equation (3) and Equation (4) below proposed (Aiba et al., 1968; Bazua, 1977)

respectively are exponential models from experiments on alcohol fermentation with respirationdeficient mutant of $S$. cerevisiae, where the empirical constant $K_{p}$ appears to be depend on the method of cultivation (batch or continuous):

$$
\begin{gathered}
\zeta(\rho)=e^{-K_{\rho} \cdot P} \\
\zeta(\rho)=1-\frac{P}{P_{m}}
\end{gathered}
$$

Equation (5) below was suggested (Dean, 1964) to describe the kinetic pattern of product inhibition of the strain $S$. cerevisiae, since there was a nonlinear relation (concavity downward) between $\mu_{i}$ and P. Equation (5) accounts for the influence of ethanol product, however, resulting values were quite different from experimental data.

$$
\zeta(\rho)=\left(1-\frac{P}{P_{m}}\right)^{f_{1}}
$$

where P - added ethanol concentration; $K_{P}, P_{m}$, and $f \mathbf{1}$ - ethanol related constants.

There are several other important parameters that have been identified in the studies for ethanol production from lignocellulosic biomass. Among them arethe net rate of glucose formation and glucose fermentation, respectively by Zhang et al. (2009) as shown in equations below:

$$
r_{G l}=\frac{d G l}{d t}=\left[\frac{K_{C} \cdot c_{b} \cdot B G}{K_{m} \cdot\left(1+\frac{G l}{K_{C g}}\right)+c_{b}}\right] \cdot 1.053-\left(\frac{\mu_{G l}}{Y_{X / G l}^{\max }}+m_{G l}\right) \cdot X
$$




$$
\mu_{G l}=\left[\frac{X \cdot \mu_{G l}^{M a x} \cdot G_{l}}{K_{G l}+G_{l}+I_{1} \cdot X_{l}}\right] \times\left(1-\frac{E t h}{E t h_{G l}^{M a x}}\right)^{f 1}
$$

where $\mu_{G l}^{M a x}$ - maximum specific rate for growth on glucose; $E t h_{G l}^{M a x}$ - maximum ethanol concentration for growth on glucose; $G_{l}, X_{l}$, and Eth - concentrations of glucose, xylose and ethanol respectively; $C_{b}, B G$, and $\mathrm{X}$ - concentrations of cellobiose, $\beta$-glucosidase and cell mass; $\mu_{G l}, Y_{X / G l}^{\max }, m_{G l}$ - specific growth rate, maximum cell mass yield on glucose andmaintenance coefficient on glucose;1.053 - coefficient for water added during cellulose hydrolysis; $K_{c}, K_{m}, K_{C g}$ - cellobiose hydrolysis related constants; $K_{G l}, I_{1}, f_{1}$ - related constants. The rate of formation of xylose was described by an approach similar to that used for glucose and could be found elsewhere (Zhang et al., 2009) as in the following equations:

$$
\begin{aligned}
& r_{X l}=\frac{d X l}{d t}=-r_{X n} \times 1.136-\left(\frac{\mu_{X l}}{Y_{X / X l}^{M a x}}+m_{X l}\right) \times X \\
& \mu_{X l}=\left[\frac{X \times \mu_{X l}^{M a x} \times(X l-X l T)}{\left(K_{X l} \times X+X l+I_{2} \times G l\right)}\right] \times\left(1-\frac{E t h}{E t h_{X I}^{M a x}}\right)
\end{aligned}
$$

where $\mu_{X l}^{M a x}, E t h_{X l}^{M a x}$ - maximum specific growth rate and maximum ethanol concentration for growth on xylose, respectively; $I_{2}, K_{X l}$ - related constants; XlT-threshold concentration, which is related to maintenance coefficient $m_{X l} ; Y_{X / X l}^{\operatorname{Max}}, \mu_{X l}^{\operatorname{Max}}$ - maximum cell mass yield and maximum specific growth rate; $K_{X l}$ - Monod saturation constant; 1.136 - coefficient for water added during xylan hydrolysis.

It is been reported (Zhang et al., 2009) that among the microbial growth related constants the maximum specific growth rate on xylose, $\mu_{X l}^{\max }$ showed moderate sensitivity, while the 
maximum specific growth rate on glucose, $\mu_{\mathrm{Gl}}^{\max }$ showed no sensitivity at all even with a $50 \%$ change of the value. Meantime, the ethanol yield from glucose $\mathrm{Y}_{\mathrm{Eth} / \mathrm{GL}}$ exhibits the highest sensitivity, followed by ethanol yield from xylose $\mathrm{Y}_{\mathrm{Eth} / \mathrm{Xl}}$. Ethanol tolerance related constants $E t h_{G l}^{\max }, E t h_{X l}^{\max }, \zeta l$ showed moderate sensitivity. Also, sugar inhibition factors $I_{1}$ and $I_{2}$ and the Monod constants $\mathrm{K}_{\mathrm{Xl}}$ and $\mathrm{K}_{\mathrm{Gl}}$ showed a low sensitivity during the SSCF experimental testing.

At the present time, several biological methods can be used to overcome inhibitory effects of aliphatic acids, furaldehydes or phenolic compounds on yeast fermentation in pretreated lignocellulosic materials. Possible options include detoxification of the pretreated material before fermentation by using available enzyme complexes to mediate enzymatic hydrlosis or use of the natural or targeted genetic engineered bio-reduction capability of the fermenting micro-organism which will detoxify the medium during the fermentation (Parawira and Tekere, 2011). The ability to degrade inhibitors exists in S. cerevisiae and other microorganisms and we only need to exploit or enhance this natural strategy to overcome inhibitors in lignocellulose biomass in some cases through adaptation and genetic engineering. The fermentation can be carried out in a process design such as batch or continues fed-batch that will allow for the natural reduction capability of the micro-organisms to be exploited.

Adaptation (evolutionary engineering) of the fermentation micro-organisms to the lignocellulosic hydrolysate has been also suggested as an alternative detoxification approach (Martin et al., 2007). Although chemical, enzymatic, and microbial detoxification improves the fermentability of hydrolysates, it is desirable to develop adaptive ethanol-producing microorganisms that require minimal or no detoxification treatment. These adapted organisms not only reduce the detoxification cost, but also avoid loss of fermentable sugars (Martín et al., 2007). Adaptation has been shown to increase the ability of a broad range of yeast strains to grow in 
lignocellulosic hydrolysates, resulting in increased fermentation rates and ethanol yields (Parawira and Tekere, 2011). Therefore to conclude, removal of inhibitors is necessary for achieving good fermentation performance even with newly recombinant glucose-xylose utilizing yeasts.

\subsection{Process Governing Factors}

Evidently, inhibitors play a significant role during the fermentation of the sugars in water-soluble fraction to ethanol. Usually they are composed in one of two categories: process derived inhibitors arising from pretreatment (sugar, lignin from degradation products) or naturally occuring inhibitors liberating from the feedstock and recovered in the water soluble fraction (resin, acids). Inhibitors in both categories play a significant role in the fermentation process. Besides sugar decomposition, lignin is the greatest concern of the process derived inhibitors. Lignin can degrade under certain acidic conditions, primarily by the cleavage of the aryl ether bonds at the $\alpha$ - and $\beta$ - positions (Lai, 1991). Limited solubilization of lignin via sulphonation has been also reported (Clark et al., 1989) and made this inhibitor an indispensable contributer to inhibiton. The lignin derived phenolics have been shown to inhibit lignocellulosic fermentation, both for the production of ethanol by S. cerevisiae and 2,3-butanediol by Klebsiella pneumoniae (Nishikawa et al., 1988). Inhibition is related to the disruption of the plasma membrane as well as to the molecular weight of the compound, which affects its permeability (Ando et al., 1986). Removal of these inhibitors could be achieved by extraction with solvents to improve subsequent fermentation step (Clark and Mackie, 1984; Frazer and McCaskey, 1989).

As mentioned early, the naturally occuring inhibitors are mainly of extractives origin. Biologically, many of these extractive components play a defensive role against microbes and 
insects in protecting the wood from decay (Haygreen and Bowyer, 1996), and as a result, it’s expected to be harmful to fermentation efficiency. However, as for lignin derived phenolics, the concentration of these compounds recovered in the water soluble fraction is often low due to its limited solubility and may not be exceedingly aleatory to subsequent fermentation process.

Among the other important factors that play a considerable role in the fermentation process are $\mathrm{pH}$ value and temperature. These factors are well documented and understood in the literature (Lawford et al., 1997; Moniruzzaman et al., 1997; Joachimsthal et al., 1998; Lawford and Rousseau, 1998; Mohagheghi et al., 1998; Teixeira et al., 2000; Mohagheghi et al., 2004; Kim et al., 2008; Zhang, 2008; Lin et al., 2012; Tsuji et al., 2013).

Study of Kim et al. (2008) identified a direct correlation of ethanol production and other factors including $\mathrm{pH}$ and temperature in food waste. In their work, response surface methodology (RSM) based on the central composite design (CCD) was used for the optimization of enzymatic saccharification and ethanol production. A combination of factors generating a certain optimum response can be identified. Optimal conditions, particurarly for fermentation, were reported as pH 6.85 and temperature of $35^{\circ} \mathrm{C}$. Ethanol yield was obtained as $57.5 \mathrm{~g} / \mathrm{L}$ under these conditions with a fermentation time of 14 hours. Experimental results were in close agreement with the model prediction and statistical validity. Other comparison study (Zhang, 2008) reported a good performance of strain Z. mobilis 8b over strain S. cerevisiae RWB222 for SSCF configuration in paper sludge experiments yielding more than $0.38 \mathrm{~g} / \mathrm{L}$ of ethanol under anaerobic conditions. Table 1.4 outlines some data of this study. 
Table 1.4: Selection of strains for paper sludge SSCF

\begin{tabular}{|c|c|c|c|c|}
\hline Microorganism & $\begin{array}{c}\text { Ethanol } \\
\text { Tolerance } \\
\text { desired }\end{array}$ & $\begin{array}{l}\text { Good performance } \\
\text { near pH } 5.5\end{array}$ & $\begin{array}{l}\text { Good ethanol yield } \\
\text { under anaerobic } \\
\text { condition }\end{array}$ & $\begin{array}{l}\text { Yield higher than } \\
0.38 \text { with } \\
\text { combination of } \\
\text { glucose/xylose = } 4 \\
\text { under anaerobic } \\
\text { condition }\end{array}$ \\
\hline E. coli K011 & No & No & Yes & Yes \\
\hline E.coli FBR5 & No & No & Yes & Yes \\
\hline K. oxytoca P2 & No & N/A & Yes & Yes \\
\hline Z. mobilis $8 b$ & Yes & Yes & Yes & Yes \\
\hline $\begin{array}{l}\text { C. shehatae CSIR- } \\
\text { Y492 }\end{array}$ & No & No & No & N/A \\
\hline $\begin{array}{l}\text { P. stipitis CSIR- } \\
\text { Y633 }\end{array}$ & N/A & Yes & No & No \\
\hline $\begin{array}{l}\text { Saccharomyces sp. } \\
1400\end{array}$ & Yes & Yes & No & Yes \\
\hline $\begin{array}{l}\text { S. cerevisiae } \\
\text { RWB222 }\end{array}$ & Yes & Yes & Yes & Yes \\
\hline
\end{tabular}

Source: Zhang, 2008

Results showed paper sludge SSCF by Z. mobilis $8 \mathrm{~b}$ had a much higher ethanol yield at $30^{\circ} \mathrm{C}$ than $37^{\circ} \mathrm{C}$ to the better consumption of residual sugar, and higher final glucan and xylan conversion. Yet, paper sludge SSCF by S. cerevisiae RWB222 had a higher ethanol yield at $37^{\circ} \mathrm{C}$ than $30^{\circ} \mathrm{C}$, apparently due to enzymatic activity at a higher temperature. It was also concluded that the best ethanol productivity could be achieved at the temperature between $30^{\circ} \mathrm{C}$ and $37^{\circ} \mathrm{C}$ with a pH value between 5.0 and 6.0, which support good growth and utilization ability of Z. mobilis 8b strain. 
The influence factors such as temperature, substrate concentration and $\mathrm{pH}$ affecting ethanol fermentation using S. cerevisiae BY4742 was demonstrated in Lin et al. (2012) study. Fermentation of sugar by S. cerevisiae BY4742 for production of ethanol in a batch experiments was conducted to improve the performance of the fermentation process. Experimental results revealed that the cellsmass increased exponentially at the beginning of incubation,then entered a stationary phase after several days incubation, for different operating temperatures from $20^{\circ} \mathrm{C}$ to $50^{\circ} \mathrm{C}$. Higher temperatures made the exponential growth of the cellsmass shorter. In their study, cell growth and ethanol production declined considerably at $50^{\circ} \mathrm{C}$, which showed the inhibition effect on cell growth at higher temperatures. They explained that the high temperature results in changing the transport activity or saturation level of soluble compounds and solvents in the cells, which might increase the accumulation of toxins including ethanol inside the cells.

In the case of substrate concentrations, they found that higher substrate concentration may achieve higher ethanol production, but a longer incubation time was required for higher initial glucose concentration above $80 \mathrm{~kg} / \mathrm{m}^{3}$ at a temperature of $30^{\circ} \mathrm{C}$. However, with glucose concentration of $300 \mathrm{~kg} / \mathrm{m}^{3}$, the ethanol conversion efficiency is decreased considerably (13.7\% versus $59.9 \%$ for $80 \mathrm{~kg} / \mathrm{m}^{3}$ ), since the higher substrate and production concentrations may have inhibited the process of ethanol fermentation.

In addition to temperature and substrate concentration, $\mathrm{pH}$ is also a key factor that affects on fermentation process for ethanol production. Lin et al. (2012) found that the $\mathrm{pH}$ range of 4.0 5.0 maybe regarded as the operational limit for the anaerobic ethanol production process. Beyond this range, the formation of by-products, such as acetic and butyric acids may have consumed some of the substrate and reduced the efficiency of fermentation process. Therefore, a robust tolerant yeast is desired to maintain a high ethanol yield with increased substrate loadings 
in fermentation phase. Eventually, it will lead to reduction of the amount of enzymes used in pretreatment and enzymatic hydrosis phase, often accounting for up to $40 \%$ of the total processing cost (Zhu et al., 2009). Moreover, it greatly affects the downstream cost of fermentation. 


\section{CHAPTER 2. THESIS OBJECTIVES AND AUTHORSHIP}

\subsection{Thesis Objectives}

Since the middle of the 70's, significant research and development has brought about numerous research groups for the improvement of lignocellulosic bioconversion to ethanol. However, currently, there is still limited commercialization of a process that can produce the "potential transportation fuel of the future” (Lynd et al., 1996). Most agreed that the present state economy of lignocellulosic ethanol still does not allow commercial production (Banerjee et al., 2010; Zhoa et al., 2012; Baeyens et al., 2015). This is further complicated by the global fluctuations of gasoline prices in recent year. Bioconverison of lignocellulosic biomass into ethanol and other energy value products is regarded as a very complicated process. Nevertheless, clean, renewable energy is always a worthwhile pursuit. There is a great need of continued breakthroughs in advanced technology to achieve this goal.

The current project was initiated at Ryerson University, Toronto, Canadato confirm that various sources of biomass from construction/demolition, organic "green bin" wastes, agricultural and forestry residues can be pre-processed to change the characteristics of biomass. Thereafter, the pretreated biomass can be fermented into alcohols and converted into other useful products such as ethanol, chemicals and gases. This study will lead to the development of an efficient method utilizing non-food-based biomass (e.g. municipal solid waste) to produce ethanol in much more sustainable ways than current practices, which utilize food-based biomass such as corn, sugarcane and wheat more commonly. 
The main objective of this research is to investigate the bioconversion process of pretreated source separated organic (SSO) waste by separate hydrolysis and fermentation (SHF) processing approach for ethanol production. The solution is to use "pre-processing" technologies, including the thermal screw press (TSP), cellulose and organic-solvent (ethanol/aceton) based lignocellulosic fractionation (COSLIF) preatretment to fractionate lignocellulosic biomass and prepare it for further fermentation process with bacteria or yeast. Lignocellulosic biomass such as pre-processed SSO waste is particularly attractive since it is widely available, often at negative or low cost and has a great potential for bioconversion in ethanol production.

Specific objectives of the study are: a) verify pretreatment technologies, such as thermo screw press and COSLIF; b) evaluate the performance of the COSLIF pretreatment on SSO feedstock for ethanol production; c) compare the growth and fermentation performances of pretreated SSO waste on ethanol production of two glucose/xylose utilizing strains: Zymomonas mobilis 8b and Saccharamyces cerevisiae DA2416; and d) interpolate new data of SSO feedstock into an existing kinetic model capable in predicting its behaviour under specified conditions. Figure 2.1 below shows the structure of this thesis which is made up from 3 journal papers published to achieve the specific objectives.

The thermal screw press (TSP) machine is chosen to be used in this study for processing biomass in a new unique way such that the feedstock is exposed to crushing, mixing, homogenizing, granulating, cell decomposition, compacting, heat generating and moisture reduction, all in one step. Compressed products such as peat log, wood chips/briquettes, fertilizer sticks can be extruded and formed into various shapes based on die design. The machine is able to densify on a continuous basis and extrude product in most cases without the need of binders. 


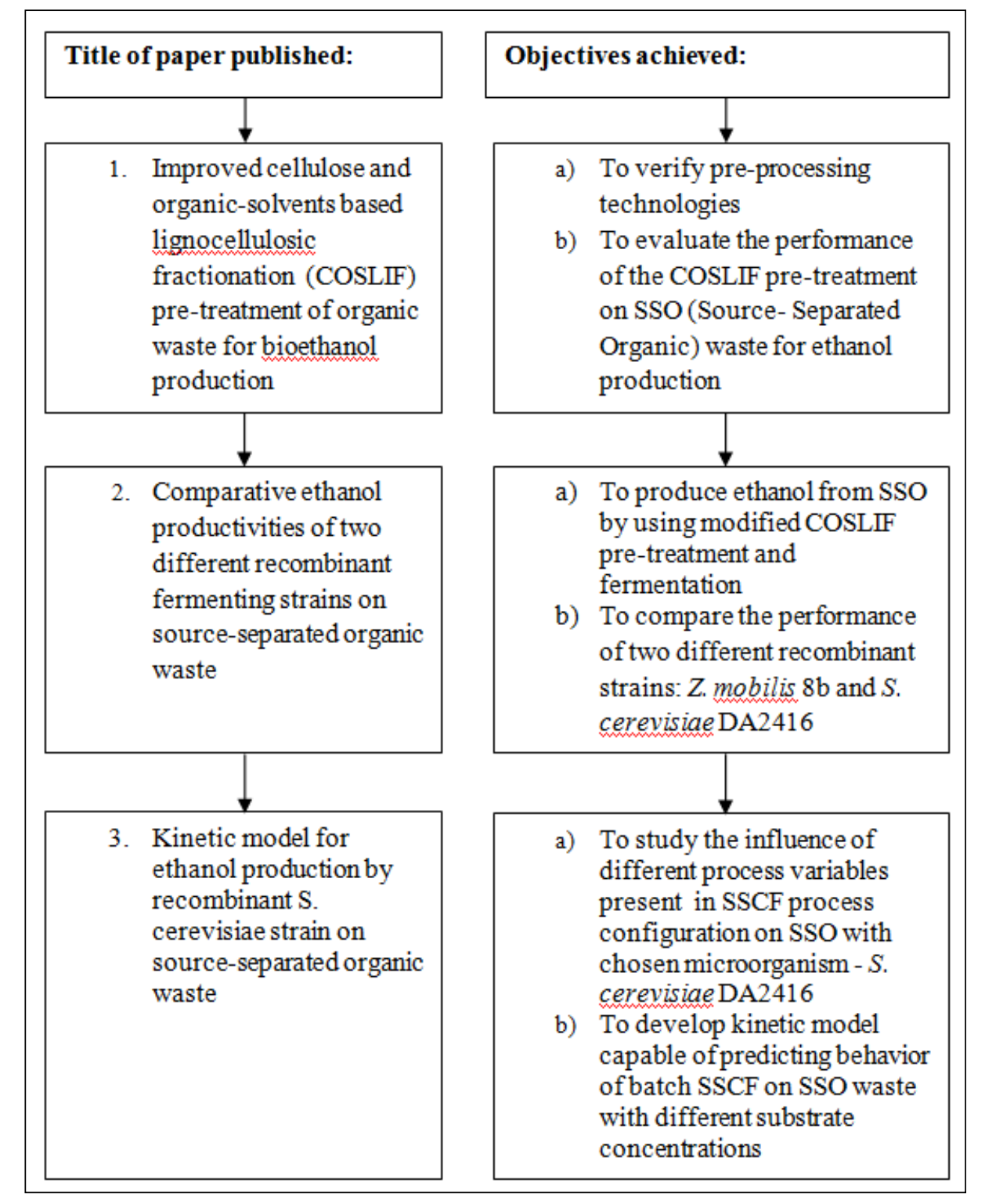

Figure 2.1: Structure and objectives of the study

Among different types of pretreatment technologies, COSLIF pretreatment is adapted in this work due to its impressive glucose yield (approximately 90\%), obtained from our preliminary testing (Bekmuradov et al., 2014). COSLIF pretreated SSO substrate will be verified with those 
from similar studies and will be further validated with performance of commercially available enzyme complex - Accellerace 1500 in the enzymatic hydrolysis phase.

This study utilized pretreated SSO waste as a model substrate to investigate the SHF configuration with fermentation by two recombinant xylose utilizing strains: Zymomonas mobilis 8b and Saccharomyces cerevisiae DA2416, which are prominent recombinant strains that utilize xylose and produce ethanol at high yields. The above mentioned strains were chosen for a detailed study of SHF process configuration due to their robustness, ethanol yield, and ethanol tolerance relative to other xylose utilizing recombinant organisms that were tested previously under similar conditions (Zhang, 2008).

This project is a first and potentially the only one of its kind to include process investigation, verification and mathematical modeling on SSO wasteas a feedstock for ethanol production from start to finish. Fermentation and hydrolysis kinetic parameters will be defined experimentally in this study.

Major contributions to the scientific community from this study will include: the understanding of general waste composition from municipal waste streams; cellulose and sugar contents in SSO samples; pretreatment methods that work best for SSO feedstock; comparison of performances on ethanol yields by yeast versus bacterium; and elicitation of significant factors for process control and optimization in the bioconversion process.

It is very timely research with useful application and implications not only in waste management and environmental pollution control, but also in energy sector, food crop, and economics. 


\subsection{Thesis Outline and Statement of Authorship}

\subsubsection{Thesis Outline}

This thesis is composed of 6 chapters. Chapters 1 and 2 present the introduction and literature review, as well as the main goals for this thesis. Chapters 3 to 5 comprise of three Journal papers describing the main findings of the research program. The performance investigation of COSLIF pretreated SSO is presented in Chapter 3. It describes an improvement on the standard method of COSLIF pretreatment based on lower enzyme loading and using an ethanol washing instead of acetone. Chapter 4 contains a comparison of the growth and fermentation performances of pretreated SSO waste on ethanol productivities of two glucose/xylose utilizing recombinant strains: Zymomonas mobilis 8b and Saccharomyces cerevisiae DA2416. Chapter 5 describes the mathematical kinetic modeling to accommodate batch simultaneous saccharification and co-fermentation process of the SSO waste by the recombinant strain, Saccharomyces cerevisiae DA2416. It includes the model calibration, estimation of parameters, and sensitivity analysis of an existing semi-mechanistic kinetic model as applied to SSO. Chapter 6 presents conclusions and recommendations of future work.

\section{Chapter 1: Introduction}

Chapter 2: Thesis Objectives and Authorship

Chapter 3: Improved Cellulose and Organic-Solvent- based Lignocellulosic Fractionation Pretreatment of Organic Waste for Bioethanol Production

This chapter has been published in the American Journal of Engineering Research:

Bekmuradov, V., Luk, G., and Luong, R. (2014a). Improved cellulose and organic-solvent-based 
lignocellulosic fractionation pretreatment of organic waste for bioethanol production. American Journal of Engineering Research, 3(6), 177-185. Available from http://www.ajer.org/papers/v3\%286\%29/U036177185.pdf

This study investigates the performance of the Cellulose and Organic-Solvent-based Lignocellulosic Fractionation (COSLIF) method for the pretreatment of Source-Separated Organic (SSO) waste. An improvement on the standard method of COSLIF pretreatment was developed based on lower enzyme loading and using an ethanol washing instead of acetone. It was demonstrated that a much higher glucose yield (90\% after 72 hours) was possible with this improvement, as compared to the original method, which yielded $70 \%$ in the same time frame. Evaluation of the enzymatic hydrolysate obtained from the modified COSLIF pretreatment was further examined by anaerobic fermentation with Z. mobilis $8 \mathrm{~b}$ strain. At 48 hours, ethanol concentration reached to $140 \mathrm{~g} / \mathrm{L}$, which is equivalent to $0.48 \mathrm{~g}$ of ethanol produced per gram of SSO biomass. This study demonstrated the modified COSLIF pretreatment provides a substantial improvement over the standard method in terms of enzyme savings, glucose formation, and ethanol production.

4: Comparative Ethanol Productivities of two Different Recombinant Fermenting Strains on Source-Separated Organic Waste

This chapter has been published in International Journal of Engineering Research and Applications: Bekmuradov, V., Luk, G., and Luong, R. (2014b). Comparative ethanol productivities of two different recombinant fermenting strains on source separated organic waste. International Journal of Engineering Research and Applications, 4(10), 77-82. Available from http://www.ijera.com/pages/v4no10(v5).html 
Production of biofuel such as ethanol from lignocellulosic biomass is a beneficial way to meet sustainability and energy security in the future. The main challenge in bioethanol conversion is the high cost of processing, in which enzymatic hydrolysis and fermentation are the major steps. Among the strategies to lower processing costs are utilizing both glucose and xylose sugars present in biomass for conversion. An approach featuring enzymatic hydrolysis and fermentation steps, identified as separate hydrolysis and fermentation (SHF) was used in this work. The proposed solution is to use "pre-processing" technologies, including the thermal screw press (TSP) and cellulose-organic-solvent-based lignocellulosic fractionation (COSLIF) pretreatments. Such treatments were conducted on a widely available feedstock such as source separated organic waste (SSO) to liberate all sugars to be used in the fermentation process. Enzymatic hydrolysis was featured with the addition of commercially available enzyme, Accellerase 1500, to mediate the enzymatic hydrolysis process. On average, the sugar yield from the TSP and COSLIF pretreatments followed by enzymatic hydrolysis was remarkable at $90 \%$. In this work, evaluation of the SSO hydrolysate obtained from COSLIF and enzymatic hydrolysis pretreatments on ethanol yields was compared by fermentation results with two different recombinant strains: Z. mobilis 8b and S. cerevisiae DA2416. At 48 hours of fermentation, ethanol yield was equivalent to $0.48 \mathrm{~g}$ of ethanol produced per gram of SSO biomass by Z. mobilis $8 \mathrm{~b}$ and $0.50 \mathrm{~g}$ of ethanol produced per gram of SSO biomass by $S$. cerevisiae DA2416. This study provides important insights for investigation of the sourceseparated organic (SSO) waste on ethanol production by different strains, and becomes a useful tool to facilitate future process optimization for pilot scale facilities. 
5: Kinetic Model for Ethanol Production by Recombinant S. cerevisiae Strain onSourceSeparated Organic Waste

This paper is currently under review in Computational and Structural Biotechnology Journal.

An existing kinetic model was adapted and modified to accommodate batch simultaneous saccharification and co-fermentation (SSCF) process on source-separated organic (SSO) waste by the recombinant strain, Saccharomyces cerevisiae DA2416. The model encompasses enzymatic hydrolysis and fermentation processes with competitive uptake of glucose and xylose in both stages. Enzymatic hydrolysis was featured with the addition of a commercially available enzyme, Accellerase 1500, to mediate the process. Pre-processing technologies, including the thermal screw press (TSP) and cellulose-organic-solvent-based lignocellulosic fractionation (COSLIF) pretreatments, were applied on the SSO waste to liberate fermentable sugars. On average, the sugar yields, mainly in the form of glucose and xylose, from pretreated SSO waste by enzymatic hydrolysis was $90 \%$. The kinetic model was tailored with experimentally-defined SSO parameters to evaluate the sugar and ethanol yields from SSO waste, and was found to predict ethanol production rate accurately with diminutive variance from experiments. Experimental results demonstrated that S. cerevisiae DA2416 produced more than $150 \mathrm{~g} / \mathrm{L}$ ethanol, with ethanol yield of $0.50 \mathrm{~g}$ of ethanol/g potential sugar fed, in less than 5 days with 96\% cellulose conversion. It was confirmed in this work that cellulose adsorption capacities along with hydrolysis rate constant have a high impact on sugar and ethanol formation.

This study provides important insights for investigation on the use of SSO waste for ethanol production by S. cerevisiae DA2416 and the model is proven to be a useful tool to facilitate future process optimization for pilot scale facilities. 
Chapter 6: Conclusions and Future Work Recommendations

\subsubsection{Statement of Authorship}

Chapter 1: Introduction

Valeriy Bekmuradov wrote initial draft with input from Prof. Grace Luk.

Chapter 3: Improved Cellulose and Organic-Solvent-based Lignocellulosic Fractionation

Pretreatment of Organic Waste for Bioethanol Production

Valeriy Bekmuradov, Grace Luk and Robin Luong.

Valeriy designed the experiments of this chapter with inputs from Prof. Grace Luk and Robin

Luong.

Valeriy performed all experimental work and data analysis. Writing was completed with inputs

from co-authors.

Published in 2014: American Journal of Engineering Research, 3(6), 177-185.

Chapter 4: Comparative Ethanol Productivities of two Different Recombinant Fermenting Strains on Source-Separated Organic Waste

Valeriy Bekmuradov, Grace Luk and Robin Luong.

Valeriy designed the experiments of this chapter with inputs from Prof. Grace Luk and Robin Luong. 
Valeriy performed all experimental work and data analysis. Writing was completed with inputs from co-authors.

Published in 2014: International Journal of Engineering Research and Applications, 4(10), 7782.

Chapter 5: Kinetic Model for Ethanol Production by Recombinant S. cerevisiae Strain on Source-Separated Organic waste Valeriy Bekmuradov and Grace Luk.

Valeriy designed the experiments and perform the mathematical modeling of this chapter with input from Prof. Grace Luk.

Valeriy performed all experimental work and data analysis. Writing was completed with inputs from Prof. Grace Luk.

Submitted to: Computational and Structural Biotechnology Journal 


\section{CHAPTER 3. IMPROVED CELLULOSE AND ORGANIC-SOLVENT-BASED LIGNOCELLULOSIC FRACTIONATION PRETREATMENT OF ORGANIC WASTE FOR BIOETHANOL PRODUCTION}

Abstract

This study investigates the performance of the Cellulose and Organic-Solvent-based Lignocellulosic Fractionation (COSLIF) method for the pretreatment of Source-Separated Organic (SSO) waste. An improvement on the standard method of COSLIF pretreatment was developed based on lower enzyme loading and using an ethanol washing instead of acetone. It was demonstrated that a much higher glucose yield (90\% after 72 hours) was possible with this improvement, as compared to the original method, which yielded $70 \%$ in the same time frame. Evaluation of the enzymatic hydrolysate obtained from the modified COSLIF pretreatment was further examined by anaerobic fermentation with Zymomonas mobilis 8b strain. At 48 hours, ethanol concentration reached to $140 \mathrm{~g} / \mathrm{L}$, which is equivalent to $0.48 \mathrm{~g}$ of ethanol produced per gram of SSO biomass.

This study demonstrated that the modified COSLIF pretreatment provides a substantial improvement over the standard method in terms of enzyme savings, glucose formation, and ethanol production.

\subsection{Introduction}

Pretreatment is considered one of the most expensive processing steps in the bioconversion of lignocellulosic biomass, often accounting for up to $40 \%$ of the total processing cost (Zhu et al., 2009). In addition, it greatly affects the downstream cost of operations such as 
enzymatic hydrolysis and fermentation. Additional costs resulting from inefficient pretreatment include detoxification, limited enzymatic hydrolysis rate, high enzyme loading, low product concentration, and complicated product purification. Therefore, pretreatment can be seen as a key step in limiting the feasibility of bioconversion. Pretreatment, together with enzymatic hydrolysis, is the central task of the entire bioethanol production process (Zhu et al., 2009). Evidently, all the lignocellulosic pretreatment processes experience sugar degradation and inhibitor formation. The shortfalls of the current leading lignocellulosic pretreatments can be mainly attributed to: 1) inefficiency in breaking up the orderly hydrogen bonds in crystalline cellulose, resulting in slow hydrolysis rates and low cellulose digestibility, which compromises the overall sugar yields, and 2) the presence of lignin and hemicellulose on the surface of cellulose, which is commonly thought to have the effect of restricting the accessibility of enzymes to the biomass (Zhang et al., 2007).

Cellulose and Organic-Solvent-based Lignocellulosic Fractionation (COSLIF) is a promising technology, recently developed to overcome these problems. The COSLIF pretreatment is a technology that can effectively fractionate lignocelluloses into amorphous cellulose, lignin, hemicelluloses, and acetic acid (Zhang et al., 2007; Rollin et al., 2011). This technology has been applied successfully to a broad range of substrates from agricultural to industrial waste, with inclusion of organics such as: food, paper, cardboard, plastics and yard wastes (Zhang et al., 2007; Sathitsuksanoh et al., 2009; Zhu et al., 2009; Ge et al., 2011; Sathitsuksanoh et al., 2011). The COSLIF technology has many advantages over traditional lignocellulosic pretreatments, most notably the following: modest treatment conditions at $50^{\circ} \mathrm{C}$ and atmospheric pressure; minimized degradation of sugars; no inhibitor formation; coutilization of different sugars increasing potential output; high sugar yields; fast hydrolysis rates; 
efficient solvent recycling; low usage of enzymes; and low energy consumption (Zhang et al., 2007).

The objective of this paper is to evaluate the performance of two COSLIF pretreatment methods: standard and modified, on an innovative feedstock for ethanol production, namely, source-separated organic (SSO) waste. Due to its potential for high energy content and environmental implications, SSO has been proposed as a suitable feedstock for bioethanol production (Mirzajani, 2009). It was demonstrated that the overall process of lignocellulose fractionation with the use of cellulose solvent (phosphoric acid) and organic solvent (acetone/ethanol) as pretreatment reagents is effective in hydrolyzing the sugar content of the waste (Zhang et al., 2007). In order to successfully deal with the causes of the SSO recalcitrance - breaking up orderly hydrogen bonds in the crystalline cellulose chain and removing lignin and hemicelluloses from the surface of cellulose, a standard COSLIF process was modified by using ethanol washing solvent instead of acetone and lowering enzyme loading. It allowed increase in the concentration of glucose released after enzymatic hydrolysis and to achieve the highest ethanol yield in the fermentation step. The enzymatic hydrolysis performances of the original and modified COSLIF pretreatment methods were investigated and compared in terms of their glucose yield. A scanning electron microscopy (SEM) was used to examine the supra-molecular structures of COSLIF-pretreated SSO samples for qualitative comparison.

\subsection{Materials and Methods}

The SSO waste utilized in this work was initially pre-processed mechanically, under high temperature (of approximately $120^{\circ} \mathrm{C}$ ) and pressure (over 50 bars) with a thermal screw press to 
form a semi- dry stable biomass. The thermal screw press (TSP) machine by the Aufbereitungs Technologie and System (ATS) AG, Switzerland, is a heavily built piece of processing machinery that fits into the dimensions of five meters by two meters by fifteen meters and weighs between six to seven tones, depending on the model. The machine has a twin parallel extruding screws, which run the length of the machine and pass through one to three processing chambers. These screws carry feedstock through a thermal friction processing technique created through the adjusting the friction plates that are located between the chambers. TSP is powered either by electric or diesel motors and can process biomass and other waste materials in a completely the way, such that the feedstock would grind, compress and create an effecton organic materials and carry out this function in one step. During the operation of TSP, heat is generated through friction caused by the forward pressure and turning action of the screws. Normal operating temperature run between 105 and 125 degree $C$ and pressure varies from 50 to 290 bar depending on setting needs and feedstock origin. The flexible operating principles of this machine offers great potential for processing different lignocellulosic feedstocks (Vartek Waste Management Ltd, 2005).

SSO samples were prepared as a heterogeneous substrate by blending approximately $80 \%$ organic waste with $20 \%$ woodchips from construction/demolition waste before pre-processing (Vartek Waste Management Ltd, 2005). Optimum Waste Recycling Systems, Toronto, Canada, supplied the biomass feedstock used in this work. The general flowchart of the experimental investigation is shown in Figure 3.1.

It started with the SSO waste fed to thermo-screw press and to make it homogenous. After this, the SSO samples underwent lignocellulosic fractionation with the use of a cellulose solvent (85\% phosphoric acid) and an organic solvent (either acetone or ethanol). The next step 


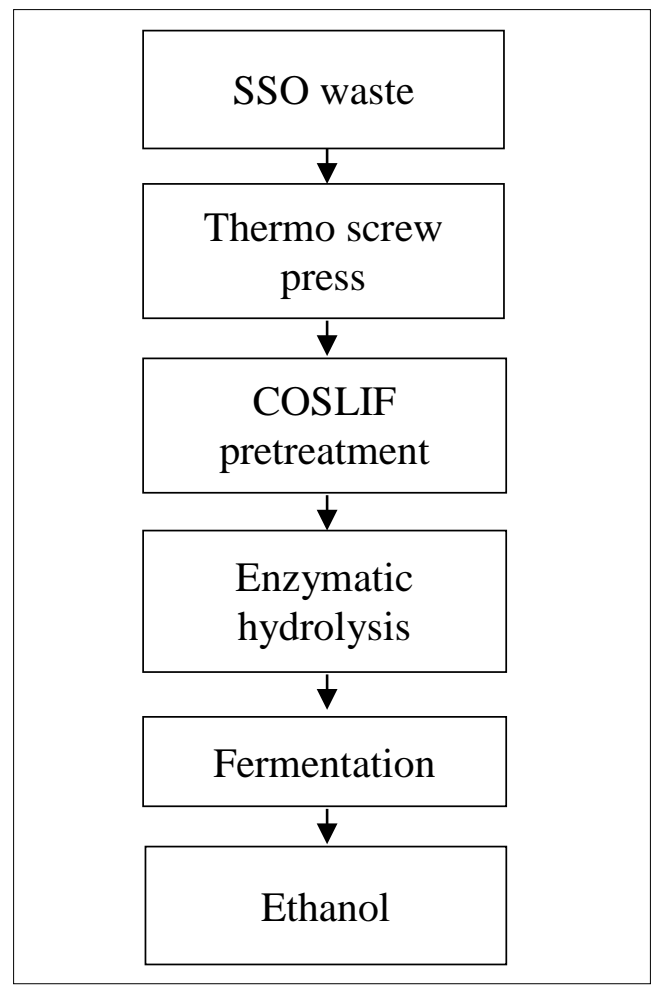

Figure 3.1: Experimental flowchart.

in the flowchart above is enzymatic hydrolysis with the addition of commercially available enzyme, Accellerase 1500, to mediate enzymatic hydrolysis process and release all fermentable sugars available for further fermentation. Accellerase 1500 is Genencor's one of the innovative enzyme products, with a significant step forward towards more cost effective, commercial scale production developed for second generation of biorefineries. It has been proven that Accellerase 1500 could successfully hydrolyze a wide range of lignocellulosic feedstocks (Retka, 2009). Accellerase 1500 enzyme used in this research was supplied by Genencore Inc., a Denisco Division, Rochester, New York, USA, as well as by the Sigma Aldrich Corp., USA.

Prior to testing, the SSO samples were oven-dried at $45^{\circ} \mathrm{C}-50^{\circ} \mathrm{C}$ for 72 hours following recommended practice (Hames et al., 2008). Five grams of dry lignocelluloses was placed in a 
$250 \mathrm{~mL}$ centrifuge bottle and then mixed with $40 \mathrm{~mL}$ of $85 \%$ concentrated phosphoric acid using a glass rod (see Appendix D for details on experimental procedures).

The solid/ liquid slurry was placed in a benchtop shaking incubator at $150 \mathrm{rpm}$ and $50^{\circ} \mathrm{C}$ $\pm 0.2^{\circ} \mathrm{C}$ for 2 hours. One hundred $\mathrm{mL}$ of ethanol was then added and mixed well. After centrifugation at $7000 \mathrm{rpm}$ at room temperature for 15 minutes, the supernatant was decanted. The solid pellet was then re-suspended by $150 \mathrm{~mL}$ of ethanol and centrifuged. The supernatant again was decanted. Next, the solid pellet was re-suspended by $150 \mathrm{~mL}$ of distilled water and centrifuge two additional times (Zhang et al., 2007; Dowe and McMillan, 2008).

Enzymatic hydrolysis experiments were conducted next in sequence in the chosen SHF approach in a benchtop shaking incubator. The separate hydrolysis and fermentation (SHF) approach was used in this study to avoid interference of samplings. The procedure for enzymatic cellulose hydrolysis was adopted from a procedure developed by the National Renewable Energy Laboratory and described in (Ehsanipour, 2010; Brown and Torget, 1996). After thawing, the treated solid pellet containing amorphous cellulose was neutralized to $\mathrm{pH} 4.8-5.0$ by $\mathrm{NH} 4 \mathrm{OH}$. Upon diluting to 20 g glucan/L based on the $27 \%$ glucose content (Ehsanipour, 2010), the sample was then brought to $50^{\circ} \mathrm{C}$ before adding $30 \mathrm{FPU} / \mathrm{g}$ glucan or $60 \mathrm{FPU} / \mathrm{g}$ glucan of Accellerase 1500. The incubator was set at $250 \mathrm{rpm}$ to keep solids in constant suspension with the temperature of $50^{\circ} \mathrm{C}$ for 72 hours. Sampling was carried out at $0,12,24,48$ and 72 hours and glucose yield was measured.

Following enzymatic hydrolysis, batch soluble sugar fermentation was carried out to determine the ethanol yields. The $Z$. mobilis $8 \mathrm{~b}$ recombinant strain was chosen for its capability to ferment glucose and to produce ethanol at high yields (Mohagheghi, 2004) and was donated by the National Renewable Energy Laboratory, Golden, Colorado, USA. Soluble sugars batch 
fermentation was performed in 250-mL serum bottles with 100-mL working volume and purged with nitrogen before being autoclaved. Temperature was maintained at $30^{\circ} \mathrm{C}-37^{\circ} \mathrm{C}$ and $\mathrm{pH}$ was controlled at $5.0-6.0$ by $1 \mathrm{M}$ potassium hydroxide $(\mathrm{KOH})$ as suggested by previous studies (Zhang et al., 2009). Each batch sugar fermentation process was carried out in triplicates on the pretreated biomass for both the standard and modified COSLIF methods.

Concentrations of glucose in hydrolysates from the COSLIF pretreated biomass and ethanol from in fermentation broths were analyzed by high performance liquid chromatography (HPLC), Bio-Rad HPX-87P column quipped with the appropriate guard column. All concentrations were reported as per liter volume basis. Percent theoretical ethanol yield was calculated (Dowe and McMillan, 2008):

$$
\% \text { Theoretical ethanol yield }=\frac{[\text { EtOH }] f-[\text { EtOH }] i}{0.51 \cdot(f \cdot[\text { Biomass }] \cdot 1.111) \cdot 100}
$$

where $[\mathrm{EtOH}]_{\mathrm{f}}$ - ethanol concentration at the end of fermentation, $(\mathrm{g} / \mathrm{L})$; $[\mathrm{EtOH}] \mathrm{i}$ - ethanol concentration at the beginning of fermentation, (g/L); [Biomass] - the dry biomass concentration at the beginning of fermentation, $(\mathrm{g} / \mathrm{L}) ; \mathrm{f}$ - the cellulose fraction of dry biomass $(\mathrm{g} / \mathrm{g}) ; 0.51$ - the conversion factor for glucose to ethanol; 1.111 - conversion factor for cellulose to equivalent glucose.

Supra-molecular structures of the intact and pretreated SSO samples were examined by scanning electron microscope, as described elsewhere (Zhang et al., 2006; Selig et al., 2007). A scanning electron microscope (SEM) is a type of electron microscope that produces images of a sample by scanning it with a focused beam of electrons. The electrons interact with atoms in the sample, producing various signals that can be detected and that contain information about the sample's surface topography and composition. The electron beam is generally scanned in a 
rectangular pattern of image, and the beam's position is combined with the detected signal to produce an image. SEM can achieve resolution better than 1 nanometer. Samples can be observed in high and low vacuum, and in wet conditions. A SEM was kindly provided by the Ryerson University Analytical Center, Toronto, Canada.

\subsection{Results and Discussion}

A detailed quantitative assessment on the composition of SSO waste was carried out (Ehsanipour, 2010) and adopted for further investigation in this study. The SSO samples, contained $20 \%$ woodchips, were already pretreated by the thermal screw machine. The woodchips were typically Douglas fir wood waste originated from home construction furniture, flooring, cabinet, and doors. All sharp foreign matter such as metal needles, plastic and rubber wastes, and broken glasses were collected and removed, as much as it was possible. The dried SSO biomass was sent to MBI International, the Michigan State University Foundation, for grinding and determination of polymeric sugars content. The results are summarized in Table 3.1. It turned out that those essential polymeric sugars made up $41.3 \%$ in oven dried SSO samples, including: $27 \%$ glucan, $5.4 \%$ xylan, $5.7 \%$ mannan, $1.2 \%$ arabinan, and $1.2 \%$ of galactan, which were a good starting point for enzymatic hydrolysis followed by fermentation. It was found that the SSO samples were acidic (pH of 5.0-5.5) and had the highest content of the food waste, just about $80 \%$ of total waste of samples. Comparison between pretreated and nontreated SSO validated the high recalcitrant nature of lignocellulosic fraction of biomass as suggested by Zhang et al. (2007), and which was in agreement with other works (Zhang et al., 2009; Zhu et al.,2009; Rollin et al., 2011). 
Table 3.1: Compositional analysis of source-separated organic samples

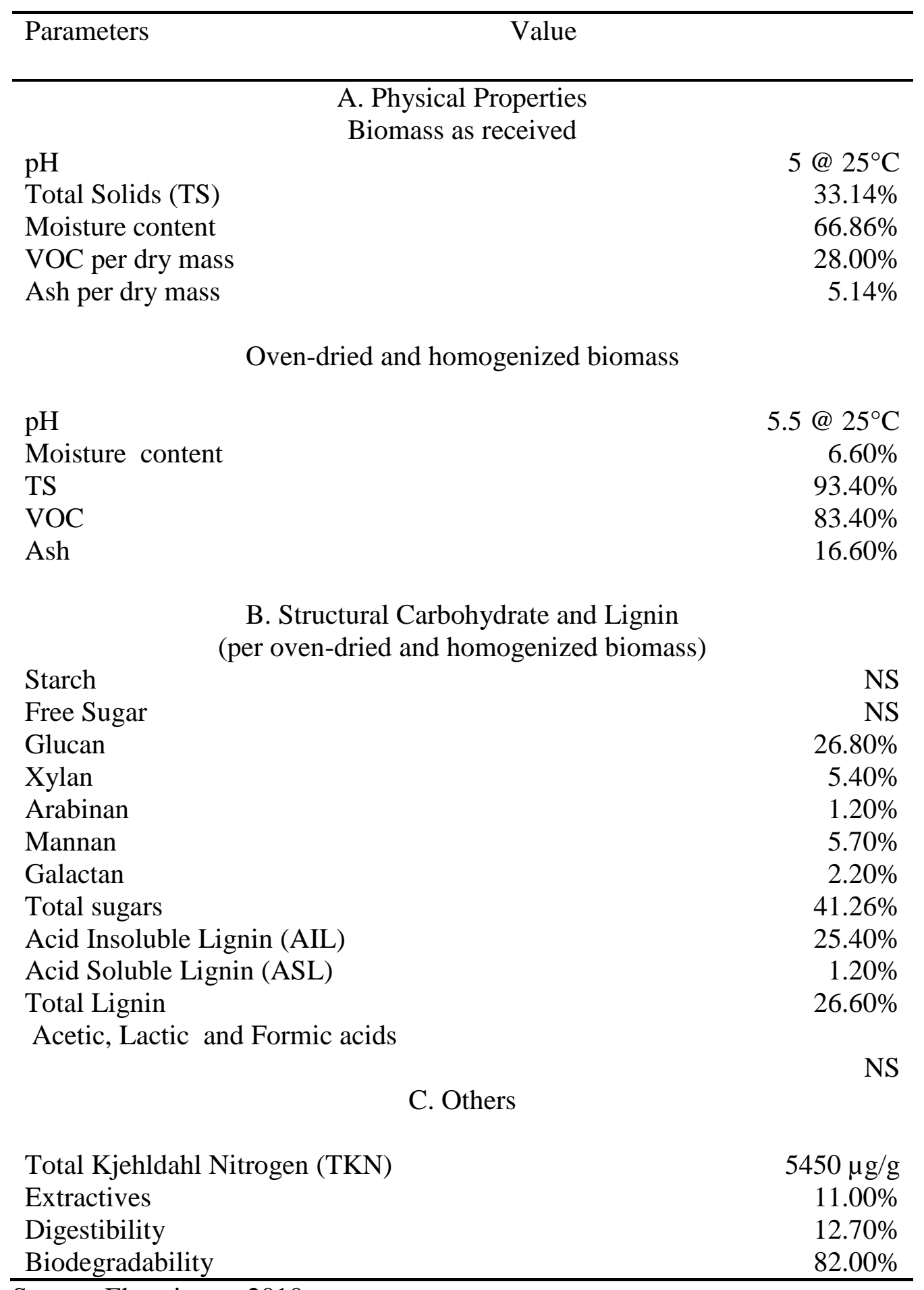

Source: Ehsanipour, 2010

NS - not significant 


\subsubsection{Glucose Yield}

Results obtained from COSLIF washing (shown in Figure 3.2) with concentrated phosphoric acid and acetone reagent generated a significant glucose yield of about $70 \%$, in the first few trials.

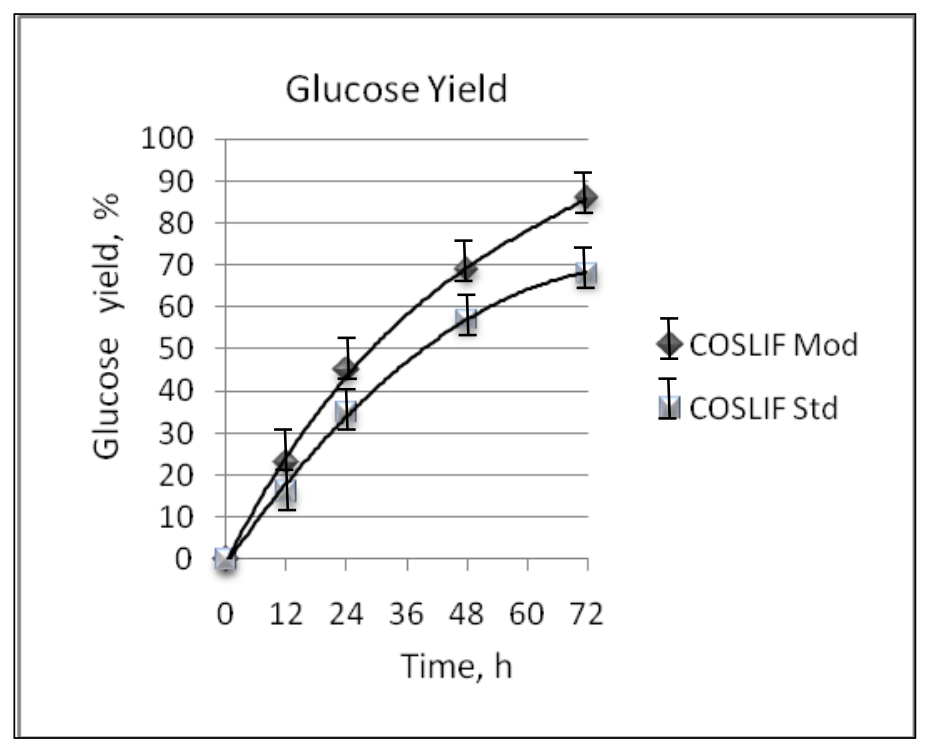

Figure 3.2: Glucose yields of standard and modified COSLIF pretreatment performed at $50^{\circ} \mathrm{C}$ for 72 hours

However, acetone is a more toxic reagent and it is less safe to use than ethanol. The cost of using acetone is higher than that of ethanol and during the recovery of the reaction's byproducts more energy is consumed when acetone is used as the reagent. In addition, pretreatment with acetone must be performed under extremely stringent and efficient conditions due to the volatility of acetone. Ethanol, on the other hand, is less corrosive and can be easily recovered by distillation under milder conditions. Therefore, after extensive trials and investigations, some changes were made to further improve the efficiency of COSLIF pretreatment to obtain a higher 
glucose yield. The major change made to the original standard method of COSLIF pretreatment was to omit acetone altogether and use $95 \%$ (v/v) ethanol as the organic solvent instead. Another was changing enzyme loading from 60FPU to 30FPU. As a result of these changes, the glucose yield increased to approximately $90 \%$ (Figure 3.2). Additionally, only 50\% of the original volume of ethanol was needed to replace the acetone.

\subsubsection{Enzymatic Hydrolysis}

Figure 3.3 shows the glucose digestibility profiles over a course of 72 hours for the SSO samples treated by the standard and modified COSLIF methods as well as non-treated samples.

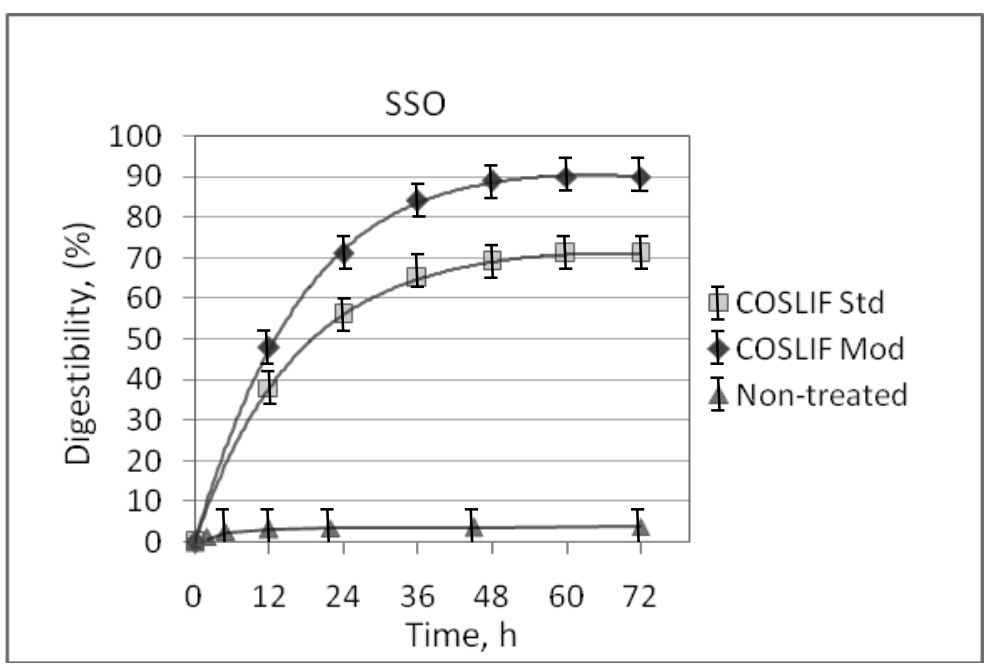

Figure 3.3: Time trend of glucose digestibility from the non-treated to standard and modified COSLIF pretreated samples

High glucan digestibility of the pretreated SSO was accredited to drastic changes in the supramolecular structure of the biomass before and after the COSLIF pretreatment, observed by 
the SEM in this study. Typical COSLIF pretreatment conditions were used, namely $50^{\circ} \mathrm{C}$ and atmospheric pressure with a pretreatment time from 30 to 60 minutes, depending on the type of feedstock. Although diverse feedstocks showed great variations in enzymatic digestibility, suggesting that their different recalcitrant structures confer variable resistance to enzymes, the use of concentrated phosphoric acid at $50^{\circ} \mathrm{C}$ can efficiently dissolve them so to erase their inherent structure difference and result in an amorphous biomass with similar high-accessibility (Rollin et al., 2011; Sathitsuksanoh et al., 2011). As a result, COSLIF-pretreated biomass feedstock exhibited similar enzymatic glucan digestibility regardless of their sources (Sathitsuksanoh et al., 2011). When concentrated phosphoric acid was used as the cellulose solvent, it should be used at $50^{\circ} \mathrm{C}$ or lower to avoid extensive hydrolysis of polymeric carbohydrates and sugar degradation.

The enzymatic glucose digestibility for pretreated COSLIF samples was calculated as described by Zhang et al. (2007). With high enzyme loading, 60FPU and acetone washing, the glucose digestibility of the pretreated standard COSLIF sample was approximately $70 \%$ as presented in Figure 3.3 above. With a lower enzyme loading, 30FPU and ethanol washing, it reached 90\% digestibility after 36 hours. This suggests that by removing hemicelluloses and lignin barriers, there was an increase in accessibility to the cellulose change by the cellulobiose, while also reducing the competitive inhibition of xylan to endo-glucanase. Data from this study on the hydrolysis rates and digestibility were comparable to the range (90\%-95\%) cited in other scientific papers (Mosier et al., 2005; Wyman et al., 2005). 


\subsubsection{Fermentation}

Fermentation is the final step in evaluating the overall process of cellulosic ethanol production. The effectiveness of the enzymatic hydrolysis was gauged by assessing the potential inhibitory factors and effects of fermentation. These results can be found in the following section. A genomic DNA-integrated glucose and xylose co-fermenting strain, Z. mobilis 8b recombinant strain was used due to its ability to ferment glucose and xylose to produce ethanol at high yields (Mohagheghi et al., 2004). The microbe was developed and evaluated by the NREL on a broad range of agricultural biomass and can convert sugars to ethanol more rapidly as compared to other species.

Besides the major changes during the COSLIF pretreatment process, some minor improvements in the fermentation procedure were also made and they undoubtedly affected overall efficiency of the final ethanol output. These improvements were as follows: a serum bottle with a crimp top was used instead of an Erlenmeyer flask with stopper for better air-tight seal; a flushing serum bottle with nitrogen was used to maintain anaerobic conditions prior to fermentation; a direct transfer technique was exploited to move concentrated Z. mobilis 8b cells from an inoculums tube to a serum bottle; and a growth curve was developed for the Z. mobilis 8b strain prior to fermentation tests which was important in order to identify the OD (optical density) range in the exponential phase of a curve. The OD values in the exponential phase were vital in determining the time to harvest the cells to start the fermentation process. There were two protocols that could be employed for harvesting the cells to start the fermentation process: 1) use of a direct transfer (10\%) to the main fermentation bottle or 2 ) use of concentrated cells by centrifuging in a centrifuge tube and then re-suspending the cells in a hydrolysate before transferring it back into the fermentation bottle. The second protocol was chosen because the 
inoculated seed media contained not only cells but also a large amount of glucose sugar which would be transferred into the fermentation bottle. Unless distilled deionized water (DDW) blank was created, this would result in false and inaccurate HPLC readings of glucose and ethanol concentrations.

The high ethanol yield shown in Figure 3.4 indicated that very little inhibitors were present in the hydrolysates that were pretreated by the modified COSLIF method. Depending on feedstock and process, the actual yield could be anywhere from $60 \%$ to $100 \%$ of the theoretical yield. Achieving a high yield may be costly compared to lower yield processes that are often more cost effective.

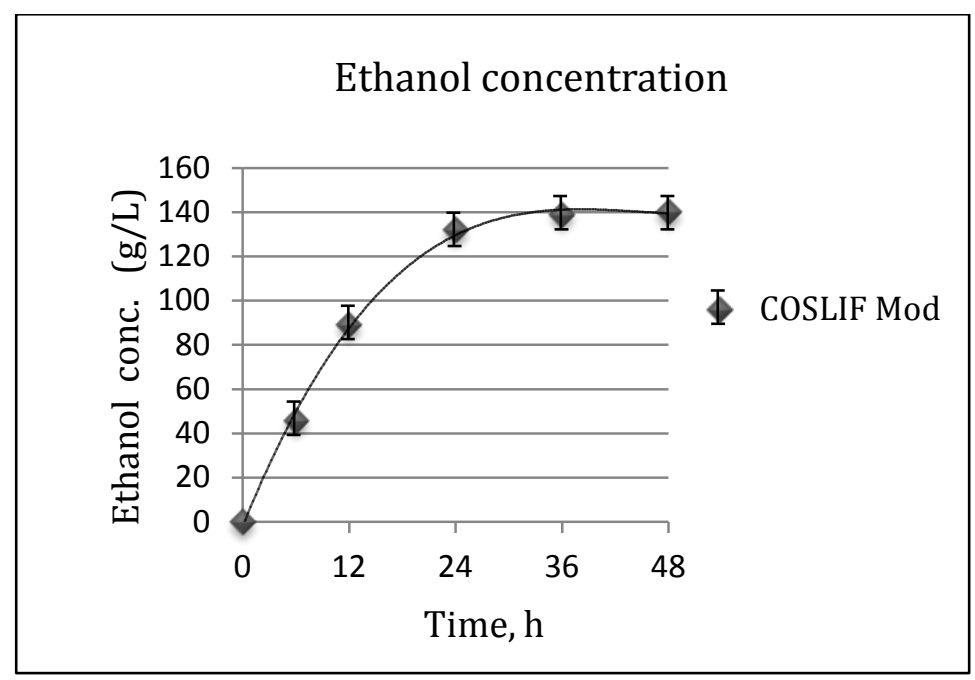

Figure 3.4: Ethanol concentration from modified COSLIF pretreated samples

The ethanol concentration rate was calculated on the basis of sugars consumed as described by South et al. (1995), and it yielded in $132.1 \mathrm{~g} / \mathrm{L}$ for the pretreated samples by the modified COSLIF method after 24 hours. At 48 hours, the ethanol concentration reached 140 $\mathrm{g} / \mathrm{L}$, which is equivalent to $0.48 \mathrm{~g}$ ethanol/g biomass or $94 \%$ of the theoretical ethanol yield. As 
per this work, percent theoretical ethanol yield was calculated accordingly (Dowe and McMillan, 2008). Although the ethanol concentration for some samples seemed to be fluctuating from time to time, over $90 \%$ ethanol yield can be attributed to the high accessibility of the pretreated cellulosic materials and low presence of lignin.

\subsubsection{Comparison with Constructed Sugar Model}

In a further series of experimental evaluations, enzymatic hydrolysate obtained from both COSLIF pretreatments by batch culture fermentation with Z. mobilis 8b strain were compared with constructed sugar model (glucose/xylose ratio as 5:1) in SSO substrate. In a constructed model, after 24 hours, $100 \%$ of glucose and $40 \%$ of xylose were consumed. While in the enzymatic hydrolysate, pretreated by COSLIF with ethanol washing reagent, the fermentation also advanced rapidly and $90 \%$ glucose and $40 \%$ xylose were also consumed, in the enzymatic hydrolysate, pretreated by COSLIF with acetone washing reagent, the fermentation advanced slowly and $45 \%$ of glucose remained unused in the same period of time. Low bacterial activity in the fermentation process of SSO hydrolysates may be attributed to many factors including: longer lag phase for $Z$. mobilis $8 \mathrm{~b}$ strain as the adaptation time to growth condition, low growth rate on SSO hydrolysates, unavoidable contamination during sample preparations, lack of nutrients, and presence of inhibitors.

\subsubsection{Qualitative Analysis}

As per qualitative comparison, SEM images of oven-dried SSO substrate before and after pretreatment were conducted in collaboration (Ehsanipour, 2010) and provided in Figure 
3.5.These images show the appearance of SSO before grinding $-1-1$, after grinding $-2-1$, and after COSLIF pretreatment - 3-1. Each pretreatment (physical and chemical) process changed the structure of the SSO biomass. Before the pretreatment, the plant cell wall structures of the SSO and cellulose fibers were clearly identified. The SEM images from 1-1 and 2-1present changes in particle size.

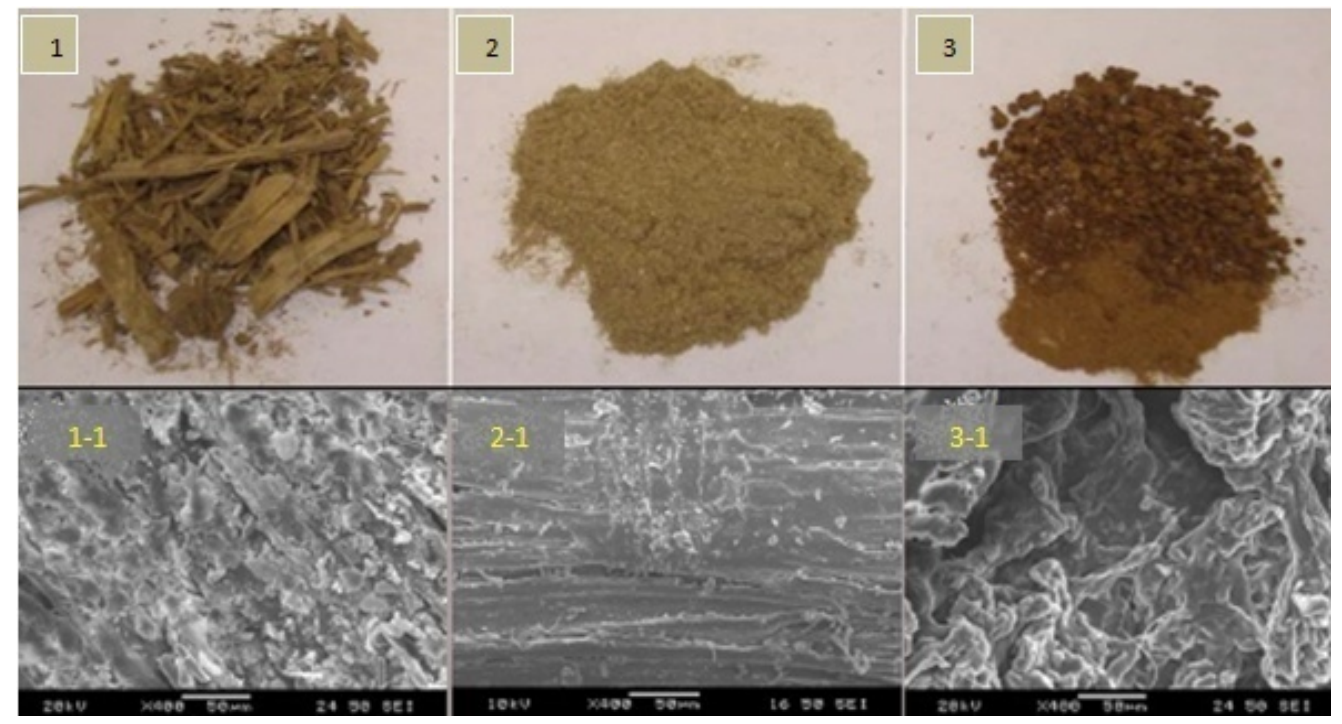

Figure 3.5: Scanning electron microscopy images of source-separated organic waste Source: Ehsanipour (2010)

The image from 3-1 shows all fibrous structures completely disrupted after pretreatment, indicating phosphoric acid and ethanol washing not only disrupted all linkages among cellulose, hemicelluloses and lignin, but also disrupted the orderly hydrogen bonds among glucose chains. These qualitative images are consistent with the images from similar studies (Zhang et al., 2006; Zhu et al., 2009). 


\subsection{Conclusions}

The SSO waste samples utilized in this research were pre-processed by the thermal screw press (TSP) and further used as a substrate for all enzymatic hydrolysis and fermentation processes.SSO has an excellent potential to be utilized as a feedstock for ethanol production due to its high fermentable sugar content, $40 \%$ - 42\%. On the other hand, SSO has high lignin content, which is slightly above $26 \%$ per kg of dried feedstock emphasizes need for pretreatment.

COSLIF pretreatments were applied for cellulose extraction. Results indicate the percent glucose conversion was considerable for the modified COSLIF method with significant glucose yield, just above 90\%. This study also demonstrated and confirmed that the COSLIF pretreatment can be carried out on this innovative type of biomass with a relatively high percentage of glucose and ethanol yields, when certain modifications are made to the process: a) the efficiency of using modified COSLIF pretreatment was improved by $20 \%$ using ethanol instead of acetone; b) using serum bottle with crimp top in fermentation process instead Erlenmeyer flask with stopper for air tight seal; c) using concentrated cells during fermentation phase instead of direct transfer mode to avoid false HPLC reading; and d) decrease enzyme loading from 60FPU to 30FPU for cost reduction. However, there are still some aspects of the process that need further investigation. For example, biomass size reduction by milling or grinding is energy intensive and costly which will affect the total cost of ethanol production. The extrusion process alone could disrupt the lignocellulosic structure, which would enable enzymes to gain access and attack the carbohydrates (Alvira et al., 2010). Detailed investigation on ethanol concentration and yield is still required. It was hypothesized that the large variations of ethanol concentration in this study were caused by interference of samplings. However, this has yet to be proven. 
For supporting materials on this chapter please refer to Appendix A "Glucose and ethanol yields calculations”. 


\title{
CHAPTER 4. COMPARATIVE ETHANOL PRODUCTIVITIES OF TWO DIFFERENT RECOMBINANT FERMENTING STRAINS ON SOURCE- SEPARATED ORGANIC WASTE
}

\begin{abstract}
Production of biofuel such as ethanol from lignocellulosic biomass is a beneficial way to meet sustainability and energy security in the future. The main challenge in bioethanol conversion is the high cost of processing, in which enzymatic hydrolysis and fermentation are the major steps. An approach featuring enzymatic hydrolysis and fermentation steps, identified as separate hydrolysis and fermentation (SHF) is proposed to eliminate this problem. The solution is to use "pre-processing” technologies, including the thermal screw press (TSP) and cellulose-organic-solvent-based lignocellulose fractionation (COSLIF) pretreatments. Such treatments are conducted on ground-breaking feedstock such as source separated organic waste (SSO) to liberate all sugars to be used in the fermentation process. TSP and COSLIF pretreatments followed by enzymatic hydrolysis were applied on the SSO to unlock fermentable sugars (glucose and xylose) for ethanol production. Enzymatic hydrolysis was featured with addition of commercial available enzyme, Accellerase 1500, to mediate enzymatic hydrolysis process. On average, the sugar yield from the TSP and COSLIF pretreatments followed by enzymatic hydrolysis was remarkable at $90 \%$. In this work, evaluation of the SSO hydrolysate obtained from COSLIF and enzymatic hydrolysis pretreaments on ethanol yields was compared by fermentation results with two different recombinant strains: Zymomonas mobilis 8b and Saccharomyces cerevisiae DA2416. At 48 hours of fermentation, ethanol yield was equivalent to $0.48 \mathrm{~g}$ of ethanol produced per gram of SSO biomass by Z. mobilis $8 \mathrm{~b}$ and $0.50 \mathrm{~g}$ of ethanol produced per gram of SSO biomass by S. cerevisiae DA2416.
\end{abstract}


This study provides important insights for investigation of the source-separated organic (SSO) waste on ethanol production by different strains and becomes a useful tool to facilitate future process optimization for pilot scale facilities.

\subsection{Introduction}

Ethanol production from lignocellulosic biomass has a potential to be a viable replacement or supplement for fossil fuel, but the current cost of conversion is a major bottleneck for commercial application (U.S. Department of Energy, 2006). The price for ethanol remains as high as $\$ 2.75$ per gallon motivating further research (Collins, 2007). By contrast the average price for regular, unleaded gasoline in the USA is currently hovering around \$3.9 per gallon with expectation for it to rise even more (U.S. Department of Energy, 2014). It became apparent that in efforts to reduce the production costs of ethanol, improvements in several areas of biofuel production including feedstock, price design, and enzymes are required. At the present time, there are at least two methods of ethanol production from lignocellulose that are in advanced phases of development: enzymatic hydrolysis and biomass fermentation. Neither process generates toxic emissions while producing the end product, ethanol. The technology is relatively new and exists in pilot configurations where testing is ongoing. While today ethanol is mostly produced from starch contained in grains such as corn, sugarcane and grain sorghum, it can also be produced from cellulose which is mainly present in non-food products. Currently, lignocellulosic feedstock is the most abundant biomass, which has attracted considerable attention and is often a major or the sole component of different waste streams from various 
industries including agriculture, forestry, and municipalities’ wastes (Taherzadeh and Karimi, 2008).

Today’s bioethanol technology has offered sustainable approaches to the problem with municipal solid waste (MSW) by focusing on utilization of organic fraction of solid waste and agriculture residue in order to reduce wastes and avoid conflicts between human food and industrial use of crops. Organic fraction of solid waste has given a new perspective to the industry by defining an innovative system for converting trash into bioethanol reducing the amount of waste piling up in landfills, while displacing a large fraction of the fossil fuels to power vehicles. Biomass such as processed source separated organic (SSO) waste is particularly attractive in one context since it is widely available at a negative cost and has many other environmental benefits. It provides a good alternative fuel in terms of green-house gas emissions, reduction of farmland's depletion, and diminutive of generated waste.

Ethanol yield and productivity are the key parameters in the production of biofuel from biomass and wastes. The fermentation of xylose-to-ethanol is important in biomass-to-ethanol process since it can increase ethanol yield up to 50\% (Hinman et al., 1989). Several strains have been engineered to ferment xylose to ethanol (Hahn-Hagerdal et al., 1993; McMillan, 1994; Mohagheghi et al., 2004). Among them are Zymomonas mobilis, Saccharamyces cerevisiae, and Pitchia stipulus. The first two abovementioned strains met the selection criteria which were based on several fermentation characteristics considered to be essential for biomass-to-ethanol conversion (Picataggio et al., 1994; Zhang et al., 1995).

The purpose of this study was a comparison of the growth and fermentation performances of pretreated source-separated organic (SSO) waste on ethanol productivities of two glucose/xylose utilizing recombinant strains: Z. mobilis 8b and S. cerevisiae DA2416. The 
feasibility of the SSO as a potential feedstock for ethanol production has been widely demonstrated (Mirzajani, 2009; Percy, 2009; Ehsanipour, 2010; Faye, 2010; Luong, 2012;

Bekmuradov et al., 2014a). Before pretreatment, a compositional characterization of preprocessed SSO samples collected at the City of Toronto, Ontario, Canada for a ten-month period was carried out (Mirzajani, 2009).

\subsection{Materials and Methods}

The SSO waste samples intended in this research were pre-processed mechanically under high temperature and pressure by the thermal screw press (TSP) and then used as a substrate for all enzymatic hydrolysis and fermentation processes. Moreover, the SSO waste samples were made as a heterogeneous substrate of demolished construction waste blended with approximately $20 \%$ of woodchips and $80 \%$ organic "green bin" waste and pre-processed accordingly (Vartek Waste Management Ltd, 2005). Prior to testing the SSO waste was oven dried at $45^{\circ} \mathrm{C}-50^{\circ} \mathrm{C}$ for 48 hours.

The next step encompassed lignocellulosic fractionation by cellulose-solvent (phosphoric acid) and organic-solvent (ethanol). Five grams of dry lignocelluloses was placed in a $250 \mathrm{~mL}$ centrifuge bottle and then mixed with $40 \mathrm{~mL}$ of $85 \%$ concentrated phosphoric acid using a glass rod. The solid/ liquid slurry was placed in a benchtop shaking incubator at $150 \mathrm{rpm}$ and $50^{\circ} \mathrm{C} \pm$ $0.2^{\circ} \mathrm{C}$ for 2 hours. One hundred $\mathrm{mL}$ of ethanol was then added and mixed well. After centrifugation at $7000 \mathrm{rpm}$ at room temperature for 15 minutes, the supernatant was decanted. The solid pellet was then re-suspended by $150 \mathrm{~mL}$ of ethanol and centrifuged. The supernatant 
again was decanted. Next, the solid pellet was re-suspended by $200 \mathrm{~mL}$ of distilled water and centrifuge two times and stored in a freezer for a short period of time (Rollin et al., 2011).

Enzymatic hydrolysis experiments were carried out with addition of commercially available enzyme, Accellerase 1500. After thawing, the treated solid pellet containing amorphous cellulose was neutralized to $\mathrm{pH} 4.8-5.0$ by $\mathrm{NH}_{4} \mathrm{OH}$. The SSO samples were then brought to $50^{\circ} \mathrm{C}$ before adding $30 \mathrm{FPU} / \mathrm{g}$ glucan of Accelerase 1500 . Both the $\mathrm{pH}$ value and temperature described were the optimum conditions for the Accelerase 1500 enzyme to mediate hydrolysis and release fermentable sugars as much as possible. The hydrolysis experiment was conducted in the benchtop shaking incubator. The incubator was set at 250 rpm to keep solids in constant suspension with the temperature of $50^{\circ} \mathrm{C}$ for 72 hours. Samples were taken for sugar content at specified times: $0,12,24,48$ and 72 hours to measure sugar content. The relevant composition of the SSO was 33\% (w/v) glucose, 19\% (w/v) xylose and 3\% (w/v) acetic acid.

Following enzymatic hydrolysis, batch soluble sugar fermentation was carried out to evaluate ethanol yields by performance of two different recombinant strains: Z. mobilis $8 \mathrm{~b}$ and $S$. cerevisiae DA2416. Soluble sugars batch fermentation was performed in $250 \mathrm{~mL}$ serum bottles with $100 \mathrm{~mL}$ working volume and purged before being autoclaved. Temperature was maintained at $30^{\circ} \mathrm{C}$ and $\mathrm{pH}$ was controlled at 6.0 by $1 \mathrm{M}$ potassium hydroxide $(\mathrm{KOH})$ as suggested by previous study (Zhang et al., 2009).

Compositional analysis of the samples in duplicates for ethanol concentrations was carried out at 0, 12, 24 and 48 hours by HPLC. The metabolic ethanol yield, Ym was calculated as a mass of ethanol produced per mass of sugar consumed. The process ethanol yield, Yp was obtained by dividing the ethanol concentration by total sugar concentration in the feed medium. 
The volumetric ethanol productivity was derived by ratio of ethanol concentration and time taken to complete fermentation (48 hours).

\subsection{Results and Discussion}

Due to its potential for industrial application, the SSO waste was chosen as the substrate to evaluate the values on sugar and ethanol yields by fermentation using Z. mobilis $8 \mathrm{~b}$ and $S$. cerevisiae DA2416 strains. Detailed quantitative assessment on the composition of SSO waste was completed prior to this study (Mirzajani, 2009), and the results are presented in Table 4.1.

As seen in Table 4.1, approximately, more than half of the original sample is composed of moisture. Essential polymeric sugars in an oven dried SSO samples included: 33\% glucose, $19 \%$ xylose, and about $9 \%$ of other sugars and $23 \%$ of lignin. These homogeneous samples with $\mathrm{pH}$ at 5.2-5.5 had approximately $80 \%$ of the food waste and a $20 \%$ of wood chips.

Enzymatic hydrolysis and fermentation experiments were next in the line to be conducted in sequence in the chosen SHF approach. The whole process usually takes five days to complete. The SSO samples pretreated by concentrated phosphoric acid (85\% w/w) and ethanol (95\% v/v) were hydrolyzed fast and glucan digestibility was found to be $72 \%$ after 24 hours and $90 \%$ after 72 hours. The high glucan digestibility seen in Figure 4.1 was achieved for the COSLIFpretreated SSO with addition of 30FPU/ g glucan of Accelerase 1500.

This result was mainly attributed to drastic changes in surface morphology of intact and COSLIF-pretreated SSO samples. The intact SSO has obviously maintained its tight micro-fibril structure, while a COSLIF-pretreated sample evidenced homogeneous biomass as seen in our previous work (Bekmuradov et al., 2014a). The enzymatic glucose digestibility for pretreated 
Table 4.1: Compositional analysis of SSO sample

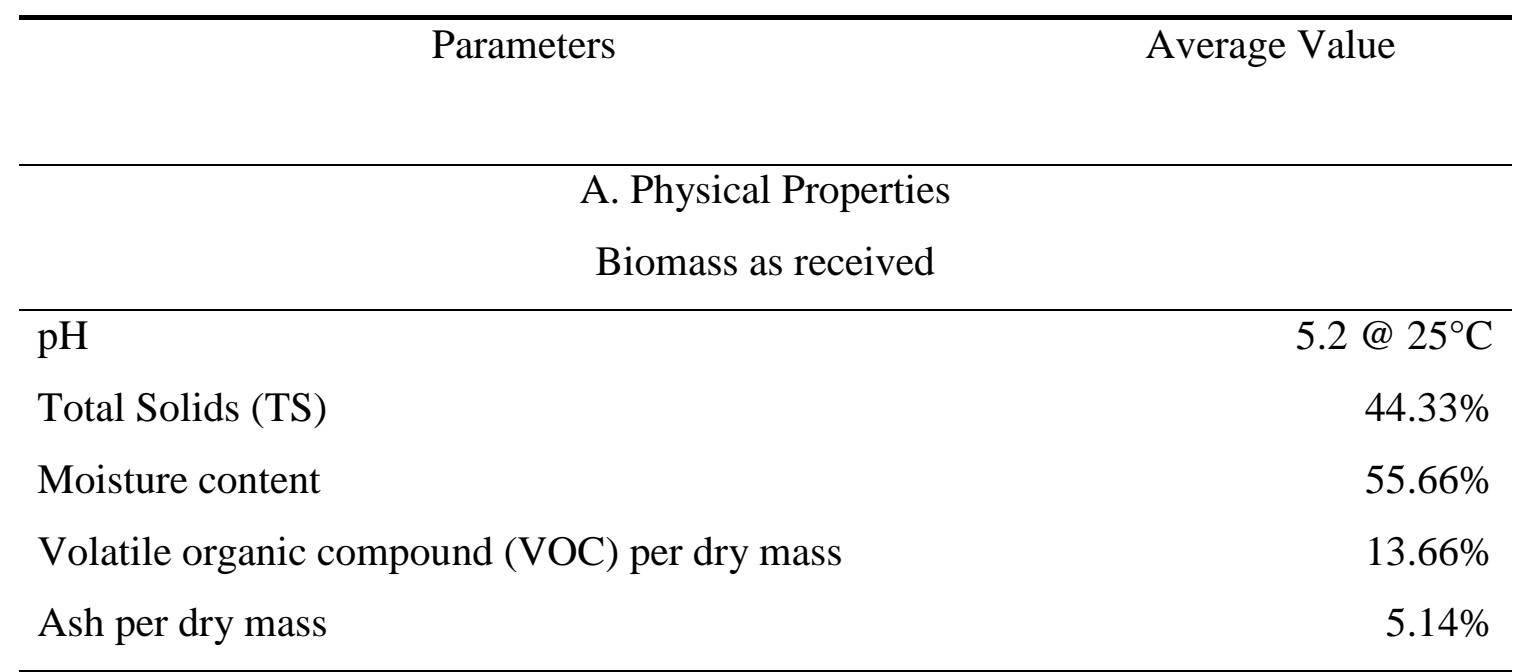

Oven-dried and homogenized biomass

\begin{tabular}{lr}
\hline $\mathrm{pH}$ & $5.5 @ 25^{\circ} \mathrm{C}$ \\
Moisture content & $6.60 \%$ \\
TS & $93.40 \%$ \\
VOC & $86.33 \%(\mathrm{TS})$ \\
Ash & $13.60 \%$ (TS) \\
\hline
\end{tabular}

B. Sugars and Lignin

(per oven-dried and homogenized biomass)

\begin{tabular}{lr}
\hline Glucose & $31 \%$ \\
Xylose & $19 \%$ \\
Other sugars & $9 \%$ \\
Total sugars & $59 \%$ \\
Total Lignin & $23 \%$ \\
\hline
\end{tabular}

C. Others

\begin{tabular}{lr}
\hline Total Kjehldahl Nitrogen (TKN) & $9198 \mu \mathrm{g} / \mathrm{g}$ \\
Extractives & $7 \%$ \\
Calorific value & $16961.6 \mathrm{kj} / \mathrm{kg}$ \\
\hline
\end{tabular}




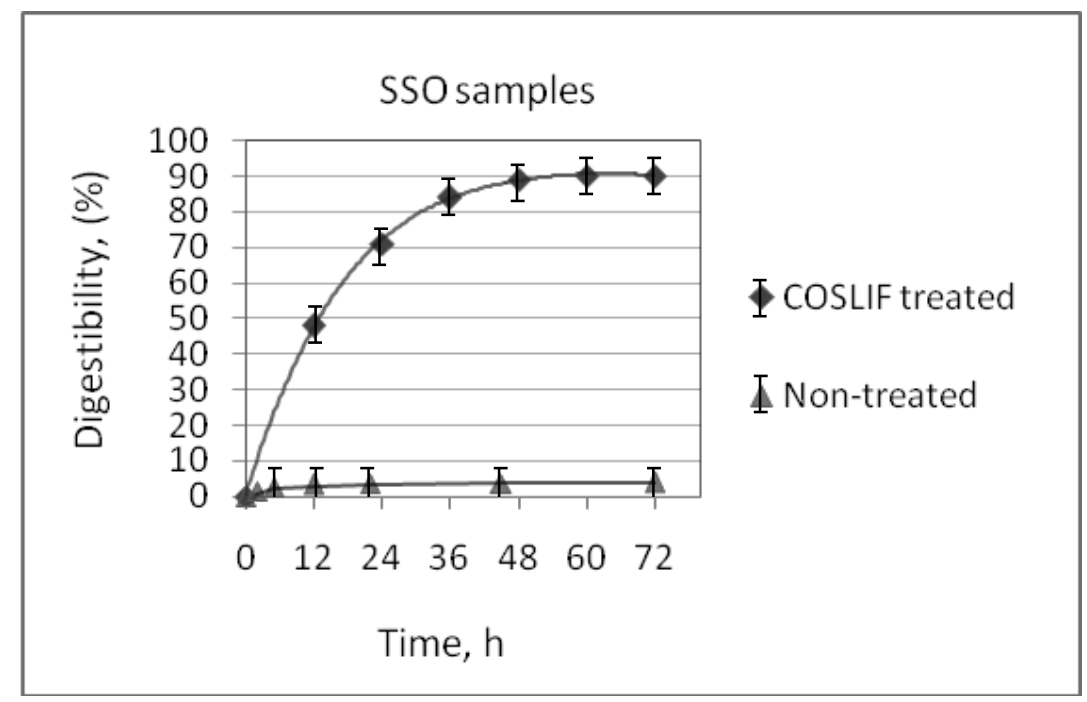

Figure 4.1: Glucan digestibility profiles for COSLIF treated and untreated SSO samples

COSLIF samples was calculated as described by Zhang et al. (2007). We hypothesized that almost all lignin have been removed from SSO waste sample during COSLIF and enzymatic hydrolysis phases. But it would be impractical to completely wash cellulose solvents out, as it requires a large amount of water. Negative effects of residual lignin on enzymatic hydrolysis may contribute to 1) enzyme adsorption by lignin, 2) obstruction of lignin on the surface of cellulose to that point when enzyme are not able to access cellulose (Collins, 2007; Zhu et al., 2009).

In a separate series of experimental evaluation, enzymatic hydrolysate obtained from COSLIF pretreament by batch culture fermentation with Z. mobilis 8b strain, was compared with S. cerevisiae DA2416. Figure 4.2 shows the glucose and xylose consumption trajectories for fermentation of the SSO pretreated samples. 
As seen from Figure 4.3 both strains exhibited almost the same value of ethanol yields based on sugar consumed $(0.48 \mathrm{~g} / \mathrm{g}$ and $0.50 \mathrm{~g} / \mathrm{g})$ and the process yield on the total initial sugar concentration was $0.48 \mathrm{~g} / \mathrm{g}$ for $Z$. mobilis $8 \mathrm{~b}$ and $0.49 \mathrm{~g} / \mathrm{g}$ for S. cerevisiae DA2416 (Table 4.2). After 72 hours, glucose is completely decomposed, while a small amount of xylose remains. Results show the main substrate for Z. mobilis 8b is glucose, while S. cerevisiae DA2416 decompose both glucose and xylose. Therefore the production of ethanol is higher for the

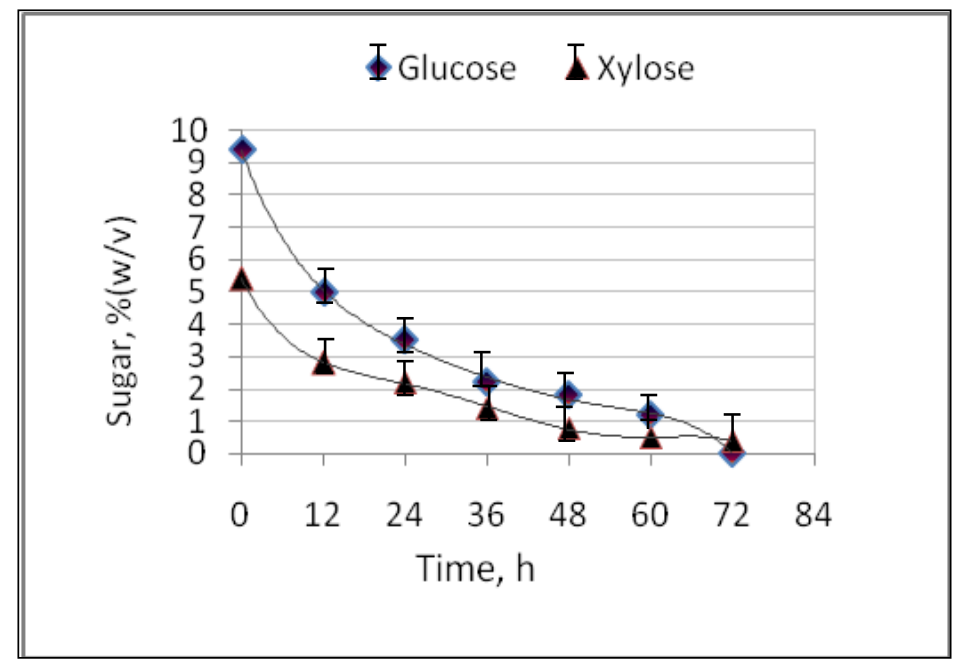

Figure 4.2: Sugar consumption profiles of the SSO pretreated hydrolysates during fermentation phase

S. cerevisiae DA2416 strain after glucose is used up. The significantly better performance of $S$. cerevisiae DA2416 compared to Z. mobilis 8b suggests a possible role of inhibitors other than acetic acid on bacterial growth in fermentation phase, for example phenolic compounds from lignin and etc. It is both well-known and documented (South et al., 1995; Rogers et al., 1997; Lawford and Rousseau, 2000) that ethanol is an inhibitor to xylose utilization by Z. mobilis 8b with ethanol concentration of 5.5\%-6\% (w/v) causing complete deceleration of the process. 
In further fermentation assays with the Z. mobilis 8b strain, after 48 hours, $100 \%$ of glucose and $40 \%$ of xylose were consumed. On the other hand, in the enzymatic hydrolysate with S. cerevisiae DA2416, fermentation advanced more rapidly, with $100 \%$ glucose and $60 \%$ xylose consumed after the same period of time. The growth and fermentation parameters of this work are summarized in Table 4.2.

Table 4.2: Growth and fermentation parameters

\begin{tabular}{lcc}
\hline \multicolumn{1}{c}{ Strains } & Z. mobilis & S. cerevisiae \\
& $8 \mathrm{~b}$ & DA2416 \\
\hline Total amount of sugar, \% (w/v) & 14.8 & 14.8 \\
Glucose, \% (w/v) & 9.5 & 9.5 \\
Xylose, \% (w/v) & 5.3 & 5.3 \\
Acetic acid, \% (w/v) & 1.0 & 1.0 \\
Process yield, g/g & 0.48 & 0.49 \\
Metabolic yield, g/g & 0.48 & 0.50 \\
${ }^{1}$ Productivity, g/L·h & 0.88 & 0.92 \\
Ethanol yield, g/L & 140 & 152 \\
\hline $\begin{array}{l}{ }^{1} \text { Productivity data was based on fermentation time of } 48 \text { hours } \\
\text { Process yield was based on available sugars } \\
\text { Metabolic yield was based on sugar utilized }\end{array}$ & & \\
\hline
\end{tabular}

The fermentation was complete at 48 hours (Figure 4.3) with a final ethanol concentration of $4.5 \%(\mathrm{w} / \mathrm{v})$ representing a volumetric productivity of $0.92 \mathrm{~g} /(\mathrm{L} \cdot \mathrm{h})$ and ethanol yield of $0.50 \mathrm{~g} / \mathrm{g}$ or $96 \%$ theoretical maximum conversion efficiency for performance with $S$. 
cerevisiae DA2416. The final ethanol concentration 3.5\% (w/v) represented a volumetric productivity of $0.88 \mathrm{~g} /(\mathrm{L} \cdot \mathrm{h})$ and an ethanol yield of $0.48 \mathrm{~g} / \mathrm{g}$ or $94 \%$ theoretical maximum conversion efficiency for performance with Z. mobilis 8b.

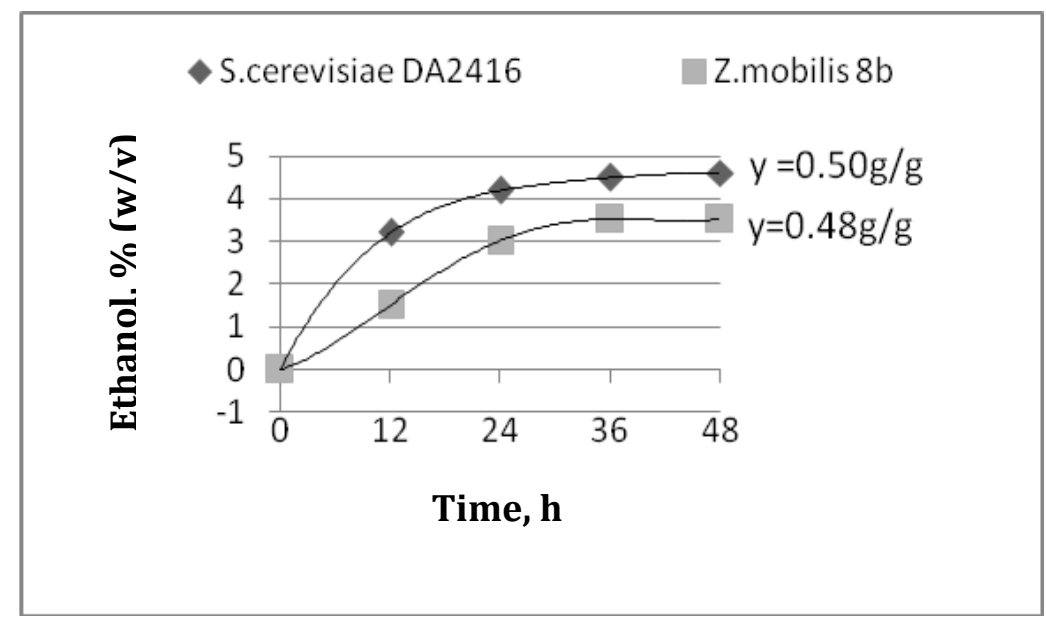

Figure 4.3: Comparative fermentation performance of both strains for ethanol production in time range of 48 hours

In summary, low bacterial activity in fermentation of SSO hydrolysate by Z. mobilis 8b may be attributed to many other factors, including: longer lag phase - an adaptation time for growth condition of chosen strain, low growth rate on SSO hydrolysate and lack of micronutrients such as nitrogen, phosphorus.

\subsection{Conclusions}

The SSO waste samples utilized in this research were pre-processed by the thermal screw press (TSP) and further used as substrates for all enzymatic hydrolysis and fermentation processes. 
COSLIF pretreatments were applied for cellulose extraction from processed source separated organic waste. Results indicated the percent glucan conversion was considerable for COSLIF pretreated samples compared to untreated samples. This study demonstrated and affirmed that S. cerevisiae DA2416 outperformed Z. mobilis 8b on ethanol yields during fermentation process. However, a more comprehensive investigation on lignocellulosic usage with different enzymes and recombinant fermenting strains would be advantageous in biofuel field.

For supporting materials on this chapter please refer to Appendices A "Glucose and ethanol yields calculations” and D “Experimental procedures”. 


\title{
CHAPTER 5. KINETIC MODEL FOR ETHANOL PRODUCTION BY RECOMBINANT S. CEREVISIAE STRAIN ON SOURCE-SEPARATED ORGANIC WASTE
}

\begin{abstract}
An existing kinetic model was adapted and modified to accommodate batch simultaneous saccharification and co-fermentation (SSCF) process on source-separated organic (SSO) waste by the recombinant strain, Saccharomyces cerevisiae DA2416. The model encompasses enzymatic hydrolysis and fermentation processes with competitive uptake of glucose and xylose in both stages. Enzymatic hydrolysis was featured with the addition of a commercially available enzyme, Accellerase 1500, to mediate the process. Pre-processing technologies, including the thermal screw press (TSP) and cellulose-organic-solvent-based lignocellulosic fractionation (COSLIF) pretreatments, were applied on the SSO waste to liberate all fermentable sugars. On average, the sugar yields, mainly in the form of glucose and xylose, from pretreated SSO waste by enzymatic hydrolysis was 90\%. The kinetic model was tailored with experimentally-defined SSO parameters to evaluate the sugar and ethanol yields from SSO waste, and was found to be able predict ethanol production rate accurately with diminutive variance from experiments. Experimental results demonstrated that $S$. cerevisiae DA2416 produced more than $150 \mathrm{~g} / \mathrm{L}$ ethanol, with ethanol yield of $0.50 \mathrm{~g}$ of ethanol/g potential sugar fed, in less than 5 days with 96\% cellulose conversion. It was confirmed in this work that cellulose adsorption capacities along with hydrolysis rate constant have a high impact on sugar and ethanol formation.

This study provides important insights for investigation on the use of SSO waste for ethanol production by S. cerevisiae DA2416 and kinetic model is proven to be a useful tool to help facilitate future process optimization for pilot scale facilities.
\end{abstract}




\subsection{Introduction}

For many years, the main source of fuel for human society has come from fossil resources, which are not infinitive. Lignocellulosic biomass, on the other hand, is a promising alternative to fossil fuels and is considered the only foreseeable sustainable source of organic fuels and materials available to humanity (Shao, 2007). For example, lignocellulosic biomass such as agricultural residues along with the organic fraction of municipal solid waste is particularly attractive because of the low cost and considerable availability, estimated in Ragauskas et al. (2006) and Zhang et al. (2006) at approximately 200 billion metric tons worldwide. As a result, there is an increasing trend for biomass-derived fuel to provide a renewable alternative to conventional fuel for transportation sector. However, the current cost of conversion has been a bottleneck for commercial applications (Houghton et al., 2006). Among the strategies to reduce the processing costs are pretreatment and usage of all fermentable sugars present in biomass with technologies available on the market. Four approaches for cellulosic biomass processing featuring enzymatic hydrolysis have been reported: separate hydrolysis and fermentation (SHF), simultaneous saccharification and fermentation (SSF), simultaneous saccharification and co-fermentation (SSCF) and consolidating bioprocessing (CBP) (Shao, 2007). There are four biologically mediated events identified in each approach, and they are cellulase production, cellulose hydrolysis, pentose fermentation and hexose fermentation (Wyman, 1999; Xiao et al., 2004).

SHF and SSF approaches are featured in many experimental designs for immediate and/or near-term implementation, while SSCF and CBP reqiure more research from deep-rooted problems in the process development (Shao, 2007). Chandrakant and Bisaria (1998) reported a major disadvantage of SHF was inhibition of cellulose hydrolysis by glucose, and as a result it 
has not been possible to obtain glucose concentrations higher than 5.5\% with this method. The SSF approach, featuring enzymatic hydrolysis and fermetation of hexose in one integrated step, considerably increases inhibition to cellulase by cellulose hydrolysis products (Wyman, 1999; Xiao et al., 2004) and is therefore limited in terms of process performance. The SSCF process is similar to SSF except that hexose and pentose fermentations occur in one step. Unlike SHF and SSF, the SSCF process offers potential for more streamlined processing and a lower capital cost (Chandrakant and Bisaria, 1998; Wyman, 1999) . Consequently, the SSCF approach was chosen in this work. SSCF has become more attractive with the emergence of new microorganisms that produce ethanol at a high yield from both glucose and xylose and reduce inhibition hydrolysis by xylose (Kim and Lee, 2005).

The CBP approach has a similar prospective but requires a higher temperature in the range of $50^{\circ} \mathrm{C}$ and $60^{\circ} \mathrm{C}$ for the enzmatic hydrolysis reaction than SSCF. Moreover, studies on CBP with native Clostridium thermocellum was found to produce ethanol with significant amounts of acetic acid, with very limited ability to utilize the xylose from the feed (Demain et al., 2005).

Normally the process design for ethanol production from lignocellulosic biomass is initiated with physiochemical pretreatment to increase the exposure of substrate to enzymatic hydrolysis, followed by the biological conversion of resulting sugars to ethanol by a chosen fermenting strain. A recombinant strain S. cerevisiae DA2416, which is capable of fermenting both glucose and xylose to produce ethanol at high yield, was used in this work. It has been observed that S. cerevisiae DA2416 has by-passed common problems with glucose repression, by taking advantage of an efficient xylose utilization pathway. As a result, the available sugars 
are utilized more effectively, key fermentation parameters, such as overall ethanol yield and inhibition are improved further for better results in the fermentation phase.

A lignocellulosic biomass, made up of pre-processed SSO, which is a blend of approximately $80 \%$ organic "green bin” and approximately $20 \%$ of woodchips from construction/demolition waste, was used in this work as a feedstock for all enzymatic hydrolysis and fermentation processes. Results from previous studies on SSO, including a 6-month compositional analyses (Mirzajani, 2009) and chemical pretreatment (Ehsanipour, 2010), have shown that SSO can become a highly desirable potential substrate with unlimited availability and often at negative cost for future industrial applications.

An existing kinetic model was adapted from ( Zhang, 2008) and modified in this work to predict batch SSCF on SSO waste by glucose and xylose utilizing strain S. cerevisiae DA2416. This model accounts for cellulose and hemicellulose enzymatic hydrolysis and competitive uptake of glucose and xylose. There are only a few published studies on the conversion of cellulose and hemicellulose via SSCF (McMillan et al., 1999; Teixeira et al., 2000; Kim and Lee, 2005) and only one kinetic model has been proposed in the literature (Zhang, 2008). The kinetic model development was based on a semi-mechanic rate equation for cellulose hydrolysis as initially proposed by South et al. (1995) and further modified by Shao (2007) and Zhang (2008) to accommodate cellulose and hemicellulose hydrolysis. The parameters presented in the literature (Shao, 2007; Zhang, 2008) were based on data for paper sludge only. Therefore, new values of interest such as the adsorption capacity, enzymatic hydrolysis constant, ethanol inhibition and ethanol yield were re-established with experimental data to accommodate the batch mode SSCF on SSO waste. 
The main objectives of this paper are: a) to study the influence of different process variables presented in SSCF process configuration on pretreated SSO waste with chosen strain, S. cerevisiae DA2416; b) to develop an experimental kinetic model capable of predicting behavior of batch SSCF on SSO waste with different substrate concentrations: 20 g/L, 50 g/L and $100 \mathrm{~g} / \mathrm{L}$; and c) to compare performance of S. cerevisiae DA2416 on ethanol yield with other yeasts from the same family: S. cerevisiae RWB222 and S. cerevisiae D5A.

The overall objective of this work is to advance in the understanding and gaining knowledge for bioconversion process of converting SSO waste by SSCF approach for ethanol production.

\subsection{Materials and Methods}

The S. cerevisiae DA2416 recombinant strain used in this study was kindly provided by Dr. Yong-Su Jin from the Department of Food Science and Human Nutrition, University of Illinois, USA. It was kept at $-80^{\circ} \mathrm{C}$ in $30 \%(\mathrm{v} / \mathrm{v})$ glycerol for storage. The enzyme complex Accellerase 1500 used in the hydrolysis experiments was donated by of Sigma Aldrich Corp., USA.

The SSO waste utilized in this work was initially pre-processed mechanically, under high temperature $\left(120^{\circ} \mathrm{C}\right)$ and pressure (50 bars) by a thermal screw press for $4-5$ minutes to form a semi-dry stable biomass (Optimum waste and recycling systems Ltd, 2010). SSO samples were prepared as a heterogeneous substrate by blending $20 \% \pm 2 \%$ construction/demolition wood waste in the form of the wood chips and addition 78\%-80\% of organic "green-bin” waste as in (Bekmuradov et al., 2014b). Optimum Waste Recycling Systems, Toronto, Canada, supplied the 
biomass feedstock used in this work (Optimum waste and recycling systems Ltd, 2010). Prior to testing, the SSO waste was oven-dried at $45^{\circ} \mathrm{C}-50^{\circ} \mathrm{C}$ for 48 hours.

Chemical pretreatment with cellulose-organic-solvent-based lignocellulosic fractionation (COSLIF) was applied to release the glucose and xylose from the SSO (Bekmuradov et al., 2014a). Five grams of dry lignocellulose was placed in a 250-mL centrifuge bottle and then mixed with $40 \mathrm{~mL}$ of $85 \%$ concentrated phosphoric acid using a glass rod. The solid/liquid slurry was placed in a benchtop shaking incubator at $150 \mathrm{rpm}$ and $50^{\circ} \mathrm{C} \pm 0.2^{\circ} \mathrm{C}$ for two hours. One hundred $\mathrm{mL}$ of ethanol was then added to the contents and mixed well. After centrifugation at $7000 \mathrm{rpm}$ at room temperature for 15 minutes, the supernatant was decanted. The solid pellet was then re-suspended with $200 \mathrm{~mL}$ of ethanol and centrifuged again at $7000 \mathrm{rpm}$. The supernatant again was decanted. Next, the solid pellet was re-suspended with $200 \mathrm{~mL}$ of distilled water and centrifuged two times and stored in a freezer (Rollin et al., 2009).

Enzymatic hydrolysis experiments were carried out with the addition of a commercially available enzyme, Accellerase 1500. After thawing, the treated solid pellet containing amorphous cellulose was neutralized to $\mathrm{pH} 4.8-5.0$ by $\mathrm{NH} 4 \mathrm{OH}$. The $\mathrm{SSO}$ samples were then brought to $50^{\circ} \mathrm{C}$ before addition of 30FPU/g glucan of Accellerase 1500. Both the $\mathrm{pH}$ value and temperature described were the optimum conditions for the Accellerase 1500 enzyme to mediate hydrolysis to allow release of as much fermentable sugars as possible (Dowe and McMillan, 2008). The hydrolysis experiment was conducted in the shaking incubator (model MAXQ4450). The incubator was set at $250 \mathrm{rpm}$ to keep solids in constant suspension with the temperature of $50^{\circ} \mathrm{C}$ for 72 hours. Samples were taken and measured for sugar content at specified times: 0,12 , 24, 48 and 72 hours. The relevant composition of the SSO was studied and reported in (Bekmuradov et al., 2014a). 
The protein content of the SSO substrate was measured by a modified method of Lowry (Thermo Fisher Scientific Inc., 2011). Adsorption of cellulase onto SSO substrate was done by mixing them in an incubator shaker at $100 \mathrm{rpm}$ in the Innova-40 shaker, at a temperature of $25^{\circ} \mathrm{C}$, in $10-\mathrm{mL}$ glass tubes, under controlled $\mathrm{pH}$ and concentration of cellulase. Centrifugation of the reaction tube followed an incubation period, after which unbound cellulase present in the supernatant were decanted off. The amount of cellulase adsorbed onto a solid substrate was determined as the difference between the total amount of cellulase initially applied $\left[\mathrm{E}_{\text {init }}\right]$ and the amount of free cellulase in the solution [ $\left.E_{\text {non ads }}\right]$. The amount of free cellulase in the solution was measured by rapid UV spectrophotometer technique (Liu et al., 2011; Wang et al., 2012). The technique determines free cellulase concentrations in the solid SSO substrate suspension from the second derivative of the absorption spectra at $750 \mathrm{~nm}$ wavelength through calibration. Each data point in the plots was based on an average of 5 replicates.

The carbohydrate content of SSO was determined by quantitative saccharification (QS) based on 2 hours of incubation in $72 \%$ by weight $\mathrm{H}_{2} \mathrm{SO}_{4}$ at $30^{\circ} \mathrm{C}$ (Ruiz and Ehrman, 1996; Moxley and Zhang, 2007). The cell mass was determined by counting colony forming units on agar plates as described by Zhang et al. (2009).

Following enzymatic hydrolysis, batch soluble sugar fermentation was carried out to evaluate ethanol yields from SSO samples as a result of conversion using recombinant strain $S$. cerevisiae DA2416. Soluble sugar batch fermentation was performed in 250-mL serum bottles with $100 \mathrm{~mL}$ working volume. Temperature was maintained at $30^{\circ} \mathrm{C}$ and $\mathrm{pH}$ was controlled at 6.0 by $1 \mathrm{M}$ potassium hydroxide $(\mathrm{KOH})$ as suggested by previous study (Mohagheghi et al., 2004). Compositional analysis of the samples for ethanol concentrations was carried out at 0,12 , 24 and 48 hours) by HPLC (see Appendix E “Samples of HPLC runs for glucose and ethanol”). 
The kinetic model adapted in this study uses a semi-mechanistic rate equation for cellulose hydrolysis as proposed by South et al. (1995) and further modified in Zhang et al. (2009). The parameters presented in Zhang's kinetic model were based on cellulose and hemicellulose hydrolysis for pretreated paper sludge. In this study adsorption parameters were re-established based on overall carbohydrate content of pretreated SSO waste. The binding capacity or specific capacity of the carbohydrate component for cellulase of SSO samples was obtained using Langmuir isotherms. The remaining cellulose hydrolysis parameters were as reported by Zhang (2008) . Experimental data on glucose and xylose consumptions and growth parameters were fitted using the non-linear function of Polymath 5.1 (Polymath Software: Willimantic, CT, USA). All other parameters were dynamically fitted with the curve fitting function in the Berkeley Madonna computer program with a fourth-order Runge-Kutta algorithm. Runs were performed on a standard laptop.

A sensitivity analysis was carried out based on the least-square method (Stigler, 1986; Bretscher, 1995). It was performed to test the impact of the value of important parameters on the model prediction of ethanol production if the values were changed to $\pm 10 \%$ from those obtained from experiments. The analysis was performed to determine the difference between experimental data and modified kinetic model predictive ability.

\subsection{Results and Discussion}

Due to its prospect for commercial application, SSO waste was chosen as the substrate to evaluate the values on sugar and ethanol yields by fermentation using S. cerevisiae DA2416 strain. 
Approximately, more than half of the original sample was composed of moisture. Essential polymeric sugars in the oven dried SSO samples included: 31\% glucose, 19\% xylose, and about $9 \%$ of other sugars and $23 \%$ of lignin. These homogeneous samples had a $\mathrm{pH}$ range of 5.2-5.5. The SSO samples pretreated by concentrated phosphoric acid ( $85 \% \mathrm{w} / \mathrm{w})$ and ethanol ( $95 \% \mathrm{v} / \mathrm{v})$ were hydrolyzed and glucan digestibility was found to be $72 \%$ after 24 hours and $90 \%$ after 72 hours. The high glucan digestibility was achieved for the COSLIF-pretreated SSO with addition of $30 \mathrm{FPU} / \mathrm{g}$ glucan of Accellerase 1500 (Bekmuradov et al., 2014b).

Recognizing that cellulase mixtures contain a mixture of cellulase and hemicellulose portions which bind to cellulose and hemicellulose, adsorption capacity constant " $\sigma$ " was recalculated using the modified method of Lowry and resultantly they were in the range of 0.264 to 0.280 . Similarly, the binding capacity $\mathrm{C}_{\mathrm{S}}$ was determined as between $0.442 \mathrm{~g}$ protein/g carbohydrate and 0.466 g protein/g carbohydrate using Langmuir isotherms. Cellulose hydrolysis rate constant $-\mathrm{k}(1 / \mathrm{h})$ had range values of 0.662 and 0.725 . The remaining cellulose enzymatic hydrolysis parameters were adapted from Zhang (2008). Adsorption of Accellerase 1500 cellulase to the SSO waste samples was evaluated after hydrolysis was allowed to proceed for specified time, $(6,12,24,36,48,60$ and 72 hours) resulting in various values for fractional conversion up to $85 \%$ as shown in Figure 5.1 . 


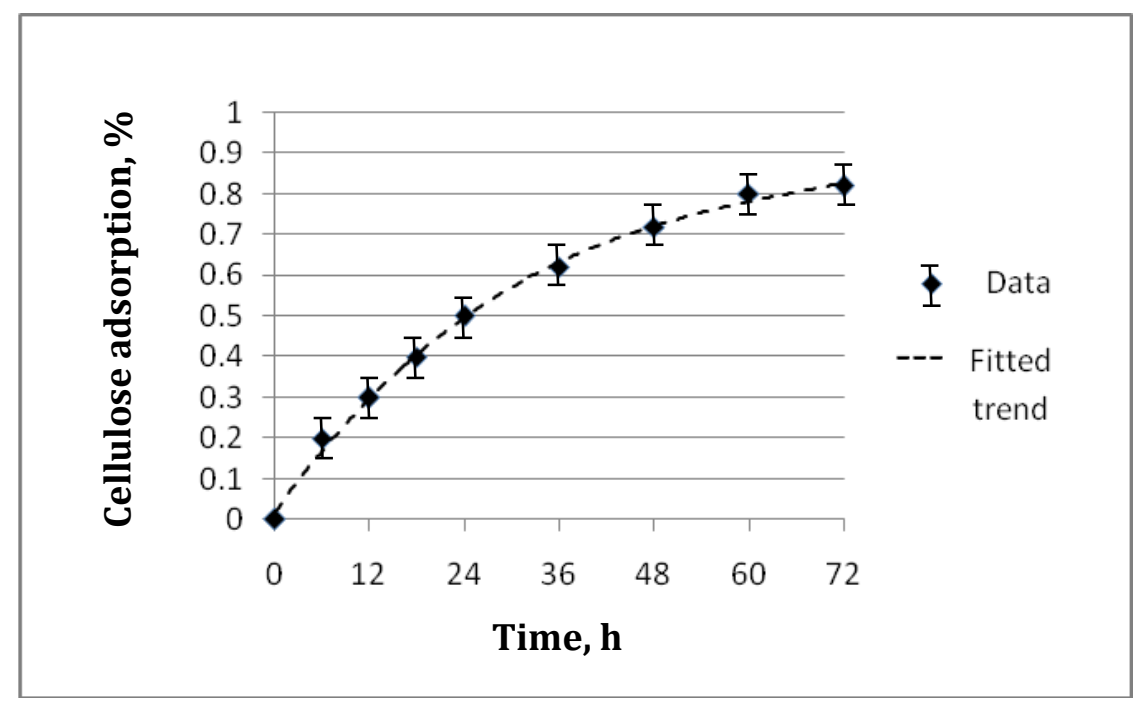

Figure 5.1: Cellulose adsorption data for pretreated SSO samples

The predictions were confirmed by experimental data obtained during the addition of Accellerase 1500 enzyme complex to SSO samples throughout the course of hydrolysis. The predictions over the time of reaction are almost the same. The good fit obtained in this study suggests that as for the SSO samples it seems reasonable to assume a constant adsorption capacity normalized to the amount of cellulose remaining and there is no reason to hypothesize adsorption affinity as a function of a conversion.

Adsorption parameters $K_{S}$ and $\sigma_{S}$ in Table 5.1 were then fit to the data of all conversions by minimizing the sum of squares for the predicted and experimental data. The new adsorption parameters with conversion data were used to fit the parameters $k, c$ and $e$ in the cellulose rate equation by South et al. (1995). The values of parameters are presented in Table 5.1. 
Table 5.1: Average parameter values for SSCF of the SSO.

\begin{tabular}{lll}
\hline $\mathrm{K}_{\mathrm{S}}$ & 0.466 & This work \\
$\sigma_{\mathrm{S}}$ & 0.280 & This work \\
$\mathrm{k}$ & 0.725 & This work \\
$\mathrm{e}$ & 0.516 & This work \\
$\mathrm{c}$ & 0 & Shao, 2007 \\
\hline
\end{tabular}

With parameter values in hand for adsorption, hydrolysis and fermentation, a pre-existing kinetic model for SSCF was modified to account newly defined SSO feedstock constants. In view of this, we selected a simple correlation model reported by Zhang (2008) as in the following equation:

$$
r_{X n}=\frac{X I}{G I} \times r_{G n}
$$

where $r_{G n}$ and $r_{X n}$ - formation of glucan and xylan respectively, GI, XI - initial glucan and xylan concentrations.

Equation (1) above describes the correlation of glucan and xylan hydrolysis and is derived from the simple relationship of $\mathrm{X}_{1}=\mathrm{X}_{2}$ in which: $\mathrm{X}_{1}$ and $\mathrm{X}_{2}$ are the conversion of glucan and xylan respectively. To understand the enzyme hydrolysis performance with the S. cerevisiae DA2416 strain, the percentage of glucan and xylan conversion was calculated. Glucan and xylan used in SSO in this work were converted to monomeric sugars at almost the same rate by Accellerase 1500 . The average glucan conversion to monomer sugars was $96 \%$, and the average xylan conversion to monomer sugars was $94 \%$ at $30^{\circ} \mathrm{C}$. However, we observed a slightly higher 
residual xylose accumulation than residual accumulation of glucose during experimental tests. The slower consumption rate of xylose than glucose was consistent with the fermentation of soluble sugars in other studies as well utilizing S. cerevisiae (Kuyper et al., 2005; Zhang and Lynd, 2010).

To begin SSCF runs, Accellerase 1500 enzyme at 30FPU cellulase were mixed with yeast innocula and added into the vessel to increase the sugar's accessibility to cells during the mass transfer limited period. High glucan digestibility (approximately 90\%) was achieved. Batch soluble sugar fermentation experiments were carried out to find the fermentation related constants, exclusive ethanol inhibition and yield in SSCF kinetic model by performance of recombinant strain S. cerevisiae DA2416. In S. cerevisiae strains, there are a large number of genes encoding hexose transporters (Reifenberger, 1997), which are also believed to function with low affinity xylose transporters in recombinant xylose utilizing S. cerevisiae strains (Sedlak and Ho, 2004). Based on this examination, a competitive substrate inhibition model for growth in glucose and xylose by S. cerevisiae DA2416 was chosen to capture the growth kinetics. Inhibition of growth and fermentation has been described using different equations in the literature, including exponential inhibition, linear inhibition, and linear inhibition beyond threshold (van Uden, 1989). Among them, a threshold linear inhibition model was chosen because it fit best with the data. A threshold linear inhibition model equation (2) as described elsewhere (South et al., 1995) accounts for glucose fermentation with an additional term representing sugar uptake from xylose and inhibition from ethanol:

$$
\mu_{\mathrm{Gl}}=\left[\frac{\mathrm{X} \cdot \mu_{\mathrm{Gl}}^{\mathrm{Max}} \cdot \mathrm{G}_{\mathrm{l}}}{\mathrm{K}_{\mathrm{Gl}}+\mathrm{G}_{\mathrm{l}}+\mathrm{I}_{1} \cdot \mathrm{X}_{\mathrm{l}}}\right] \times\left(1-\frac{\mathrm{Eth}}{\mathrm{Eth}_{\mathrm{Gl}}^{\mathrm{Max}}}\right)^{\mathrm{f} 1}
$$


where $\mu_{G l}^{M a x}$ and $E t h_{G l}^{M a x}$ - maximum specific growth rate and maximum ethanol concentration for growth on glucose respectively; $G_{l}, X_{l}, E t h$ - concentration of glucose, xylose and ethanol; $K_{G l}, I_{1}, f^{1}$ - related constants.

The rate of formation of xylose was described by South et al. (1995), similar to that used for glucose formation shown in the following equation:

$$
\mu_{X l}=\left[\frac{X \cdot \mu_{X l}^{M a x} \cdot\left(X_{l}-X_{l T}\right.}{K_{X l} \cdot X+X_{l}+I_{2} \cdot G_{l}}\right] \times\left(1-\frac{E t h}{E t h_{X l}^{M a x}}\right)
$$

where $\mu_{X l}^{\operatorname{Max}}, E_{h_{X l}}^{\operatorname{Max}}$ - maximum specific growth rate and maximum ethanol concentration for growth on xylose respectively; $K_{X l}, I_{2}$ - related constants; $X_{l T}$ - threshold concentration.

The well-known phenomenon of declining hydrolysis rate (Zhang, 2009) as the reaction progresses was modeled using the following empirical equation:

$$
k(x)=k[1-x(t)]^{e}+c
$$

Values for inhibition factors $I_{1}$ and $I_{2}$ in this work were found to be 0.108 and 6.032 respectively, indicating that the inhibition of xylose utilization by glucose is more than 50 times stronger than the inhibition of glucose utilization by xylose.

In order to test the SSCF performance on SSO feedstock with newly redefined constants, batch fermentations were carried out in a separate series of experimental evaluation at different substrate concentrations of $20 \mathrm{~g} / \mathrm{L}, 50 \mathrm{~g} / \mathrm{L}$ and $100 \mathrm{~g} / \mathrm{L}$ with the enzyme loading of 30FPU cellulase. Initial substrate concentration for model calibration is kept low, below $10 \mathrm{~g} / \mathrm{L}$, so, that 


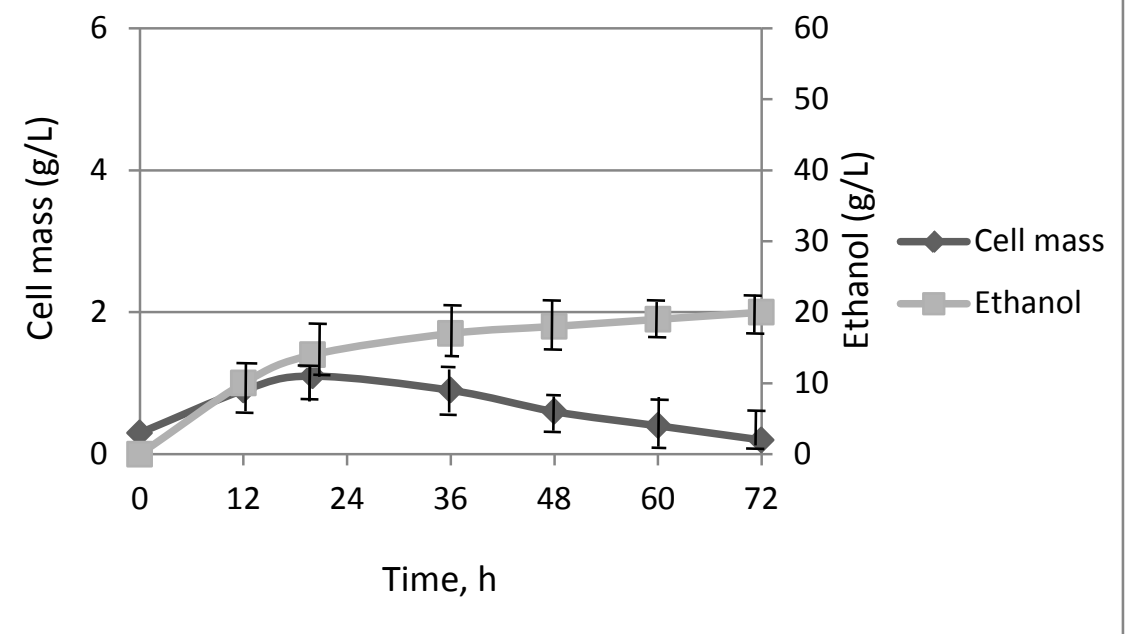

-Cell mass-expt _ Cell mass-model

=thanol-expt Ethanol-model

Figure 5.2: Experimental data and kinetic model prediction for SSO samples (substrate concentration $20 \mathrm{~g} / \mathrm{L})$.

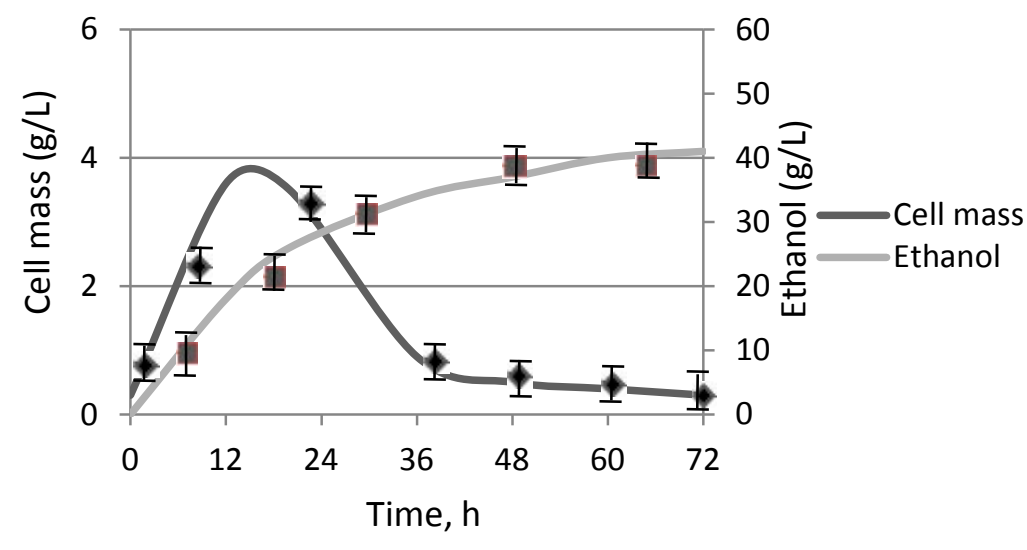

- Cell mass-expt —Cell mass-model

= Ethanol-expt -Ethanol-model

Figure 5.3: Experimental data and kinetic model prediction for SSO samples (substrate concentration $50 \mathrm{~g} / \mathrm{L}$ ) 


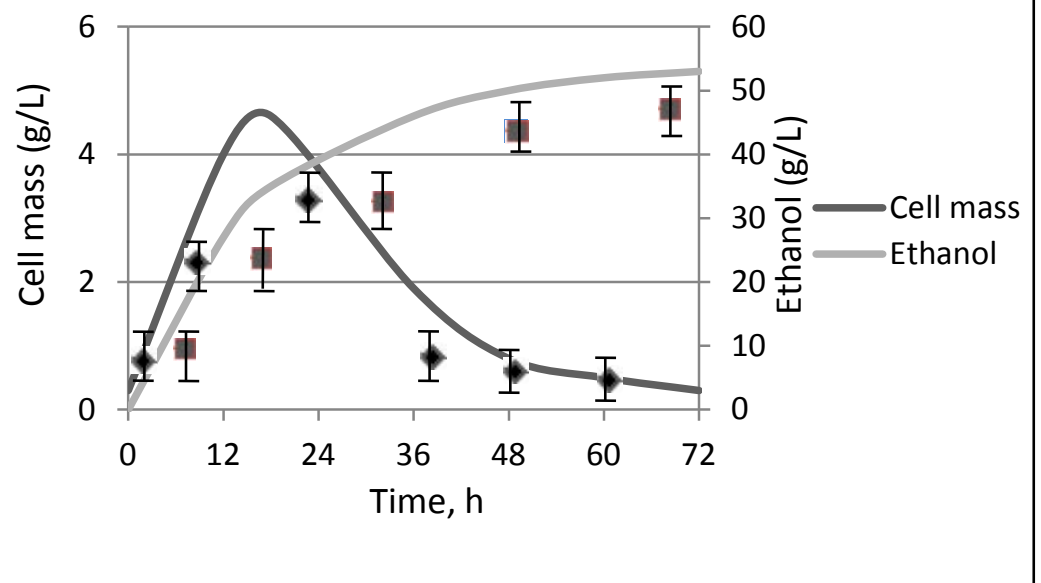

- Cell mass-expt _ C Cell mass-model

= Ethanol-expt _Ethanol-model

Figure 5.4: Experimental data and kinetic model prediction for SSO samples (substrate concentration $100 \mathrm{~g} / \mathrm{L}$ )

effect of produced ethanol can be ignored. The model accurately predicts the sugar and ethanol concentration along with cell mass for $20 \mathrm{~g} / \mathrm{L}$ and $50 \mathrm{~g} / \mathrm{L}$ (experimental data shown with error margins) as shown in Figures 5.2, 5-3, but not for $100 \mathrm{~g} / \mathrm{L}$ (Figure 5.4).

In higher substrate concentration mode, reaction deviated from experimental values as shown in Figure 5. 4, suggesting ethanol inhibition is a factor causing this discrepancy. There are also other reasons attributed to it, for example, enzyme deactivation or enzyme adsorption by lignin and/or obstruction of lignin on the surface of cellulose to the point that enzymes are not able to access cellulose (Collins, 2007; Faye, 2010).

Another set of experiments was conducted to assess the contribution of ethanol inhibition to the loss of cell viability. We expected good cell viability at lower SSO sample concentrations if inhibition was a major factor for cells lost. It was noticed in experiments that ethanol 
concentration was a major factor of declining cell viability, but at the same time, one or more factors other than ethanol inhibition that were not yet determined might contribute to the loss viability in SSCF using S. cerevisiae DA2416. Table 5.2 below presents the results of sensitivity analysis.

In the sensitivity analysis, the sum of variance between the measured ethanol and the ethanol predicted by the model were calculated. Single parameters were varied to $\pm 10 \%$ of the experimental values and responses $S^{*}$ were calculated as absolute values based on the leastsquare method (Stigler, 1986; Bretscher, 1995), where $S^{*}$ is defined as in the following equation:

$$
S^{*}=\left|\frac{V_{2}-V_{1}}{V_{1}}\right|
$$

where $\mathrm{V}_{2}$ - sum of variance between measured and predicted values from kinetic modelwith a single model constant changed to $\pm 10 \%$; $\mathrm{V}_{1}$ - sum of variance between the measured and predicted value with all kinetic model constants unchanged from their measured values.

As seen from Table 5.2, cellulase adsorption constant $C_{S}$ had the highest response value followed by the cellulose enzymatic hydrolysis constant $k$, among the enzymatic hydrolysis constants. From the results, a $10 \%$ decrease of $C_{S}$ is almost 3 times more sensitive than a $10 \%$ increase, demonstrating that increase in enzyme loading will be more effective than increase in substrate concentration for ethanol yield. Among the microbial growth related constants, the ethanol yield from glucose and xylose exhibited the highest sensitivity, with ethanol tolerant related constants showing moderate sensitivity.

Finally, a comparison of the performance of S. cerevisiae DA2416 with different $S$. cerevisiae strains in terms of the ethanol production and glucan and xylan conversions are performed and the results are presented in Table 5.3. The results in Table 5.3 shows the highest 
Table 5.2: Sensitivity analysis of kinetic parameters for SSO used in this work

\begin{tabular}{|c|c|c|c|c|c|}
\hline Parameter & Value & Unit & Source & $\begin{array}{c}\mathrm{S}^{*} \text { value } \\
(10 \%) \\
\text { increase }\end{array}$ & $\begin{array}{c}\mathrm{S}^{*} \text { value } \\
(10 \%) \\
\text { Decrease }\end{array}$ \\
\hline
\end{tabular}

Enzymatic hydrolysis constants

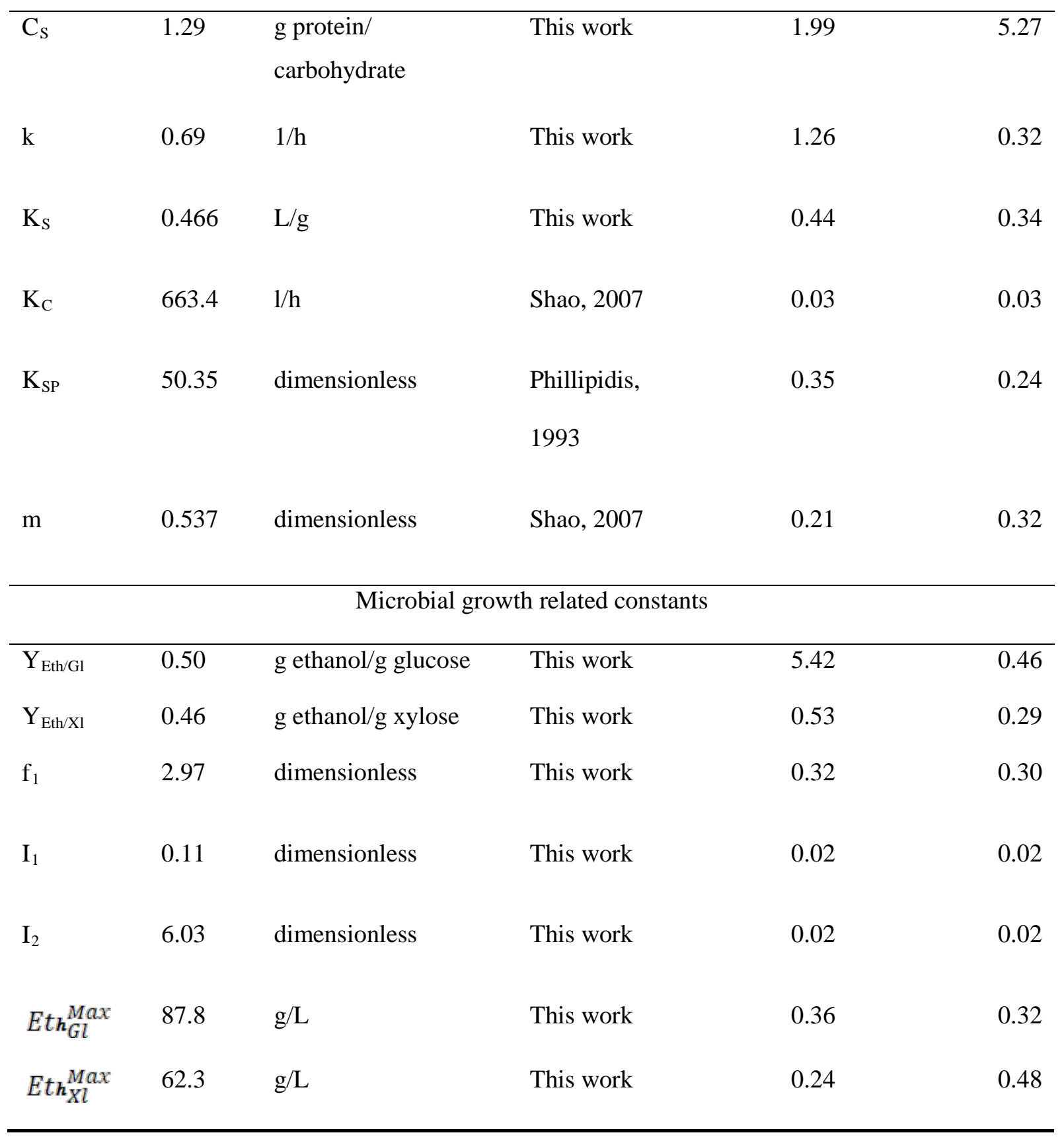

$\mathrm{S}^{*}$ - sensitivity factor, represents the response to a given $10 \%$ increase or decrease of single constant value 
ethanol production achieved for S. cerevisiae DA2416 by SSCF on SSO, as compared to those by S. cerevisiae RWB222 and S. cerevisiae D5A as tested on paper sludge. Experimental results demonstrated S. cerevisiae DA2416 produced an ethanol yield of $0.50 \mathrm{~g}$ of ethanol/g potential sugar fed on SSO in less than 5 days with 96\% cellulose conversion totalling in $150 \mathrm{~g} / \mathrm{L}$ ethanol.

All strains exhibited almost the same value of glucan and xylan conversion. Performance of S. cerevisiae DA2416 on SSO has a higher ethanol production, most probably due to a higher enzymatic activity and higher tolerance to inhibitors than other two strains. Although, different substrates, including paper sludge should be tested to validate the DA2416 strain in the future work.

Table 5.3: Comparison performance of different S.cerevisiae strains

\begin{tabular}{lccc}
\hline S. cerevisiae strain: & DA2416 & RWB222 & D5A \\
\hline g ethanol/g sugar consumed & $0.48 \pm 0.01$ & $0.40 \pm 0.01$ & $0.44 \pm 0.01$ \\
g ethanol/g sugars fed & $0.50 \pm 0.01$ & $0.35 \pm 0.01$ & $0.33 \pm 0.01$ \\
final glucan conversion & $0.96 \pm 0.01$ & $0.89 \pm 0.02$ & $0.94 \pm 0.01$ \\
final xylan conversion & $0.94 \pm 0.02$ & $0.88 \pm 0.02$ & $0.93 \pm 0.02$ \\
\% ethanol production compare to D5A & 152 & 105 & 100 \\
\hline
\end{tabular}

S. cerevisiae DA2416@30 $\mathrm{C}$ - this work

S. cerevisiae RWB222 @ 30 ${ }^{\circ} \mathrm{C}$ - (Zhang and Lynd, 2010)

S. cerevisiae D5A @ 37 C- (Zhang and Lynd, 2010) 


\subsection{Conclusions}

The SSO waste samples utilized in this research were pre-processed by the TSP and further used as substrates for all COSLIF, enzymatic hydrolysis, and fermentation processes.

COSLIF pretreatment was applied for cellulose and sugars extraction from pre-processed SSO waste. Fermentation results demonstrated S. cerevisiae DA2416 produced more than 150 $\mathrm{g} / \mathrm{L}$ ethanol with ethanol yield of $0.50 \mathrm{~g}$ of ethanol/g potential sugar fed on SSO in less than 5 days. It was demonstrated that a kinetic model with integrated values of experimentally defined SSO feedstock constants was successful in predicting the ethanol yield accurately with diminutive variance from experiments. The cellulose adsorption constant, ethanol tolerance, and ethanol yield played very important roles in the fermentation process. It was identified in this work as confirmed fact that glucose and xylose utilization were inhibited by each other to a certain extent or region, specifically in the region with low sugar concentration level. Typically, ethanol production rate in SSCF with low sugar concentrations is highly depends on enzymatic hydrolysis rate. As a consequence, at substrate concentration levels (e.g. between 20 g/L up to 50 $\mathrm{g} / \mathrm{L}$ ), the rate predicted by kinetic model was in good agreement with experimental data and showed the sign of deviation at concentration rate of $100 \mathrm{~g} / \mathrm{L}$, suggesting that ethanol inhibition was a main cause of discrepancy. Therefore, the discrepancy between experiments and kinetic model predictions, particularly at high substrate concentrations, needs to be examined more comprehensively.

Additionally, a good fermenting strain should have the ability to withstand ethanol toxicity and common inhibitors such as aliphatic acids, furan aldehydes, furfural and inorganic compounds. 
This study demonstrated and affirmed that S. cerevisiae DA2416 is a promising strain for SSO substrate in SSCF. In the future, the kinetic model used should be expanded to introduce the inference of lignin in lignocellulosic biomass.

For supporting materials on this chapter please refer to Appendices B “Absorption capacity calculations” and C “Berkeley Madonna computer program code and tabulated data”. 


\section{CHAPTER 6. CONCLUSIONS AND FUTURE WORK RECOMMENDATIONS}

\subsection{Conclusions}

This thesis sought to gain a better understanding of bioconversion process of pretreated SSO waste by SHF and SSCF process configurations via experimental work and kinetic modeling. It was found in this study that: a) SSO is an excellent feedstock material for ethanol conversion with total fermentable sugars available in the samples for conversion in the range of $40 \%-41 \%$, however, high lignin content in the samples, $27 \%-30 \%$, emphasize on the need for pretreatment; b) modified COSLIF method is proven to be a viable method for pretreatment of SSO feedstock with the efficiency improved by $20 \%$ using ethanol instead of acetone. On average, glucose yield from SSO samples pretreated by modified COSLIF was approximately $90 \%$ versus $70 \%$ by standard method after enzymatic hydrolysis; c) S. cerevisiae DA2416 is a promising strain for SSO substrate in SSCF runs with ethanol yield of $0.50 \mathrm{~g}$ of ethanol/g sugar fed or $96 \%$ substrate conversion ; and d) kinetic model with newly interpolated values of experimentally defined SSO feedstock constants is able to predict the batch fermentation process well at substrate concentration range between $20 \mathrm{~g} / \mathrm{L}$ and $50 \mathrm{~g} / \mathrm{L}$, but noticeably deviated at substrate concentration of $100 \mathrm{~g} / \mathrm{L}$.

The major parts of this study include: a) experimental work of biochemical pretreatment of SSO waste as a valuable source of cellulosic biomass for ethanol production only; b) comparative study of ethanol production from pretreated by modified COSLIF method on SSO samples by two different microorganisms, bacteria - Z. mobilis 8b and recombinant yeast - S. cerevisiae DA2416; and c) alteration of existing kinetic model with newly experimentally 
defined SSO feedstock constants to predict batch SSCF on SSO waste with different substrate concentrations. The findings of this thesis are described below.

\subsection{Experimental Work}

The experimental work for this thesis consisted of three categories: experiments for SSO pretreatments, which include thermal screw press followed by COSLIF, enzymatic hydrolysis, fermentation, and kinetic modeling.

"Pre-processing” technologies, including the TSP and COSLIF were used with respect to pretreatments. An improvement on the standard method of COSLIF pretreatment was developed based on lower enzyme loading and using an ethanol washing instead of acetone. Results indicated the percent glucose conversion was considerable (90\% versus $70 \%$ ) for the modified COSLIF method with a significant glucose yield approximately $90 \%$.

High glucan digestibility of the pretreated SSO was accredited to drastic changes in the supramolecular structure of the biomass before and after the COSLIF pretreatment, observed by the SEM in this study (Figure 3.5; Chapter 3).

The glucose digestibility of the pretreated standard COSLIF sample was approximately $70 \%$ as presented in (Figure 3.3; Chapter 3) with high enzyme loading of 60FPU and acetone washing. On the other hand, with a lower enzyme loading, 30FPU and ethanol washing, it reached $90 \%$ digestibility after 36 hours. This suggests that by removing hemicelluloses and lignin barriers, there was an increase in accessibility to the cellulose change by the cellulobiose, while also reducing the competitive inhibition of xylan to endo-glucanase. The effectiveness of the enzymatic hydrolysis was gauged by assessing the potential inhibitory factors and effects of 
fermentation. The high ethanol yield presented in (Figure 4.3; Chapter 4) indicated very little inhibitors were present in the hydrolysates that were pretreated by the modified COSLIF method.

In cases of kinetic modeling, experiments were performed to obtain new data (cellulose adsorption constant, enzymatic hydrolysis rate, ethanol yields from glucose and xylose, and ethanol tolerance) for comparison with model predictions. The good agreement between data and kinetic model fit indicated that model was robust and accurately predicted the sugar and ethanol concentration along with cell mass for $20 \mathrm{~g} / \mathrm{L}$ and $50 \mathrm{~g} / \mathrm{L}$ (Figures 5.2 and 5.3; Chapter 5), but not for $100 \mathrm{~g} / \mathrm{L}$. In higher substrate concentration mode, reaction deviated from experimental values as shown in (Figure 5.4; Chapter 5) suggesting that there were other reasons attributed to it, e.g. enzyme adsorption by lignin and/or obstruction of lignin on the surface of cellulose to that point when enzyme were not able to access cellulose.

\subsection{SSO Waste as a Lignocellulosic Feedstock for Ethanol Production}

Big metropolitan cities around the world have been experiencing a challenge over the past few years regarding their waste problems, and having inadequate infrastructure for effective waste management practices. The city of Toronto in Canada is a perfect example with its waste problems that have recently become more critical. With the anticipation of landfills' closure and environmental concerns, the city of Toronto requires more efforts in developing economically feasible strategies for dealing with its waste. One of these strategies is using organic fraction of municipal waste such as SSO, which has a great potential for production of value added fuels and chemicals. 
The experimental results showed a relatively high amount of carbohydrates in the SSO samples (glucose - 31\% and xylose - 19\%), indicating a great potential of SSO to be utilized as an ethanol production feedstock instead of using other cellulosic feedstocks such as herbaceous energy crops. A technology for the biological conversion of SSO to ethanol was proposed based on the current techniques and the results from the characterization study on SSO. It included physical pretreatment by TSP machine, chemical by COSLIF, enzymatic hydrolysis with addition of enzyme complex Accelerase 1500 and fermentation by two recombinant strains: $Z$. mobilis 8b and S. cerevisiae DA2416.

\subsection{Comparative Ethanol Productivities of two Different Recombinant Fermenting Strains on} the SSO Waste

The SSO waste samples utilized in this research were pre-processed by the thermal screw press and further used as substrates for all enzymatic hydrolysis and fermentation processes. COSLIF pretreatments were applied for cellulose extraction from processed source separated organic waste. Results indicated the percent glucan conversion was considerable for COSLIF pretreated samples compared to untreated samples (Figure 4.1; Chapter 4). This study demonstrated that S. cerevisiae DA2416 outperformed Z. mobilis 8b on ethanol yields during fermentation process. At 48 hours of fermentation, ethanol yield was equivalent to $0.48 \mathrm{~g}$ of ethanol produced per gram of SSO biomass by Z. mobilis $8 \mathrm{~b}$ and $0.50 \mathrm{~g}$ of ethanol produced per gram of SSO biomass by S. cerevisiae DA2416. However, a more comprehensive investigation on lignocellulosic usage with different enzymes and recombinant fermenting strains would be advantageous in bio-fuel field. 


\subsection{Kinetic Modeling}

A kinetic model adapted and altered in this work to predict batch SSCF on SSO waste by new recombinant strain S. cerevisiae DA2416. To the knowledge of the author, this is first kinetic model, which was specifically applied for SSO feedstock. The kinetic model included adapted correlation of cellulose and xylan enzymatic hydrolysis, competitive substrate uptake, xylose utilization kinetics, and accelerated cell death due to ethanol exhibition. The most important parameters for enzymatic hydrolysis (adsorption capacity and enzymatic hydrolysis constant) and for fermentation (ethanol yield and maximum ethanol tolerance) were measured or re-calculated to address new changes for SSO feedstock. The newly re-developed kinetic model accurately predicted the sugar and ethanol concentrations along with cell mass for $20 \mathrm{~g} / \mathrm{L}$ and 50 g/L (Figures 5.2 and 5.3), but not for $100 \mathrm{~g} / \mathrm{L}$ (Figure 5.4). There were a several causes for that: enzymes deactivation; high concentration of inhibitors presented in SSO; and high concentration of ethanol in the tested broth.

\subsection{Future Work Recommendations.}

Two lines of further study are recommended:

1) More research should be done with different enzyme complexes to reduce or avoid byproducts production such as ethyl, methane, and acetic acid. As for Accelerase 1500 enzyme complex, it is unknown wither it attaches to hemicelluloses accurately. An enzyme complex with a higher ethanol resistance should be sought.

2) The kinetic model re-developed in this work can predicts the sugar and ethanol concentrations from SSO on SSCF by S. cerevisiae DA2416 for the low substrate 
concentration mode. The discrepancy between kinetic model prediction and experimental data at higher substrate concentration needs to be examined further. Besides the limitation at high substrate concentration, future kinetic models should address the interference of lignin for its application as well as an introduction of other feed stocks pretreated by different approaches. Other real world substrates should be tested by different $S$. cerevisiae fermenting microorganisms. 


\section{APPENDIX A: GLUCOSE AND ETHANOL YIELDS CALCULATIONS}

Glucose yield from SSO by Quantitative Saccharification (QS)

Date: Aug 7, 2013

Table 1: Moisture Content Measurement

\begin{tabular}{cccccccc}
\hline Sample & $\begin{array}{c}\text { Mass of } \\
\text { Foil } \\
(\mathrm{g})\end{array}$ & $\begin{array}{c}\text { Mass of } \\
\text { Foil }+\end{array}$ & $\begin{array}{c}\text { Mass of } \\
\text { Sample } \\
(\mathrm{g})\end{array}$ & $\begin{array}{c}\text { Mass of } \\
(\mathrm{g})\end{array}$ & $\begin{array}{c}\text { Mass of } \\
\text { Dried Foil } \\
+ \text { Sample } \\
\text { Dried }\end{array}$ & $\begin{array}{c}\text { Total } \\
\text { Sample } \\
\text { Solid } \\
(\%)\end{array}$ & $\begin{array}{c}\text { Moisture } \\
\text { Contents } \\
(\%)\end{array}$ \\
\hline 1 & 1.330 & 3.330 & 2.000 & 2.450 & 1.120 & 56.00 & 44.00 \\
2 & 1.320 & 3.320 & 2.000 & 2.510 & 1.190 & 59.50 & 40.50 \\
3 & 1.320 & 3.320 & 2.000 & 2.270 & 0.950 & 47.50 & 52.50 \\
4 & 1.320 & 3.320 & 2.000 & 2.360 & 1.040 & 52.00 & 48.00 \\
5 & 1.320 & 3.320 & 2.000 & 2.370 & 1.050 & 52.50 & 47.50 \\
Avg & & & & & & 53.50 & 46.50 \\
\hline
\end{tabular}




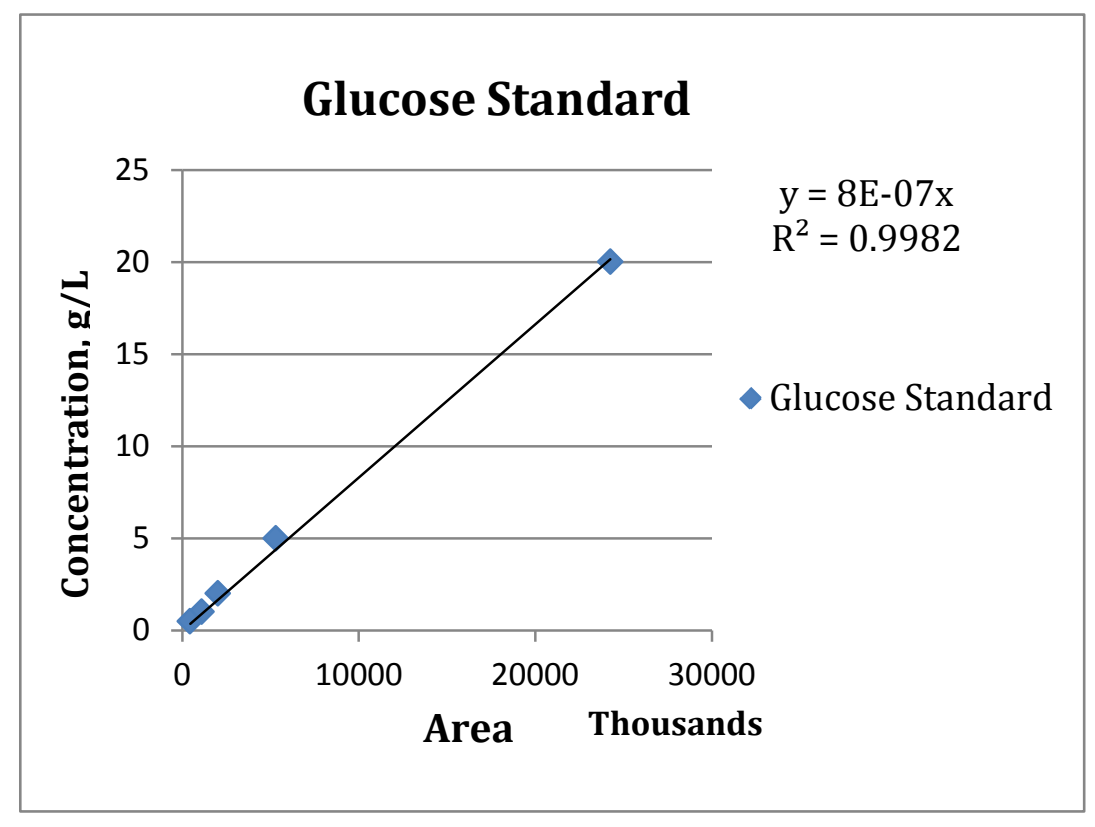

Figure 1: Glucose standards plot

Table 2: Glucose responses vs different concentrations

\begin{tabular}{ccc}
\hline Standards & Conc. g/L & \multicolumn{1}{c}{$\begin{array}{c}\text { Glucose } \\
\text { Area }(\mu \mathrm{V} . \mathrm{S})\end{array}$} \\
\hline S1 & 20 & 24250902.38 \\
S2 & 5 & 5311241.79 \\
S3 & 2 & 2020925.80 \\
S4 & 1 & 1096904.40 \\
S5 & 0.5 & 449967.00 \\
\hline
\end{tabular}


Table 3: Glucose yield calculations

\begin{tabular}{|c|c|c|c|c|c|c|c|c|c|c|c|}
\hline \multirow[t]{2}{*}{$\begin{array}{c}\text { Bottle } \\
\#\end{array}$} & $\begin{array}{c}\text { Sample } \\
\text { Name }\end{array}$ & $\begin{array}{l}\text { Glucose } \\
\text { (HPLC) }\end{array}$ & $\begin{array}{l}\text { Glucose } \\
\text { (HPLC) }\end{array}$ & $\begin{array}{l}\text { Glucose } \\
\text { (HPLC) }\end{array}$ & $\begin{array}{l}\text { Corrected } \\
\text { Glucose }\end{array}$ & $\begin{array}{c}\text { Glucose } \\
\text { in } 0.3 \mathrm{~g} \\
\text { QS } \\
\text { Sample }\end{array}$ & $\begin{array}{c}\text { Pretreated } \\
\text { SSO/ } \\
\text { Bottle } \\
\text { (Avg) }\end{array}$ & $\begin{array}{c}\text { Pretreated } \\
\text { SSO/ } \\
\text { Bottle } \\
\text { w/o } \\
\text { Moisture }\end{array}$ & $\begin{array}{l}\text { Glucose } \\
\text { per } \\
\text { Pretreated } \\
\text { Dry SSO } \\
\text { Bottle }\end{array}$ & $\begin{array}{c}\text { Glucan } \\
\text { per } \\
\text { Pretreated } \\
\text { Dry SSO } \\
\text { Bottle }\end{array}$ & $\begin{array}{c}\text { Glucan } \\
\text { Yield } \\
\text { per } 5 \text { g } \\
\text { SSO } \\
\text { Bottle }\end{array}$ \\
\hline & & Area( $\mu$ V.S) & $\begin{array}{l}\text { Con. } \\
\text { (g/L) }\end{array}$ & $\begin{array}{l}\text { Con. } \\
\text { (g/L) }\end{array}$ & (g/L) & (g) & (g) & (g) & (g) & (g) & (\%) \\
\hline \multirow{2}{*}{1} & QS1 & 609238.46 & 0.49 & \multirow{2}{*}{0.44} & \multirow{2}{*}{0.65} & \multirow{2}{*}{0.056} & \multirow{2}{*}{5.000} & \multirow{2}{*}{2.675} & \multirow{2}{*}{0.503} & \multirow{2}{*}{0.453} & \multirow{2}{*}{16.9} \\
\hline & QS2 & 482873.30 & 0.39 & & & & & & & & \\
\hline \multirow{2}{*}{2} & QS3 & 391310.31 & 0.31 & \multirow{2}{*}{0.30} & \multirow{2}{*}{0.45} & \multirow{2}{*}{0.039} & \multirow{2}{*}{5.000} & \multirow{2}{*}{2.675} & \multirow{2}{*}{0.348} & \multirow{2}{*}{0.313} & \multirow{2}{*}{11.7} \\
\hline & QS4 & 364344.20 & 0.29 & & & & & & & & \\
\hline \multirow{2}{*}{3} & QS5 & 418668.64 & 0.33 & \multirow{2}{*}{0.32} & \multirow{2}{*}{0.47} & \multirow{2}{*}{0.041} & \multirow{2}{*}{5.000} & \multirow{2}{*}{2.675} & \multirow{2}{*}{0.363} & \multirow{2}{*}{0.327} & \multirow{2}{*}{12.2} \\
\hline & QS6 & 369992.40 & 0.30 & & & & & & & & \\
\hline \multirow{4}{*}{4} & QS7 & 520750.20 & 0.42 & \multirow{2}{*}{0.41} & \multirow{2}{*}{0.61} & \multirow{2}{*}{0.053} & \multirow{2}{*}{5.000} & \multirow{2}{*}{2.675} & \multirow{2}{*}{0.471} & \multirow{2}{*}{0.424} & \multirow{2}{*}{15.8} \\
\hline & QS8 & 502271.16 & 0.40 & & & & & & & & \\
\hline & & & & & & & & & $\mathrm{AVG}=$ & 0.379 & 14.2 \\
\hline & & & & & & & & & & StdDev $=$ & 2.2 \\
\hline
\end{tabular}

Sample Calculation:

- $\quad \%$ SRS (Sugar Recovery Standards) = (conc. detected by HPLC, g/L) x 100/( known conc. of sugar before hydrolysis, g/L) = $6.74 \mathrm{~g} / \mathrm{L}$ x 100/ (0.1/0.01) g/L = 67.4\%; $87 \mathrm{ml}=0.087 \mathrm{~L}-3 \mathrm{ml}$ acid $+84 \mathrm{ml} \mathrm{DDW}$

- $\quad$ Corrected Glucose $=0.44 \mathrm{~g} / \mathrm{L} / 0.674(\mathrm{SRS})=0.653 \mathrm{~g} / \mathrm{L}$

- $\quad$ Glucose in 0.3 g QS Sample $=0.65$ g/L x $0.087 \mathrm{~L}=0.0565 \mathrm{~g}$

- $\quad$ Pretreated SSO per bottle $=5 \mathrm{~g}$

- $\quad$ Pretreated SSO per Bottle without Moisture = $5 \mathrm{~g}$ x 0.535 (\%TS from Moisture Contents table) = $2.675 \mathrm{~g}$

- $\quad$ Glucose per Pretreated Dry SSO Bottle $=(2.675$ g x 0.0565 g) / 0.3 g $=0.504$ g

- $\quad$ Glucan per Pretreated Dry SSO Bottle $=0.504 \mathrm{~g}$ x $0.9=0.454 \mathrm{~g}$

- $\quad$ Glucan Yield per 5 g SSO Bottle $=0.454$ g x $100 / 2.675$ g $=16.96 \%$ 
Cellulase Activitiy of Accelerase 1500

Date: April 15, 2013

Table 4: Glucose standards

\begin{tabular}{cccccc}
\hline $\begin{array}{c}\text { Standards } \\
\#\end{array}$ & $\begin{array}{c}\text { Glucose } \\
\text { stock } \\
(\mathrm{ml})\end{array}$ & $\begin{array}{c}\text { Citrate } \\
\text { Buffer } \\
(\mathrm{ml})\end{array}$ & Dilution & $\begin{array}{c}\text { Glucose } \\
(\mathrm{mg} / 0,5\end{array}$ & $\begin{array}{c}\text { Abs } \\
\text { @540nm }\end{array}$ \\
\hline S1 & 1 & 0.5 & $1 / 15$ & 3.35 & 0.605 \\
S2 & 1 & 1 & $1 / 2$ & 2.5 & 0.473 \\
S3 & 1 & 2 & $1 / 3$ & 1.65 & 0.317 \\
S4 & 1 & 4 & $1 / 4$ & 1 & 0.179 \\
\hline
\end{tabular}

Table 5: Glucose responses vs different concentrations

\begin{tabular}{cccc}
\hline Dilution \# & $\begin{array}{c}\text { Citrate buffer } \\
(\mathrm{ml})\end{array}$ & $\begin{array}{c}1: 20 \text { Enzyme } \\
(\mathrm{ml})\end{array}$ & Conc. \\
\hline 1 & 33 & 7 & 0.00875 \\
2 & 34 & 6 & 0.0075 \\
3 & 36 & 4 & 0.005 \\
4 & 37 & 3 & 0.00375 \\
5 & 38 & 2 & 0.0025 \\
\hline
\end{tabular}




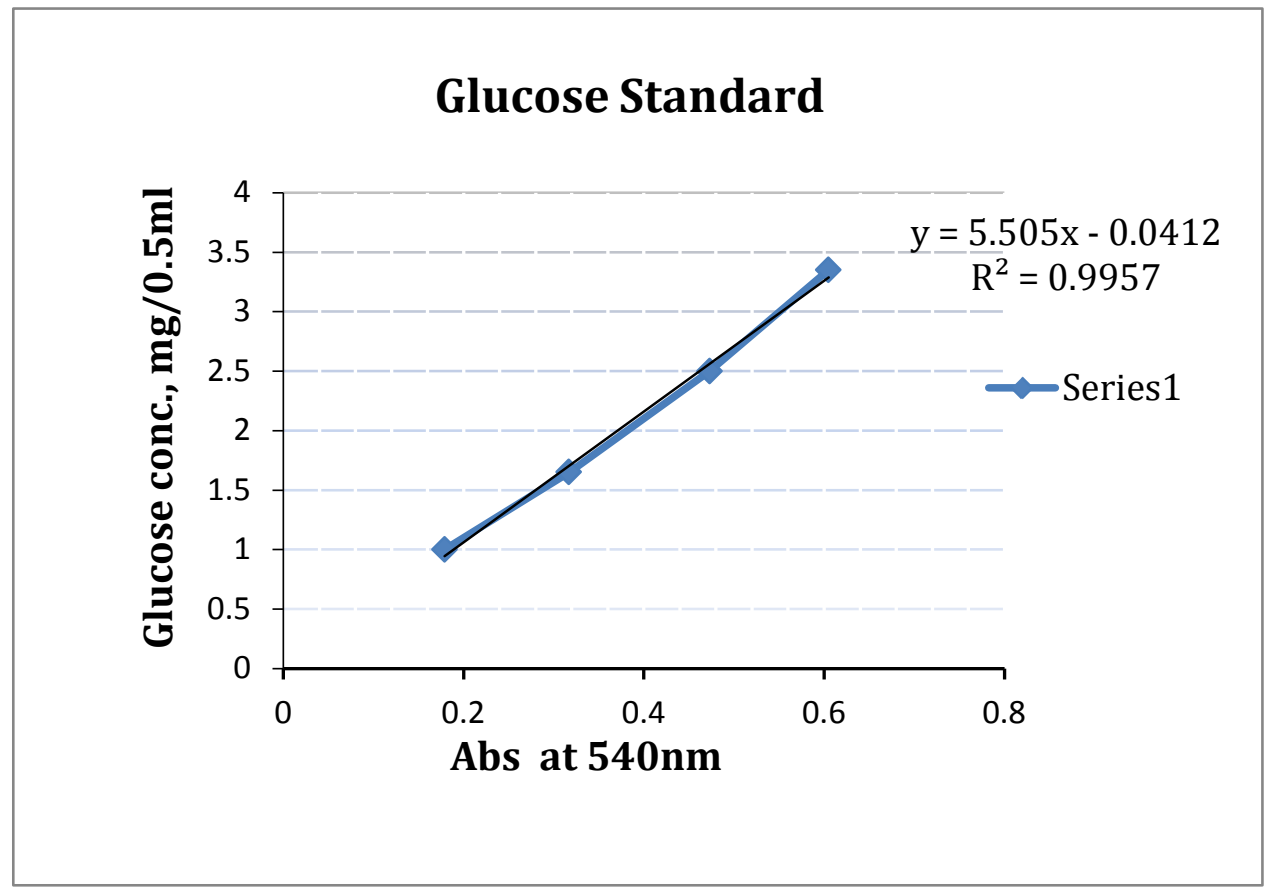

Figure 2: Glucose standards plot

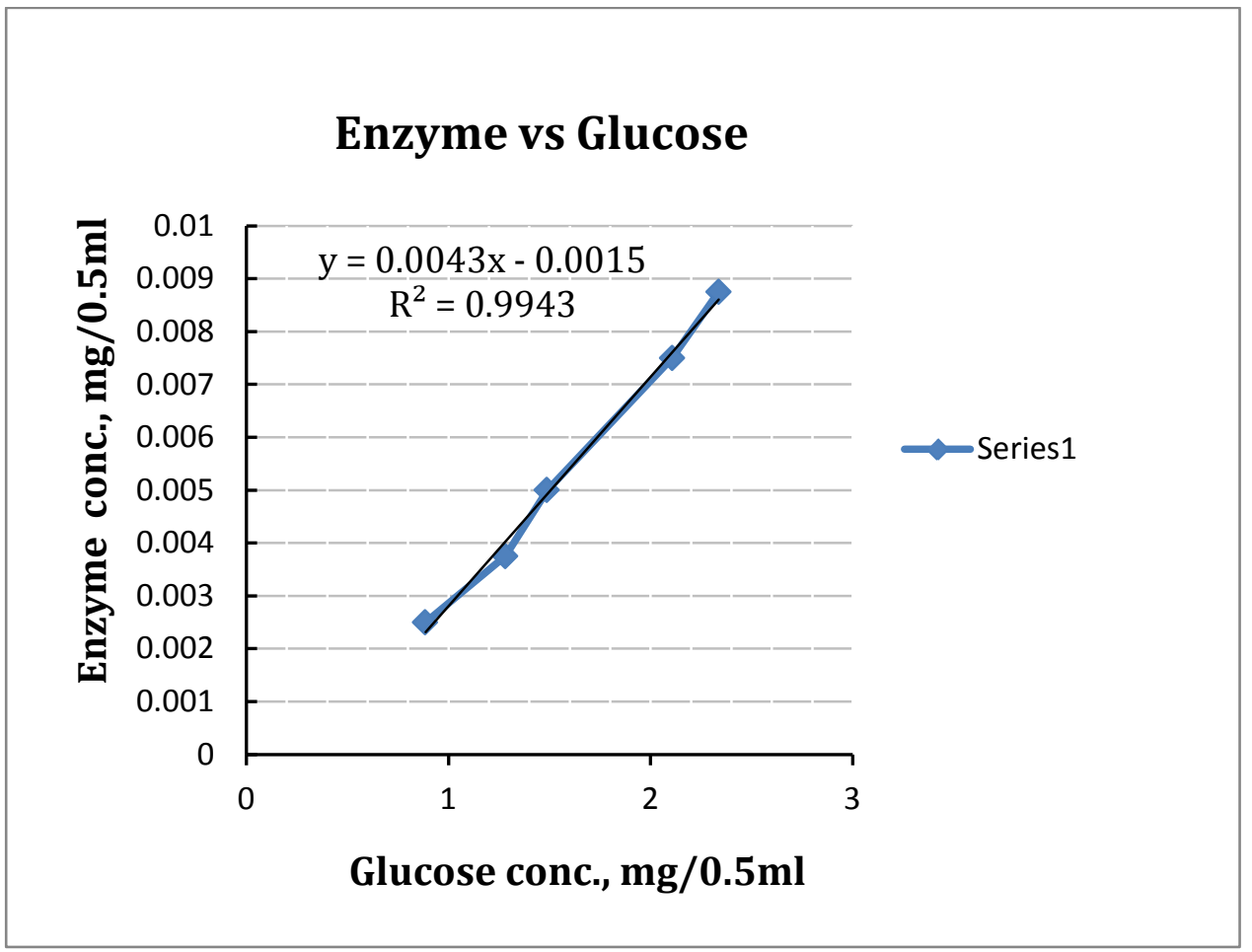

Figure 3: Enzyme vs Glucose plot 
Table 6: Glucose readings vs dilutions factor

\begin{tabular}{|c|c|c|c|c|c|c|c|}
\hline $\begin{array}{c}\text { Dilution } \\
\qquad \#\end{array}$ & $\begin{array}{c}\text { Sample } \\
\#\end{array}$ & Abs@540nm & $\begin{array}{c}\text { Average } \\
\text { Abs }\end{array}$ & $\begin{array}{c}\text { Enzyme } \\
\text { blank }\end{array}$ & $\begin{array}{c}\text { Substrate } \\
\text { blank }\end{array}$ & $\begin{array}{c}\text { Corrected } \\
\text { Abs } \\
\text { @540nm }\end{array}$ & $\begin{array}{c}\text { Glucose } \\
(\mathrm{mg} / 0.5 \mathrm{ml})\end{array}$ \\
\hline \multirow{4}{*}{1} & 1.1 & 0.419 & \multirow{4}{*}{0.433} & \multirow{4}{*}{0.001} & \multirow{4}{*}{0} & \multirow{4}{*}{0.432} & \multirow{4}{*}{2.337} \\
\hline & 1.2 & 0.42 & & & & & \\
\hline & 1.3 & 0.46 & & & & & \\
\hline & 2.1 & 0.374 & & & & & \\
\hline \multirow[t]{3}{*}{2} & 2.2 & 0.396 & \multirow[t]{3}{*}{0.391} & \multirow[t]{3}{*}{0.001} & & \multirow[t]{3}{*}{0.390} & \multirow[t]{3}{*}{2.108} \\
\hline & 2.3 & 0.404 & & & & & \\
\hline & 3.1 & 0.266 & & & & & \\
\hline \multirow[t]{3}{*}{3} & 3.2 & 0.277 & \multirow[t]{3}{*}{0.278} & \multirow[t]{3}{*}{0} & & \multirow[t]{3}{*}{0.278} & \multirow[t]{3}{*}{1.488} \\
\hline & 3.3 & 0.29 & & & & & \\
\hline & 4.1 & 0.259 & & & & & \\
\hline \multirow[t]{3}{*}{4} & 4.2 & 0.219 & \multirow[t]{3}{*}{0.240} & \multirow[t]{3}{*}{0} & & \multirow[t]{3}{*}{0.240} & \multirow[t]{3}{*}{1.282} \\
\hline & 4.3 & 0.243 & & & & & \\
\hline & 5.1 & 0.176 & & & & & \\
\hline \multirow[t]{2}{*}{5} & 5.2 & 0.154 & \multirow[t]{2}{*}{0.168} & \multirow[t]{2}{*}{0} & & \multirow[t]{2}{*}{0.168} & \multirow[t]{2}{*}{0.886} \\
\hline & 5.3 & 0.175 & & & & & \\
\hline
\end{tabular}


Table 7: Enzyme concentration vs glucose

\begin{tabular}{ccc}
\hline Dilution \# & Enzyme conc & Glucose (mg/0.5 ml) \\
& & \\
\hline 1 & 0.00875 & 2.337 \\
3 & 0.0075 & 2.108 \\
4 & 0.005 & 1.488 \\
5 & 0.00375 & 1.282 \\
\hline
\end{tabular}

Calculation of FPU from graph at 2.0 glucose using equation $\mathrm{y}=(0.004 \mathrm{x} 2-0.001)$

\begin{tabular}{ll}
\hline $0.0075-\mathrm{X}$ & $2.108-2.0$ \\
$0.0075-0.005$ & $2.108-1.488$ \\
\hline
\end{tabular}

$\mathrm{X}=0.00706 \rightarrow \mathrm{FPU}=52.4$ 
Enzymatic hydrolysis on SSO

Glucose Yield from Enzymatic Hydrolysis - HPLC Results

Date: June 07, 2013

Table 8: Glucose standards

\begin{tabular}{ccc}
\hline Standards & Concentration (g/L) & $\begin{array}{c}\text { Glucose } \\
\text { Area (uV.S) }\end{array}$ \\
\hline S1 & 20 & 25526794.40 \\
S2 & 5 & 5483267.48 \\
S3 & 2 & 2208890.80 \\
S4 & 1 & 1302174.20 \\
\hline
\end{tabular}

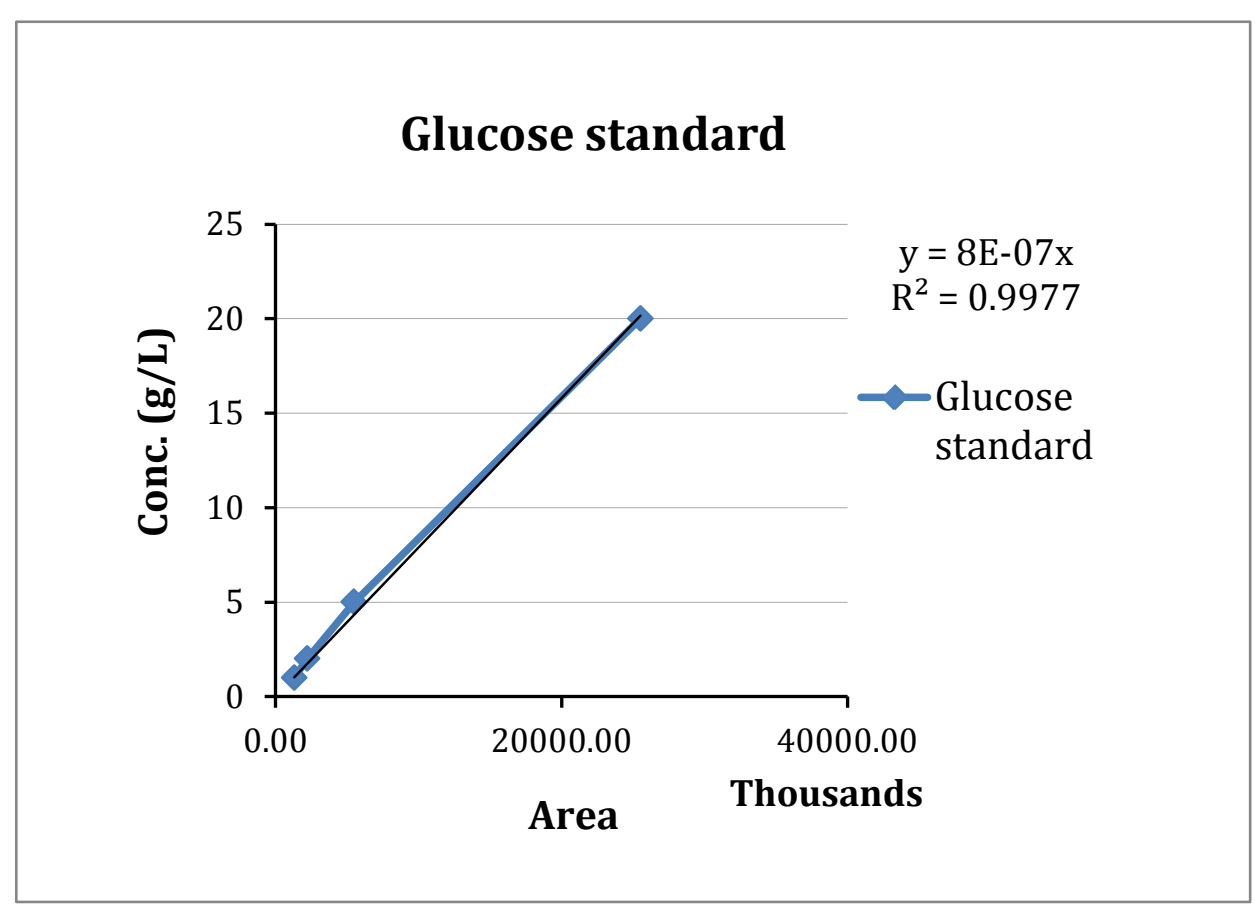

Figure 4: Glucose standards plot 
Table 9: HPLC data from enzymatic hydrolysis test

\begin{tabular}{|c|c|c|c|c|c|c|c|}
\hline \multirow[t]{2}{*}{$\begin{array}{c}\text { Bottle } \\
\text { Number }\end{array}$} & \multirow[t]{2}{*}{$\begin{array}{l}\text { Sample } \\
\text { Name }\end{array}$} & \multirow[t]{2}{*}{$\begin{array}{l}\text { Time } \\
\text { (h) }\end{array}$} & $\begin{array}{l}\text { Glucose } \\
\text { (HPLC) }\end{array}$ & $\begin{array}{l}\text { Glucose } \\
\text { (HPLC) }\end{array}$ & \multirow{2}{*}{$\begin{array}{c}\text { Average* } \\
\pm \text { Std. Dev. } \\
\text { (g/L) }\end{array}$} & \multirow{2}{*}{$\begin{array}{c}\text { Glucose } \\
\text { Yield** } \\
(\%)\end{array}$} & \multirow{2}{*}{$\begin{array}{c}\text { Corrected } \\
* * * \\
\text { Glucose } \\
\text { Yield } \\
(\%)\end{array}$} \\
\hline & & & Area( $\mu$ V.S $)$ & $\begin{array}{l}\text { Conc. } \\
\text { (g/L) }\end{array}$ & & & \\
\hline 1 & EH0-1 & 0 & 10392161.96 & 9.35 & 9.28 & 42.18 & 67.38 \\
\hline 2 & EH0-2 & & 9593459.50 & 8.63 & \pm 0.39 & & \\
\hline 3 & EH0-3 & & 10484796.95 & 9.44 & & & \\
\hline 4 & EH0-4 & & 10375601.71 & 9.34 & & & \\
\hline 5 & EH0-5 & & 10141384.28 & 9.13 & & & \\
\hline 6 & EH0-6 & & 10872933.64 & 9.79 & & & \\
\hline 1 & EH12-1 & 12 & 9189055.08 & 8.27 & 8.33 & 37.86 & 60.49 \\
\hline 2 & EH12-2 & & 9579356.30 & 8.62 & \pm 0.69 & & \\
\hline 3 & EH12-3 & & 8979810.23 & 8.08 & & & \\
\hline 4 & EH12-4 & & 8953359.26 & 8.06 & & & \\
\hline 5 & EH12-5 & & 8866237.90 & 7.98 & & & \\
\hline 6 & EH12-6 & & 9966604.55 & 8.97 & & & \\
\hline 1 & EH24-1 & 24 & 7765087.75 & 6.99 & 6.82 & 30.98 & 49.50 \\
\hline 2 & EH24-2 & & 8111969.32 & 7.30 & \pm 0.82 & & \\
\hline 3 & EH24-3 & & 6810284.52 & 6.13 & & & \\
\hline 4 & EH24-4 & & 7086792.14 & 6.38 & & & \\
\hline 5 & EH24-5 & & 7469906.85 & 6.72 & & & \\
\hline 6 & EH24-6 & & 8197735.36 & 7.38 & & & \\
\hline 1 & EH48-1 & 48 & 1357805.17 & 1.22 & 1.57 & 7.15 & 11.43 \\
\hline 2 & EH48-2 & & 1763737.14 & 1.59 & \pm 0.79 & & \\
\hline 3 & EH48-3 & & 1977832.06 & 1.78 & & & \\
\hline 4 & EH48-4 & & 326430.38 & 0.29 & & & \\
\hline 5 & EH48-5 & & 1519931.15 & 1.37 & & & \\
\hline 6 & EH48-6 & & 3544895.30 & 3.19 & & & \\
\hline
\end{tabular}




\section{Fermentation on SSO}

Date: May 07, 2013

Sugar content for each 5g sample: 20 g/L Glucan (or 22 g/L Glucose)

Table 10: Ethanol standard data

\begin{tabular}{|c|c|c|c|}
\hline Standards & $\begin{array}{l}\text { Concentration } \\
\text { (mM) }\end{array}$ & $\begin{array}{c}\text { Glucose } \\
\text { Area (uV.S) }\end{array}$ & \\
\hline S1 & 1 & $\mathrm{n} / \mathrm{a}$ & $\mathrm{n} / \mathrm{a}$ \\
\hline S1-1 & & $\mathrm{n} / \mathrm{a}$ & \\
\hline S2 & 2 & 120340.88 & 119125.88 \\
\hline S2-1 & & 117910.88 & \\
\hline S3 & 5 & 330331.42 & 301351.15 \\
\hline S3-1 & & 272370.87 & \\
\hline S4 & 10 & 600482.72 & 578400.96 \\
\hline S4- & & 556319.20 & \\
\hline S5 & 20 & 1280233.40 & 1172104.10 \\
\hline S5-1 & & 1063974.80 & \\
\hline S6 & 50 & 3276946.95 & 3126817.87 \\
\hline S6-1 & & 2976688.78 & \\
\hline
\end{tabular}




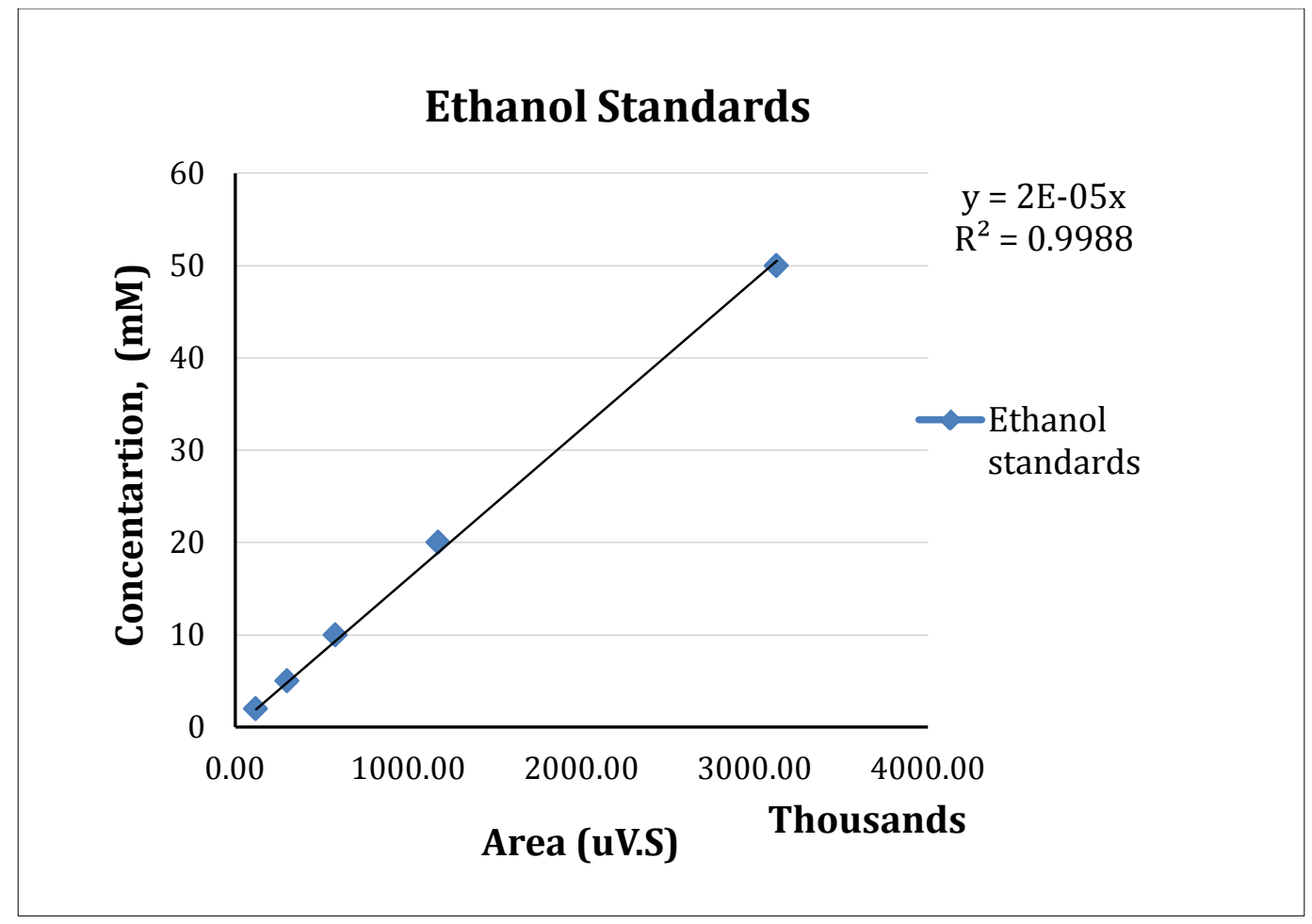

Figure 5: Ethanol standards plot 
Date: May 07, 2013

Table 11: Ethanol yield data from fermentation test

\begin{tabular}{|c|c|c|c|c|c|c|c|c|}
\hline \multirow{3}{*}{$\begin{array}{c}\text { Bottle } \\
\text { Number }\end{array}$} & \multirow{3}{*}{$\begin{array}{c}\text { Sample } \\
\text { Name }\end{array}$} & \multirow{3}{*}{$\begin{array}{l}\text { Time } \\
\text { (hr) }\end{array}$} & Ethanol & Ethanol & Ethanol & \multirow{3}{*}{$\begin{array}{c}\text { Average* } \\
\text { Ethanol Conc. } \\
\pm \text { Std. Dev. } \\
\text { (g/L) }\end{array}$} & \multirow{3}{*}{$\begin{array}{l}\text { Corrected** } \\
\text { Average } \\
\text { Ethanol } \\
\text { Conc. } \\
(\mathrm{g} / \mathrm{L})\end{array}$} & \multirow{3}{*}{$\begin{array}{c}\text { Ethanol } \\
\text { Yield*** } \\
(\%)\end{array}$} \\
\hline & & & (HPLC) & (HPLC) & (HPLC) & & & \\
\hline & & & Area( $\mu$ V.S $)$ & $\begin{array}{l}\text { Con. } \\
(\mathrm{mM})\end{array}$ & $\begin{array}{l}\text { Con. } \\
(\mathrm{g} / \mathrm{L})\end{array}$ & & & \\
\hline 1 & FE0-1 & 0 & 37016888.42 & 740.34 & 34.11 & 24.27 & 38.77 & 96.5 \\
\hline 3 & FE0-2 & & 29498528.60 & 589.97 & 27.18 & \pm 3.80 & & \\
\hline 5 & FE0-3 & & 12496254.80 & 249.93 & 11.51 & & & \\
\hline 1 & FE12-1 & 12 & 29687795.95 & 593.76 & 27.35 & 23.15 & 36.99 & 92.1 \\
\hline 3 & FE12-2 & & 31453122.00 & 629.06 & 28.98 & \pm 5.97 & & \\
\hline 5 & FE12-3 & & 14246734.09 & 284.93 & 13.13 & & & \\
\hline 1 & FE24-1 & 24 & 32356585.63 & 647.13 & 29.81 & 27.83 & 44.46 & 110.6 \\
\hline 2 & FE24-2 & & 31799065.63 & 635.98 & 29.30 & \pm 5.51 & & \\
\hline 3 & FE24-3 & & 26453535.85 & 529.07 & 24.37 & & & \\
\hline 1 & FE48-1 & 48 & 31357550.84 & 627.15 & 28.89 & 33.32 & 53.23 & 132.5 \\
\hline 2 & FE48-2 & & 31582077.30 & 631.64 & 29.10 & \pm 6.87 & & \\
\hline 3 & FE48-3 & & 45546744.41 & 910.93 & 41.97 & & & \\
\hline
\end{tabular}

*** Use \% theoretical ethanol yield equation: where $\mathrm{f}=(3.64 \mathrm{~g} / 3.64 \mathrm{~g})$ and biomass $=(3.64 \mathrm{~g} / 0.082 \mathrm{~L})$

$1000 \mathrm{~g}$ or $1 \mathrm{Kg}$ biomass $=[(33.32 \mathrm{~g} / \mathrm{L} \times 0.082 \mathrm{~L}) \times 1000 \mathrm{~g}] / 5 \mathrm{~g}=546.448 \mathrm{~g}$ Ethanol 
Date: June 06, 2013

Table 12: Ethanol yield data from fermentation test

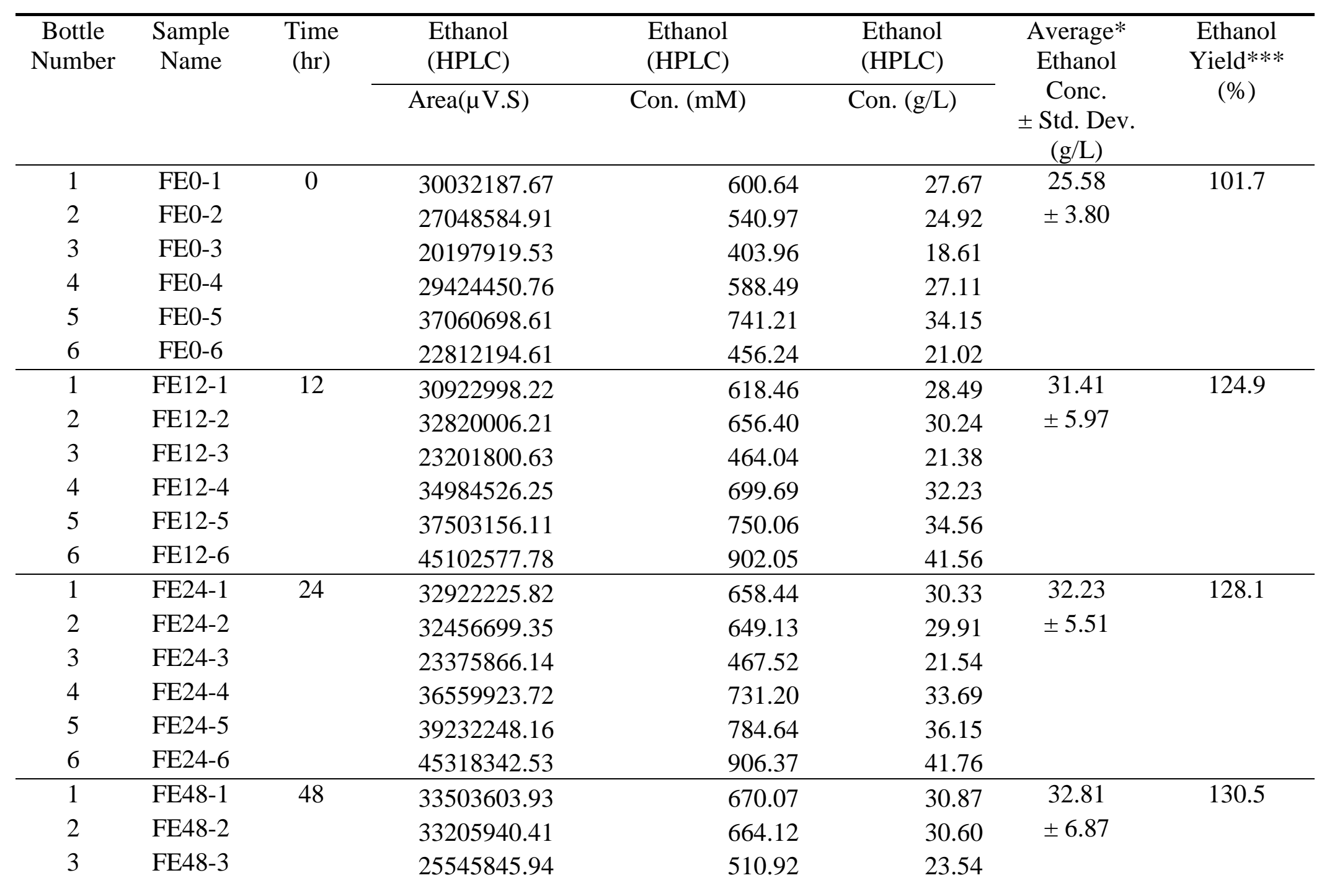




\begin{tabular}{|c|c|c|c|c|c|c|c|}
\hline \multirow[t]{2}{*}{$\begin{array}{c}\text { Bottle } \\
\text { Number }\end{array}$} & \multirow[t]{2}{*}{$\begin{array}{c}\text { Sample } \\
\text { Name }\end{array}$} & \multirow[t]{5}{*}{$\begin{array}{l}\text { Time } \\
\text { (hr) }\end{array}$} & $\begin{array}{l}\text { Ethanol } \\
\text { (HPLC) }\end{array}$ & $\begin{array}{l}\text { Ethanol } \\
\text { (HPLC) }\end{array}$ & $\begin{array}{l}\text { Ethanol } \\
\text { (HPLC) }\end{array}$ & \multirow{2}{*}{$\begin{array}{c}\text { Average* } \\
\text { Ethanol } \\
\text { Conc. } \\
\pm \text { Std. Dev. } \\
\text { (g/L) }\end{array}$} & \multirow{5}{*}{$\begin{array}{c}\text { Ethanol } \\
\text { Yield*** } \\
\text { (\%) }\end{array}$} \\
\hline & & & Area( $\mu$ V.S $)$ & Con. (mM) & Con. (g/L) & & \\
\hline 4 & FE48-4 & & 36075988.81 & 721.52 & 33.24 & & \\
\hline 5 & FE48-5 & & 39949968.81 & 799.00 & 36.81 & & \\
\hline 6 & FE48-6 & & 45380957.21 & 907.62 & 41.81 & & \\
\hline
\end{tabular}


Date: June 13, 2013

Table 13: Ethanol yield data from fermentation test

\begin{tabular}{|c|c|c|c|c|c|c|c|}
\hline \multirow[t]{2}{*}{$\begin{array}{c}\text { Bottle } \\
\text { Number }\end{array}$} & \multirow[t]{2}{*}{$\begin{array}{l}\text { Sample } \\
\text { Name }\end{array}$} & \multirow[t]{2}{*}{$\begin{array}{l}\text { Time } \\
\text { (h) }\end{array}$} & $\begin{array}{l}\text { Ethanol } \\
\text { (HPLC) }\end{array}$ & $\begin{array}{l}\text { Ethanol } \\
\text { (HPLC) }\end{array}$ & $\begin{array}{l}\text { Ethanol } \\
\text { (HPLC) }\end{array}$ & \multirow{2}{*}{$\begin{array}{c}\text { Average* } \\
\text { Ethanol } \\
\text { Conc. } \\
\pm \text { Std. Dev. } \\
\text { (g/L) }\end{array}$} & \multirow{2}{*}{$\begin{array}{c}\text { Ethanol } \\
\text { Yield*** } \\
(\%)\end{array}$} \\
\hline & & & $\operatorname{Area}(\mu \mathrm{V} . \mathrm{S})$ & Con. (mM) & Con. (g/L) & & \\
\hline 1 & FE0-1 & 0 & 25008355.52 & 500.17 & 23.04 & 20.20 & 80.3 \\
\hline 2 & FE0-2 & & 22366405.21 & 447.33 & 20.61 & \pm 3.80 & \\
\hline 3 & FE0-3 & & 22679645.00 & 453.59 & 20.90 & & \\
\hline 4 & FE0-4 & & 19456425.36 & 389.13 & 17.93 & & \\
\hline 5 & FE0-5 & & 17194847.38 & 343.90 & 15.84 & & \\
\hline 6 & FE0-6 & & 26464365.01 & 529.29 & 24.38 & & \\
\hline 7 & FE0-7 & & 28536034.18 & 570.72 & 26.29 & & \\
\hline 8 & FE0-8 & & 21376668.65 & 427.53 & 19.70 & & \\
\hline 9 & FE0-9 & & 10316929.62 & 206.34 & 9.51 & & \\
\hline 10 & FE0-10 & & 25798750.81 & 515.98 & 23.77 & & \\
\hline 1 & FE12-1 & 12 & 29222315.21 & 584.45 & 26.93 & 27.10 & 107.7 \\
\hline 2 & FE12-2 & & 28481435.81 & 569.63 & 26.24 & \pm 5.97 & \\
\hline 3 & FE12-3 & & 28734987.61 & 574.70 & 26.48 & & \\
\hline 4 & FE12-4 & & 26886343.45 & 537.73 & 24.77 & & \\
\hline 5 & FE12-5 & & 27505012.56 & 550.10 & 25.34 & & \\
\hline 6 & FE12-6 & & 29881089.72 & 597.62 & 27.53 & & \\
\hline 7 & FE12-7 & & 30084484.41 & 601.69 & 27.72 & & \\
\hline 8 & FE12-8 & & 35646373.16 & 712.93 & 32.84 & & \\
\hline 9 & FE12-9 & & 26137263.62 & 522.75 & 24.08 & & \\
\hline 10 & FE12-10 & & 31493054.91 & 629.86 & 29.02 & & \\
\hline 1 & FE24-1 & 24 & 33397917.61 & 667.96 & 30.77 & 29.27 & 116.4 \\
\hline
\end{tabular}




\begin{tabular}{|c|c|c|c|c|c|c|c|}
\hline \multirow[t]{2}{*}{$\begin{array}{c}\text { Bottle } \\
\text { Number }\end{array}$} & \multirow[t]{2}{*}{$\begin{array}{l}\text { Sample } \\
\text { Name }\end{array}$} & \multirow[t]{11}{*}{$\begin{array}{l}\text { Time } \\
\text { (h) }\end{array}$} & $\begin{array}{l}\text { Ethanol } \\
\text { (HPLC) }\end{array}$ & $\begin{array}{l}\text { Ethanol } \\
\text { (HPLC) }\end{array}$ & $\begin{array}{l}\text { Ethanol } \\
\text { (HPLC) }\end{array}$ & \multirow{2}{*}{$\begin{array}{c}\text { Average* } \\
\text { Ethanol } \\
\text { Conc. } \\
\pm \text { Std. Dev. } \\
\text { (g/L) }\end{array}$} & \multirow{11}{*}{$\begin{array}{c}\text { Ethanol } \\
\text { Yield*** } \\
(\%)\end{array}$} \\
\hline & & & Area( $\mu$ V.S $)$ & Con. (mM) & Con. (g/L) & & \\
\hline 2 & FE24-2 & & 30184427.61 & 603.69 & 27.81 & \pm 5.51 & \\
\hline 3 & FE24-3 & & 30290380.84 & 605.81 & 27.91 & & \\
\hline 4 & FE24-4 & & 32953400.41 & 659.07 & 30.36 & & \\
\hline 5 & FE24-5 & & 33919696.24 & 678.39 & 31.25 & & \\
\hline 6 & FE24-6 & & 31896572.89 & 637.93 & 29.39 & & \\
\hline 7 & FE24-7 & & 30901551.58 & 618.03 & 28.47 & & \\
\hline 8 & FE24-8 & & 35279318.05 & 705.59 & 32.51 & & \\
\hline 9 & FE24-9 & & 27556285.79 & 551.13 & 25.39 & & \\
\hline 10 & FE24-10 & & 31339491.77 & 626.79 & 28.88 & & \\
\hline 1 & FE48-1 & 48 & 35013714.81 & 700.27 & 32.26 & 30.01 & 119.3 \\
\hline 2 & FE48-2 & & 30918388.42 & 618.37 & 28.49 & \pm 6.87 & \\
\hline 3 & FE48-3 & & 30449180.17 & 608.98 & 28.06 & & \\
\hline 4 & FE48-4 & & 33190145.61 & 663.80 & 30.58 & & \\
\hline 5 & FE48-5 & & 34721738.49 & 694.43 & 31.99 & & \\
\hline 6 & FE48-6 & & 31344345.17 & 626.89 & 28.88 & & \\
\hline 7 & FE48-7 & & 31024674.79 & 620.49 & 28.59 & & \\
\hline 8 & FE48-8 & & 36780882.21 & 735.62 & 33.89 & & \\
\hline 9 & FE48-9 & & 30560026.81 & 611.20 & 28.16 & & \\
\hline 10 & FE48-10 & & 31658412.46 & 633.17 & 29.17 & & \\
\hline
\end{tabular}




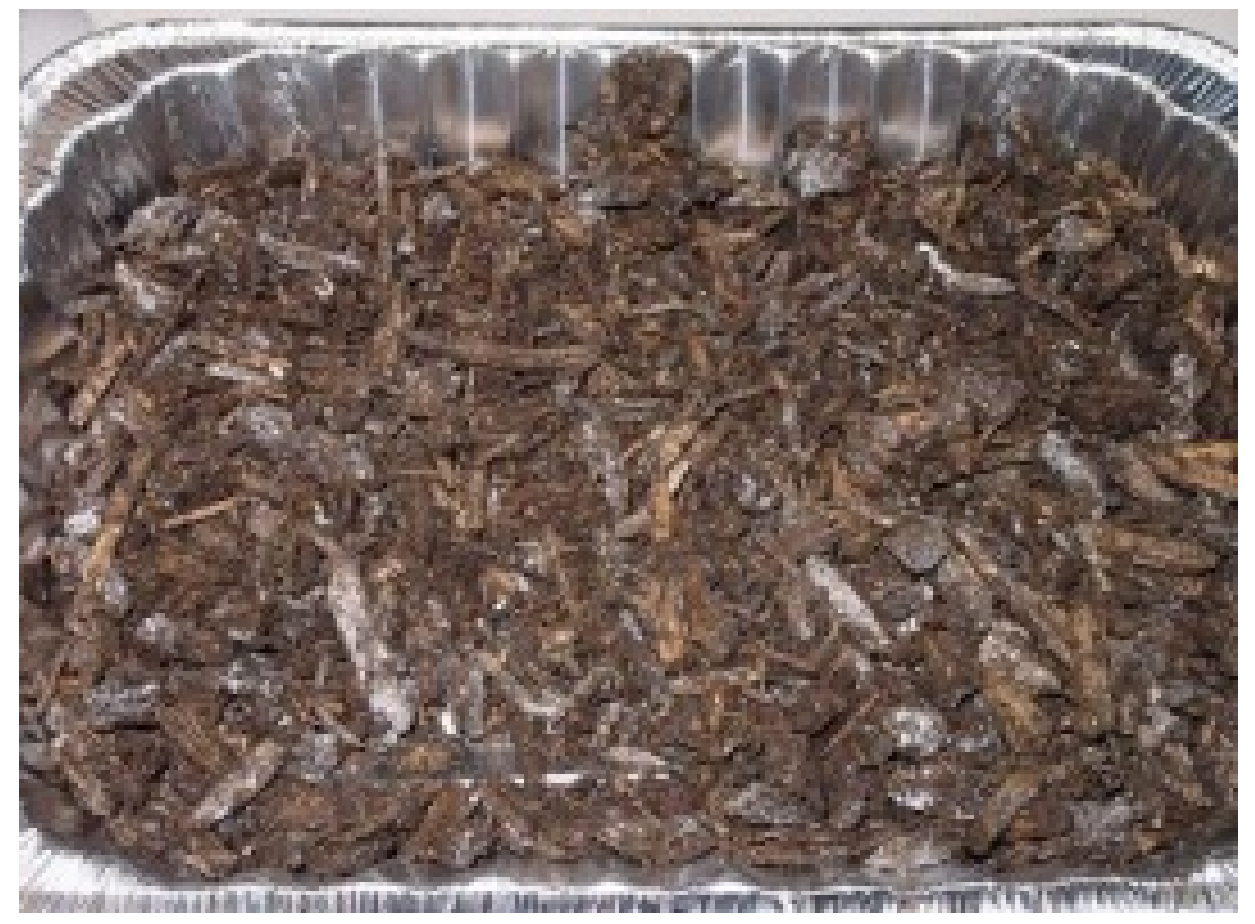

Picture 1: Dried SSO sample

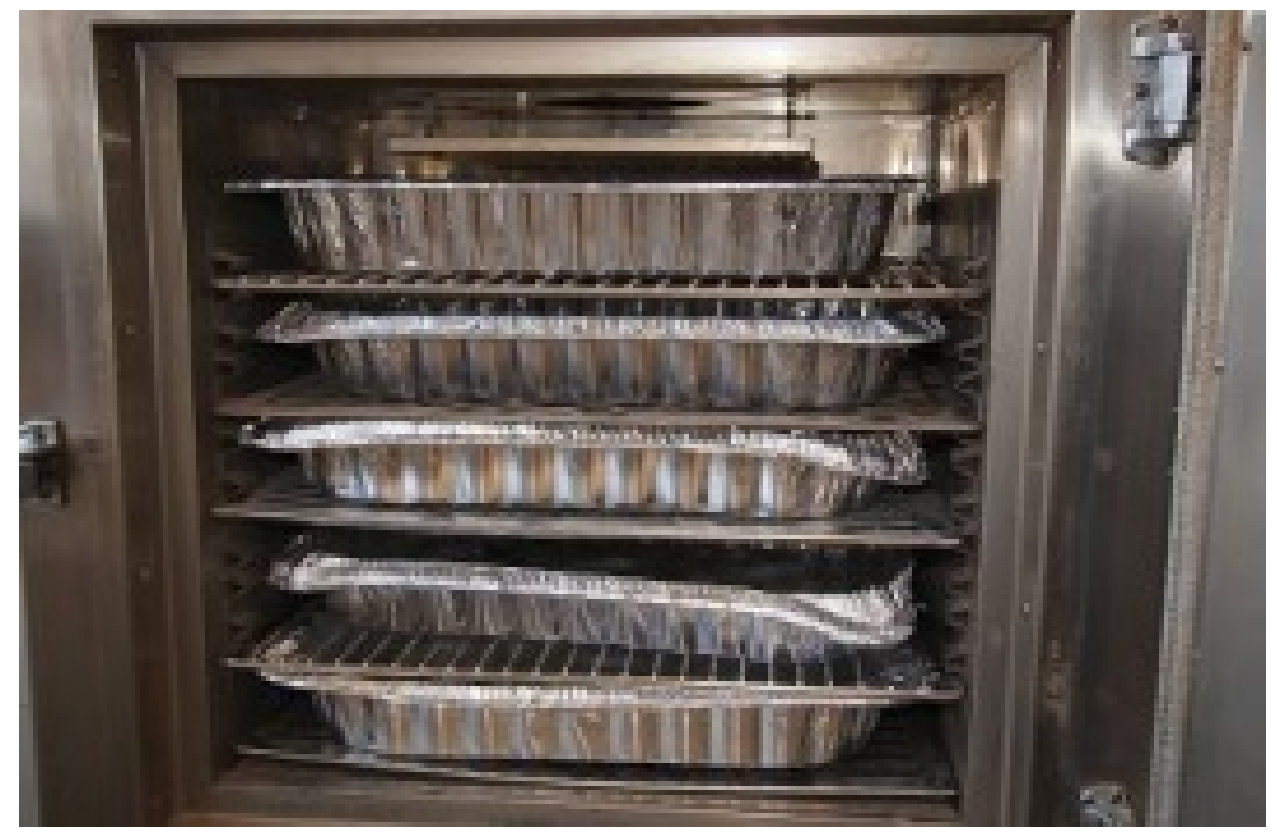

Picture 2: SSO samples in the stove-oven 


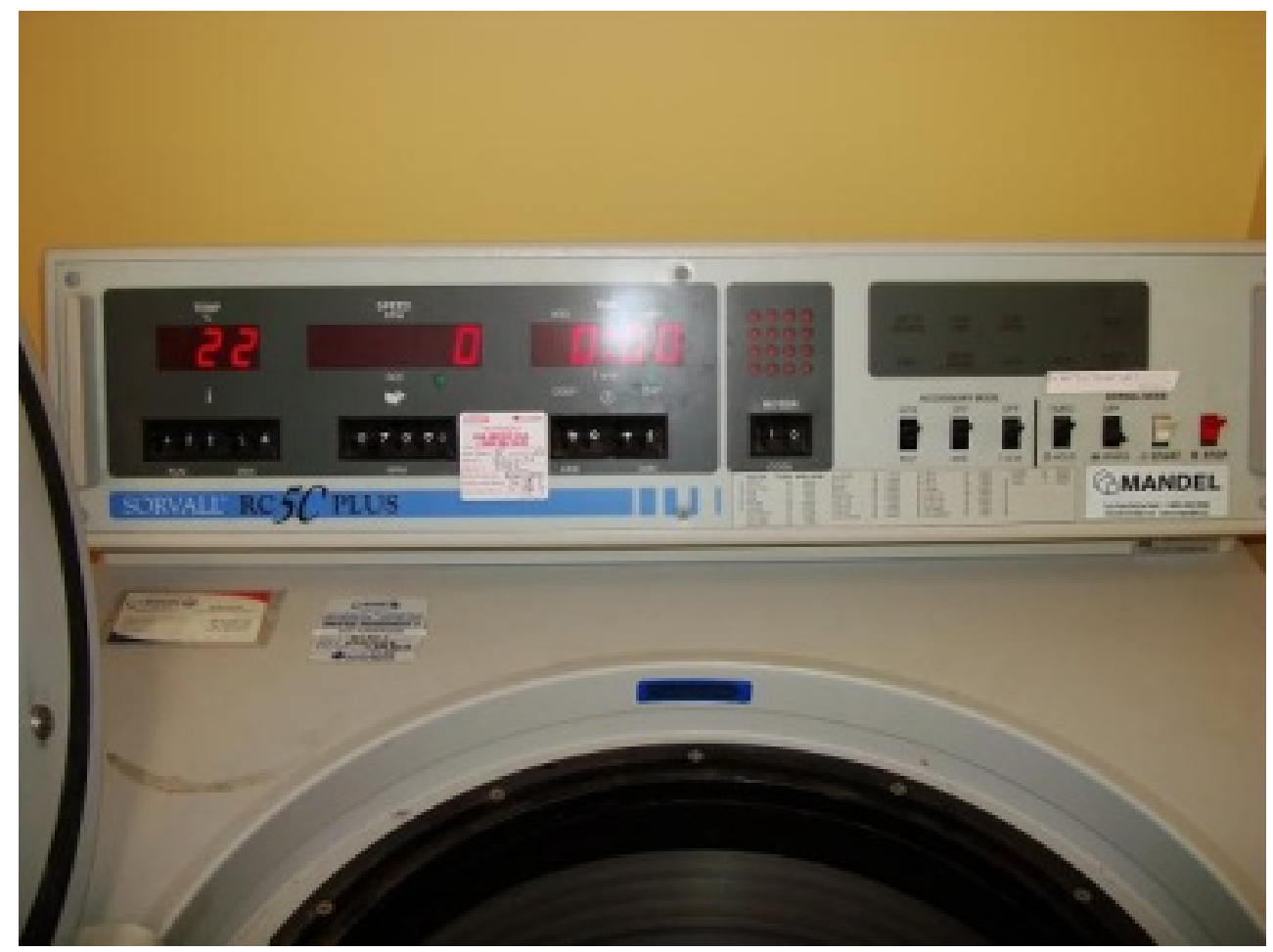

Picture 3: Centrifuge Sorvall RC 5C

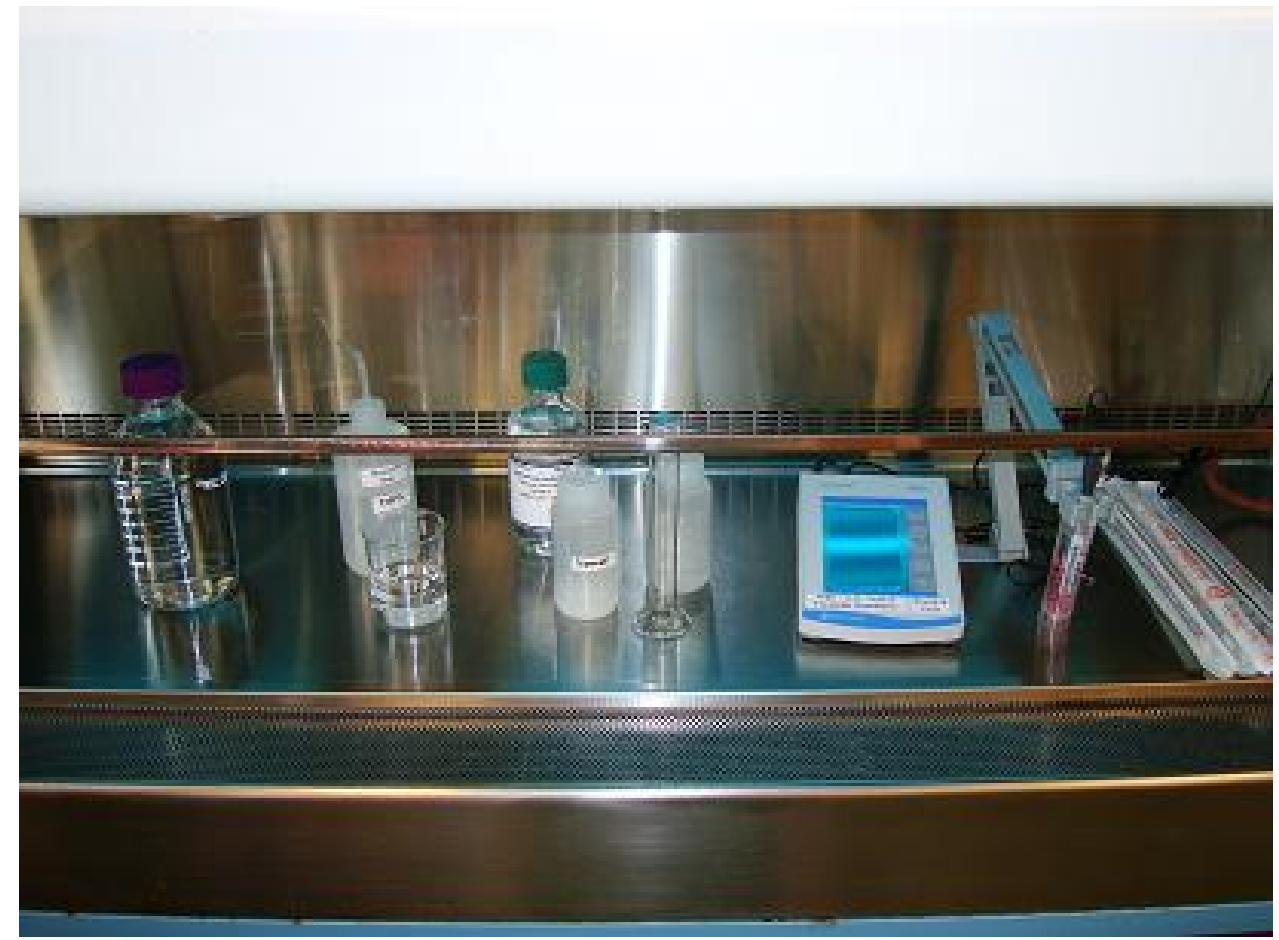

Picture 4: Biohood 


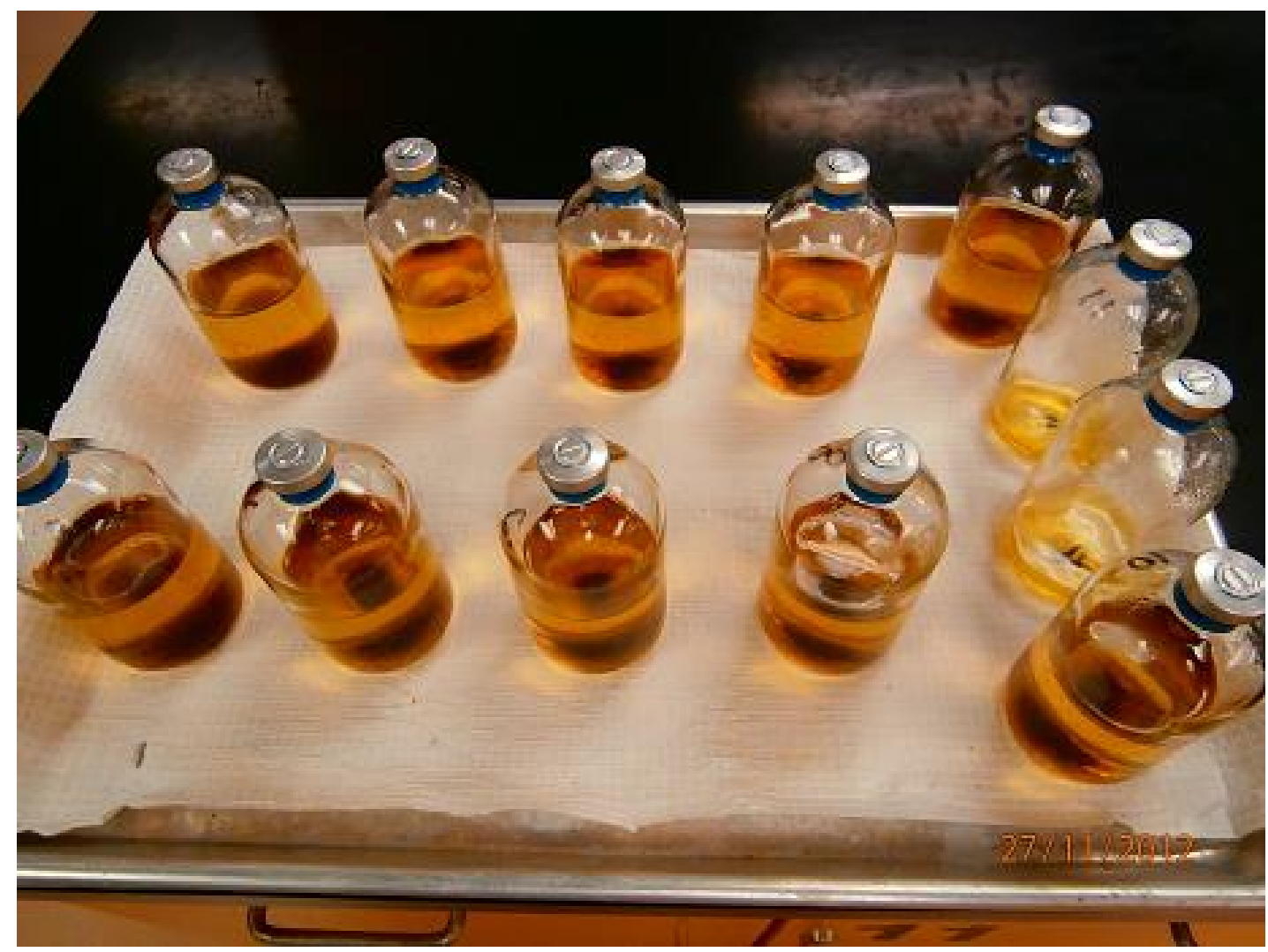

Picture 5: Cellulase activity test

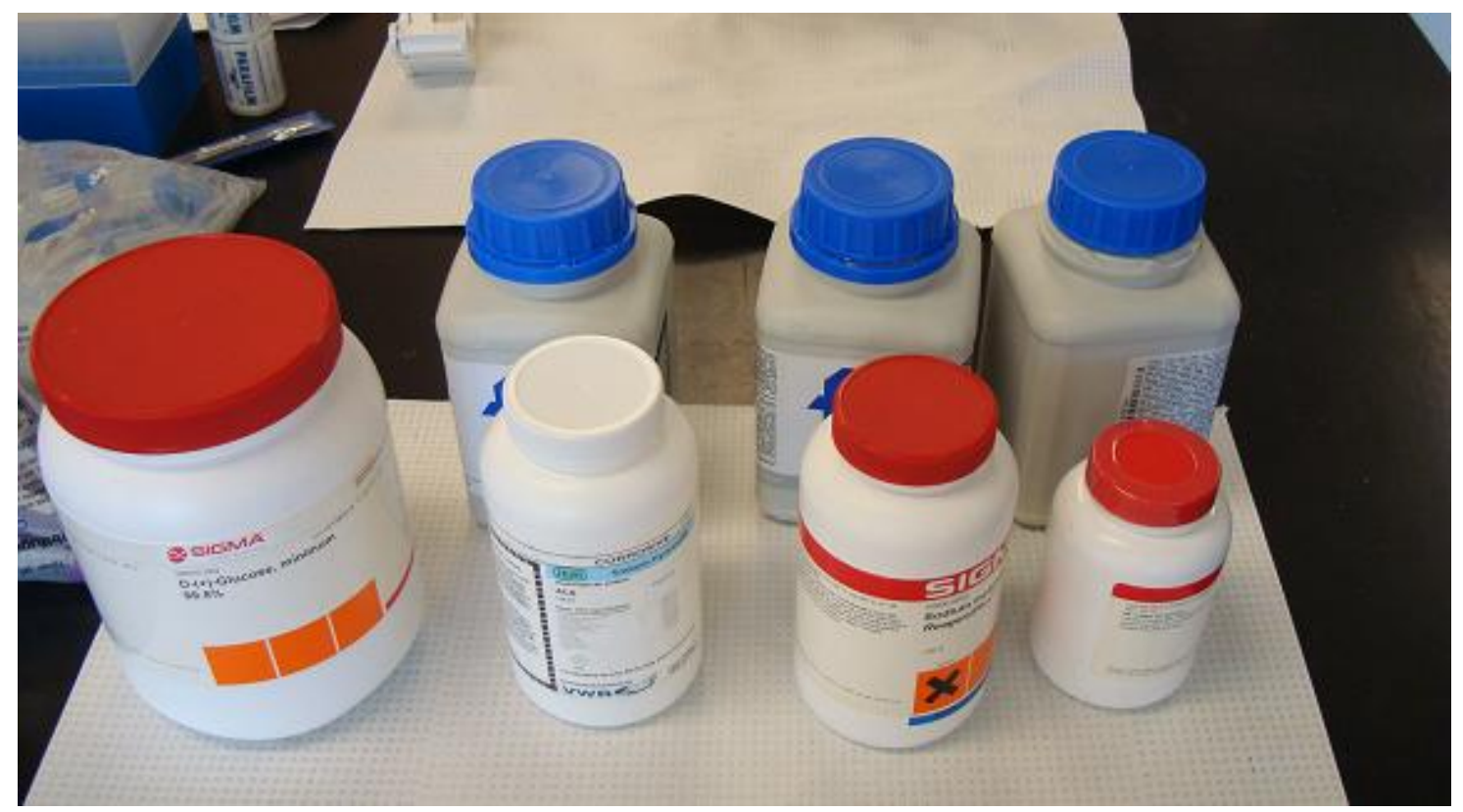

Picture 6: Chemicals used for tests 


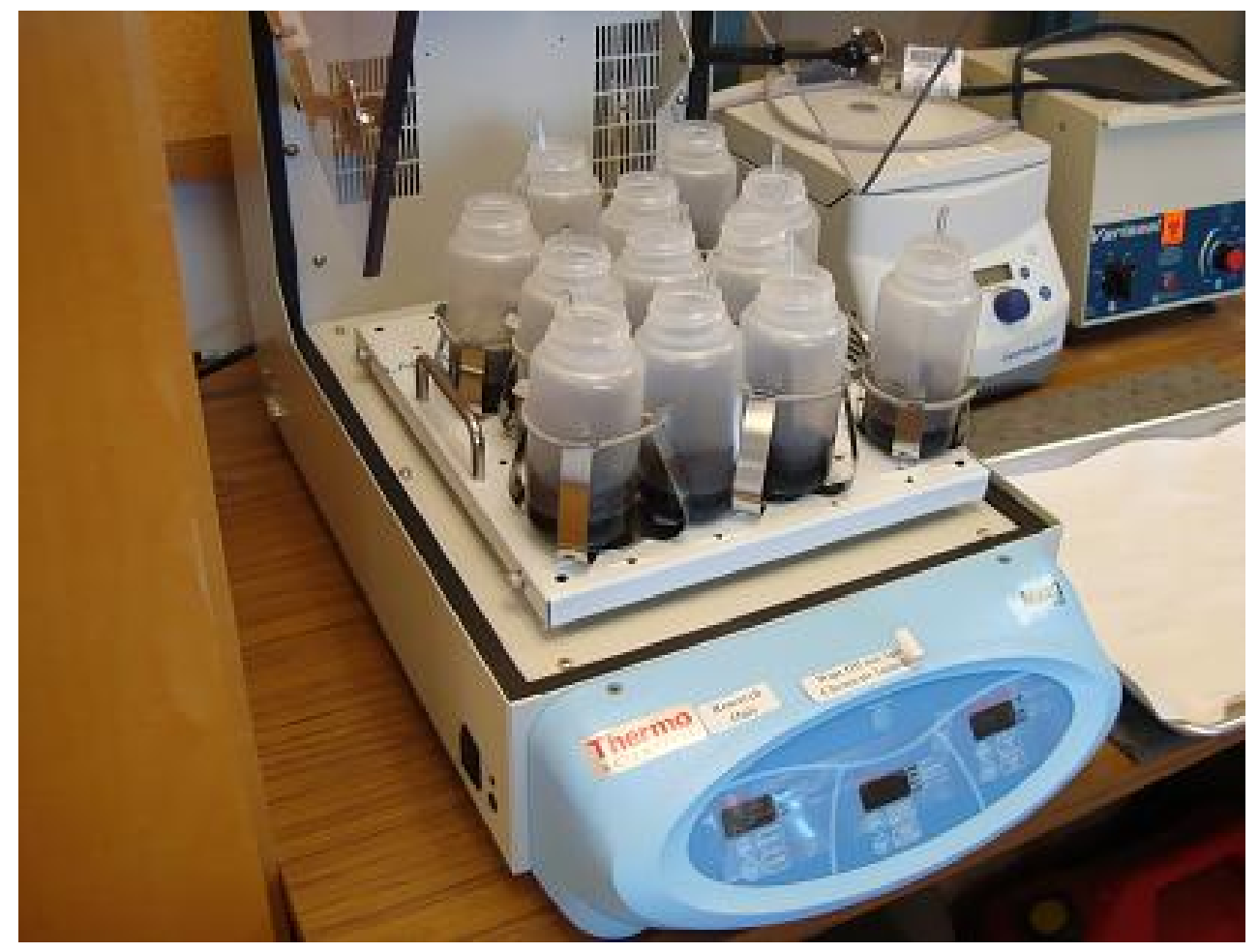

Picture 7: COSLIF pretreatment phase

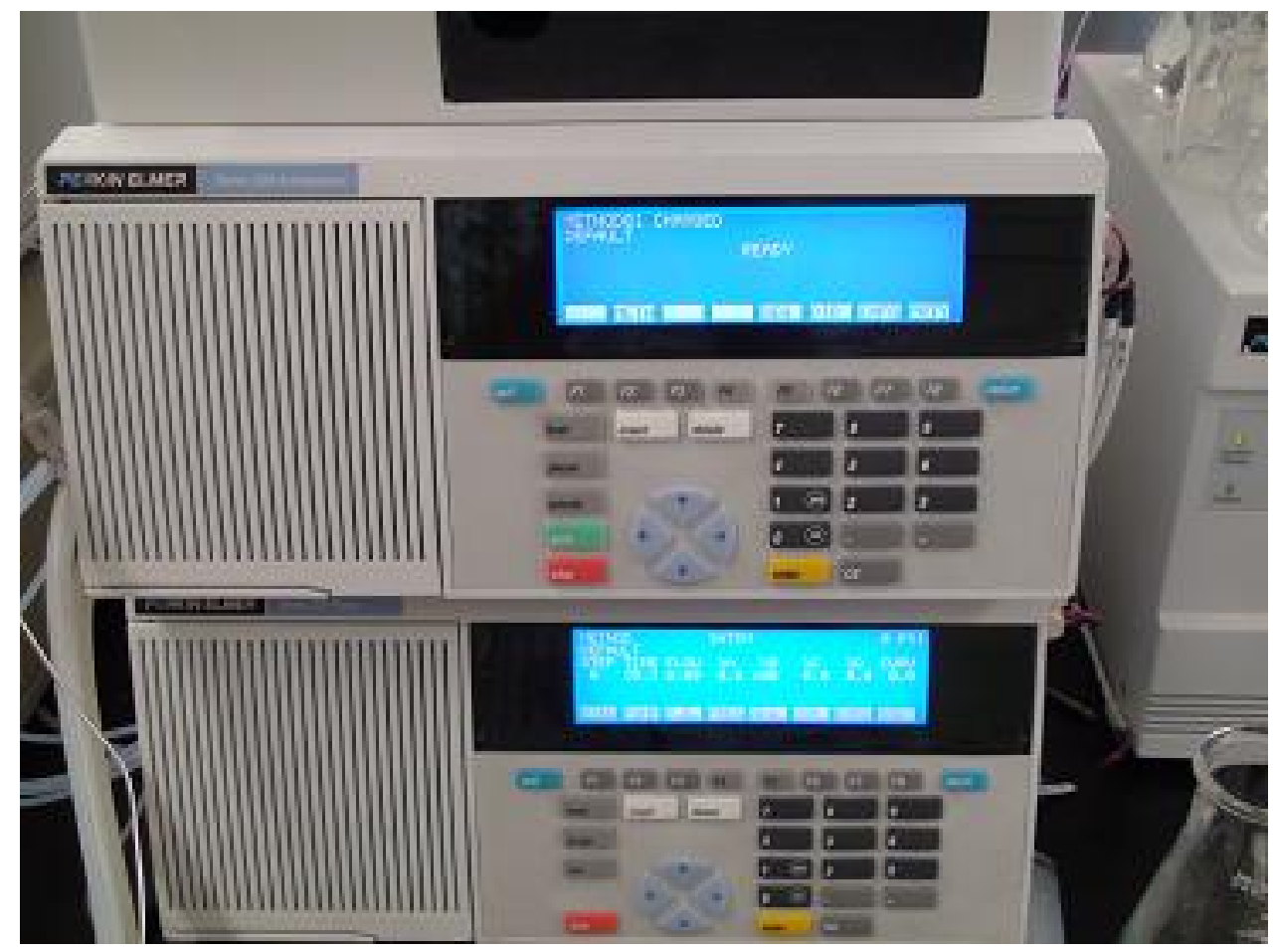

Picture 8: HPLC apparatus 


\section{APPENDIX B: ADSORPTION CAPACITY CALCULATIONS}

Adsorption capacity constant (Langmuir Isotherms)

Date: May 17, 2014

Table 1: Data parameters from experiment

\begin{tabular}{|c|c|c|c|c|c|}
\hline Samples & $\begin{array}{c}\text { Actual } \\
\text { Reading }\end{array}$ & Absorbance & $\overline{E_{\text {FREE }}}$ & Adsorbed & \% Adsorp \\
\hline Wat. Blank & 0 & & & & \\
\hline \multicolumn{6}{|l|}{ Subs. } \\
\hline Blank & 178 & 0.399 & & & \\
\hline $\mathrm{E}_{\mathrm{INIT}}$ & 395 & 0.859 & & & \\
\hline 1 & 288.3 & 0.635 & 110.3 & 284.7 & 72.07595 \\
\hline 2 & 293.3 & 0.646 & 115.3 & 279.7 & 70.81013 \\
\hline 3 & 282.9 & 0.623 & 104.9 & 290.1 & 73.44304 \\
\hline 4 & 295.1 & 0.65 & 117.1 & 277.9 & 70.35443 \\
\hline 5 & 293.3 & 0.646 & 115.3 & 279.7 & 70.81013 \\
\hline Avg. & & & & 282.42 & 71.49873 \\
\hline Std. Dev. & & & & & 1.26207 \\
\hline
\end{tabular}


Table 2: Data parameters calculation

\begin{tabular}{cccccc}
\hline Actual Reading & $E_{\text {Ads }}$ & $E_{\text {Free }}$ & $E_{\text {ads }} / E_{\text {free }}$ & $E e$ & $E_{\text {free }} / E e$ \\
\hline 282.9 & 290.1 & 104.9 & 2.765491 & 14.505 & 7.231989 \\
288.3 & 284.7 & 110.3 & 2.581142 & 14.235 & 7.748507 \\
293.3 & 279.7 & 115.3 & 2.425846 & 13.985 & 8.244548 \\
293.3 & 279.7 & 115.3 & 2.425846 & 13.985 & 8.244548 \\
295.1 & 277.9 & 117.1 & 2.373185 & 13.895 & 8.427492 \\
\hline
\end{tabular}

Amount of substrate in each test sample $=100 \mathrm{mg}$, SSO waste hydrolyzed

Wat.Blank = water blank

Subs.Blank $=$ substrate blank

E. Blank = Enzyme blank

Eads, $m$ = maximum Accellerase 1500 adsorbed ( $\mu$ g of cellulases/mg of substrate)

Kads $=$ adsorption constant $(\mu \mathrm{g} / \mathrm{ml})^{-1}$

$E e=$ Cellulases adsorbed, $\mu g$ Accellerase/ mg of substrate

Eads, $m$ and Kads are obtained from plot of the following Equation:

$\frac{E_{\text {in sol,ads }}}{E_{e}}=\frac{1}{K_{\text {ads }} \cdot E_{\text {ads }, m}}+\frac{1}{E_{\text {ads }}} \cdot E_{\text {in sol,ads }}$

Slope: 0.0979; Intercept: -3.0448 (Figure 1 below)

1/intercept: $0.328428797 ; \mathrm{K}_{\mathrm{Ads}}=0.003215318$ 


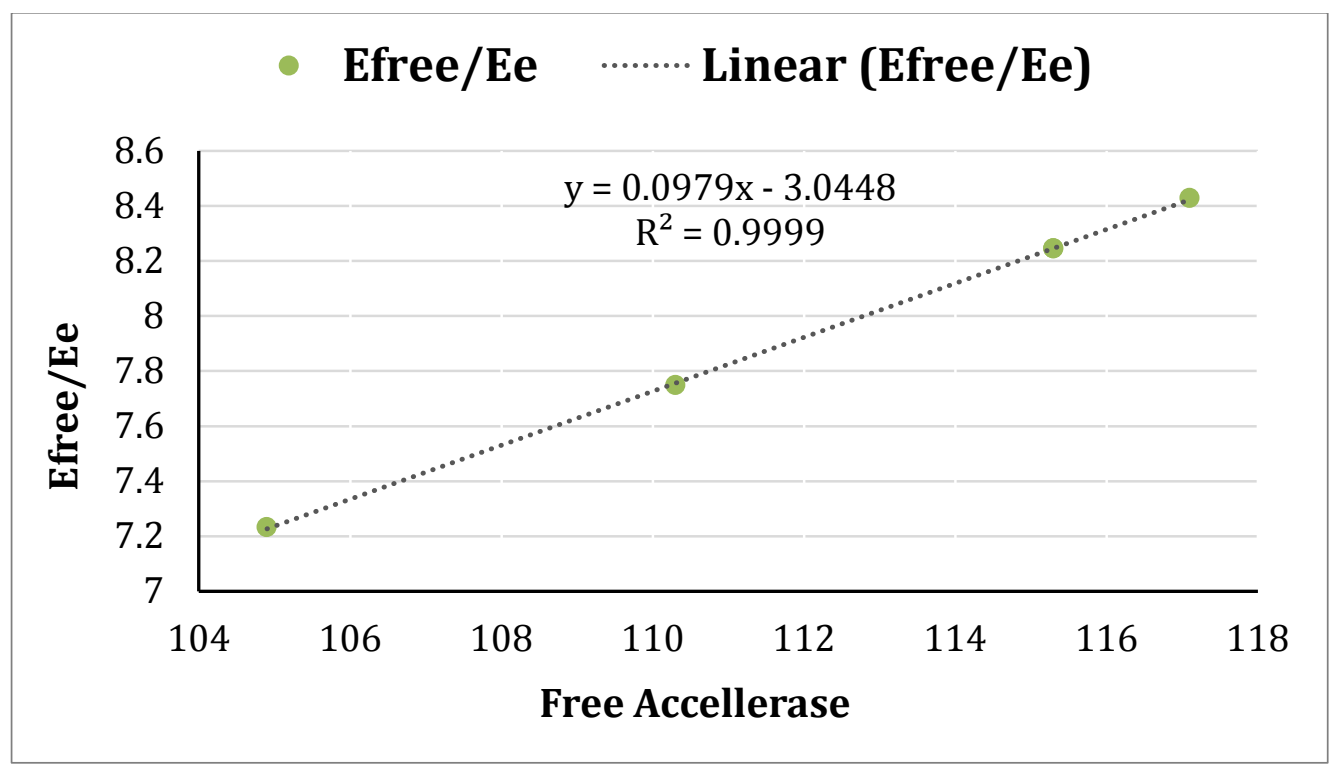

Figure 1: Langmuir isotherms plot 


\section{APPENDIX C: BERKELEY MADONNA COMPUTER PROGRAM CODE AND}

\section{TABULATED DATA}

Computer Program Codes and run for Kinetic Model on SSO

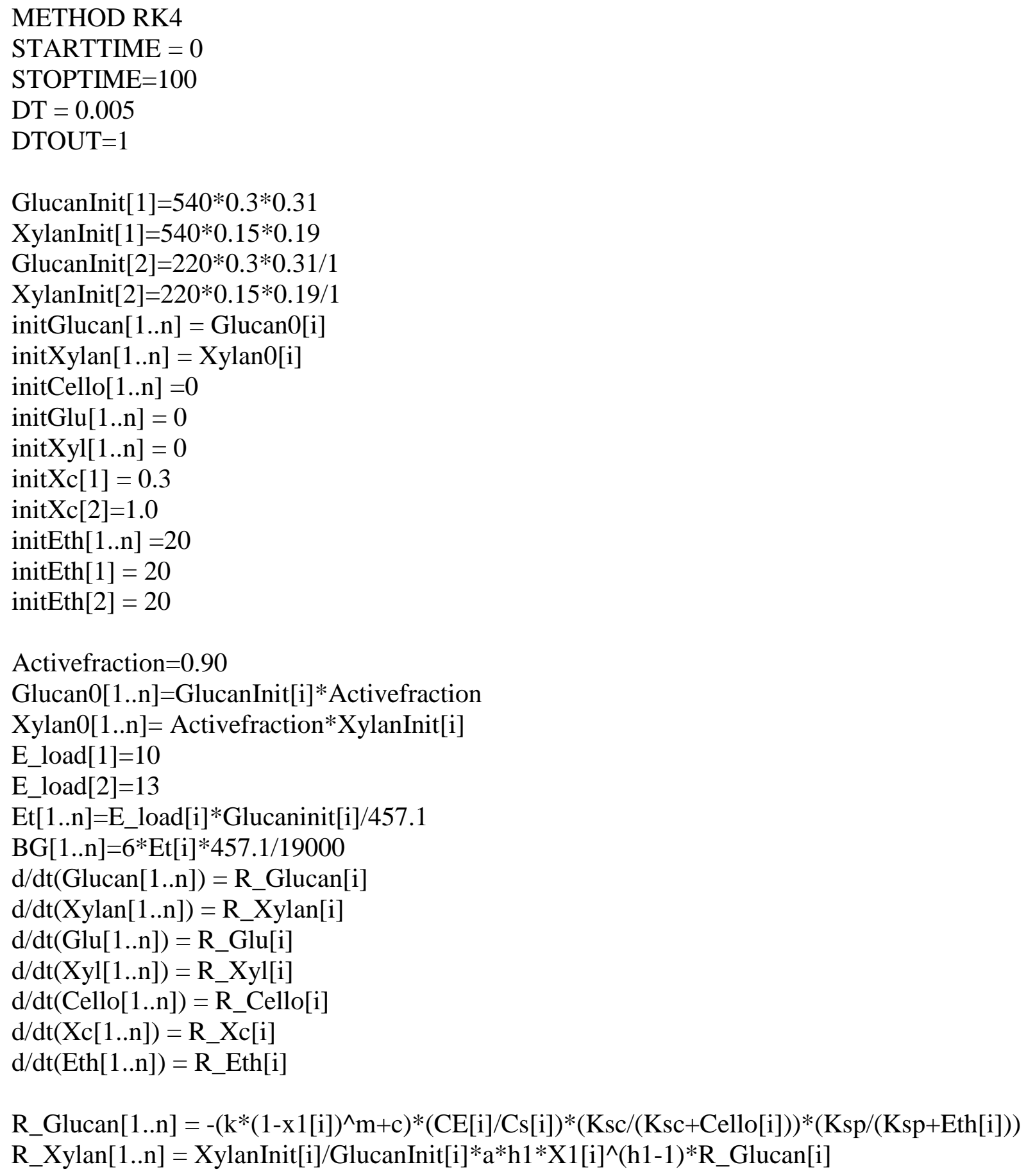


R_Glu[1..n] $=(-1.056 *$ R_Glucan[i]-R_cello[i])*1.053 - (u1[i]/YXGlumax+m1)*Xc[i]

R_Xyl[1..n] $=\left(-1.136 * R \_X y l a n[i]\right)-(u 2[i] / Y X X y l m a x+m 2) * X c[i]$

R_Cello[1..n] = -1.056*R_Glucan[i]-Kc*Cello[i]*BG[i]/(Km*(1+Glu[i]/Kcg)+Cello[i] $)$

R_Xc[1..n] $=(\mathrm{u} 1[\mathrm{i}]+\mathrm{u} 2[\mathrm{i}]-\mathrm{kd}-\mathrm{kde}[\mathrm{i}]) * \mathrm{Xc}[\mathrm{i}]$

R_Eth[1..n] $=(\mathrm{u} 1[\mathrm{i}] / \mathrm{YXGlumax}+\mathrm{m} 1) * \mathrm{Xc}[\mathrm{i}] * \mathrm{YEthGlu}+(\mathrm{u} 2[\mathrm{i}] / \mathrm{YXXylmax}+\mathrm{m} 2) * \mathrm{Xc}[\mathrm{i}]$

*YEthXyl

kde[1..n]=a1*exp(Cae/R*Eth[i])

$\mathrm{u} 1[1 . . \mathrm{n}]=\mathrm{Glu}[\mathrm{i}] /(\mathrm{kGlu}+\mathrm{Glu}[\mathrm{i}]+\mathrm{I} 1 * \mathrm{Xyl}[\mathrm{i}]) * \mathrm{U} 1 \mathrm{eth}[\mathrm{i}]$

U1eth[1..n] $=0.32 *(1-\text { Eth }[\mathrm{i}] / \text { Ethmax })^{\wedge} \mathrm{f} 1$

u2[1..n]=if U2eth[i] $>0$ then U2eth[i]*(Xyl[i]-XylT)/(kXyl*Xc[i]+Xyl[i]+I2*Glu[i]) else 0 U2eth[1..n]=Umax2*(1-Eth[i]/ETHMAX2)

$\mathrm{XylT}=\mathrm{kXyl} * \mathrm{~m} 2 * \mathrm{YXXylmax} / \mathrm{umax} 2$

$\mathrm{kd}=\mathrm{m} 1 *$ YXGlumax $+\mathrm{m} 2 *$ YXXylmax

$\mathrm{x} 1[1 . . \mathrm{n}]=($ Glucan0[i]-Glucan[i])/GlucanInit[i]

x2[1..n] $=($ Xylan0[i]-Xylan[i])/XylanInit[i]

GUESS E1 $=0.1$

ROOTS E1=Et[1]-E1-(Cs[1]-1)*Ks*E1*(Glucan[1]+Xylan[1])/(1+Ks*E1)

LIMIT E1 $>=0$

LIMIT E1<=Et[1]

$\mathrm{E}[1]=\mathrm{E} 1$

GUESS E2 $=0.1$

ROOTS E2=Et[2]-E2-(Cs[2]-1)*Ks*E2*(Glucan[2]+Xylan[2])/(1+Ks*E2)

LIMIT E2 $>=0$

LIMIT E2 $<=$ Et[2]

$\mathrm{E}[2]=\mathrm{E} 2$

$\mathrm{Cs}[1 . . \mathrm{n}]=\mathrm{Cs} 0$

$\mathrm{CE}[1 . . \mathrm{n}]=\mathrm{Ks} * \mathrm{Cs}[\mathrm{i}] * \mathrm{E}[\mathrm{i}] *(\mathrm{Glucan}[\mathrm{i}]+\mathrm{Xylan}[\mathrm{i}]) /(1+\mathrm{Ks} * \mathrm{E}[\mathrm{i}])$

$\mathrm{m} 1=0.034$

$\mathrm{m} 2=0.025$

umax1=0.32; $\{$ maximum growth rate from glucose, / h $\}$

umax2=0.2; $\{$ maximum growth rate from xylose, / h $\}$

YXGlumax $=0.08$

YXXylmax $=0.074$

a1=0.026; $\{$ Thermal ethanol death toxin coefficient, $/ \mathrm{h}\}$

Cae $=0.0037 * 8.314$; $\{$ Lipid-buffer partition coefficient, $\}$

$\mathrm{R}=8.314$;

Ethmax $=87.8$

ETHMAX2 $=62.3$

f1=2.93; \{ethanol inhibition factor to glucose consumption

YEthGlu=0.5; $\{$ ethanol tolerance for growth in glucose, $\mathrm{g} / \mathrm{g}$ \}

YEthXyl=0.46; $\{$ ethanol tolerance for growth in glucose, $g / g$ \}

KGlu=0.091; $\{$ Monod growth for glucose, g/L $\}$

KXyl=3.77; \{growth constant for xylose, dimensionless

I1=0.108; $\{$ Monod growth inhibitor from xylose, $g / L$ \} 
$\mathrm{I} 2=6.032 ;\{$ Monod growth inhibitor from glucose, $\mathrm{g} / \mathrm{L}\}$

$\mathrm{n}=2$; $\{$ number sets of data $\}$

$\mathrm{a}=1$

h1 $=1 ;\{$ xylan and glucan correlation constant, dimensionless $\}$

\{Hydrolysis kinetic parameters\}

$\mathrm{k}=0.69$; $\{$ Hydrolysis rate constant, $/ \mathrm{h}\}$

$\mathrm{m}=0.537$; $\{$ Exponent of the declining substrate reactivity, dimensionless $\}$

$\mathrm{c}=0.00 ;\{$ Conversion independent component in rate function, $/ \mathrm{h}$ \}

Ksc=5.85; $\{$ Inhibition of cellulose hydrolysis by cellibiose, $\mathrm{g} / \mathrm{L}\}$

$\mathrm{Ksp}=50.35$; $\{$ inhibition of cellulose hydrolysis by ethanol, g/L $\}$

$\mathrm{Cs} 0=1.29$;

$\mathrm{Cl}=1.0123$

Ks=0.466; $\{$ Adsorption constant for cellulosic fraction of biomass, $\mathrm{I} / \mathrm{U}\}$

$\mathrm{Kl}=0.807$

$\mathrm{kf1}=1.8366$

$\mathrm{kr} 1=\mathrm{kf} 1 / \mathrm{Ks}$

$\mathrm{kf} 2=0.8359$

$\mathrm{kr} 2=\mathrm{kf} 2 / \mathrm{Kl}$

$\mathrm{Kc}=663.4$

$\mathrm{Km}=10.56$

$\mathrm{Kcg}=0.62$; $\{$ Hydrolysis kinetic parameters from Xiongjun $\}$

$\mathrm{xc} 1=\mathrm{xc}[1]$

eth1=Eth[1]

Glu1=Glu[1]

Xyl1=Xyl[1]

Cello1=Cello[1]

$\mathrm{xc} 2=\mathrm{xc}[2]$

eth2=Eth[2]

Glu2=Glu[2]

$\mathrm{Xyl} 2=\mathrm{Xyl}[2]$

Cello2=Cello[2] 


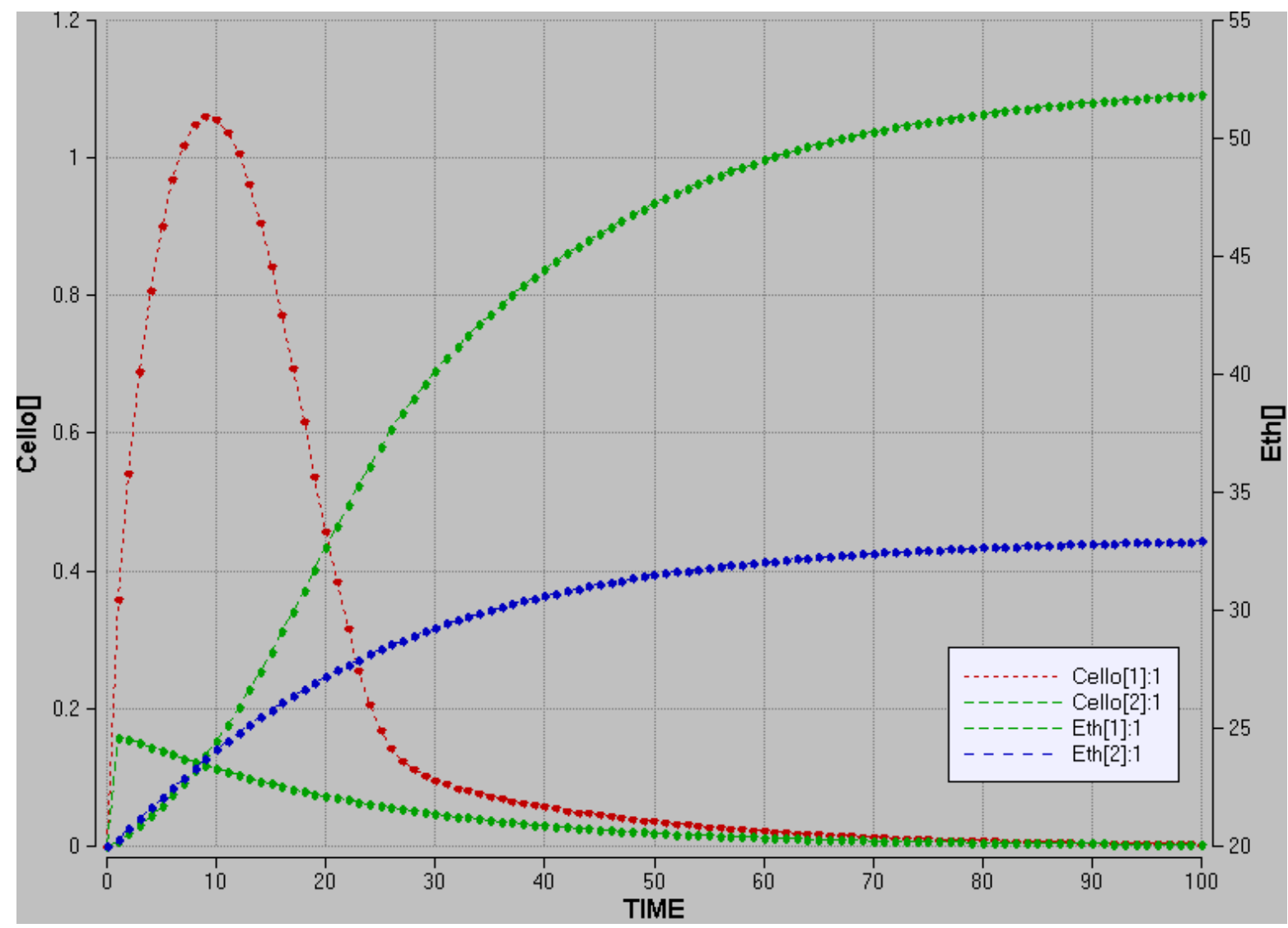

Figure 1: Kinetic model profiles (Cellomass vs Ethanol) 
Table 1: Berkeley Madonna program window parameters

\begin{tabular}{|c|c|}
\hline & \\
\hline ST ARTML & $\begin{array}{l}=0 \\
=100\end{array}$ \\
\hline SIOPTME & $=100$ \\
\hline DT & $=0.005$ \\
\hline DTOUT & $=1$ \\
\hline ROOTTOL & $=0.001$ \\
\hline Activefractio & $=0.9$ \\
\hline $\mathrm{m} 1$ & $=0.034$ \\
\hline m2 & $=0.025$ \\
\hline umax1 & $=0.32$ \\
\hline umax2 & $=0.2$ \\
\hline YXGlumax & $=0.08$ \\
\hline Yxxylmax & $=0.074$ \\
\hline a1 & $=0.026$ \\
\hline Cae & $=0.0307618$ \\
\hline $\mathrm{R}$ & $=8.314$ \\
\hline Ethmax & $=87.8$ \\
\hline ETHMAX2 & $=62.3$ \\
\hline f1 & $=2.93$ \\
\hline YEthGlu & $=0.5$ \\
\hline YEthXYI & $=0.46$ \\
\hline KGlu & $=0.091$ \\
\hline KXyl & $=3.77$ \\
\hline 11 & $=0.108$ \\
\hline 12 & $=6.032$ \\
\hline$n$ & $=2$ \\
\hline a & $=1$ \\
\hline h1 & $=1$ \\
\hline k & $=0.69$ \\
\hline $\mathrm{m}$ & $=0.537$ \\
\hline $\mathrm{c}$ & $=0$ \\
\hline $\mathrm{Ksc}$ & $=5.85$ \\
\hline Ksp & $=50.35$ \\
\hline $\mathrm{Cs} 0$ & $=1.29$ \\
\hline $\mathrm{Cl}$ & $=1.0123$ \\
\hline Ks & $=0.466$ \\
\hline KI & $=0.807$ \\
\hline kf1 & $=1.8366$ \\
\hline$k f 2$ & $=0.8359$ \\
\hline $\mathrm{Kc}$ & $=663.4$ \\
\hline
\end{tabular}


Table 2: Tabulated data from computer runs (Cellomass vs Ethanol)

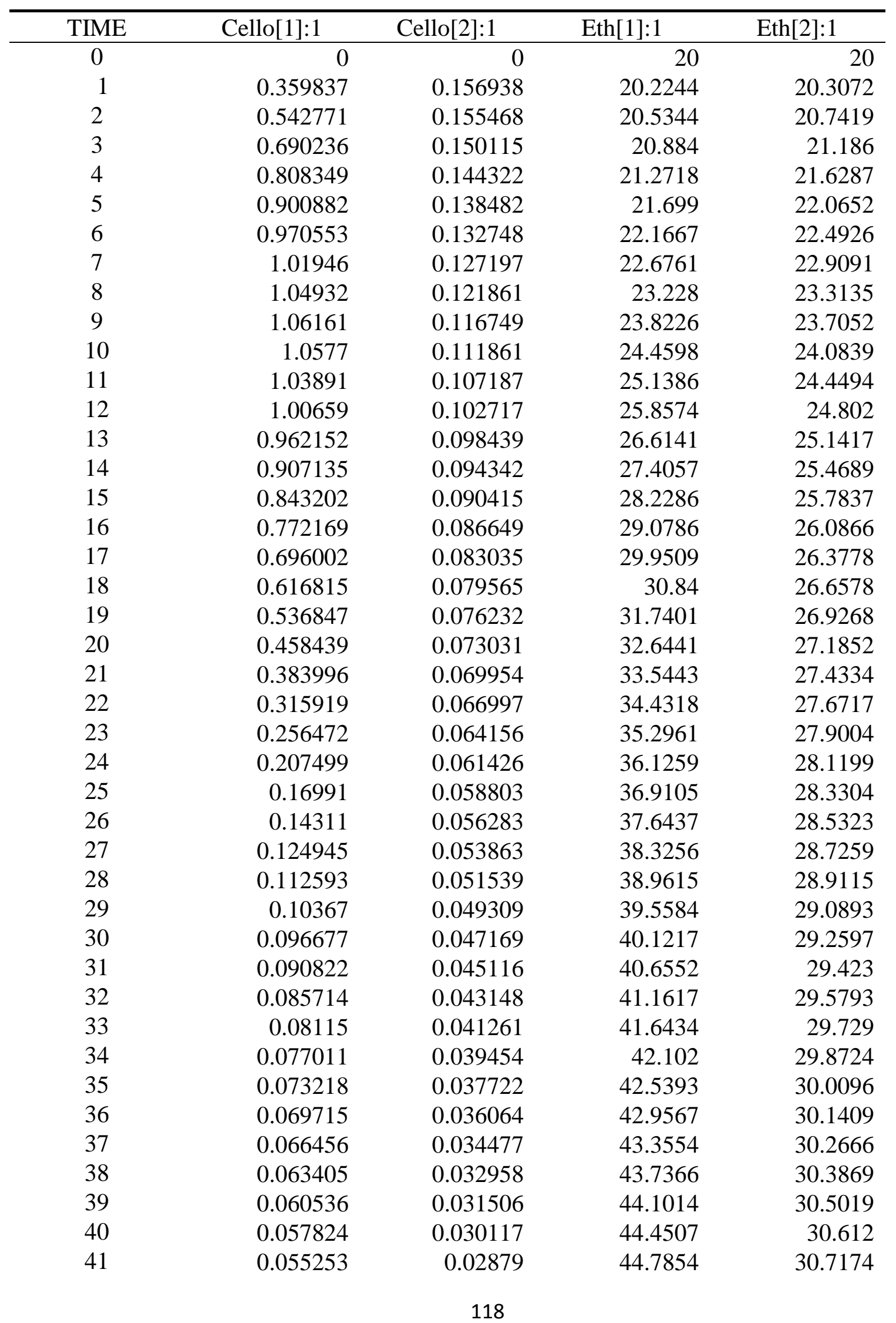




\begin{tabular}{|c|c|c|c|c|}
\hline TIME & Cello[1]:1 & Cello[2]:1 & Eth[1]:1 & Eth[2]:1 \\
\hline 42 & 0.052807 & 0.027521 & 45.1062 & 30.8181 \\
\hline 43 & 0.050474 & 0.026309 & 45.4139 & 30.9145 \\
\hline 44 & 0.048245 & 0.025151 & 45.7089 & 31.0067 \\
\hline 45 & 0.046113 & 0.024045 & 45.992 & 31.0949 \\
\hline 46 & 0.044069 & 0.02299 & 46.2635 & 31.1792 \\
\hline 47 & 0.04211 & 0.021982 & 46.5241 & 31.2599 \\
\hline 48 & 0.040231 & 0.02102 & 46.7742 & 31.3371 \\
\hline 49 & 0.038428 & 0.020103 & 47.0141 & 31.411 \\
\hline 50 & 0.036698 & 0.019227 & 47.2442 & 31.4816 \\
\hline 51 & 0.035037 & 0.018392 & 47.465 & 31.5492 \\
\hline 52 & 0.033444 & 0.017594 & 47.6768 & 31.6139 \\
\hline 53 & 0.031916 & 0.016834 & 47.8799 & 31.6758 \\
\hline 54 & 0.030452 & 0.016109 & 48.0747 & 31.735 \\
\hline 55 & 0.029048 & 0.015417 & 48.2615 & 31.7917 \\
\hline 56 & 0.027703 & 0.014757 & 48.4404 & 31.846 \\
\hline 57 & 0.026416 & 0.014127 & 48.612 & 31.8979 \\
\hline 58 & 0.025185 & 0.013527 & 48.7763 & 31.9477 \\
\hline 59 & 0.024007 & 0.012954 & 48.9338 & 31.9953 \\
\hline 60 & 0.022881 & 0.012407 & 49.0845 & 32.0409 \\
\hline 61 & 0.021805 & 0.011885 & 49.2289 & 32.0846 \\
\hline 62 & 0.020778 & 0.011388 & 49.3672 & 32.1265 \\
\hline 63 & 0.019797 & 0.010913 & 49.4995 & 32.1666 \\
\hline 64 & 0.018862 & 0.010459 & 49.6261 & 32.2051 \\
\hline 65 & 0.017969 & 0.010026 & 49.7473 & 32.2419 \\
\hline 66 & 0.017119 & 0.009613 & 49.8632 & 32.2772 \\
\hline 67 & 0.016308 & 0.009219 & 49.9741 & 32.3111 \\
\hline 68 & 0.015536 & 0.008842 & 50.0801 & 32.3435 \\
\hline 69 & 0.0148 & 0.008482 & 50.1815 & 32.3747 \\
\hline 70 & 0.014099 & 0.008138 & 50.2785 & 32.4045 \\
\hline 71 & 0.013432 & 0.00781 & 50.3711 & 32.4332 \\
\hline 72 & 0.012797 & 0.007496 & 50.4597 & 32.4607 \\
\hline 73 & 0.012193 & 0.007196 & 50.5444 & 32.4871 \\
\hline 74 & 0.011618 & 0.006909 & 50.6253 & 32.5124 \\
\hline 75 & 0.011071 & 0.006634 & 50.7026 & 32.5367 \\
\hline 76 & 0.01055 & 0.006372 & 50.7765 & 32.5601 \\
\hline 77 & 0.010054 & 0.006121 & 50.8471 & 32.5825 \\
\hline 78 & 0.009583 & 0.005881 & 50.9145 & 32.6041 \\
\hline 79 & 0.009135 & 0.005651 & 50.979 & 32.6248 \\
\hline 80 & 0.008708 & 0.005431 & 51.0405 & 32.6446 \\
\hline 81 & 0.008302 & 0.005221 & 51.0993 & 32.6637 \\
\hline 82 & 0.007915 & 0.005019 & 51.1554 & 32.6821 \\
\hline 83 & 0.007548 & 0.004826 & 51.2091 & 32.6998 \\
\hline 84 & 0.007198 & 0.004641 & 51.2603 & 32.7167 \\
\hline
\end{tabular}




\begin{tabular}{crrrr}
\hline TIME & Cello[1]:1 & Cello[2]:1 & Eth[1]:1 & \multicolumn{1}{c}{ Eth[2]:1 } \\
\hline 85 & 0.006865 & 0.004464 & 51.3092 & 32.733 \\
86 & 0.006548 & 0.004294 & 51.3559 & 32.7487 \\
87 & 0.006246 & 0.004131 & 51.4005 & 32.7638 \\
88 & 0.005959 & 0.003975 & 51.4431 & 32.7783 \\
89 & 0.005686 & 0.003825 & 51.4838 & 32.7923 \\
90 & 0.005426 & 0.003682 & 51.5227 & 32.8057 \\
91 & 0.005178 & 0.003544 & 51.5597 & 32.8187 \\
92 & 0.004942 & 0.003412 & 51.5952 & 32.8311 \\
93 & 0.004717 & 0.003285 & 51.629 & 32.8431 \\
94 & 0.004503 & 0.003164 & 51.6613 & 32.8546 \\
95 & 0.004299 & 0.003047 & 51.6922 & 32.8657 \\
96 & 0.004104 & 0.002935 & 51.7216 & 32.8764 \\
97 & 0.003919 & 0.002827 & 51.7498 & 32.8867 \\
98 & 0.003742 & 0.002723 & 51.7766 & 32.8967 \\
99 & 0.003574 & 0.002624 & 51.8023 & 32.9062 \\
100 & 0.003413 & 0.002528 & 51.8268 & 32.9154 \\
\hline
\end{tabular}




\section{APPENDIX D: EXPERIMENTAL PROCEDURES}

1. Quantitative saccharification (QS)

Materials/ Equipment Required:

- Sugars, high purity for standards $(98 \%+)$ - glucose, xylose, galactose, arabinose, mannose for examples

- $\mathrm{H}_{2} \mathrm{SO}_{4}, 72 \% \mathrm{w} / \mathrm{w}\left(12.00 \pm 0.02 \mathrm{M}\right.$ or specific gravity 1.6389 at $\left.15.6^{\circ} \mathrm{C}\right)$ - (RICCA R8191600-4A)

- Calcium carbonate, ACS grade (Fisher C64-500)

- DDW

- Glass stirring rod, 6”

- Serum glass bottles, crimp top style $125 \mathrm{~mL}$

- Crimp cap, aluminum (Fisher 0640614B)

- $\quad$ Rubber stoppers, blue (Fisher FSSP9717931)

- Crimper

- $\quad \mathrm{pH}$ paper for $\mathrm{pH}$ range between 0 and 14

- $\quad$ Syringe, sterile 3 mL (for sampling) - (Fisher B309657)

- $\quad$ Needle, sterile 23Gx1.5 (for sampling) - (Fisher 14-826-6C)

- $\quad$ Syringe filter, 0.22 um (for sampling) - (VWR CA28145-491)

- $\quad$ Microcentrifuge tubes, 2 mL (for sampling) - (Fisher 05-408-138)

- Autosampler vials with cap, 2 mL (for HPLC) - (Fisher 03378397)

- $\quad$ Pipette, disposable 10 mL (for pH adjustment) - (Fisher 07-200-574)

- $\quad$ Pipette pump 
- Erlenmeyer flasks, $50 \mathrm{~mL}$

- Convection ovens with temperature control to $45 \pm 3^{\circ} \mathrm{C}$ and $105 \pm 3^{\circ} \mathrm{C}$

- Autoclave capable of maintaining $121 \pm 3^{\circ} \mathrm{C}$ (Yamato SM300)

- Water bath set at $30 \pm 1^{\circ} \mathrm{C}$ (Grant OLS200)

- Benchtop centrifuge (for HPLC sample preparation) - (Eppendorf 5424)

- High Performance Liquid Chromatography (HPLC)-Perkin Elmer LC Autosampler, Series 200

- HPLC column, Bio-Rad Aminex7 HPX-87P

- Guard columns, cartridges appropriate for the column used

o Deashing guard column cartridges (\#125-0118), of the ionic form $\mathrm{H}^{+} / \mathrm{CO}_{3}$ from Bio-Rad, in series with cartridge holders (\#125-0131).

Procedure:

1. Oven dried sample at $45^{\circ} \mathrm{C}$.

2. Ensure particles of sample pass through 40 mesh screen by breaking up the solids during the drying process.

3. Place $0.3 \pm 0.01 \mathrm{~g}\left(\mathrm{~W}_{1}\right)$ of sample in $125 \mathrm{~mL}$ glass serum bottle (each sample must be run at least in duplicate).

4. Add $3.0 \pm 0.01 \mathrm{~mL}$ of $72 \% \mathrm{H}_{2} \mathrm{SO}_{4}$.

5. Mix for 1 minute with a glass stirring rod until sample is thoroughly wetted.

6. Place glass serum bottle in water bath at $30 \pm 1^{\circ} \mathrm{C}$ and hydrolyze for 1 hour.

7. Stir sample every 15 minutes to assure complete mixing and wetting. 
8. Dilute to a $4 \%$ acid concentration by adding $84.00 \pm 0.04 \mathrm{~mL}$ DDW. This can be done by using a scale.

9. Prepare sugar recovery standards (SRS) by placing $0.3 \mathrm{~g}$ of high purity sugar of interest (pre-dried at $45^{\circ} \mathrm{C}$ ) to the nearest $0.1 \mathrm{mg}$ into a $125 \mathrm{~mL}$ glass serum bottle (in duplicate). Add $10 \mathrm{~mL}$ of DDW and then add $348 \mathrm{uL}$ of $72 \% \mathrm{H}_{2} \mathrm{SO}_{4}$.

- The calculated sugar recovery standards (SRS) will be used to correct for losses due to the destruction of sugars during the hydrolysis process.

- $\quad \% \mathrm{SRS}=\frac{\text { conc. detectedby } H \mathrm{H} L \mathrm{C}, \frac{\mathrm{mg}}{\mathrm{mL}}}{\text { knownconc. of sugarbeforehydrolysis, } \frac{\mathrm{mg}}{\mathrm{mL}}} \times 100$

10. Stopper and crimp each bottle.

11. Mix the samples by inverting the serum bottles a few times to eliminate phase separation.

12. Autoclave the bottles for 1 hour at $121 \pm 3^{\circ} \mathrm{C}$ (liquid cycle only).

13. Allow bottles to cool for 20 minutes at room temperature before removing the seals and stoppers.

14. Transfer $20 \mathrm{~mL}$ supernatant of each hydrozate to $50 \mathrm{~mL}$ Erlenmeyer flasks.

15. Neutralize each flask to between $\mathrm{pH} 5$ and $\mathrm{pH} 6$ by adding calcium carbonate slowly with frequent swirling to avoid foaming. Check $\mathrm{pH}$ with $\mathrm{pH}$ paper to avoid over neutralization.

16. Filter each neutralized hydrolyzate using $3 \mathrm{~mL}$ syringe with 0.2 um filter. One portion goes into a Safe-Lock $1.5 \mathrm{~mL}$ microcentrifuge tube for fridge storage in case a repeat analysis is required (no more than 2 weeks in storage); the other portion goes directly into autosampler vial if dilution is not required. Dilution is required when the concentration is out of the calibration standard range. 
17. Prepare sugar calibration standards for each sugar of interest or a set of multi-component standards containing glucose, xylose, arabinose in the range of 0.2 to $12.0 \mathrm{mg} / \mathrm{mL}$ using HPX-87P column.

18. Instrumental conditions for HPX-87P column:

- Sample volume: 50uL.

- Eluant: 0.2 um filtered and degassed, deionized water.

- Flow rate: $0.6 \mathrm{~mL} / \mathrm{min}$.

- Column temperature: $85^{\circ} \mathrm{C}$.

- Detector: refractive index.

- Run time: 15 minutes data collection plus a 75 minute pump ramp.

o Note: Check test sample chromatograms. Levels of cellobiose greater than $3 \mathrm{mg} / \mathrm{mL}$ indicate incomplete hydrolysis and peaks appear before cellobiose may indicate sugar degradations.

o Details of procedure for HPLC sample preparation are in the fermentation section.

2. Cellulase activities

Cellulase activity is measured in terms of Filter Paper Units (FPU) per milliliter of original (undiluted) enzyme solution. The value of $2.0 \mathrm{mg}$ of reducing sugar as glucose from $50 \mathrm{mg}$ of filter paper (4\% conversion) in 60 minutes has been designated as the intercept for calculating Filter Paper cellulase Units (FPU). 
Materials/ Equipment Required:

- DDW (distilled de-ionized water)

- 2.65 g - 3,5 Dinitrosalicylic acid (Sigma 128848-100G)

- $4.95 \mathrm{~g}$ - Sodium hydroxide (Fisher S318-500)

- $76.5 \mathrm{~g}$ - Rochelle salts (sodium potassium tartrate) - (Fisher S387-500)

- $1.9 \mathrm{~mL}$ - Liquified Phenol (melt at 50 $\mathrm{C}$ ) - (Sigma P9346-500ML)

- 2.075 g - Sodium metabisulfite (Sigma S9000-500G)

- $210 \mathrm{~g}$ - Citrate acid monohydrate (Fisher A104-500)

- $\quad 0.1$ N HCl (Fisher 351280-500)

- Anhydrous glucose (Sigma G8270-1KG)

- Accellerase 1500

- Whatman No. 1 filter paper

- 5 - Test tube, plastic $50 \mathrm{~mL}$ (for enzyme dilution) - ( VWR 82018-050)

- 30 - Test tube, plastic $15 \mathrm{~mL}$ (26 for samples and 4 for glucose dilution) - (VWR 21008216)

- Test tube racks

- 26 - Cuvette w/ Cap (VWR 97000-584 and 89000-628)

- 1 - Graduated cylinder, $50 \mathrm{~mL}$ (for 1:20 enzyme dilution)

- 1 - Beaker, $250 \mathrm{~mL}$ (for DNS reagent)

- 1 - Beaker, 1 L (for Citrate buffer)

- 2 - Volumetric flask, $100 \mathrm{~mL}$ (for citrate buffer and glucose stock solution)

- Hot plate 
- Water bath capable of maintaining $50^{\circ} \mathrm{C} \pm 0.1^{\circ} \mathrm{C}$ (Grant OLS200)

- Spectrophotometer suitable for measuring absorbance at $540 \mathrm{~nm}$

Procedure for DNS reagent:

1. Mix and dissolve:

- $1416 \mathrm{~mL}-\mathrm{DDW}$

- 10.6 g - 3,5 Dinitrosalicylic acid

- $19.8 \mathrm{~g}$ - Sodium hydroxide

2. Add:

- $306 \mathrm{~g}$ - Rochelle salts (sodium potassium tartrate)

- $7.6 \mathrm{~mL}$ - Phenol (melt at $50^{\circ} \mathrm{C}$ )

- $8.3 \mathrm{~g}$ - Sodium metabisulfite

3. Take $3 \mathrm{ml}$ from the above mix and titrate with $0.1 \mathrm{~N} \mathrm{HCl}$ to the phenolphthalein endpoint. It should take 5-6 mL of $\mathrm{HCl}$.

4. Add $\mathrm{NaOH}$ to the mix if it takes less than $5-6 \mathrm{ml}$ of $\mathrm{HCl}$. No need to add if it is more (2g $=1 \mathrm{~mL} 0.1 \mathrm{~N} \mathrm{HCl})$.

Note:

- Make enough DNS reagent to be used. Avoid large batch that requires storage because of oxidation of sodium sulfite (Miller, 1958).

- Example (use only $1 / 10$ of the above amounts):

- $141.6 \mathrm{~mL}-\mathrm{DDW}$

- 1.06 g - 3,5 Dinitrosalicylic acid 
- $1.98 \mathrm{~g}$ - Sodium hydroxide

- $30.6 \mathrm{~g}$ - Rochelle salts (sodium potassium tartrate)

- $0.76 \mathrm{~mL}-$ Phenol

- $0.83 \mathrm{~g}$ - Sodium metabisulfite

Procedure for 1 M Citrate Buffer:

1. Mix and dissolve in $100 \mathrm{~mL}$ volumetric flask to make $1 \mathrm{M}$ stock (mix Citric acid to DDW first before adding $\mathrm{NaOH}$ to avoid violent chemical reaction):

- 21 g-Citric acid monohydrate

- $75 \mathrm{~mL}-\mathrm{DDW}$

- 5 to $6 \mathrm{~g}-\mathrm{NaOH}$ (add until $\mathrm{pH}=3$ )

2. Dilute to $100 \mathrm{~mL}$ and check $\mathrm{pH}$

3. Add $\mathrm{NaOH}$ until $\mathrm{pH}=4.5$ if necessary

4. Dilute the $1 \mathrm{M}$ citrate buffer stock to $0.05 \mathrm{M}$ (make $1 \mathrm{~L}$ )

5. Check the $\mathrm{pH}$ (should be 4.8). Adjust the $\mathrm{pH}$ to 4.8 if necessary

Procedure for Filter Paper Assay (Accelerase 1500):

1. Prepare $50 \mathrm{mg}$ (triplicate- 15 samples) Whatman No. 1 filter paper strip $(1.0$ x $6.0 \mathrm{~cm})$;

2. Place a rolled filter paper strip into each $15 \mathrm{~mL}$ test tube (plastic).

3. Add $1.0 \mathrm{~mL} 0.05 \mathrm{M}$ citrate buffer, $\mathrm{pH} 4.8$ to each tube; the buffer should saturate the filter paper strip.

4. Bring the tubes with their contents to $50^{\circ} \mathrm{C}$ in water bath. 
5. Add $0.5 \mathrm{ml}$ enzyme diluted appropriately in citrate buffer to each tube (enzyme dilutions are made in citrate buffer from stock solution that has been diluted 1:20 in citrate buffer).

6. Incubate tubes at $50^{\circ} \mathrm{C}$ for exactly 60 minutes.

7. Remove tubes from incubator.

8. Stop the reaction by immediately adding and mixing $3.0 \mathrm{~mL}$ DNS reagent to each tube.

9. The above procedure applies to blanks, controls and glucose standards (see note below).

10. Boil all tubes for exactly 5.0 minutes in boiling water bath (ensure contents in tubes are below the water level).

11. Transfer tubes to ice cold water.

12. Allow pulp to settle in each tube.

13. Dilute each tube in DDW in a cuvette $(0.2 \mathrm{~mL}$ of color-developed reaction mixture plus $2.5 \mathrm{~mL}$ of DDW).

14. Mix well.

15. Determine absorbance against reagent blank at $540 \mathrm{~nm}$.

16. Plot glucose standard curve. Glucose amounts $(\mathrm{mg} / 0.5 \mathrm{~mL})$ on $\mathrm{x}$-axis vs. $\mathrm{A}_{540}$ on $\mathrm{y}$-axis

17. Determine glucose released for each sample using the standard curve.

18. Plot glucose released $(\mathrm{mg} / 0.5 \mathrm{~mL})$ on $\mathrm{x}$-axis versus enzyme concentration on $\mathrm{y}$-axis using semi logarithmic graph paper.

19. Estimate enzyme concentration which would have release $2.0 \mathrm{mg}$ of glucose by drawing a line through two data points that are closest to $2.0 \mathrm{mg}$ on the $\mathrm{x}$-axis. Use this line to find the enzyme concentration that would produce exactly $2.0 \mathrm{mg}$ of glucose.

20. Input the estimated enzyme concentration in the following equation to find FPU/mL: 
o Filter Paper Activity $=\frac{0.37}{\text { Enzymeconcentrationreleasing } 2.0 \mathrm{mgglucose}}$ units $/ \mathrm{mL}$

o Note: Enzyme concentration refers to the number of $\mathrm{mL}$ of the original solution present in each $\mathrm{mL}$ of the dilution.

Note:

- $\quad$ Reagent blank $=1.5 \mathrm{~mL}$ citrate buffer.

- $\quad$ Enzyme control = 1.0 mL citrate buffer + $0.5 \mathrm{~mL}$ enzyme dilution (one for each dilution).

- $\quad$ Substrate control $=1.5 \mathrm{~mL}$ citrate buffer + filter paper strip.

- Glucose standards:

o Make up working stock of anhydrous glucose $(10 \mathrm{mg} / \mathrm{mL})$.

o Dilutions are made as follows:

\begin{tabular}{ccccc}
\hline $\begin{array}{c}\text { Glucose Stock } \\
(\mathrm{mL})\end{array}$ & $\begin{array}{c}\text { Citrate buffer } \\
(\mathrm{mL})\end{array}$ & Dilution & Concentration & $\begin{array}{c}\mathrm{A}_{540} \\
(\mathrm{~nm})\end{array}$ \\
\hline 1.0 & 0.5 & $1: 1.5$ & $3.35 \mathrm{mg} / 0.5 \mathrm{~mL}$ & \\
1.0 & 1.0 & $1: 2$ & $2.50 \mathrm{mg} / 0.5 \mathrm{~mL}$ \\
1.0 & 2.0 & $1: 3$ & $1.65 \mathrm{mg} / 0.5 \mathrm{~mL}$ \\
1.0 & 4.0 & $1: 5$ & $2.00 \mathrm{~g} / 0.5 \mathrm{~mL}$ & \\
\hline
\end{tabular}

- Add $0.5 \mathrm{~mL}$ of each of the dilution above to $1.0 \mathrm{~mL}$ of citrate buffer in a $15 \mathrm{~mL}$ test tube (plastic). 


\section{Modified COSLIF pretreatment}

In this study, 95\% (v/v) ethanol was used as the only organic solvent in the modified COSLIF pretreatment.

Materials/ Equipment Required:

- $55 \mathrm{~g}$ - SSO sample (for 11 samples of $5 \mathrm{~g}$ each with one as substrate blank)

- 11 - Sterilized centrifuge bottle, $250 \mathrm{~mL}$

- 1 - Beaker, 2 L (for supernatant)

- $\quad 1$ - Graduated cylinder, $50 \mathrm{~mL}$ (for measuring phosphoric acid)

- $\quad 2$ - Graduated cylinder, $200 \mathrm{~mL}$ (one for measuring ethanol and one for water)

- $440 \mathrm{~mL}$ - Phosphoric acid 85\% (Fisher A242-1)

- $3300 \mathrm{~mL}$ - Ethanol 95\% (Commercial Alcohols P016EA95)

- $4400 \mathrm{~mL}$ - DDW

- 11 - Glass stir rod, 6”

- Benchtop shaking incubator (ThermoMaxQ 4450)

- Centrifuge (Sorvall RC-5C PLUS)

Autoclave:

- 11 - Centrifuge bottle, $250 \mathrm{~mL}$

- $4400 \mathrm{~mL}$ - DDW

- 11 - Glass stir rod, 6” 
Procedure:

1. Weigh bottles and label them;

2. Place 5 grams of dry lignocellulosic sample in a $250 \mathrm{~mL}$ centrifuge bottle and then mix with $40 \mathrm{ml}$ of $85 \%$ concentrated phosphoric acid using a glass rod;

3. Incubate the solid/liquid slurry in the MaxQ 4450 benchtop shaking incubator at 150 rpm and $50^{\circ} \mathrm{C} \pm 0.2^{\circ} \mathrm{C}$ for 2 hours;

4. Add $100 \mathrm{ml}$ of ethanol into bottle and mix well by force to stop the biomass dissolution and weak hydrolysis reactions;

5. Centrifuge the bottle in a Sorvall RC-5C PLUS floor model centrifuge at $7000 \mathrm{rpm}$, room temperature for 15 minutes;

6. Decant the supernatant containing ethanol, acetic acid, phosphoric acid and lignin;

7. Add $200 \mathrm{~mL}$ of ethanol into the bottle containing the slurry of cellulose and hemicelluloses and mix well by force;

8. Repeat step 5 and step 6;

9. Add $200 \mathrm{~mL}$ of water into the bottle containing the solid pellet;

10. Centrifuge the bottle as in step 5;

11. Decant the supernatant containing ethanol and hemicelluloses;

12. Repeat step 9, 10 and 11 once more;

13. Weigh bottles with contents to determine the amount of water retained.

4. Enzymatic hydrolysis:

Materials/ Equipment Required:

- $10 \mathrm{~mL}$ - Filtered Accellerase 1500 
- $\mathrm{NH}_{4} \mathrm{OH}$ (for $\mathrm{pH}$ adjustment) - (EMD AX1303-6)

- $\quad \mathrm{HCl} 1 \mathrm{~N}$ (for pH adjustment) - (Fisher 351280-500)

- $\quad 70 \%$ ethanol spray bottle (for disinfection)

- $810 \mathrm{~mL}$ - Sterilized DDW

- 12 - Centrifuge bottle, $250 \mathrm{ml}$

- 1 - Graduated cylinder, $50 \mathrm{~mL}$ (for dilution of cellulosic pellets)

- 1 - Beaker, $250 \mathrm{~mL}$ (for $\mathrm{pH}$ meter)

- 2 - Pipette, disposable $10 \mathrm{~mL}$ (for $\mathrm{pH}$ adjustment) - (Fisher 07-200-574)

- 1 - Pipette pump

- Pipettor

- 1 box - Pipette tip, sterile 1000 uL (for sampling) - (VWR 89079-470)

- 45 - Syringe, sterile $3 \mathrm{~mL}$ (for sampling) - (Fisher B309657)

- 45 - Needle, sterile 23Gx1.5 (for sampling) - (Fisher 14-826-6C)

- 45 - Syringe filter, 0.22 um (for sampling) - (VWR CA28145-491)

- 45 - Microcentrifuge tubes, $2 \mathrm{~mL}$ (for sampling) - (Fisher 05-408-138)

- 1 - Stericup filter unit $0.22 \mathrm{um}, 250 \mathrm{~mL}$ (for Accelerase 1500) - (Fisher SCGVU02RE)

- Biological hood

- $\mathrm{pH}$ meter

- Benchtop shaking incubator (ThermoMaxQ 4450)

Autoclave:

- $810 \mathrm{~mL}$ - DDW 
- 1 box - Pipette tips, $1000 \mathrm{uL}$

- $50 \mathrm{~mL}$ graduated cylinder

Procedure - (modified for 10 samples +1 substrate blank +1 enzyme blank)

1. Procedure is to be carried out under a biological hood.

- Procedure for using the biological hood:

o Wipe the inside of the hood with $70 \%$ ethanol;

o Place everything including samples inside the hood (to avoid contamination);

o Turn on the UV light and the blower for 20 minutes;

o Turn off the UV light but keep the blower on.

2. Allow samples to thaw if frozen.

3. Using the results from QS, dilute the aqueous treated biomass in the $250 \mathrm{~mL}$ centrifuge bottle (after ethanol and water washes) to $20 \mathrm{~g}$ glucan/L (including the amount of enzyme) using sterilized DDW.

- Example:

o Average $1.5 \mathrm{~g}$ of glucose is found in the $5 \mathrm{~g}$ SSO samples (from ${ }^{*} \mathrm{QS}$ );

o $\quad$ Glucan $=0.9 \times 1.5 \mathrm{~g}=1.35 \mathrm{~g}$

o $\mathrm{mL}$ DDW to make $20 \mathrm{~g} / \mathrm{L}$ glucan $=\frac{1.35 \mathrm{~g}}{20 \mathrm{~g} / \mathrm{L}} \times 1000=67.5 \mathrm{~mL}$

${ }^{*} \mathrm{QS}$ - quantitative saccharification procedure

4. Shake well.

5. Adjust the $\mathrm{pH}$ value to 4.8 using $\mathrm{NH}_{4} \mathrm{OH}$ (as a source of nitrogen). 
6. Bring the contents of each bottle to $50^{\circ} \mathrm{C}$ (this step must be done prior to the addition of enzyme if the rate of enzymatic release of glucose is to be measured).

7. Add filtered Accellerase 1500 loading of 30 FPU per g glucan.

- Example:

o Filter paper unit $=53 \mathrm{FPU} / \mathrm{mL}$

$0 \quad$ Glucan $=1.35 \mathrm{~g}$

○ $\mathrm{mL}$ to make $30 \mathrm{FPU} / \mathrm{g}$ glucan $=\frac{30 \frac{F P U}{g} \times 1.35 \mathrm{~g}}{53 \mathrm{FPU} / \mathrm{mL}}=0.76 \mathrm{~mL}$

8. Incubate and shake sample at $50^{\circ} \mathrm{C} \pm 0.2^{\circ} \mathrm{C}$ and $250 \mathrm{rpm}$ for 72 hours (or until the amount of released glucose becomes negligible when measured by HPLC, Bio-Rad HPX-87P column).

If the rate of released glucose is to be measured:

1. Remove $1.5 \mathrm{~mL}$ aliquot from flask at $0,12,24,48$ and 72 ;

2. Expel sample into $2 \mathrm{~mL}$ microcentrifuge tube;

3. Centrifuge sample then filter it into autosampler vial using syringe filter 0.2um Analyze the supernatant for released (soluble) glucose using HPLC, Bio-Rad HPX-87P column).

Calculation for percent digestion:

1. Determine glucose concentration in the supernatant using HPLC, Bio-Rad HPX-87P column; 
2. Subtract the glucose concentration, if any, from the substrates and enzyme blanks (see note below);

3. Correct for hydration (multiply the glucose reading by 0.9 to correct for the water molecule added in the hydrolysis of the cellulose) and multiply by the total volume of assay.

- Example:

o Glucose concentration in the supernatant (corrected with blanks) $=9.9$ $\mathrm{mg} / \mathrm{mL}$;

o Total volume of essay $=10 \mathrm{~mL}$;

o Then the amount of cellulose digested $=0.0099 \mathrm{~g} / \mathrm{mL}$ x $10 \mathrm{~mL}$ x 0.9 $=0.0891 \mathrm{~g} ;$

4. $\%$ digestion $=\frac{\text { grams cellulose } \text { digested }}{\text { grams cellulose } \text { added }} \times 100$

Note:

- Substrate blank contains DDW, $\mathrm{NH}_{4} \mathrm{OH}$, substrate and identical amount of volume with no enzyme.

- Enzyme blank contains DDW, $\mathrm{NH}_{4} \mathrm{OH}$, enzyme and identical amount of volume with no substrate.

5. Fermentation

Materials/Equipment Required:

- 2.5 g - Yeast extract (Fisher BP1422-500)

- $0.5 \mathrm{~g}$ - Potassium phosphate monobasic $\left(\mathrm{KH}_{2} \mathrm{PO}_{4}\right)$ - (EMD PX1565-1) 
- 25 g - Glucose (Sigma G8270-1KG)

- 5 g - Xylose (Fisher X1500-500G)

- $\mathrm{NH}_{4} \mathrm{OH}$ (for $\mathrm{pH}$ adjustment) - (EMD AX1303-6)

- $\quad \mathrm{HCl} 1 \mathrm{~N}$ (for $\mathrm{pH}$ adjustment) - (Fisher 351280-500)

- $1 \mathrm{~L}-\mathrm{DDW}$

- $5 \mathrm{~mL}-40 \%$ glycerol

- $70 \%$ ethanol spray bottle

- Z. mobilis 8b cell stock

- Pipettor

- 2 boxes - Pipette tip

- $\quad$ Pipette pump

- 3 - Pipette, disposable $10 \mathrm{~mL}$ (for $\mathrm{pH}$ adjustment and $10 \%$ transfer in fermentation procedure) - (Fisher 07-200-574)

- 1 - Graduated cylinder, $50 \mathrm{~mL}$ (for 10X RM rich media)

- 1 - Graduated cylinder, $250 \mathrm{~mL}$ (for 10:2 RMGX media)

- 1 - Volumetric flask, $250 \mathrm{~mL}$ (for 10:2 RMGX media)

- 1 - Glass stir rod, 8” (for mixing in rich media)

- 3 - Graduated cylinder, $100 \mathrm{~mL}$ (for seeding and preparing blanks)

- 1 - Beaker, $250 \mathrm{~mL}$ (for $\mathrm{pH}$ meter)

- 15 - Stericup filter unit $0.22 \mathrm{um}, 250 \mathrm{~mL}$ (1 for media and 14 for hydrolysate) - (Fisher SCGVU02RE)

- 15 - Test tube, plastic $15 \mathrm{~mL}$ (1 for pre-seed procedure and 14 for cell harvest) - (VWR 21008-216) 
- Test tube rack

- $\quad$ Cuvette w/ Cap (for checking OD) - (VWR 97000-584 and 89000-628)

- 15 - Serum glass bottles, crimp top style $125 \mathrm{~mL}$ (1 for seed procedure, 10 for hydrolysate, 1 for controlled glucose, 1 for DDW blank, 1 for substrate blank and 1 for enzyme blank)

- 15 - Crimp cap, aluminum (Fisher 0640614B)

- 15 - Rubber stoppers, blue (Fisher FSSP9717931)

- Crimper

- 10 - Cryovial 2 mL (VWR 16001-102)

- 100 - Syringe, sterile 3 mL (for sampling) - (Fisher B309657)

- 100 - Needle, sterile 23Gx1.5 (for sampling) - (Fisher 14-826-6C)

- 100 - Syringe filter, 0.22 um (for sampling) - (VWR CA28145-491)

- 100 - Microcentrifuge tubes, 2 mL (for sampling) - (Fisher 05-408-138)

- 100 - Autosampler vials with cap, 2 mL (for HPLC) - (Fisher 03378397)

- Biological hood

- Balance

- $\mathrm{pH}$ meter

- Vortex

- Spectrophotometer suitable for measuring absorbance at $540 \mathrm{~nm}$ (Eppendorf, BioPhotometer)

- Benchtop centrifuge (for cell harvest) - (Thermo Sorvall Legend RT+)

- Benchtop shaking incubator (ThermoMaxQ 4450)

- Benchtop centrifuge (for HPLC sample preparation) - (Eppendorf 5424) 
- HPLC-Perkin Elmer LC Autosampler, Series 200

- HPLC column, Bio-Rad Aminex7 HPX-87P (for glucose) and Bio-Rad Aminex7 HPX87H (for ethanol)

- Guard columns, cartridges appropriate for the column used

- For P column, use deashing guard column cartridges (\#125-0118), of the ionic form $\mathrm{H}^{+} / \mathrm{CO}_{3}$ from Bio-Rad, in series with cartridge holders (\#125-0131). For $\mathrm{H}$ column, use Cation H cartridge (\#125-0129) with holder (\#125-0131).

Autoclave:

- 1 - Graduated cylinder, $50 \mathrm{~mL}$ (for 10X RM rich media)

- 1 - Graduated cylinder, $250 \mathrm{~mL}$ (for 10:2 RMGX media)

- 1 - Volumetric flask, $250 \mathrm{~mL}$ (for 10:2 RMGX media)

- 3 - Graduated cylinder, $100 \mathrm{~mL}$ (for seeding and preparing blanks)

- 1 - Beaker, $250 \mathrm{~mL}$ (for $\mathrm{pH}$ meter)

- $\quad 3-100 \mathrm{~mL}$ graduated cylinder (for seeding and preparing blanks)

- 15 - Test tube, plastic $15 \mathrm{~mL}$ (1 for pre-seed procedure and 14 for cell harvest) - (VWR 21008-216)

- 1 - Glass stir rod, 8” (for mixing in rich media)

- 2 boxes - Pipette tip

- 15 - Rubber stoppers, blue (Fisher FSSP9717931)

- $10-40 \%$ Glyserol in cryovial

- $1 \mathrm{~L}-\mathrm{DDW}$ 
Procedure for Rich Media (10XRM):

- Yeast Extract: $100 \mathrm{~g} / \mathrm{L}$

- Potassium Phosphate Monobasic $\left(\mathrm{KH}_{2} \mathrm{PO}_{4}\right): 20 \mathrm{~g} / \mathrm{L}$

- DDW

- Sterilize with 0.2 um filter

Note:

- Make only $25 \mathrm{~mL}$ of 10X RM (add $2.5 \mathrm{~g}$ Yeast Extract and $0.5 \mathrm{~g} \mathrm{KH}_{2} \mathrm{PO}_{4}$ to $50 \mathrm{~mL}$ DDW).

- Gradually adding Yeast Extract and $\mathrm{KH}_{2} \mathrm{PO}_{4}$ to DDW and simultaneously shaking the graduated cylinder to ensure the solutes are completely dissolved.

Procedure for 10:2 RMGX Media (revive and intermediate seed):

- $1 \mathrm{X} \mathrm{RM}$

- Glucose: 100 g/L

- Xylose: 20 g/L

- DDW

- Sterilize with 0.2 um filter 
Note:

- Dilute 10X RM with DDW to make 1X RM (transfer $25 \mathrm{~mL}$ of 10X RM to $250 \mathrm{~mL}$ graduate cylinder and fill it with DDW to $250 \mathrm{~mL}$ mark).

- Add 25 g Glucose and 5 g Xylose to a $250 \mathrm{~mL}$ volumetric flask and fill it with $1 \mathrm{X}$ RM to marking).

Procedure for revive (pre-seed):

1. Remove the cell stock vial(s) from the freezer and allow it to gradually thaw at room temperature (at least 0.5 hour).

2. Vortex the cell stock vial(s) for 5 seconds.

3. Pipette (to prevent cells being stuck to vial wall) it into the revive media at a concentration of $10 \%$ using $15 \mathrm{~mL}$ centrifuge vial, that is, $1 \mathrm{ml}$ of cell stock into a $9 \mathrm{ml}$ of filter-sterilized (0.2 um filter) nutrient media (10:2 RMGX).

4. Incubate the culture at $33^{\circ} \mathrm{C}$ for approximately 8 hours.

5. Take a sample under biological hood and measure OD at $600 \mathrm{~nm}$ :

- $\quad$ Take $0.5 \mathrm{ml}$ of sample in a cuvette (allow bubbles to be released);

- $\quad$ Add $2.5 \mathrm{ml}$ sterile DDW;

- Mix well;

- $\quad$ Read and record OD at $600 \mathrm{~nm}$ (adjustment for dilution is required).

6. Calculate the volume needed to inoculate seed media (10:2 RMGX) at an OD of 0.01 in the procedure for seed;

- Example: 

o Recorded OD $=4$
o Required OD (for seed media) $=0.01$
o Working Volume (for seed media) $=100 \mathrm{ml}$
o $\quad C_{1} V_{1}=C_{2} V_{2}=>(4) V_{1}=(0.01)(100)=>V_{1}=0.25 \mathrm{ml}$

Procedure for seed:

1. Transfer calculated amount $(0.25 \mathrm{~mL})$ of revive culture to $125 \mathrm{~mL}$ serum bottle with crimp top containing $100 \mathrm{~mL}$ of seed media (10:2 RMGX);

2. Incubate the inoculated seed bottle in the shaking incubator at $150 \mathrm{rpm}$ and $33^{\circ} \mathrm{C}$ for 12 14 hours to reach an acceptable OD and glucose concentration;

3. Harvest the cell (stop the shaking incubator) when glucose concentration of the media reaches an acceptable OD (2.0 to 3.8) as determined from cell growth curves.

Procedure for restocking cells:

1. Mix sterile DDW with glycerol to a final concentration of $40 \%$;

Place 500 ul of $40 \%$ glycerol solution into each cryovial;

2. Autoclave this mixture with caps on but not shut tightly;

3. When they are cooled down, add 500 ul of an overnight culture (grown in growth media) to each cryovial;

4. Vortex thoroughly and store at $-80^{\circ} \mathrm{C}$. 
Procedure for Fermentation:

1. This procedure is to be carried out under the biological hood;

2. Create a controlled glucan (20 g/L) and blank (sterilized DDW), having the sample conditions as the hydrolysate;

3. Filter hydrolysate using $250 \mathrm{~mL}$ stericup;

4. Adjust $\mathrm{pH}$ of the hydrolysate to 6 using $\mathrm{NH}_{4} \mathrm{OH}$ and $1 \mathrm{~N} \mathrm{HCl}$;

5. Transfer the hydrolysate to $125 \mathrm{~mL}$ serum bottle and use a scale to determine the amount;

6. For each bottle, transfer $10 \%(\mathrm{v} / \mathrm{v})$ of the seed media to a $15 \mathrm{~mL}$ centrifuge tube and centrifuge at $5000 \mathrm{rcf}$ (or $3500 \mathrm{rpm}$ ) for 5 minutes at $4^{\circ} \mathrm{C}$. Decant the supernatant. Resuspend the cell pellet with the hydrolysate by vortex. Transfer the volume back into the 125 mL serum bottle;

7. Crimp all serum bottles;

8. Flush all serum bottles with nitrogen;

9. Incubate all $125 \mathrm{~mL}$ serum bottles in the shaking incubator at $250 \mathrm{rpm}$ and $33^{\circ} \mathrm{C}$ for 24 hours;

10. Take samples to be analyzed for sugars and ethanol concentration by HPLC at $0,6,12$, 24 and 48 hour;

11. Calculate the \% theoretical ethanol yield by using the following formula:

$$
\% \text { theoretical ethanol yield }=\frac{[\text { EtOH }] f-[\text { EtOH }] i}{0.51(f[\text { Biomass }] 1.111)} \times 100
$$

where $[\mathrm{EtOH}]_{\mathrm{f}}$ - ethanol conc. at the end of fermentation, (g/L); $[\mathrm{EtOH}] \mathrm{i}$ - ethanol conc. at the beginning of fermentation, (g/L); [Biomass] - dry biomass conc. at the beginning of fermentation, (g/L); f - Cellulose fraction of dry biomass, (g/g); 0.51 - Conversion factor for glucose to ethanol; 1.111 -conversion factor for cellulose to equivalent glucose 
Procedure for HPLC Sample Preparation:

1. Mobile phase must be prepared fresh for each run. The first two HPLC vials are buffer containing only mobile phase contents.

2. Prepare duplicate standards for each run.

3. Centrifuge sample in $2 \mathrm{~mL}$ centrifuge tubes at $10,000 \mathrm{rcf}$ for 5 minutes to remove protein and particulates, then filter ( $0.22 \mathrm{um})$ about $1 \mathrm{~mL}$ of the supernatant into HPLC vial. Sample can be stored in the fridge for a few days if not used immediately.

4. Instrumental conditions for HPX-87P column:

- Sample volume: 50uL.

- Eluant: 0.2 um filtered and degassed, deionized water.

- Flow rate: $0.6 \mathrm{~mL} / \mathrm{min}$.

- Column temperature: $85^{\circ} \mathrm{C}$.

- Detector: refractive index.

- Run time: 15 minutes data collection plus a 75 minute pump ramp.

5. Instrumental conditions for HPX-87H column:

- Sample volume: 50uL.

- Eluant: 0.2 um filtered and degassed, $0.005 \mathrm{M}$ sulfuric acid.

- Flow rate: $0.6 \mathrm{~mL} / \mathrm{min}$.

- Column temperature: $65^{\circ} \mathrm{C}$.

- Detector: refractive index.

- Run time: 25 minutes data collection plus a 75 minute pump ramp. 


\section{APPENDIX E: HPLC SAMPLES RUNS FOR ENZYMATIC HYDROLYSIS}

AND FERMENTATION 


$\begin{array}{llll}\text { Software Version } & : 6.3 .2 .0646 & \text { Date } & : 6 / 10 / 2013 \text { 6:26:57 PM } \\ \text { Operator } & : \text { rLuong } & \text { Sample Name } & : \text { S1 } \\ \text { Sample Number } & : \text { O08 } & \text { Study } & : \text { Glucose } \\ \text { AutoSampler } & : \text { SER200 } & \text { Rack/Vial } & : 1 / 8 \\ \text { Instrument Name } & : \text { HPLC } & \text { Channel } & : \text { A } \\ \text { Instrument Serial \# } & : \text { None } & \text { A/D mV Range }: 1000 \\ \text { Delay Time } & : 0.00 \mathrm{~min} & \text { End Time } & : 14.99 \mathrm{~min} \\ \text { Sampling Rate } & : 2.5000 \mathrm{pts} / \mathrm{s} & & \\ \text { Sample Volume } & : 1.000000 \mathrm{ul} & & \\ \text { Sample Amount } & : 1.0000 & \text { Area Reject } & : 0.00000 \\ \text { Data Acquisition Time } & : 6 / 7 / 2013 \text { 3:28:05 PM } & \text { Dilution Factor }: 1.00\end{array}$

Raw Data File : C:IHPLC Datalrobin June 7-13_GlucoselEH_Glucose_June 7008.raw Result File : c:Ihplc datalrobinljune 7-13_glucoseleh_glucose_june 7008.rst [Editing in Progress] Inst Method : C:IHPLC DatalrobinlMethod Robin 87P from C:IHPLC DatalrobinlJune 7-13 GlucoselEH Glucose June 7008.raw Proc Method : C:IHPLC DatalrobinlMethod Robin 87P from c:Ihplc datalrobinljune 7-13_glucoseleh_glucose_june 7008.rst [Editing in Progress] Calib Method : C:IHPLC DatalrobinlMethod Robin 87P from c:Ihplc datalrobinljune 7-13_glucoseleh_glucose_june 7008.rst [Editing in Progress] Report Format File: C:IHPLC DatalrobinlMethod Robin 87P.rpt

Sequence File : C:IHPLC DatalrobinIValera Sequence 87p-glucose-June 7-13.seq

\begin{tabular}{|c|c|c|c|c|c|c|c|}
\hline $\begin{array}{c}\text { Peak } \\
\text { \# }\end{array}$ & $\begin{array}{l}\text { Time } \\
\text { [min] }\end{array}$ & $\begin{array}{l}\text { Area } \\
{[\mu \mathrm{V} \cdot \mathrm{s}]}\end{array}$ & $\begin{array}{l}\text { Height } \\
{[\mu \mathrm{V}]}\end{array}$ & $\begin{array}{c}\text { Area } \\
{[\%]}\end{array}$ & $\begin{array}{c}\text { Norm. Area } \\
{[\%]}\end{array}$ & $B L$ & $\begin{array}{c}\text { Area/Height } \\
{[\mathrm{s}]}\end{array}$ \\
\hline 1 & 1.527 & 765494.40 & 4282.17 & 6.70 & 6.70 & BB & 178.7633 \\
\hline 2 & 10.007 & 115320.80 & 4061.00 & 1.01 & 1.01 & BB & 28.3971 \\
\hline 3 & 10.947 & 18559.20 & 1325.00 & 0.16 & 0.16 & $\mathrm{BB}$ & 14.0069 \\
\hline 4 & 11.707 & 90122.44 & 5542.38 & 0.79 & 0.79 & $\mathrm{BB}$ & 16.2606 \\
\hline 5 & 12.607 & 10392161.96 & 373747.35 & 90.95 & 90.95 & MM & 27.8053 \\
\hline \multirow[t]{2}{*}{6} & 13.700 & 44735.00 & 2414.89 & 0.39 & 0.39 & $\mathrm{BB}$ & 18.5246 \\
\hline & & 11426393.80 & 391372.79 & 100.00 & 100.00 & & \\
\hline
\end{tabular}

Warning -- Signal level out-of-range in peak

Missing Component Report

Component Expected Retention (Calibration File)

All components were found 


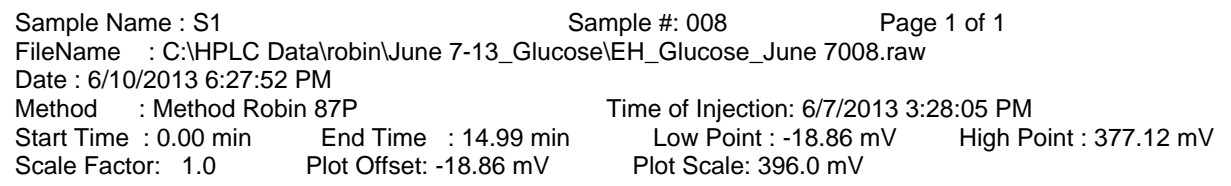

Response [mV]

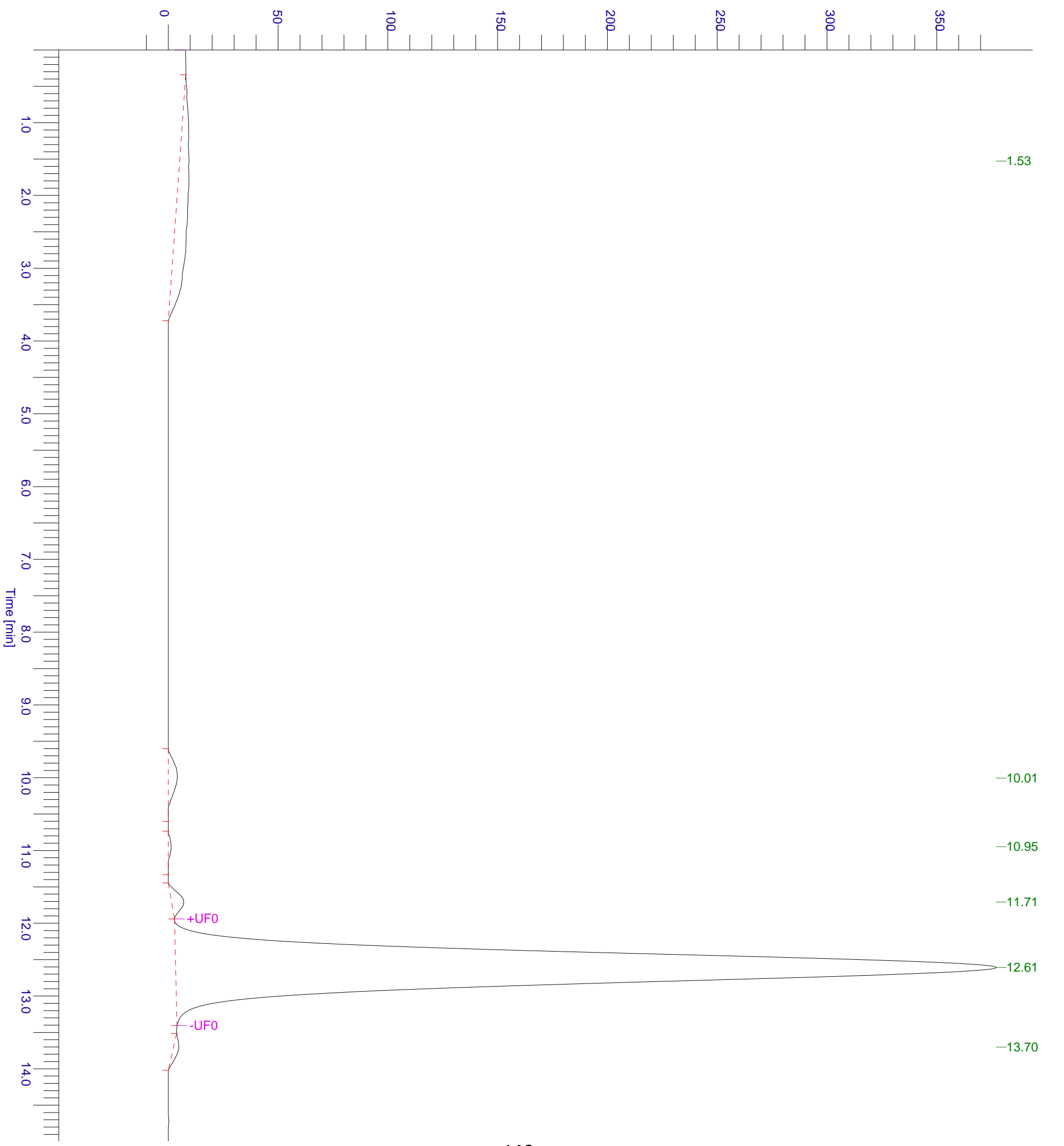




$\begin{array}{llll}\text { Software Version } & : 6.3 .2 .0646 & \text { Date } & : 6 / 11 / 201311: 16: 20 \text { AM } \\ \text { Operator } & : \text { rLuong } & \text { Sample Name } & : \text { S10 } \\ \text { Sample Number } & : \text { O17 } & \text { Study } & : \text { Glucose } \\ \text { AutoSampler } & : \text { SER200 } & \text { Rack/Vial } & : 1 / 17 \\ \text { Instrument Name } & : \text { HPLC } & \text { Channel } & : \text { A } \\ \text { Instrument Serial \# } & : \text { None } & \text { A/D mV Range }: 1000 \\ \text { Delay Time } & : 0.00 \mathrm{~min} & \text { End Time } & : 14.99 \mathrm{~min} \\ \text { Sampling Rate } & : 2.5000 \mathrm{pts} / \mathrm{s} & & \\ \text { Sample Volume } & : 1.000000 \mathrm{ul} & & \\ \text { Sample Amount } & : 1.0000 & \text { Area Reject } & : 0.000000 \\ \text { Data Acquisition Time } & : 6 / 7 / 20135: 52: 13 \mathrm{PM} & \text { Dilution Factor }: 1.00\end{array}$

Raw Data File : C:IHPLC Datalrobin June 7-13_GlucoselEH_Glucose_June 7017.raw

Result File : c:Ihplc datalrobinljune 7-13_glucoseleh_glucose_june 7017.rst [Editing in Progress]

Inst Method: C:IHPLC DatalrobinlMethod Robin 87P from C:IHPLC DatalrobinlJune 7-13 GlucoselEH Glucose June 7017.raw

Proc Method : C:IHPLC DatalrobinlMethod Robin 87P from c:Ihplc datalrobinljune 7-13_glucoseleh_glucose_june 7017.rst [Editing in Progress] Calib Method : C:IHPLC DatalrobinlMethod Robin 87P from c:Ihplc datalrobinljune 7-13_glucoseleh_glucose_june 7017.rst [Editing in Progress] Report Format File: C:IHPLC DatalrobinlMethod Robin 87P.rpt

Sequence File : C:IHPLC DatalrobinIValera Sequence 87p-glucose-June 7-13.seq

\begin{tabular}{|c|c|c|c|c|c|c|c|}
\hline $\begin{array}{c}\text { Peak } \\
\#\end{array}$ & $\begin{array}{l}\text { Time } \\
\text { [min] }\end{array}$ & $\begin{array}{c}\text { Area } \\
{[\mu \mathrm{V} \cdot \mathrm{s}]}\end{array}$ & $\begin{array}{l}\text { Height } \\
{[\mu \mathrm{V}]}\end{array}$ & $\begin{array}{c}\text { Area } \\
{[\%]}\end{array}$ & $\begin{array}{c}\text { Norm. Area } \\
{[\%]}\end{array}$ & $\mathrm{BL}$ & $\begin{array}{l}\text { Area/Height } \\
{[\mathrm{s}]}\end{array}$ \\
\hline 1 & 0.958 & 13808760.60 & 560482.86 & 60.13 & 60.13 & BB & 24.6373 \\
\hline 2 & 4.770 & 79620.60 & 912.36 & 0.35 & 0.35 & $\mathrm{BB}$ & 87.2688 \\
\hline 3 & 6.558 & 64882.33 & 2453.45 & 0.28 & 0.28 & BV & 26.4453 \\
\hline 4 & 7.093 & 165407.24 & 5974.69 & 0.72 & 0.72 & VV & 27.6847 \\
\hline 5 & 9.976 & 543415.43 & 7866.00 & 2.37 & 2.37 & VB & 69.0841 \\
\hline 6 & 11.673 & 127840.80 & 6725.96 & 0.56 & 0.56 & $\mathrm{BB}$ & 19.0071 \\
\hline 7 & 12.600 & 8111969.32 & 290151.09 & 35.32 & 35.32 & MM & 27.9577 \\
\hline 8 & 13.686 & 41986.00 & 1979.95 & 0.18 & 0.18 & $\mathrm{BB}$ & 21.2056 \\
\hline \multirow[t]{2}{*}{9} & 14.729 & 20678.80 & 1035.69 & 0.09 & 0.09 & BB & 19.9661 \\
\hline & & 22964561.12 & 877582.06 & L00.00 & 100.00 & & \\
\hline
\end{tabular}

Missing Component Report

Component Expected Retention (Calibration File)

All components were found 
FileName : C:IHPLC Datalrobin\June 7-13_GlucoselEH_Glucose_June 7017.raw

Date : 6/11/2013 11:16:46 AM

Method : Method Robin 87P

Start Time : $0.00 \mathrm{~min} \quad$ End Time : $14.99 \mathrm{~min}$

Time of Injection: 6/7/2013 5:52:13 PM

Scale Factor: $1.0 \quad$ Plot Offset: $-23.33 \mathrm{mV}$

Low Point : $-23.33 \mathrm{mV}$

High Point : $567.48 \mathrm{mV}$

Plot Scale: $590.8 \mathrm{mV}$

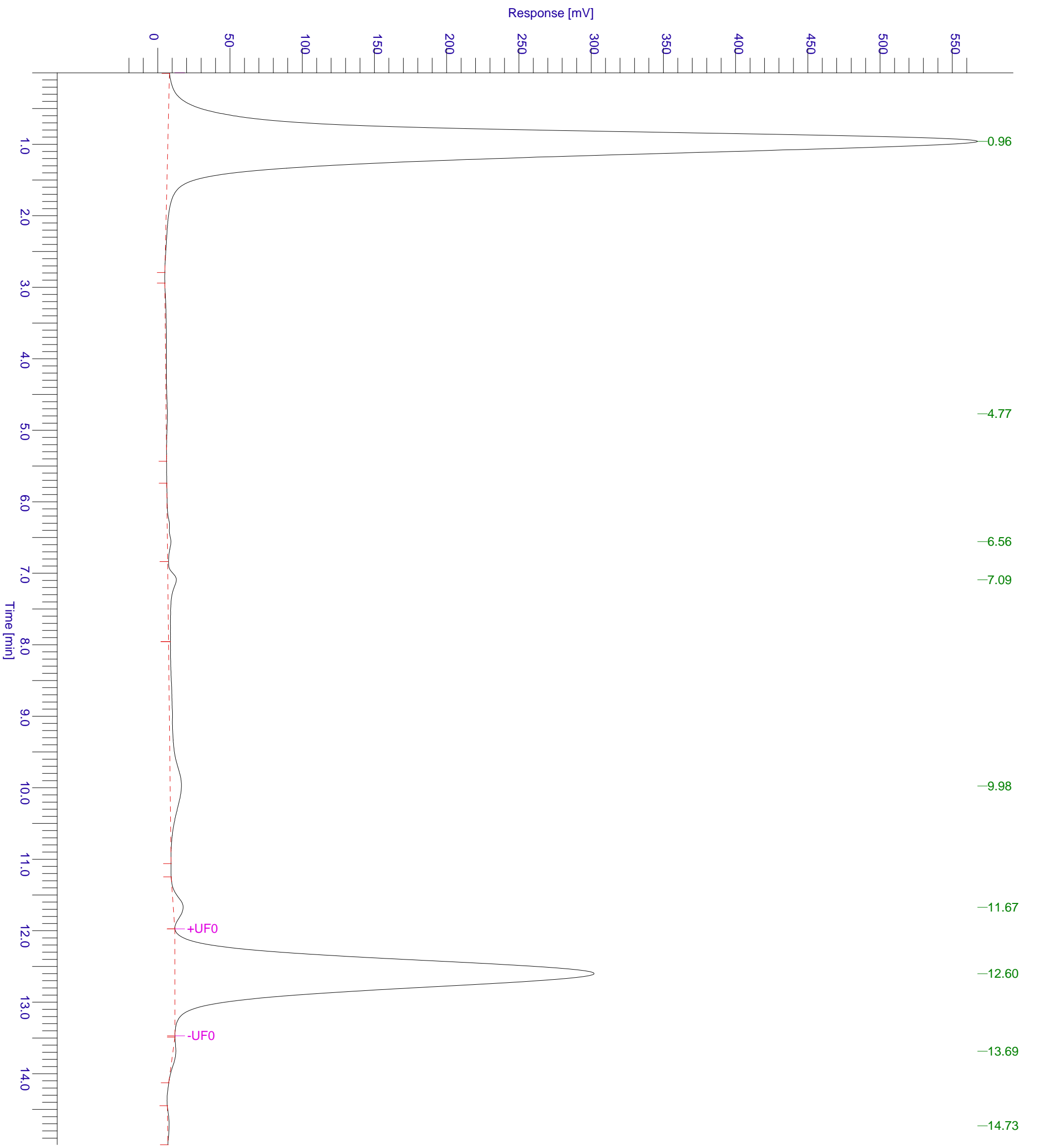




$\begin{array}{llll}\text { Software Version } & : 6.3 .2 .0646 & \text { Date } & : 6 / 10 / 2013 \text { 6:28:50 PM } \\ \text { Operator } & : \text { rLuong } & \text { Sample Name } & : \text { S2 } \\ \text { Sample Number } & : \text { O09 } & \text { Study } & : \text { Glucose } \\ \text { AutoSampler } & : \text { SER200 } & \text { Rack/Vial } & : 1 / 9 \\ \text { Instrument Name } & : \text { HPLC } & \text { Channel } & : \text { A } \\ \text { Instrument Serial \# } & : \text { None } & \text { A/D mV Range }: 1000 \\ \text { Delay Time } & : 0.00 \mathrm{~min} & \text { End Time } & : 14.99 \mathrm{~min} \\ \text { Sampling Rate } & : 2.5000 \mathrm{pts} / \mathrm{s} & & \\ \text { Sample Volume } & : 1.000000 \mathrm{ul} & & \\ \text { Sample Amount } & : 1.0000 & \text { Area Reject } & : 0.000000 \\ \text { Data Acquisition Time } & : 6 / 7 / 2013 \text { 3:44:06 PM } & \text { Dilution Factor }: 1.00\end{array}$

Raw Data File : C:IHPLC Datalrobin June 7-13_GlucoselEH_Glucose_June 7009.raw Result File : c:Ihplc datalrobinljune 7-13_glucoseleh_glucose_june 7009.rst [Editing in Progress] Inst Method: C:IHPLC DatalrobinlMethod Robin 87P from C:IHPLC DatalrobinlJune 7-13_GlucoselEH_Glucose_June 7009.raw Proc Method : C:IHPLC DatalrobinlMethod Robin 87P from c:Ihplc datalrobinljune 7-13_glucoseleh_glucose_june 7009.rst [Editing in Progress] Calib Method : C:IHPLC DatalrobinlMethod Robin 87P from c:Ihplc datalrobinljune 7-13_glucoseleh_glucose_june 7009.rst [Editing in Progress] Report Format File: C:IHPLC DatalrobinlMethod Robin 87P.rpt

Sequence File : C:IHPLC DatalrobinIValera Sequence 87p-glucose-June 7-13.seq

\begin{tabular}{|c|c|c|c|c|c|c|c|}
\hline $\begin{array}{c}\text { Peak } \\
\#\end{array}$ & $\begin{array}{l}\text { Time } \\
\text { [min] }\end{array}$ & $\begin{array}{c}\text { Area } \\
{[\mu \mathrm{V} \cdot \mathrm{s}]}\end{array}$ & $\begin{array}{c}\text { Height } \\
{[\mu \mathrm{V}]}\end{array}$ & $\begin{array}{c}\text { Area } \\
{[\%]}\end{array}$ & $\begin{array}{c}\text { Norm. Area } \\
{[\%]}\end{array}$ & $B L$ & $\begin{array}{c}\text { Area/Height } \\
\text { [s] }\end{array}$ \\
\hline 1 & 0.961 & 13768516.80 & 557905.49 & 55.59 & 55.59 & BB & 24.6789 \\
\hline 2 & 6.583 & 66255.20 & 2265.80 & 0.27 & 0.27 & BB & 29.2414 \\
\hline 3 & 7.093 & 118781.76 & 5859.92 & 0.48 & 0.48 & BV & 20.2702 \\
\hline 4 & 9.984 & 363543.40 & 7016.34 & 1.47 & 1.47 & VV & 51.8138 \\
\hline 5 & 10.962 & 76140.62 & 2944.20 & 0.31 & 0.31 & VB & 25.8613 \\
\hline 6 & 11.711 & 103361.60 & 5982.34 & 0.42 & 0.42 & $\mathrm{BB}$ & 17.2778 \\
\hline 7 & 12.613 & 10182019.12 & 366144.60 & 41.11 & 41.11 & MM & 27.8087 \\
\hline 8 & 13.689 & 51729.20 & 2498.78 & 0.21 & 0.21 & BB & 20.7018 \\
\hline \multirow[t]{2}{*}{9} & 14.721 & 39064.80 & 1858.74 & 0.16 & 0.16 & $\mathrm{BB}$ & 21.0168 \\
\hline & & 24769412.51 & 952476.21 & 00.00 & 100.00 & & \\
\hline
\end{tabular}

Missing Component Report

Component Expected Retention (Calibration File)

All components were found 

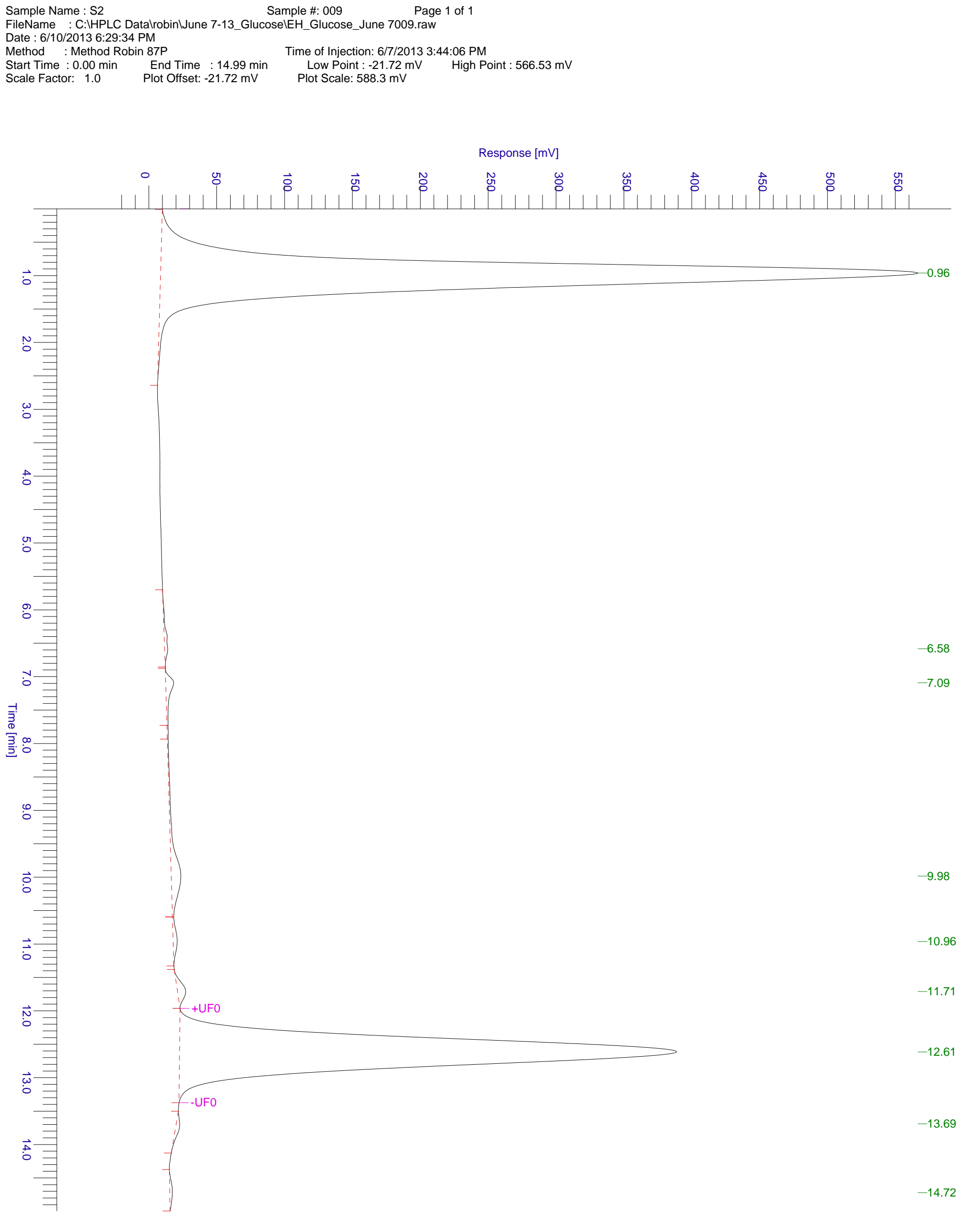


$\begin{array}{llll}\text { Software Version } & : 6.3 .2 .0646 & \text { Date } & : 6 / 10 / 20136: 31: 01 \mathrm{PM} \\ \text { Operator } & : \text { rLuong } & \text { Sample Name } & : \text { S3 } \\ \text { Sample Number } & : \text { O10 } & \text { Study } & : \text { Glucose } \\ \text { AutoSampler } & : \text { SER200 } & \text { Rack/Vial } & : 1 / 10 \\ \text { Instrument Name } & : \text { HPLC } & \text { Channel } & : \text { A } \\ \text { Instrument Serial \# } & : \text { None } & \text { A/D mV Range }: 1000 \\ \text { Delay Time } & : 0.00 \mathrm{~min} & \text { End Time } & : 14.99 \mathrm{~min} \\ \text { Sampling Rate } & : 2.5000 \mathrm{pts} / \mathrm{s} & & \\ \text { Sample Volume } & : 1.000000 \mathrm{ul} & & \\ \text { Sample Amount } & : 1.0000 & \text { Area Reject } & : 0.000000 \\ \text { Data Acquisition Time } & : 6 / 7 / 20134: 00: 10 \mathrm{PM} & \text { Dilution Factor }: 1.00\end{array}$

Raw Data File : C:IHPLC Datalrobin June 7-13_GlucoselEH_Glucose_June 7010.raw

Result File : c:Ihplc datalrobinljune 7-13_glucoseleh_glucose_june 7010.rst [Editing in Progress]

Inst Method: C:IHPLC DatalrobinlMethod Robin 87P from C:IHPLC DatalrobinlJune 7-13 GlucoselEH Glucose June 7010.raw

Proc Method : C:IHPLC DatalrobinlMethod Robin 87P from c:Ihplc datalrobinljune 7-13_glucoseleh_glucose_june 7010.rst [Editing in Progress] Calib Method : C:IHPLC DatalrobinlMethod Robin 87P from c:Ihplc datalrobinljune 7-13_glucoseleh_glucose_june 7010.rst [Editing in Progress] Report Format File: C:IHPLC DatalrobinlMethod Robin 87P.rpt

Sequence File : C:IHPLC DatalrobinIValera Sequence 87p-glucose-June 7-13.seq

\begin{tabular}{|c|c|c|c|c|c|c|c|}
\hline $\begin{array}{c}\text { Peak } \\
\#\end{array}$ & $\begin{array}{l}\text { Time } \\
\text { [min] }\end{array}$ & $\begin{array}{l}\text { Area } \\
{[\mu \mathrm{V} \cdot \mathrm{s}]}\end{array}$ & $\begin{array}{l}\text { Height } \\
{[\mu \mathrm{V}]}\end{array}$ & $\begin{array}{c}\text { Area } \\
{[\%]}\end{array}$ & $\begin{array}{c}\text { Norm. Area } \\
{[\%]}\end{array}$ & $B L$ & $\begin{array}{c}\text { Area/Height } \\
\text { [s] }\end{array}$ \\
\hline 1 & 0.902 & 13966610.40 & 568541.53 & 58.46 & 58.46 & $\mathrm{BB}$ & 24.5657 \\
\hline 2 & 3.623 & 68713.60 & 1110.13 & 0.29 & 0.29 & BB & 61.8969 \\
\hline 3 & 6.374 & 39718.40 & 1681.31 & 0.17 & 0.17 & BB & 23.6235 \\
\hline 4 & 7.093 & 98591.20 & 5313.00 & 0.41 & 0.41 & $\mathrm{BB}$ & 18.5566 \\
\hline 5 & 9.968 & 292326.64 & 6327.39 & 1.22 & 1.22 & BV & 46.2002 \\
\hline 6 & 10.982 & 27547.56 & 1284.56 & 0.12 & 0.12 & VB & 21.4452 \\
\hline 7 & 11.710 & 108306.80 & 6252.86 & 0.45 & 0.45 & BB & 17.3212 \\
\hline 8 & 12.613 & 9189055.08 & 331215.49 & 38.46 & 38.46 & MM & 27.7434 \\
\hline 9 & 13.692 & 62056.40 & 2741.10 & 0.26 & 0.26 & $\mathrm{BB}$ & 22.6392 \\
\hline \multirow[t]{2}{*}{10} & 14.715 & 37177.20 & 1858.03 & 0.16 & 0.16 & BB & 20.0089 \\
\hline & & 23890103.28 & 926325.40 & 100.00 & 100.00 & & \\
\hline
\end{tabular}

Missing Component Report

Component Expected Retention (Calibration File)

All components were found 
FileName : C:IHPLC DatalrobinlJune 7-13_GlucoselEH_Glucose_June 7010.raw

Date : 6/10/2013 6:31:32 PM

Method : Method Robin 87P

Start Time : $0.00 \mathrm{~min} \quad$ End Time : $14.99 \mathrm{~min}$

Time of Injection: 6/7/2013 4:00:10 PM

Scale Factor: $1.0 \quad$ Plot Offset: $-28.60 \mathrm{mV}$

Low Point : $-28.60 \mathrm{mV}$

High Point : $579.94 \mathrm{mV}$

Plot Scale: $608.5 \mathrm{mV}$

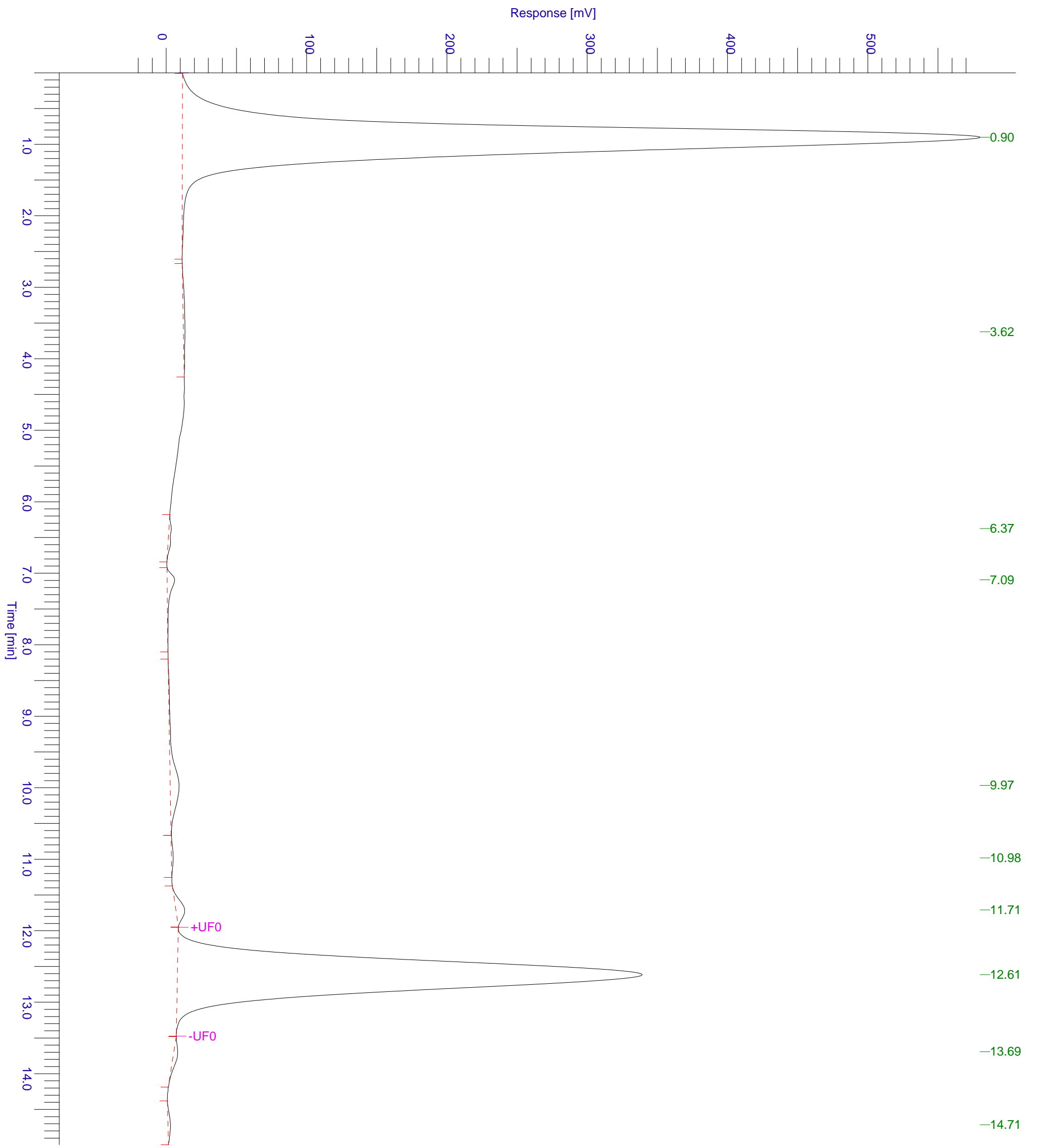




$\begin{array}{llll}\text { Software Version } & : 6.3 .2 .0646 & \text { Date } & : 6 / 11 / 2013 \text { 11:08:38 AM } \\ \text { Operator } & : \text { rLuong } & \text { Sample Name }: \text { S4 } \\ \text { Sample Number } & : \text { O11 } & \text { Study } & : \text { Glucose } \\ \text { AutoSampler } & : \text { SER200 } & \text { Rack/Vial } & : 1 / 11 \\ \text { Instrument Name } & : \text { HPLC } & \text { Channel } & : \text { A } \\ \text { Instrument Serial \# } & : \text { None } & \text { A/D mV Range }: 1000 \\ \text { Delay Time } & : 0.00 \mathrm{~min} & \text { End Time } & : 14.99 \mathrm{~min} \\ \text { Sampling Rate } & : 2.5000 \mathrm{pts} / \mathrm{s} & & \\ \text { Sample Volume } & : 1.000000 \mathrm{ul} & & \\ \text { Sample Amount } & : 1.0000 & \text { Area Reject } & : 0.000000 \\ \text { Data Acquisition Time } & : 6 / 7 / 20134: 16: 12 \mathrm{PM} & \text { Dilution Factor }: 1.00\end{array}$

Raw Data File : C:IHPLC Datalrobin June 7-13_GlucoselEH_Glucose_June 7011.raw

Result File : c:Ihplc datalrobinljune 7-13_glucoseleh_glucose_june 7011.rst [Editing in Progress]

Inst Method : C:IHPLC DatalrobinlMethod Robin 87P from C:IHPLC DatalrobinlJune 7-13 GlucoselEH Glucose June 7011.raw

Proc Method : C:IHPLC DatalrobinlMethod Robin 87P from c:Ihplc datalrobinljune 7-13_glucoseleh_glucose_june 7011.rst [Editing in Progress] Calib Method : C:IHPLC DatalrobinlMethod Robin 87P from c:Ihplc datalrobinljune 7-13_glucoseleh_glucose_june 7011.rst [Editing in Progress] Report Format File: C:IHPLC DatalrobinlMethod Robin 87P.rpt

Sequence File : C:IHPLC DatalrobinIValera Sequence 87p-glucose-June 7-13.seq

\begin{tabular}{|c|c|c|c|c|c|c|c|}
\hline $\begin{array}{c}\text { Peak } \\
\#\end{array}$ & $\begin{array}{l}\text { Time } \\
\text { [min] }\end{array}$ & $\begin{array}{l}\text { Area } \\
{[\mu \mathrm{V} \cdot \mathrm{s}]}\end{array}$ & $\begin{array}{l}\text { Height } \\
{[\mu \mathrm{V}]}\end{array}$ & $\begin{array}{c}\text { Area } \\
{[\%]}\end{array}$ & $\begin{array}{c}\text { Norm. Area } \\
{[\%]}\end{array}$ & $B L$ & $\begin{array}{c}\text { Area/Height } \\
\text { [s] }\end{array}$ \\
\hline 1 & 0.930 & 13931651.40 & 564347.27 & 61.27 & 61.27 & $\mathrm{BB}$ & 24.6863 \\
\hline 2 & 3.736 & 53043.18 & 783.74 & 0.23 & 0.23 & BV & 67.6799 \\
\hline 3 & 4.711 & 27901.22 & 774.65 & 0.12 & 0.12 & VB & 36.0177 \\
\hline 4 & 6.566 & 77047.60 & 2669.79 & 0.34 & 0.34 & BV & 28.8590 \\
\hline 5 & 7.090 & 160632.13 & 6304.13 & 0.71 & 0.71 & VV & 25.4805 \\
\hline 6 & 9.971 & 496745.28 & 7470.19 & 2.18 & 2.18 & VB & 66.4970 \\
\hline 7 & 11.711 & 124848.40 & 6825.90 & 0.55 & 0.55 & BB & 18.2904 \\
\hline 8 & 12.607 & 7765087.75 & 279818.61 & 34.15 & 34.15 & MM & 27.7504 \\
\hline 9 & 13.678 & 62746.40 & 2681.57 & 0.28 & 0.28 & BB & 23.3992 \\
\hline \multirow[t]{2}{*}{10} & 14.742 & 38408.00 & 1824.29 & 0.17 & 0.17 & BB & 21.0536 \\
\hline & & 22738111.35 & 873500.15 & 100.00 & 100.00 & & \\
\hline
\end{tabular}

Missing Component Report

Component Expected Retention (Calibration File)

All components were found 
FileName : C:IHPLC DatalrobinlJune 7-13_GlucoselEH_Glucose_June 7011.raw

Date : 6/11/2013 11:09:21 AM

Method : Method Robin 87P

Start Time : $0.00 \mathrm{~min} \quad$ End Time : $14.99 \mathrm{~min}$

Time of Injection: 6/7/2013 4:16:12 PM

Scale Factor: $1.0 \quad$ Plot Offset: $-23.88 \mathrm{mV}$

Low Point : $-23.88 \mathrm{mV}$

High Point : $572.31 \mathrm{mV}$

Plot Scale: $596.2 \mathrm{mV}$

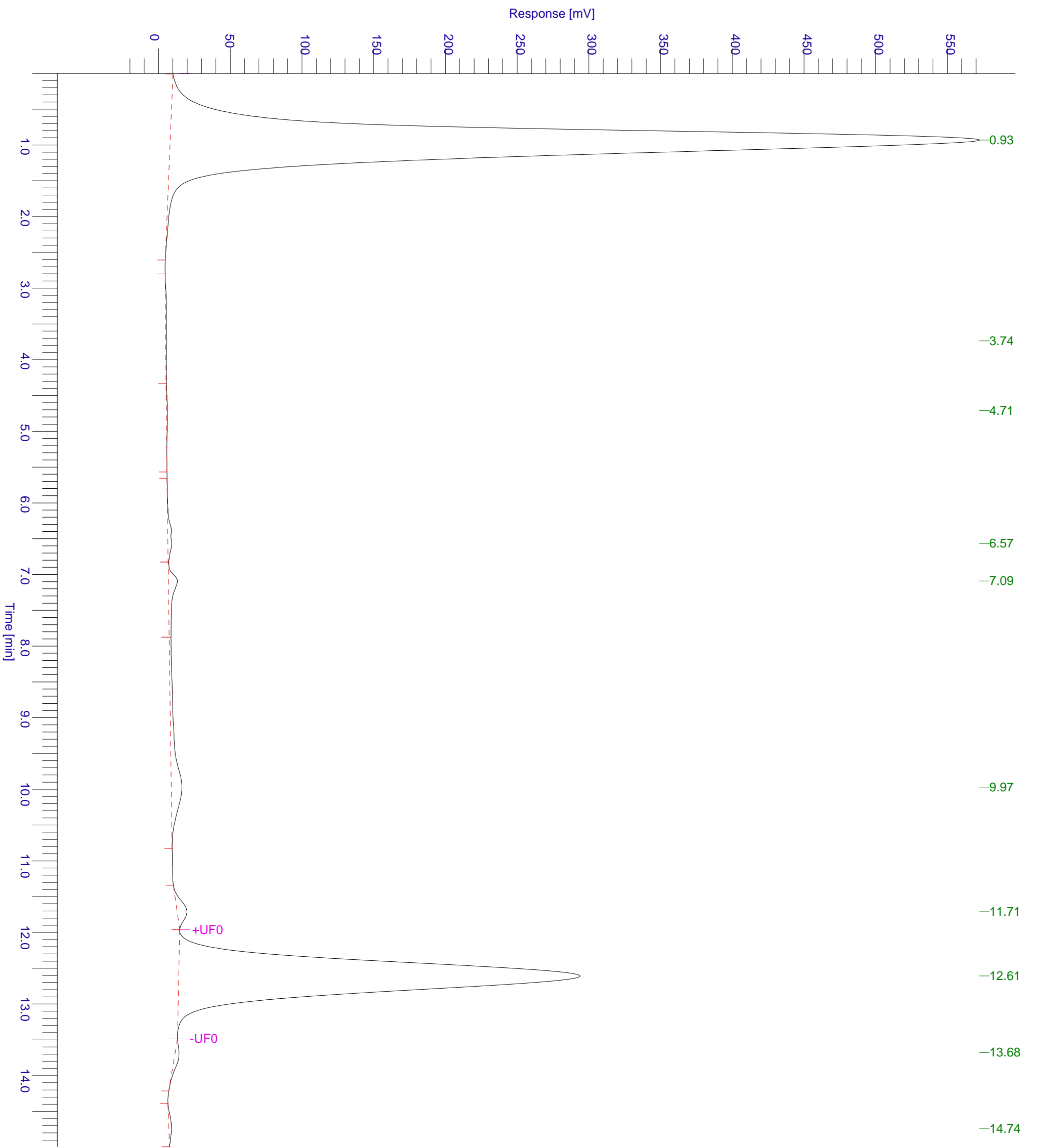




$\begin{array}{llll}\text { Software Version } & : 6.3 .2 .0646 & \text { Date } & : 6 / 11 / 2013 \text { 11:10:19 AM } \\ \text { Operator } & : \text { rLuong } & \text { Sample Name } & : \text { S5 } \\ \text { Sample Number } & : \text { O12 } & \text { Study } & : \text { Glucose } \\ \text { AutoSampler } & : \text { SER200 } & \text { Rack/Vial } & : 1 / 12 \\ \text { Instrument Name } & : \text { HPLC } & \text { Channel } & : \text { A } \\ \text { Instrument Serial \# } & : \text { None } & \text { A/D mV Range }: 1000 \\ \text { Delay Time } & : 0.00 \mathrm{~min} & \text { End Time } & : 14.99 \mathrm{~min} \\ \text { Sampling Rate } & : 2.5000 \mathrm{pts} / \mathrm{s} & & \\ \text { Sample Volume } & : 1.000000 \mathrm{ul} & & \\ \text { Sample Amount } & : 1.0000 & \text { Area Reject } & : 0.000000 \\ \text { Data Acquisition Time } & : 6 / 7 / 20134: 32: 12 \mathrm{PM} & \text { Dilution Factor }: 1.00\end{array}$

Raw Data File : C:IHPLC Datalrobin June 7-13_GlucoselEH_Glucose_June 7012.raw

Result File : c:Ihplc datalrobinljune 7-13_glucoseleh_glucose_june 7012.rst [Editing in Progress]

Inst Method: C:IHPLC DatalrobinlMethod Robin 87P from C:IHPLC DatalrobinlJune 7-13 GlucoselEH Glucose June 7012.raw

Proc Method : C:IHPLC DatalrobinlMethod Robin 87P from c:Ihplc datalrobinljune 7-13_glucoseleh_glucose_june 7012.rst [Editing in Progress] Calib Method : C:IHPLC DatalrobinlMethod Robin 87P from c:Ihplc datalrobinljune 7-13_glucoseleh_glucose_june 7012.rst [Editing in Progress] Report Format File: C:IHPLC DatalrobinlMethod Robin 87P.rpt

Sequence File : C:IHPLC DatalrobinIValera Sequence 87p-glucose-June 7-13.seq

\begin{tabular}{|c|c|c|c|c|c|c|c|}
\hline $\begin{array}{c}\text { Peak } \\
\quad \#\end{array}$ & $\begin{array}{l}\text { Time } \\
\text { [min] }\end{array}$ & $\begin{array}{l}\text { Area } \\
{[\mu \mathrm{V} \cdot \mathrm{s}]}\end{array}$ & $\begin{array}{l}\text { Height } \\
{[\mu \mathrm{V}]}\end{array}$ & $\begin{array}{c}\text { Area } \\
{[\%]}\end{array}$ & $\begin{array}{c}\text { Norm. Area } \\
{[\%]}\end{array}$ & BL & $\begin{array}{c}\text { Area/Height } \\
\text { [s] }\end{array}$ \\
\hline 1 & 0.964 & 14439082.00 & 583336.43 & 73.39 & 73.39 & BB & 24.7526 \\
\hline 2 & 3.773 & 125110.40 & 3431.42 & 0.64 & 0.64 & BB & 36.4603 \\
\hline 3 & 6.578 & 71493.32 & 2539.12 & 0.36 & 0.36 & BV & 28.1567 \\
\hline 4 & 7.101 & 135619.99 & 5888.98 & 0.69 & 0.69 & VV & 23.029 \\
\hline 5 & 9.992 & 449335.33 & 6719.11 & 2.28 & 2.28 & VB & 66.8742 \\
\hline 6 & 11.714 & 120743.60 & 6384.70 & 0.61 & 0.61 & $\mathrm{BB}$ & 18.911 \\
\hline 7 & 12.620 & 4223898.23 & 152384.21 & 21.47 & 21.47 & MM & 27.718 \\
\hline 8 & 13.715 & 73686.20 & 3259.28 & 0.37 & 0.37 & BB & 22.608 \\
\hline 9 & 14.760 & 35343.60 & 1661.33 & 0.18 & 0.18 & $\mathrm{BB}$ & 21.274 \\
\hline & & 19674312.67 & 5604.57 & 0.00 & 100.00 & & \\
\hline
\end{tabular}

Missing Component Report

Component Expected Retention (Calibration File)

All components were found 


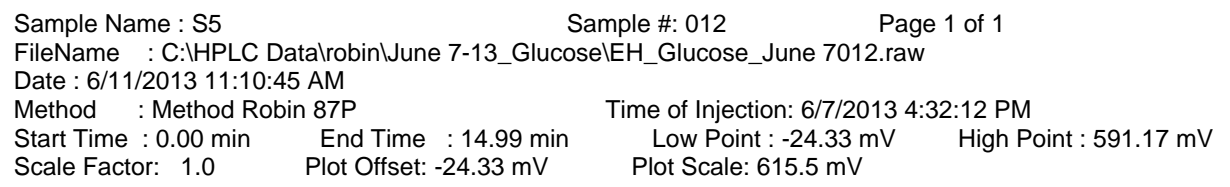

Response [mV]

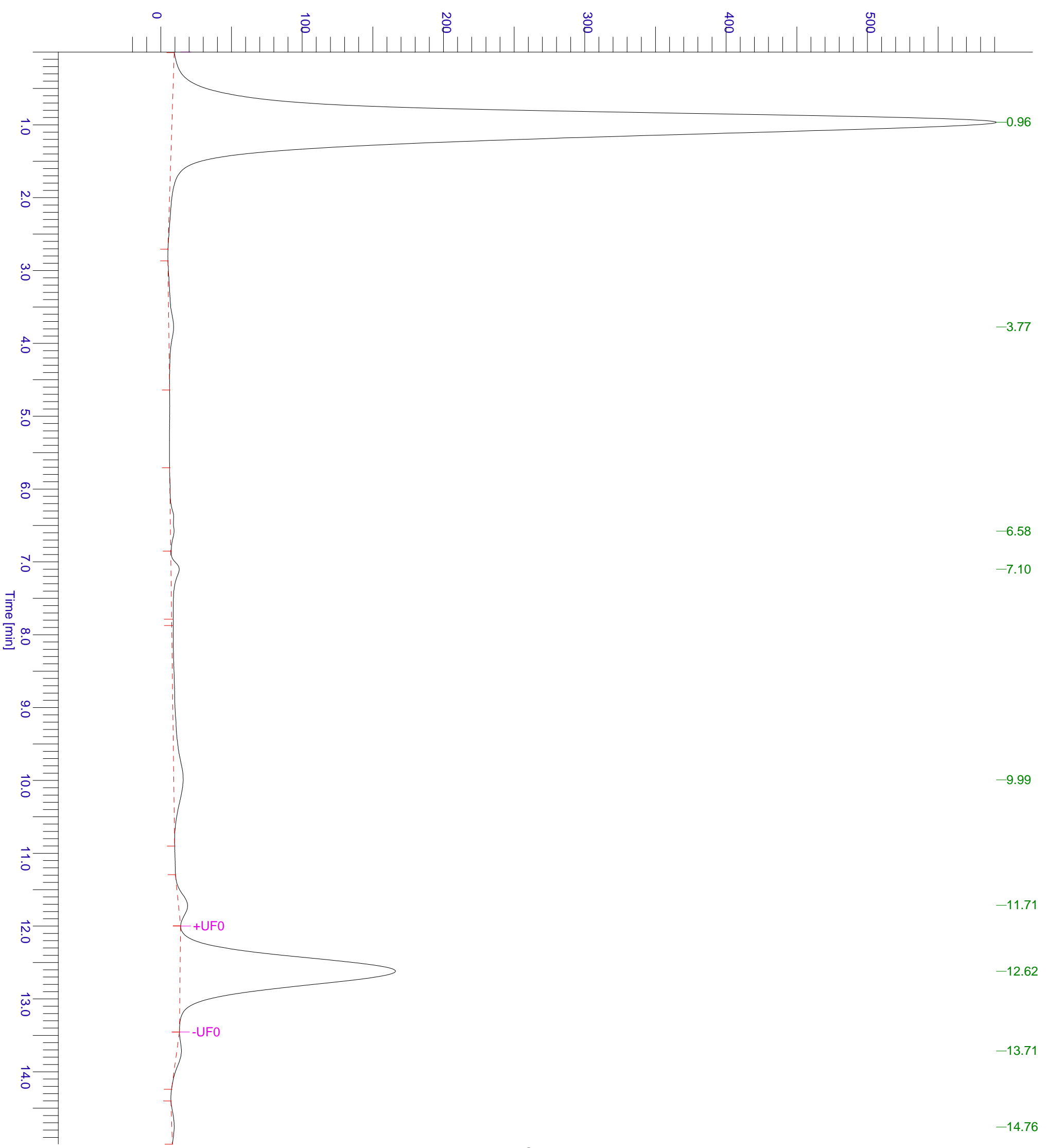




$\begin{array}{llll}\text { Software Version } & : 6.3 .2 .0646 & \text { Date } & : 6 / 11 / 201311: 11: 39 \text { AM } \\ \text { Operator } & : \text { rLuong } & \text { Sample Name } & : \text { S6 } \\ \text { Sample Number } & : 013 & \text { Study } & : \text { Glucose } \\ \text { AutoSampler } & : \text { SER200 } & \text { Rack/Vial } & : 1 / 13 \\ \text { Instrument Name } & : \text { HPLC } & \text { Channel } & : \text { A } \\ \text { Instrument Serial \# } & : \text { None } & \text { A/D mV Range }: 1000 \\ \text { Delay Time } & : 0.00 \mathrm{~min} & \text { End Time } & : 14.99 \mathrm{~min} \\ \text { Sampling Rate } & : 2.5000 \mathrm{pts} / \mathrm{s} & & \\ \text { Sample Volume } & : 1.000000 \mathrm{ul} & & \\ \text { Sample Amount } & : 1.0000 & \text { Area Reject } & : 0.000000 \\ \text { Data Acquisition Time } & : 6 / 7 / 2013 \mathrm{4:48:13} \mathrm{PM} & \text { Dilution Factor }: 1.00\end{array}$

Raw Data File : C:IHPLC Datalrobin June 7-13_GlucoselEH_Glucose_June 7013.raw

Result File : c:Ihplc datalrobinljune 7-13_glucoseleh_glucose_june 7013.rst [Editing in Progress]

Inst Method : C:IHPLC DatalrobinlMethod Robin 87P from C:IHPLC DatalrobinlJune 7-13 GlucoselEH Glucose June 7013.raw

Proc Method : C:IHPLC DatalrobinlMethod Robin 87P from c:Ihplc datalrobinljune 7-13_glucoseleh_glucose_june 7013.rst [Editing in Progress] Calib Method : C:IHPLC DatalrobinlMethod Robin 87P from c:Ihplc datalrobinljune 7-13_glucoseleh_glucose_june 7013.rst [Editing in Progress] Report Format File: C:IHPLC DatalrobinlMethod Robin 87P.rpt

Sequence File : C:IHPLC DatalrobinIValera Sequence 87p-glucose-June 7-13.seq

\begin{tabular}{|c|c|c|c|c|c|c|c|}
\hline $\begin{array}{c}\text { Peak } \\
\quad \#\end{array}$ & $\begin{array}{l}\text { Time } \\
\text { [min] }\end{array}$ & $\begin{array}{l}\text { Area } \\
{[\mu \mathrm{V} \cdot \mathrm{s}]}\end{array}$ & $\begin{array}{l}\text { Height } \\
{[\mu \mathrm{V}]}\end{array}$ & $\begin{array}{c}\text { Area } \\
{[\%]}\end{array}$ & $\begin{array}{c}\text { Norm. Area } \\
{[\%]}\end{array}$ & $\mathrm{BL}$ & $\begin{array}{l}\text { Area/Height } \\
{[\mathrm{s}]}\end{array}$ \\
\hline 1 & 0.965 & 14634117.20 & 591146.50 & 84.99 & 84.99 & BB & 24.7555 \\
\hline 2 & 3.783 & 350603.60 & 11652.28 & 2.04 & 2.04 & BB & 30.0888 \\
\hline 3 & 6.564 & 75987.44 & 2654.65 & 0.44 & 0.44 & $\mathrm{BV}$ & 28.6242 \\
\hline 4 & 7.103 & 141886.73 & 5800.29 & 0.82 & 0.82 & VV & 24.4620 \\
\hline 5 & 9.999 & 436990.48 & 6541.62 & 2.54 & 2.54 & VB & 66.8016 \\
\hline 6 & 11.719 & 81642.80 & 4185.48 & 0.47 & 0.47 & $\mathrm{BB}$ & 19.5062 \\
\hline 7 & 12.613 & 1357805.17 & 49899.34 & 7.89 & 7.89 & MM & 27.2109 \\
\hline 8 & 13.713 & 100710.80 & 3828.30 & 0.58 & 0.58 & BB & 26.3069 \\
\hline \multirow[t]{2}{*}{9} & 14.754 & 38837.20 & 1873.16 & 0.23 & 0.23 & BB & 20.7335 \\
\hline & & 17218581.42 & 77581.62 & 00.00 & 100.00 & & \\
\hline
\end{tabular}

Missing Component Report

Component Expected Retention (Calibration File)

All components were found 

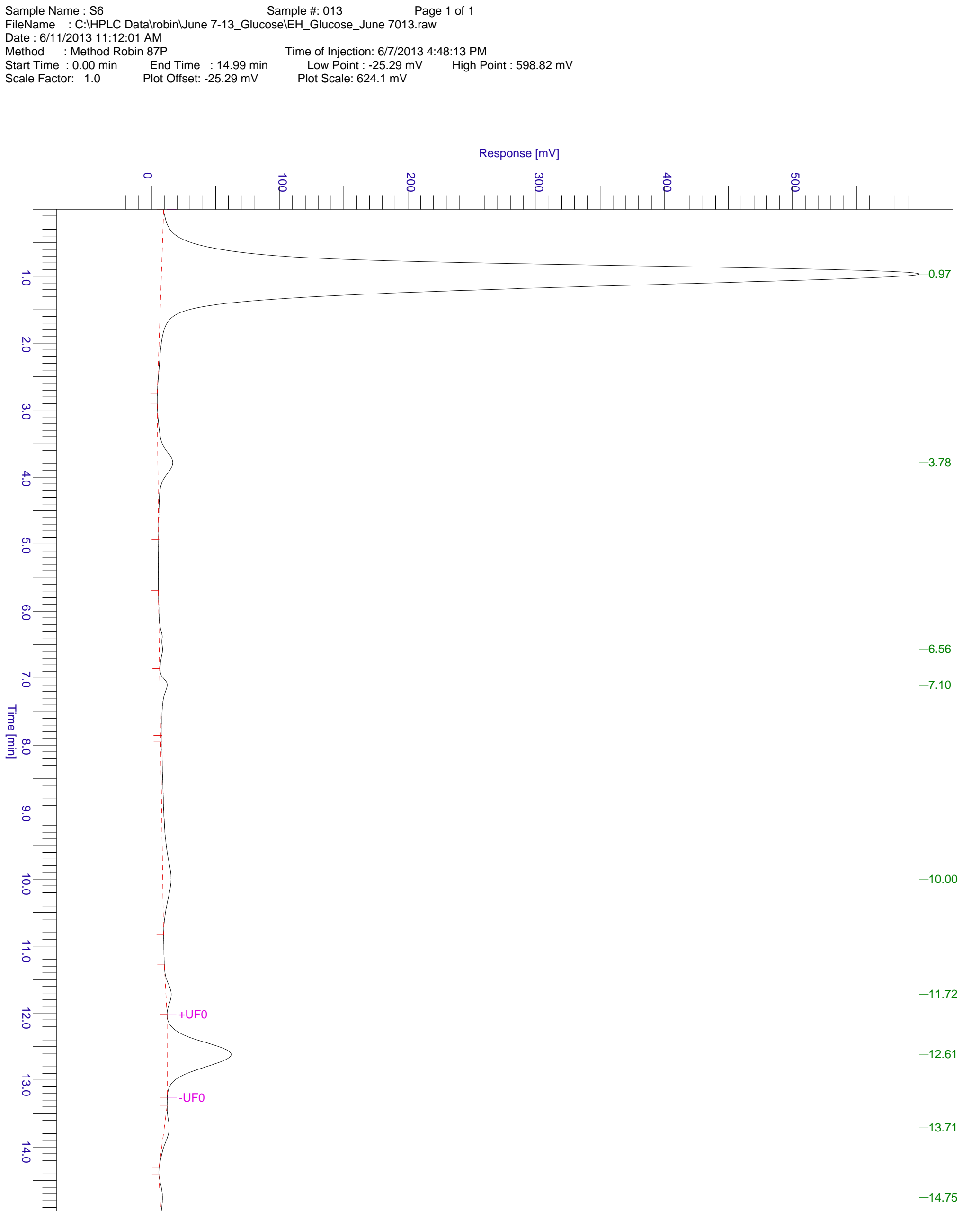


$\begin{array}{llll}\text { Software Version } & : 6.3 .2 .0646 & \text { Date } & : 6 / 11 / 201311: 13: 20 \text { AM } \\ \text { Operator } & : \text { rLuong } & \text { Sample Name } & : \text { S7 } \\ \text { Sample Number } & : \text { O14 } & \text { Study } & : \text { Glucose } \\ \text { AutoSampler } & : \text { SER200 } & \text { Rack/Vial } & : 1 / 14 \\ \text { Instrument Name } & : \text { HPLC } & \text { Channel } & : \text { A } \\ \text { Instrument Serial \# } & : \text { None } & \text { A/D mV Range }: 1000 \\ \text { Delay Time } & : 0.00 \mathrm{~min} & \text { End Time } & : 14.99 \mathrm{~min} \\ \text { Sampling Rate } & : 2.5000 \mathrm{pts} / \mathrm{s} & & \\ \text { Sample Volume } & : 1.000000 \mathrm{ul} & & \\ \text { Sample Amount } & : 1.0000 & \text { Area Reject } & : 0.000000 \\ \text { Data Acquisition Time } & : 6 / 7 / 2013 \mathrm{5:04:13} \mathrm{PM} & \text { Dilution Factor }: 1.00\end{array}$

Raw Data File : C:IHPLC Datalrobin June 7-13_GlucoselEH_Glucose_June 7014.raw

Result File : c:Ihplc datalrobinljune 7-13_glucoseleh_glucose_june 7014.rst [Editing in Progress]

Inst Method : C:IHPLC DatalrobinlMethod Robin 87P from C:IHPLC DatalrobinlJune 7-13 GlucoselEH Glucose June 7014.raw

Proc Method : C:IHPLC DatalrobinlMethod Robin 87P from c:Ihplc datalrobinljune 7-13_glucoseleh_glucose_june 7014.rst [Editing in Progress] Calib Method : C:IHPLC DatalrobinlMethod Robin 87P from c:Ihplc datalrobinljune 7-13_glucoseleh_glucose_june 7014.rst [Editing in Progress] Report Format File: C:IHPLC DatalrobinlMethod Robin 87P.rpt

Sequence File : C:IHPLC DatalrobinIValera Sequence 87p-glucose-June 7-13.seq

\begin{tabular}{|c|c|c|c|c|c|c|c|}
\hline $\begin{array}{c}\text { Peak } \\
\#\end{array}$ & $\begin{array}{l}\text { Time } \\
\text { [min] }\end{array}$ & $\begin{array}{c}\text { Area } \\
{[\mu \mathrm{V} \cdot \mathrm{s}]}\end{array}$ & $\begin{array}{c}\text { Height } \\
{[\mu \mathrm{V}]}\end{array}$ & $\begin{array}{c}\text { Area } \\
\text { [\%] }\end{array}$ & $\begin{array}{c}\text { Norm. Area } \\
{[\%]}\end{array}$ & $\mathrm{BL}$ & $\begin{array}{l}\text { Area/Height } \\
{[\mathrm{s}]}\end{array}$ \\
\hline 1 & 0.968 & 14884357.40 & 601287.55 & 57.54 & 57.54 & BB & 24.7541 \\
\hline 2 & 3.789 & 529471.60 & 16556.23 & 2.05 & 2.05 & BB & 31.9802 \\
\hline 3 & 6.580 & 61092.18 & 2194.09 & 0.24 & 0.24 & BV & 27.8440 \\
\hline 4 & 7.091 & 150174.56 & 5379.82 & 0.58 & 0.58 & VV & 27.9144 \\
\hline 5 & 9.960 & 509931.00 & 7471.14 & 1.97 & 1.97 & VV & 68.2535 \\
\hline 6 & 10.925 & 46912.02 & 2031.27 & 0.18 & 0.18 & VB & 23.0950 \\
\hline 7 & 11.709 & 90303.00 & 5184.21 & 0.35 & 0.35 & BB & 17.4189 \\
\hline \multirow[t]{2}{*}{8} & 12.607 & 9593459.50 & 342631.55 & 37.09 & 37.09 & MM & 27.9993 \\
\hline & & 25865701.27 & 982735.86 & 100.00 & 100.00 & & \\
\hline
\end{tabular}

Missing Component Report

Component Expected Retention (Calibration File)

All components were found 

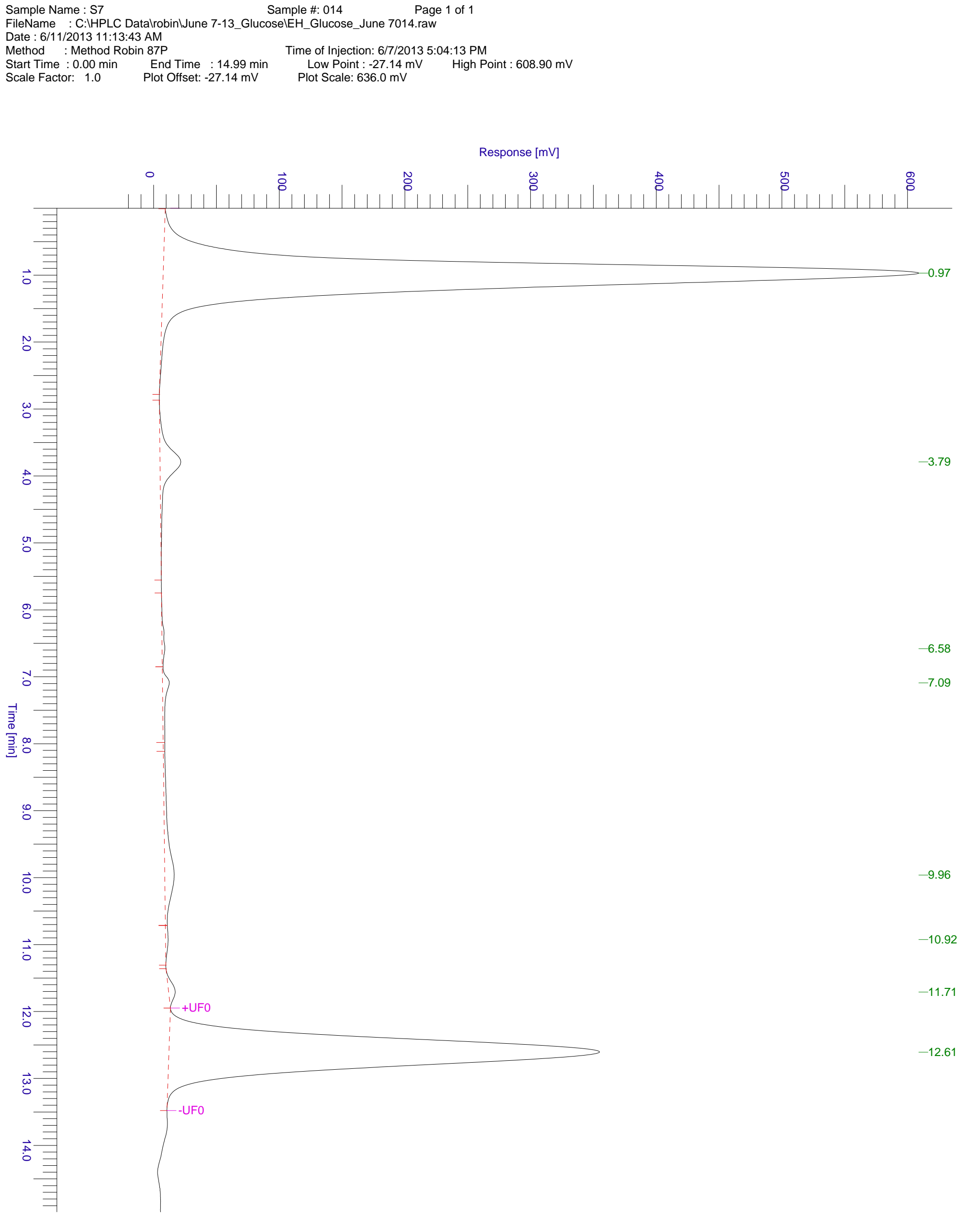


$\begin{array}{llll}\text { Software Version } & : 6.3 .2 .0646 & \text { Date } & : 6 / 11 / 201311: 14: 24 \text { AM } \\ \text { Operator } & : \text { rLuong } & \text { Sample Name } & : \text { S8 } \\ \text { Sample Number } & : 015 & \text { Study } & : \text { Glucose } \\ \text { AutoSampler } & : \text { SER200 } & \text { Rack/Vial } & : 1 / 15 \\ \text { Instrument Name } & : \text { HPLC } & \text { Channel } & : \text { A } \\ \text { Instrument Serial \# } & : \text { None } & \text { A/D mV Range }: 1000 \\ \text { Delay Time } & : 0.00 \mathrm{~min} & \text { End Time } & : 14.99 \mathrm{~min} \\ \text { Sampling Rate } & : 2.5000 \mathrm{pts} / \mathrm{s} & & \\ \text { Sample Volume } & : 1.000000 \mathrm{ul} & & \\ \text { Sample Amount } & : 1.0000 & \text { Area Reject } & : 0.000000 \\ \text { Data Acquisition Time } & : 6 / 7 / 20135: 20: 13 \mathrm{PM} & \text { Dilution Factor }: 1.00\end{array}$

Raw Data File : C:IHPLC Datalrobin June 7-13_GlucoselEH_Glucose_June 7015.raw

Result File : c:Ihplc datalrobinljune 7-13_glucoseleh_glucose_june 7015.rst [Editing in Progress]

Inst Method: C:IHPLC DatalrobinlMethod Robin 87P from C:IHPLC DatalrobinlJune 7-13 GlucoselEH Glucose June 7015.raw

Proc Method : C:IHPLC DatalrobinlMethod Robin 87P from c:Ihplc datalrobinljune 7-13_glucoseleh_glucose_june 7015.rst [Editing in Progress] Calib Method : C:IHPLC DatalrobinlMethod Robin 87P from c:Ihplc datalrobinljune 7-13_glucoseleh_glucose_june 7015.rst [Editing in Progress] Report Format File: C:IHPLC DatalrobinlMethod Robin 87P.rpt

Sequence File : C:IHPLC DatalrobinIValera Sequence 87p-glucose-June 7-13.seq

\begin{tabular}{|c|c|c|c|c|c|c|c|}
\hline $\begin{array}{c}\text { Peak } \\
\#\end{array}$ & $\begin{array}{l}\text { Time } \\
\text { [min] }\end{array}$ & $\begin{array}{c}\text { Area } \\
{[\mu \mathrm{V} \cdot \mathrm{s}]}\end{array}$ & $\begin{array}{c}\text { Height } \\
{[\mu \mathrm{V}]}\end{array}$ & $\begin{array}{c}\text { Area } \\
{[\%]}\end{array}$ & $\begin{array}{c}\text { Norm. Area } \\
{[\%]}\end{array}$ & $\mathrm{BL}$ & $\begin{array}{l}\text { Area/Height } \\
{[\mathrm{s}]}\end{array}$ \\
\hline 1 & 0.964 & 12217116.00 & 500394.26 & 53.52 & 53.52 & BB & 24.4150 \\
\hline 2 & 3.756 & 23028.00 & 454.29 & 0.10 & 0.10 & BB & 50.6903 \\
\hline 3 & 6.559 & 51681.99 & 2042.10 & 0.23 & 0.23 & BV & 25.3083 \\
\hline 4 & 7.105 & 125563.14 & 5324.94 & 0.55 & 0.55 & VV & 23.5802 \\
\hline 5 & 9.983 & 419991.56 & 6876.46 & 1.84 & 1.84 & VV & 61.0767 \\
\hline 6 & 10.927 & 58621.32 & 2161.37 & 0.26 & 0.26 & VB & 27.1224 \\
\hline 7 & 11.706 & 79564.80 & 5023.85 & 0.35 & 0.35 & BB & 15.8374 \\
\hline \multirow[t]{2}{*}{8} & 12.607 & 9853687.31 & 349773.56 & 43.16 & 43.16 & MM & 28.1716 \\
\hline & & 22829254.11 & 872050.83 & 100.00 & 100.00 & & \\
\hline
\end{tabular}

Missing Component Report

Component Expected Retention (Calibration File)

All components were found 


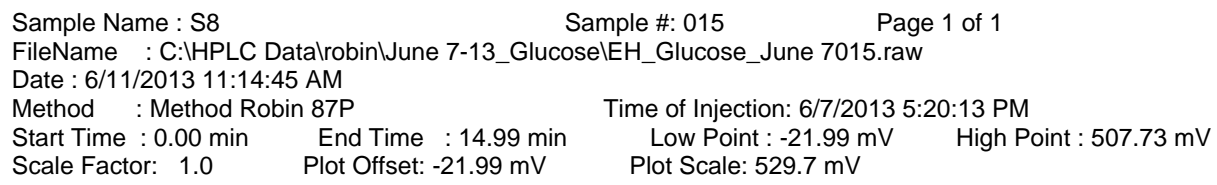

Response [mV]

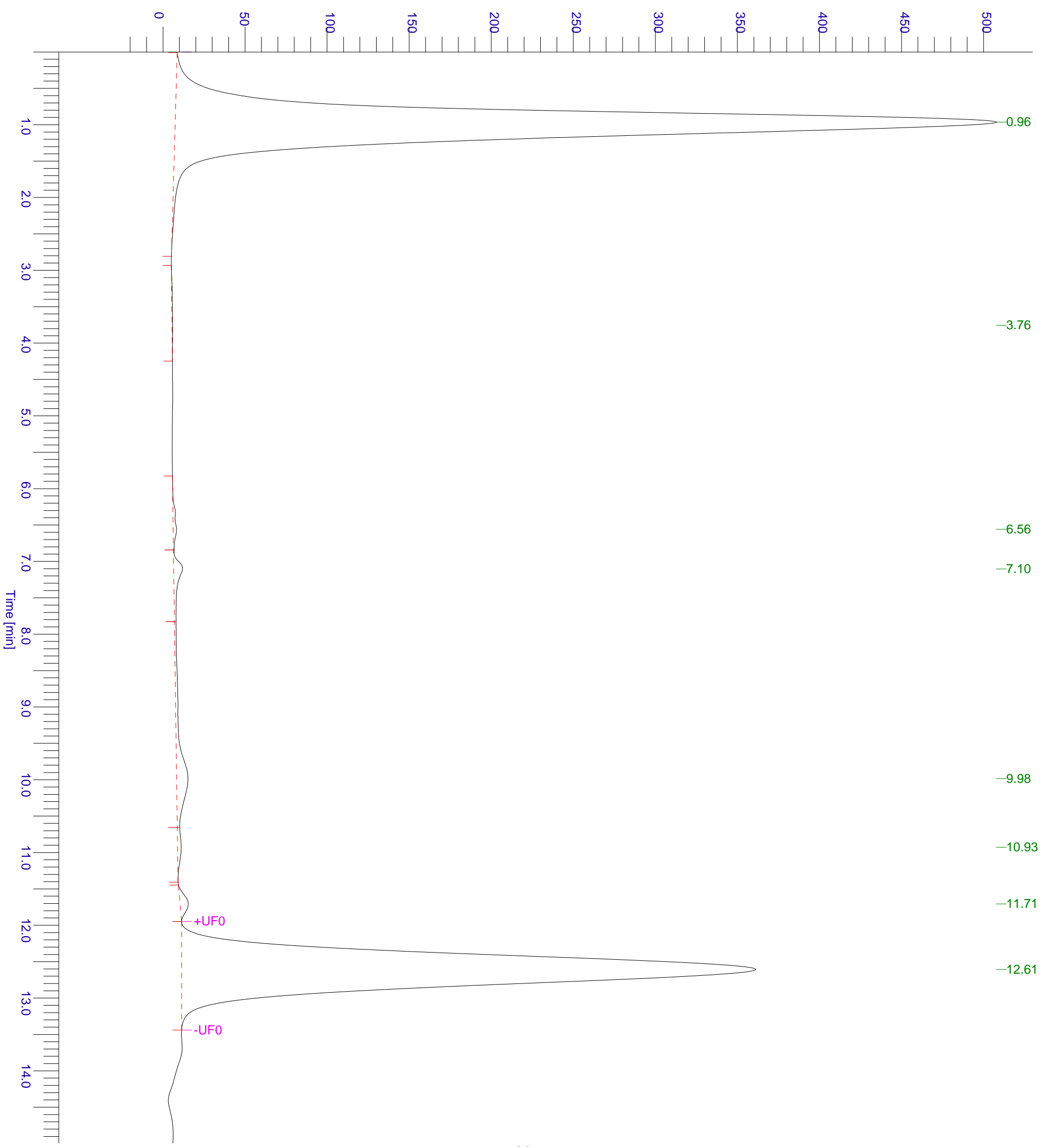




$\begin{array}{llll}\text { Software Version } & : 6.3 .2 .0646 & \text { Date } & : 6 / 11 / 201311: 15: 23 \text { AM } \\ \text { Operator } & : \text { rLuong } & \text { Sample Name } & : \text { S9 } \\ \text { Sample Number } & : 016 & \text { Study } & : \text { Glucose } \\ \text { AutoSampler } & : \text { SER200 } & \text { Rack/Vial } & : 1 / 16 \\ \text { Instrument Name } & : \text { HPLC } & \text { Channel } & : \text { A } \\ \text { Instrument Serial \# } & : \text { None } & \text { A/D mV Range }: 1000 \\ \text { Delay Time } & : 0.00 \mathrm{~min} & \text { End Time } & : 14.99 \mathrm{~min} \\ \text { Sampling Rate } & : 2.5000 \mathrm{pts} / \mathrm{s} & & \\ \text { Sample Volume } & : 1.000000 \mathrm{ul} & & \\ \text { Sample Amount } & : 1.0000 & \text { Area Reject } & : 0.000000 \\ \text { Data Acquisition Time } & : 6 / 7 / 20135: 36: 13 \mathrm{PM} & \text { Dilution Factor }: 1.00\end{array}$

Raw Data File : C:IHPLC Datalrobin June 7-13_GlucoselEH_Glucose_June 7016.raw

Result File : c:Ihplc datalrobinljune 7-13_glucoseleh_glucose_june 7016.rst [Editing in Progress]

Inst Method: C:IHPLC DatalrobinlMethod Robin 87P from C:IHPLC DatalrobinlJune 7-13 GlucoselEH Glucose June 7016.raw

Proc Method : C:IHPLC DatalrobinlMethod Robin 87P from c:Ihplc datalrobinljune 7-13_glucoseleh_glucose_june 7016.rst [Editing in Progress] Calib Method : C:IHPLC DatalrobinlMethod Robin 87P from c:Ihplc datalrobinljune 7-13_glucoseleh_glucose_june 7016.rst [Editing in Progress] Report Format File: C:IHPLC DatalrobinlMethod Robin 87P.rpt

Sequence File : C:IHPLC DatalrobinIValera Sequence 87p-glucose-June 7-13.seq

\begin{tabular}{|c|c|c|c|c|c|c|c|}
\hline $\begin{array}{c}\text { Peak } \\
\#\end{array}$ & $\begin{array}{l}\text { Time } \\
\text { [min] }\end{array}$ & $\begin{array}{c}\text { Area } \\
{[\mu \mathrm{V} \cdot \mathrm{s}]}\end{array}$ & $\begin{array}{c}\text { Height } \\
{[\mu \mathrm{V}]}\end{array}$ & $\begin{array}{c}\text { Area } \\
{[\%]}\end{array}$ & $\begin{array}{c}\text { Norm. Area } \\
{[\%]}\end{array}$ & $\mathrm{BL}$ & $\begin{array}{l}\text { Area/Height } \\
{[\mathrm{s}]}\end{array}$ \\
\hline 1 & 0.960 & 13189985.80 & 536040.70 & 55.74 & 55.74 & BB & 24.6063 \\
\hline 2 & 4.798 & 81742.00 & 976.84 & 0.35 & 0.35 & BB & 83.6802 \\
\hline 3 & 6.565 & 51286.97 & 2143.79 & 0.22 & 0.22 & BV & 23.9235 \\
\hline 4 & 7.093 & 156863.30 & 5625.16 & 0.66 & 0.66 & VV & 27.8860 \\
\hline 5 & 9.981 & 476257.73 & 7375.34 & 2.01 & 2.01 & VB & 64.5744 \\
\hline 6 & 11.701 & 103874.40 & 5644.53 & 0.44 & 0.44 & BB & 18.4027 \\
\hline 7 & 12.607 & 9579356.30 & 340995.65 & 40.48 & 40.48 & MM & 28.0923 \\
\hline \multirow[t]{2}{*}{8} & 14.750 & 26009.09 & 1313.68 & 0.11 & 0.11 & BB & 19.7987 \\
\hline & & 23665375.58 & 900115.67 & 100.00 & 100.00 & & \\
\hline
\end{tabular}

Missing Component Report

Component Expected Retention (Calibration File)

All components were found 

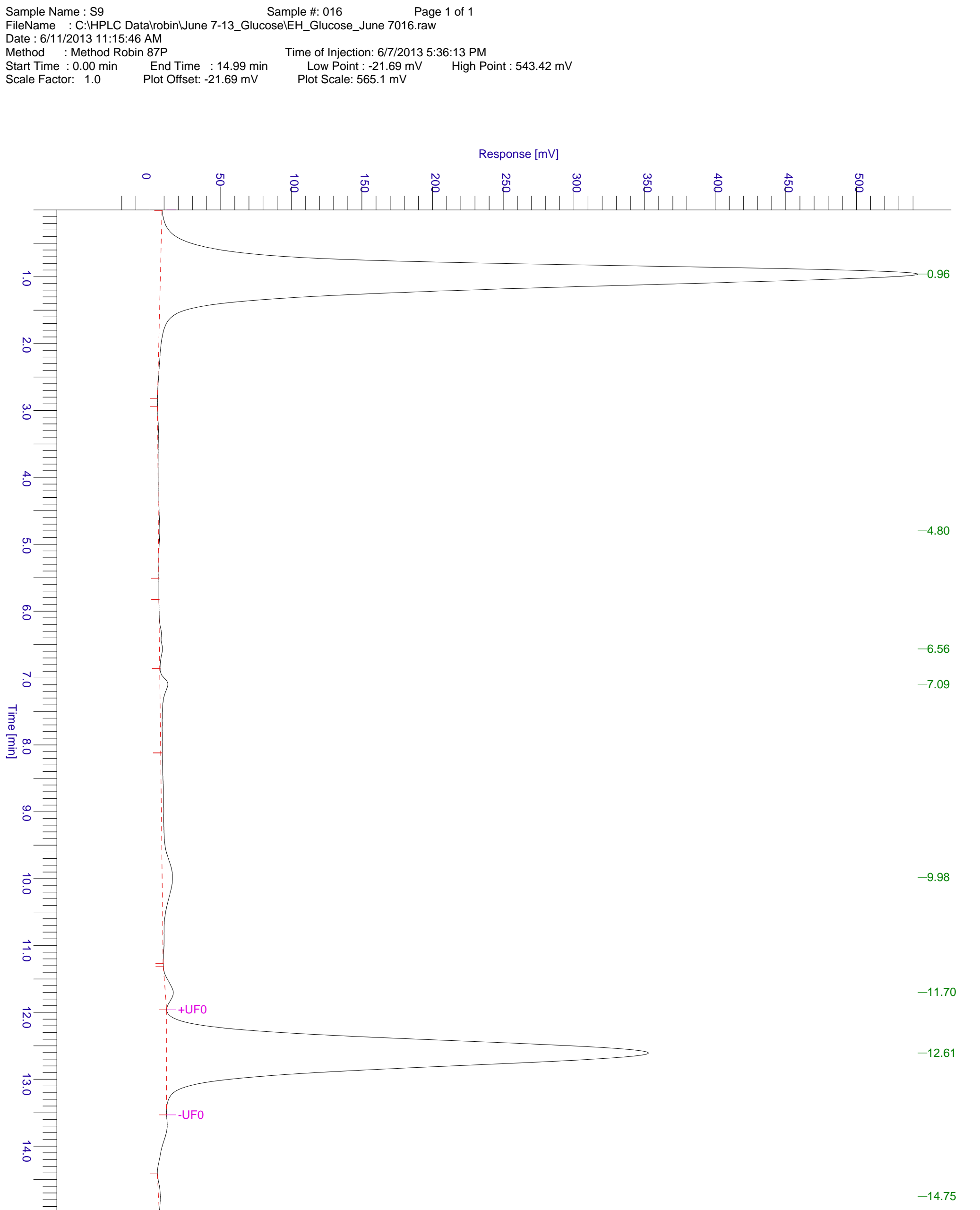


$\begin{array}{llll}\text { Software Version } & : 6.3 .2 .0646 & \text { Date } & : 6 / 21 / 2013 \text { 12:48:01 PM } \\ \text { Operator } & : \text { rLuong } & \text { Sample Name } & : \text { S1 } \\ \text { Sample Number } & : \text { O08 } & \text { Study } & : \text { Ethanol } \\ \text { AutoSampler } & : \text { SER200 } & \text { Rack/Vial } & : 1 / 8 \\ \text { Instrument Name } & : \text { HPLC } & \text { Channel } & : \text { A } \\ \text { Instrument Serial \# } & : \text { None } & \text { A/D mV Range }: 1000 \\ \text { Delay Time } & : 0.00 \mathrm{~min} & \text { End Time } & : 24.99 \mathrm{~min} \\ \text { Sampling Rate } & : 2.5000 \mathrm{pts} / \mathrm{s} & & \\ \text { Sample Volume } & : 1.000000 \mathrm{ul} & & \\ \text { Sample Amount } & : 1.0000 & \text { Area Reject } & : 0.000000 \\ \text { Data Acquisition Time } & : 6 / 11 / 2013 \text { 4:23:49 PM } & \text { Dilution Factor }: 1.00\end{array}$

Raw Data File : C:IHPLC DatalrobinlEthanol_June 10-2013lEthanol008.raw

Result File : c:Ihplc datalrobinlethanol_june 10-2013lethanol008.rst [Editing in Progress]

Inst Method : C:IHPLC DatalrobinlMethod Robin 87H from C:IHPLC DatalrobinlEthanol_June 10-2013।Ethanol008.raw

Proc Method : C:IHPLC DatalrobinlMethod Robin 87H from c:Ihplc datalrobinlethanol_june 10-2013lethanol008.rst [Editing in Progress] Calib Method : C:IHPLC DatalrobinlMethod Robin 87H from c:Ihplc datalrobinlethanol_june 10-2013lethanol008.rst [Editing in Progress]

Report Format File: C:IHPLC DatalrobinlMethod Robin 87H.rpt

Sequence File : C:IHPLC DatalrobinIValera Sequence 87H-Etanol_June 10_2013.seq

\begin{tabular}{|c|c|c|c|c|c|c|c|}
\hline $\begin{array}{c}\text { Peak } \\
\#\end{array}$ & $\begin{array}{l}\text { Time } \\
\text { [min] }\end{array}$ & $\begin{array}{l}\text { Area } \\
{[\mu \mathrm{V} \cdot \mathrm{s}]}\end{array}$ & $\begin{array}{l}\text { Height } \\
{[\mu \mathrm{V}]}\end{array}$ & $\begin{array}{c}\text { Area } \\
{[\%]}\end{array}$ & $\begin{array}{c}\text { Norm. Area } \\
{[\%]}\end{array}$ & $B L$ & $\begin{array}{c}\text { Area/Height } \\
{[\mathrm{s}]}\end{array}$ \\
\hline - & 0.001 & 0.00 & 0.00 & 0.00 & 0.00 & & \\
\hline 1 & 0.227 & 354.80 & 45.51 & 0.00 & 0.00 & BB & 7.7957 \\
\hline 2 & 0.340 & 275.00 & 59.88 & 0.00 & 0.00 & BB & 4.5923 \\
\hline 3 & 0.802 & 146.00 & 66.24 & 0.00 & 0.00 & BB & 2.2042 \\
\hline 4 & 0.976 & 76.20 & 51.69 & $7 e-05$ & $7 e-05$ & BB & 1.4740 \\
\hline 5 & 1.174 & 306.80 & 56.56 & 0.00 & 0.00 & BB & 5.4243 \\
\hline 6 & 1.452 & 313.20 & 65.50 & 0.00 & 0.00 & BB & 4.7818 \\
\hline 7 & 2.098 & 145.00 & 61.95 & 0.00 & 0.00 & BB & 2.3407 \\
\hline 8 & 2.405 & 183.40 & 61.17 & 0.00 & 0.00 & BB & 2.9984 \\
\hline 9 & 2.506 & 151.60 & 89.16 & 0.00 & 0.00 & BB & 1.7003 \\
\hline 10 & 2.572 & 207.20 & 103.39 & 0.00 & 0.00 & BB & 2.0042 \\
\hline 11 & 2.640 & 101.20 & 41.18 & $9 e-05$ & $9 e-05$ & BB & 2.4576 \\
\hline 12 & 2.786 & 257.20 & 67.01 & 0.00 & 0.00 & BB & 3.8381 \\
\hline 13 & 2.849 & 200.35 & 112.91 & 0.00 & 0.00 & BV & 1.7744 \\
\hline 14 & 2.906 & 306.05 & 86.85 & 0.00 & 0.00 & VB & 3.5239 \\
\hline 15 & 2.990 & 98.20 & 75.11 & $9 e-05$ & $9 e-05$ & BB & 1.3074 \\
\hline 16 & 3.221 & 70.20 & 63.19 & $6 e-05$ & $6 e-05$ & BB & 1.1109 \\
\hline 17 & 3.705 & 196.00 & 94.34 & 0.00 & 0.00 & BB & 2.0777 \\
\hline 18 & 3.855 & 172.40 & 82.58 & 0.00 & 0.00 & BB & 2.0877 \\
\hline 19 & 3.986 & 613.82 & 90.56 & 0.00 & 0.00 & BV & 6.7781 \\
\hline 20 & 4.118 & 207.63 & 88.30 & 0.00 & 0.00 & VV & 2.3514 \\
\hline 21 & 4.192 & 301.02 & 83.40 & 0.00 & 0.00 & VV & 3.6095 \\
\hline 22 & 4.248 & 147.33 & 80.52 & 0.00 & 0.00 & VB & 1.8297 \\
\hline 23 & 4.300 & 76.00 & 66.24 & $7 e-05$ & $7 e-05$ & BB & 1.1473 \\
\hline 24 & 4.385 & 97.60 & 73.69 & $9 e-05$ & $9 e-05$ & $\mathrm{BB}$ & 1.3244 \\
\hline 25 & 4.658 & 475.60 & 113.03 & 0.00 & 0.00 & $\mathrm{BB}$ & 4.2078 \\
\hline 26 & 4.764 & 50.45 & 68.72 & $5 e-05$ & $5 e-05$ & BV & 0.7341 \\
\hline 27 & 4.792 & 453.55 & 80.92 & 0.00 & 0.00 & VB & 5.6051 \\
\hline 28 & 5.062 & 393.20 & 67.29 & 0.00 & 0.00 & BB & 5.8433 \\
\hline 29 & 5.321 & 455.60 & 89.48 & 0.00 & 0.00 & BB & 5.0915 \\
\hline 30 & 5.417 & 47.60 & 66.73 & $4 e-05$ & $4 \mathrm{e}-05$ & BB & 0.7133 \\
\hline 31 & 6.277 & 53455.53 & 8595.70 & 0.05 & 0.05 & BV & 6.2189 \\
\hline 32 & 6.583 & 5482159.97 & 223020.29 & 5.02 & 5.02 & VV & 24.5814 \\
\hline 33 & 7.293 & 3382643.22 & 92756.48 & 3.10 & 3.10 & VV & 36.4680 \\
\hline 34 & 8.183 & 903827.98 & 59424.96 & 0.83 & 0.83 & VV & 15.2096 \\
\hline 35 & 8.601 & 13702074.69 & 580668.14 & 12.55 & 12.55 & VV & 23.5971 \\
\hline 36 & 9.267 & 54703933.54 & 991695.74 & 50.10 & 50.10 & VE & 55.1620 \\
\hline 37 & 11.730 & 743485.20 & 12509.48 & 0.68 & 0.68 & EV & 59.4338 \\
\hline 38 & 13.358 & 137453.68 & 4187.75 & 0.13 & 0.13 & VB & 32.8228 \\
\hline 39 & 14.778 & 77.20 & 46.23 & $7 e-05$ & $7 e-05$ & BB & 1.6700 \\
\hline 40 & 15.336 & 16930.87 & 1272.04 & 0.02 & 0.02 & BV & 13.3100 \\
\hline 41 & 15.385 & 14869.33 & 1240.23 & 0.01 & 0.01 & VB & 11.9892 \\
\hline 42 & 15.965 & 250.40 & 96.88 & 0.00 & 0.00 & BB & 2.5846 \\
\hline 43 & 16.388 & 323.60 & 101.36 & 0.00 & 0.00 & BB & 3.1927 \\
\hline 44 & 16.955 & 68.40 & 56.59 & $6 e-05$ & $6 e-05$ & $\mathrm{BB}$ & 1.2086 \\
\hline 45 & 17.424 & 93.80 & 37.11 & $9 e-05$ & $9 e-05$ & $\mathrm{BB}$ & 16.5277 \\
\hline
\end{tabular}


6/21/2013 12:48:01 PM Result: c:Ihplc datalrobinlethanol_june 10-2013lethanol008.rst

\begin{tabular}{|c|c|c|c|c|c|c|c|}
\hline $\begin{array}{c}\text { Peak } \\
\#\end{array}$ & $\begin{array}{l}\text { Time } \\
\text { [min] }\end{array}$ & $\begin{array}{c}\text { Area } \\
{[\mu \mathrm{V} \cdot \mathrm{s}]}\end{array}$ & $\begin{array}{l}\text { Height } \\
{[\mu \mathrm{V}]}\end{array}$ & $\begin{array}{c}\text { Area } \\
{[\%]}\end{array}$ & $\begin{array}{c}\text { Norm. Area } \\
{[\%]}\end{array}$ & $B L$ & $\begin{array}{c}\text { Area/Height } \\
\text { [s] }\end{array}$ \\
\hline 46 & 17.523 & 100.80 & 49.41 & $9 e-05$ & $9 e-05$ & BB & 2.0402 \\
\hline 47 & 17.834 & 85.00 & 36.86 & $8 e-05$ & $8 e-05$ & $\mathrm{BB}$ & 2.3063 \\
\hline 48 & 17.918 & 201.09 & 83.50 & 0.00 & 0.00 & BV & 2.4081 \\
\hline 49 & 18.309 & 6651.11 & 417.28 & 0.01 & 0.01 & VB & 15.9391 \\
\hline 50 & 18.888 & 142.00 & 55.11 & 0.00 & 0.00 & $\mathrm{BB}$ & 2.5765 \\
\hline 51 & 19.300 & 584.40 & 45.27 & 0.00 & 0.00 & BB & 12.9099 \\
\hline 52 & 19.439 & 231.40 & 46.68 & 0.00 & 0.00 & $\mathrm{BB}$ & 4.9576 \\
\hline 53 & 20.077 & 155.42 & 59.99 & 0.00 & 0.00 & BV & 2.5909 \\
\hline 54 & 20.524 & 2688.23 & 180.16 & 0.00 & 0.00 & VV & 14.9209 \\
\hline 55 & 20.578 & 681.48 & 198.33 & 0.00 & 0.00 & VV & 3.4361 \\
\hline 56 & 21.539 & 30032187.67 & 753475.41 & 27.50 & 27.50 & VE & 39.8582 \\
\hline 57 & 23.291 & 773.20 & 82.90 & 0.00 & 0.00 & EB & 9.3275 \\
\hline 58 & 23.615 & 633.20 & 70.43 & 0.00 & 0.00 & $\mathrm{BB}$ & 8.9899 \\
\hline 59 & 23.771 & 372.60 & 90.04 & 0.00 & 0.00 & $\mathrm{BB}$ & 4.1381 \\
\hline 60 & 24.037 & 302.40 & 79.50 & 0.00 & 0.00 & BB & 3.8037 \\
\hline 61 & 24.210 & 33.60 & 34.85 & $3 e-05$ & $3 e-05$ & $\mathrm{BB}$ & 0.9640 \\
\hline 62 & 24.611 & 56.80 & 48.53 & $5 e-05$ & $5 e-05$ & $\mathrm{BB}$ & 1.1703 \\
\hline \multirow[t]{2}{*}{63} & 24.889 & 164.80 & 74.44 & 0.00 & 0.00 & $\mathrm{BB}$ & 2.2138 \\
\hline & & $1.09 e+08$ & $2.73 e+06$ & 100.00 & 100.00 & & \\
\hline
\end{tabular}

Warning -- Signal level out-of-range in peak

Missing Component Report

Component Expected Retention (Calibration File) 
Time of Injection: 6/11/2013 4:23:49 PM

Scale Factor: $1.0 \quad$ Plot Offset: $-42.86 \mathrm{mV}$

Low Point : $-42.86 \mathrm{mV}$

High Point : $1000.00 \mathrm{mV}$

Plot Scale: $1042.9 \mathrm{mV}$

Response [mV]

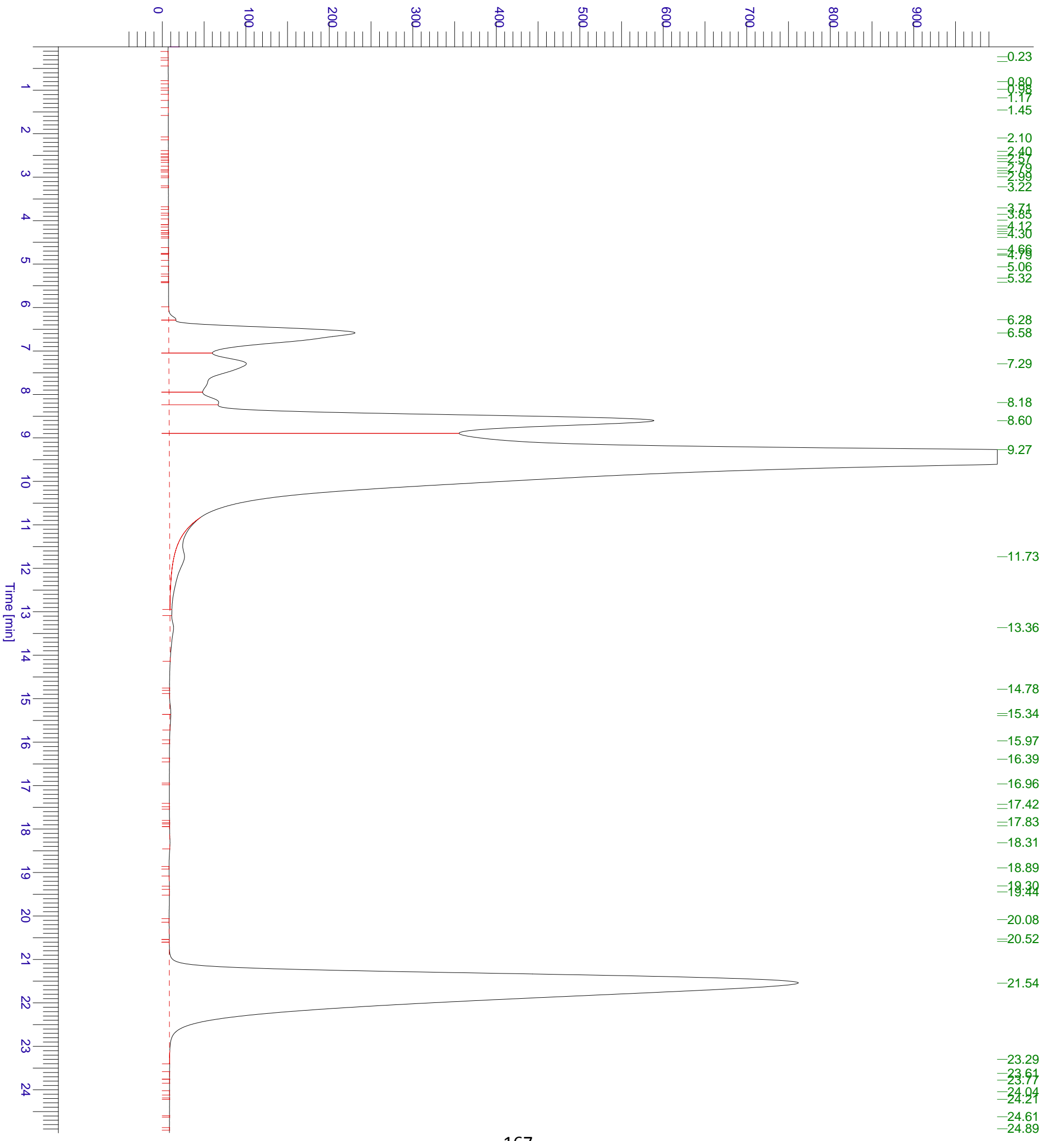




$\begin{array}{llll}\text { Software Version } & : 6.3 .2 .0646 & \text { Date } & : 6 / 21 / 201312: 57: 56 \text { PM } \\ \text { Operator } & : \text { rLuong } & \text { Sample Name } & : \text { S10 } \\ \text { Sample Number } & : \text { O17 } & \text { Study } & : \text { Ethanol } \\ \text { AutoSampler } & : \text { SER200 } & \text { Rack/Vial } & : 1 / 17 \\ \text { Instrument Name } & : \text { HPLC } & \text { Channel } & : \text { A } \\ \text { Instrument Serial \# } & : \text { None } & \text { A/D mV Range }: 1000 \\ \text { Delay Time } & : 0.00 \mathrm{~min} & \text { End Time } & : 24.99 \mathrm{~min} \\ \text { Sampling Rate } & : 2.5000 \mathrm{pts} / \mathrm{s} & & \\ \text { Sample Volume } & : 1.000000 \mathrm{ul} & & \\ \text { Sample Amount } & : 1.0000 & \text { Area Reject } & : 0.000000 \\ \text { Data Acquisition Time } & : 6 / 11 / 2013 \text { 8:20:13 PM } & \text { Dilution Factor }: 1.00\end{array}$

Raw Data File : C:IHPLC DatalrobinlEthanol_June 10-2013IEthanol017.raw

Result File : c:Ihplc datalrobinlethanol_june 10-2013lethanol017.rst [Editing in Progress]

Inst Method : C:IHPLC DatalrobinlMethod Robin 87H from C:IHPLC DatalrobinlEthanol_June 10-2013।Ethanol017.raw

Proc Method : C:IHPLC DatalrobinlMethod Robin 87H from c:Ihplc datalrobinlethanol_june 10-2013lethanol017.rst [Editing in Progress] Calib Method : C:IHPLC DatalrobinlMethod Robin 87H from c:Ihplc datalrobinlethanol_june 10-2013lethanol017.rst [Editing in Progress]

Report Format File: C:IHPLC DatalrobinlMethod Robin 87H.rpt

Sequence File : C:IHPLC DatalrobinIValera Sequence 87H-Etanol_June 10_2013.seq

\begin{tabular}{|c|c|c|c|c|c|c|c|}
\hline $\begin{array}{c}\text { Peak } \\
\quad \#\end{array}$ & $\begin{array}{l}\text { Time } \\
\text { [min] }\end{array}$ & $\begin{array}{l}\text { Area } \\
{[\mu \mathrm{V} \cdot \mathrm{s}]}\end{array}$ & $\begin{array}{l}\text { Height } \\
{[\mu \mathrm{V}]}\end{array}$ & $\begin{array}{c}\text { Area } \\
{[\%]}\end{array}$ & $\begin{array}{c}\text { Norm. Area } \\
{[\%]}\end{array}$ & $B L$ & $\begin{array}{c}\text { Area/Height } \\
\text { [s] }\end{array}$ \\
\hline - & 0.001 & 0.00 & 0.00 & 0.00 & 0.00 & & \\
\hline 1 & 0.025 & 84.40 & 50.71 & $8 e-05$ & $8 e-05$ & BB & 1.6645 \\
\hline 2 & 0.255 & 1339.60 & 143.84 & 0.00 & 0.00 & BB & 9.3129 \\
\hline 3 & 0.504 & 165.20 & 92.60 & 0.00 & 0.00 & BB & 1.7840 \\
\hline 4 & 0.731 & 205.20 & 38.58 & 0.00 & 0.00 & $\mathrm{BB}$ & 5.3184 \\
\hline 5 & 2.177 & 143.20 & 36.47 & 0.00 & 0.00 & BB & 3.9265 \\
\hline 6 & 2.499 & 135.80 & 32.16 & 0.00 & 0.00 & BB & 4.2229 \\
\hline 7 & 2.820 & 161.20 & 37.32 & 0.00 & 0.00 & BB & 4.3200 \\
\hline 8 & 3.145 & 207.27 & 38.91 & 0.00 & 0.00 & BV & 5.3271 \\
\hline 9 & 3.301 & 156.33 & 37.33 & 0.00 & 0.00 & VB & 4.1879 \\
\hline 10 & 3.463 & 72.00 & 26.47 & $7 e-05$ & $7 e-05$ & BB & 2.7201 \\
\hline 11 & 3.784 & 137.60 & 31.13 & 0.00 & 0.00 & BB & 4.4197 \\
\hline 12 & 4.106 & 148.00 & 33.66 & 0.00 & 0.00 & BB & 4.3975 \\
\hline 13 & 4.583 & 61.20 & 25.23 & $6 e-05$ & $6 e-05$ & BB & 2.4253 \\
\hline 14 & 6.568 & 3099266.35 & 109465.00 & 3.03 & 3.03 & BV & 28.3129 \\
\hline 15 & 7.303 & 3307798.33 & 90922.22 & 3.24 & 3.24 & VV & 36.3805 \\
\hline 16 & 8.610 & 17241122.16 & 691790.70 & 16.87 & 16.87 & VV & 225 \\
\hline 17 & 9.320 & 49904628.16 & 997531.66 & 48.83 & 48.83 & VB & 50.0281 \\
\hline 18 & 13.413 & 174493.60 & 14190.00 & 0.17 & 0.17 & BB & 12.2969 \\
\hline 19 & 13.718 & 675894.40 & 33875.01 & 0.66 & 0.66 & BB & 19.9526 \\
\hline 20 & 14.862 & 189.63 & 97.37 & 0.00 & 0.00 & BV & 1.9475 \\
\hline 21 & 15.369 & 634771.97 & 19884.35 & 0.62 & 0.62 & VB & 31.9232 \\
\hline 22 & 16.615 & 244.40 & 50.88 & 0.00 & 0.00 & BB & 4.8039 \\
\hline 23 & 16.779 & 157.20 & 84.93 & 0.00 & 0.00 & BB & 1.8510 \\
\hline 24 & 16.910 & 66.00 & 41.40 & $6 e-05$ & $6 e-05$ & BB & 1.5943 \\
\hline 25 & 17.010 & 179.20 & 81.71 & 0.00 & 0.00 & BB & 2.1931 \\
\hline 26 & 17.256 & 356.80 & 130.37 & 0.00 & 0.00 & BB & 2.7368 \\
\hline 27 & 17.510 & 199.40 & 78.66 & 0.00 & 0.00 & BB & 2.5349 \\
\hline 28 & 17.577 & 163.20 & 76.15 & 0.00 & 0.00 & BB & 2.1431 \\
\hline 29 & 17.802 & 563.67 & 129.40 & 0.00 & 0.00 & $\mathrm{BV}$ & 4.3560 \\
\hline 30 & 17.922 & 1918.37 & 220.97 & 0.00 & 0.00 & VV & 8.6816 \\
\hline 31 & 18.029 & 512.19 & 281.15 & 0.00 & 0.00 & VV & 1.8218 \\
\hline 32 & 18.094 & 2847.28 & 399.97 & 0.00 & 0.00 & VV & 7.1188 \\
\hline 33 & 18.212 & 3075.29 & 362.83 & 0.00 & 0.00 & VB & 8.4758 \\
\hline 34 & 18.763 & 79.20 & 62.23 & $8 e-05$ & $8 e-05$ & BB & 1.2728 \\
\hline 35 & 19.286 & 98856.52 & 2921.24 & 0.10 & 0.10 & $\mathrm{BE}$ & 33.8406 \\
\hline 36 & 19.919 & 788.80 & 104.83 & 0.00 & 0.00 & EV & 7.5248 \\
\hline 37 & 20.030 & 322.28 & 149.18 & 0.00 & 0.00 & VB & 2.1603 \\
\hline 38 & 20.084 & 127.60 & 92.00 & 0.00 & 0.00 & BB & 1.3870 \\
\hline 39 & 20.196 & 131.20 & 104.86 & 0.00 & 0.00 & BB & 1.2512 \\
\hline 40 & 20.234 & 372.40 & 103.06 & 0.00 & 0.00 & BB & 3.6133 \\
\hline 41 & 20.314 & 131.00 & 134.02 & 0.00 & 0.00 & $\mathrm{BB}$ & 0.9775 \\
\hline 42 & 20.483 & 258.80 & 100.34 & 0.00 & 0.00 & BB & 2.5793 \\
\hline 43 & 20.531 & 298.40 & 152.42 & 0.00 & 0.00 & BB & 1.9577 \\
\hline 44 & 20.602 & 94.60 & 98.32 & $9 e-05$ & $9 e-05$ & BB & 0.9622 \\
\hline 45 & 20.637 & 409.67 & 131.79 & 0.00 & 0.00 & BV & 16.9085 \\
\hline
\end{tabular}


6/21/2013 12:57:56 PM Result: c:Ihplc datalrobinlethanol_june 10-2013lethanol017.rst

\begin{tabular}{|c|c|c|c|c|c|c|c|}
\hline $\begin{array}{c}\text { Peak } \\
\quad \#\end{array}$ & $\begin{array}{l}\text { Time } \\
\text { [min] }\end{array}$ & $\begin{array}{c}\text { Area } \\
{[\mu \mathrm{V} \cdot \mathrm{s}]}\end{array}$ & $\begin{array}{l}\text { Height } \\
{[\mu \mathrm{V}]}\end{array}$ & $\begin{array}{c}\text { Area } \\
{[\%]}\end{array}$ & $\begin{array}{c}\text { Norm. Area } \\
{[\%]}\end{array}$ & $B L$ & $\begin{array}{c}\text { Area/Height } \\
\text { [s] }\end{array}$ \\
\hline 46 & 20.762 & 808.98 & 266.36 & 0.00 & 0.00 & VV & 3.0372 \\
\hline 47 & 20.806 & 613.24 & 337.95 & 0.00 & 0.00 & VV & 1.8146 \\
\hline 48 & 21.538 & 27048584.91 & 682579.76 & 26.46 & 26.46 & VE & 39.6270 \\
\hline 49 & 22.877 & 668.40 & 142.44 & 0.00 & 0.00 & EB & 4.6924 \\
\hline 50 & 23.199 & 348.40 & 99.94 & 0.00 & 0.00 & BB & 3.4860 \\
\hline 51 & 23.311 & 47.20 & 66.32 & $5 e-05$ & $5 e-05$ & $\mathrm{BB}$ & 0.7117 \\
\hline 52 & 23.364 & 141.20 & 59.72 & 0.00 & 0.00 & BB & 2.3644 \\
\hline 53 & 23.502 & 453.54 & 144.48 & 0.00 & 0.00 & BV & 3.1390 \\
\hline 54 & 23.575 & 188.57 & 129.51 & 0.00 & 0.00 & VV & 1.4560 \\
\hline 55 & 23.618 & 117.89 & 98.91 & 0.00 & 0.00 & VB & 1.1919 \\
\hline 56 & 23.665 & 58.00 & 79.18 & $6 e-05$ & $6 e-05$ & BB & 0.7325 \\
\hline 57 & 24.031 & 416.00 & 160.43 & 0.00 & 0.00 & BB & 2.5930 \\
\hline 58 & 24.146 & 36.40 & 49.41 & $4 e-05$ & $4 e-05$ & BB & 0.7367 \\
\hline 59 & 24.221 & 203.20 & 102.58 & 0.00 & 0.00 & BB & 1.9809 \\
\hline 60 & 24.424 & 1149.00 & 179.38 & 0.00 & 0.00 & BV & 6.4055 \\
\hline 61 & 24.558 & 397.40 & 129.82 & 0.00 & 0.00 & VB & 3.0613 \\
\hline 62 & 24.647 & 205.60 & 103.30 & 0.00 & 0.00 & BB & 1.9903 \\
\hline 63 & 24.830 & 504.80 & 68.88 & 0.00 & 0.00 & BB & 7.3284 \\
\hline \multirow[t]{2}{*}{64} & 24.974 & 108.40 & 58.59 & 0.00 & 0.00 & BB & 1.8502 \\
\hline & & 1.02 & 2.65 & 0.00 & 100.00 & & \\
\hline
\end{tabular}

Warning -- Signal level out-of-range in peak

Missing Component Report

Component Expected Retention (Calibration File)

0.001 
Time of Injection: 6/11/2013 8:20:13 PM

Scale Factor: $1.0 \quad$ Plot Offset: $-50.00 \mathrm{mV}$

Low Point : $-50.00 \mathrm{mV}$

High Point : $1000.00 \mathrm{mV}$

Plot Scale: $1050.0 \mathrm{mV}$

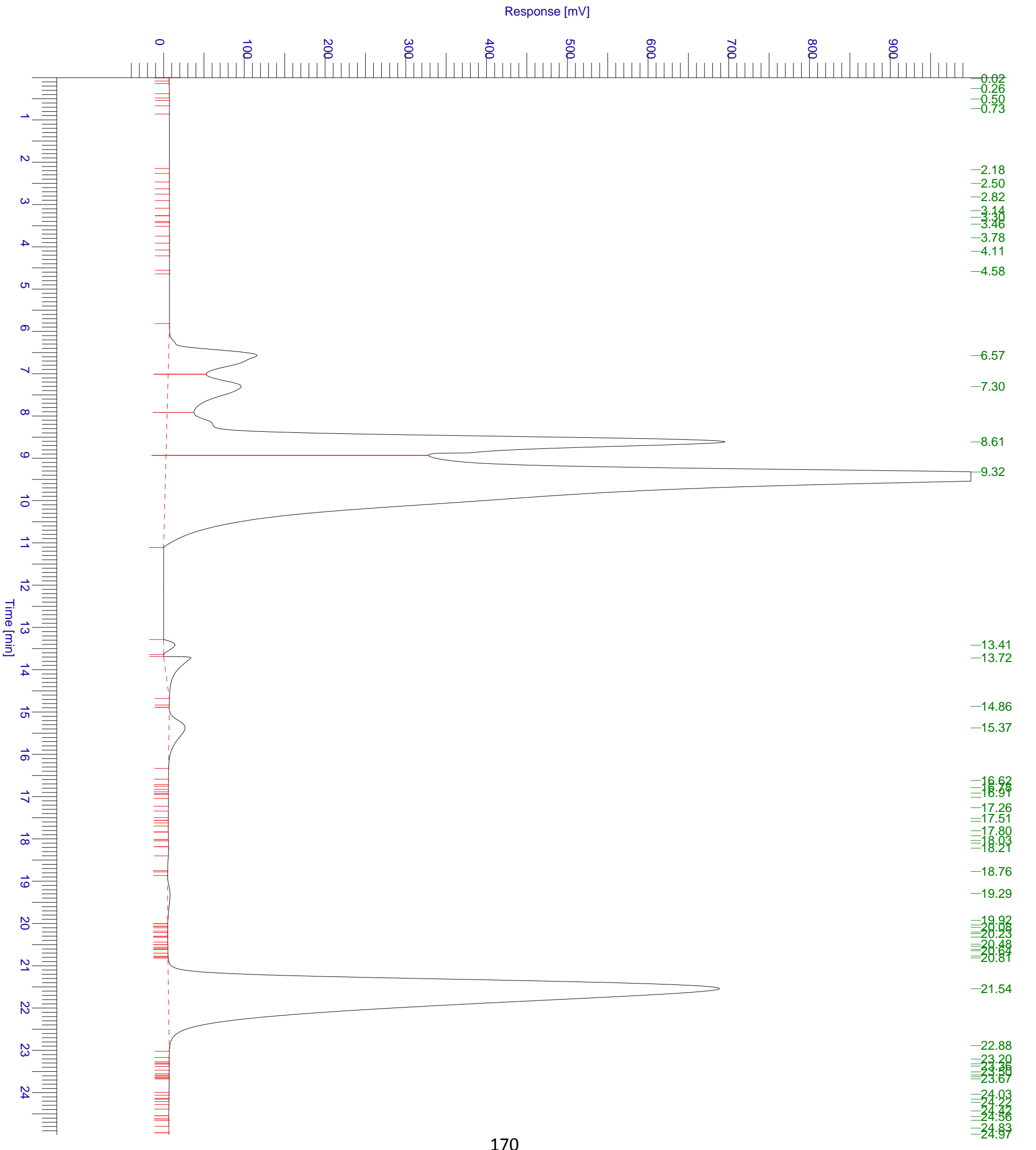




$\begin{array}{llll}\text { Software Version } & : 6.3 .2 .0646 & \text { Date } & : 6 / 21 / 201312: 51: 08 \text { PM } \\ \text { Operator } & : \text { rLuong } & \text { Sample Name } & : \text { S2 } \\ \text { Sample Number } & : \text { O09 } & \text { Study } & : \text { Ethanol } \\ \text { AutoSampler } & : \text { SER200 } & \text { Rack/Vial } & : 1 / 9 \\ \text { Instrument Name } & : \text { HPLC } & \text { Channel } & : \text { A } \\ \text { Instrument Serial \# } & : \text { None } & \text { A/D mV Range }: 1000 \\ \text { Delay Time } & : 0.00 \mathrm{~min} & \text { End Time } & : 24.99 \mathrm{~min} \\ \text { Sampling Rate } & : 2.5000 \mathrm{pts} / \mathrm{s} & & \\ \text { Sample Volume } & : 1.000000 \mathrm{ul} & & \\ \text { Sample Amount } & : 1.0000 & \text { Area Reject } & : 0.000000 \\ \text { Data Acquisition Time } & : 6 / 11 / 2013 \text { 4:50:06 PM } & \text { Dilution Factor }: 1.00\end{array}$

Raw Data File : C:IHPLC DatalrobinlEthanol_June 10-2013IEthanol009.raw

Result File : c:Ihplc datalrobinlethanol_june 10-2013lethanol009.rst [Editing in Progress]

Inst Method : C:IHPLC DatalrobinlMethod Robin 87H from C:IHPLC DatalrobinlEthanol_June 10-2013।Ethanol009.raw

Proc Method : C:IHPLC DatalrobinlMethod Robin 87H from c:Ihplc datalrobinlethanol_june 10-2013lethanol009.rst [Editing in Progress] Calib Method : C:IHPLC DatalrobinlMethod Robin 87H from c:Ihplc datalrobinlethanol_june 10-2013lethanol009.rst [Editing in Progress]

Report Format File: C:IHPLC DatalrobinlMethod Robin 87H.rpt

Sequence File : C:IHPLC DatalrobinIValera Sequence 87H-Etanol_June 10_2013.seq

\begin{tabular}{|c|c|c|c|c|c|c|c|}
\hline $\begin{array}{l}\text { Peak } \\
\quad \#\end{array}$ & $\begin{array}{l}\text { Time } \\
\text { [min] }\end{array}$ & $\begin{array}{l}\text { Area } \\
{[\mu \mathrm{V} \cdot \mathrm{s}]}\end{array}$ & $\begin{array}{l}\text { Height } \\
{[\mu \mathrm{V}]}\end{array}$ & $\begin{array}{c}\text { Area } \\
{[\%]}\end{array}$ & $\begin{array}{c}\text { Norm. Area } \\
{[\%]}\end{array}$ & $\mathrm{BL}$ & $\begin{array}{c}\text { Area/Height } \\
{[\mathrm{s}]}\end{array}$ \\
\hline- & 0.001 & 0.00 & 0.00 & 0.00 & 0.00 & & \\
\hline 1 & 0.196 & 132.80 & 53.94 & 0.00 & 0.00 & BB & 2.4622 \\
\hline 2 & 0.470 & 148.20 & 73.60 & 0.00 & 0.00 & BB & 2.0136 \\
\hline 3 & 0.616 & 123.20 & 58.11 & 0.00 & 0.00 & BB & 2.1199 \\
\hline 4 & 0.884 & 222.00 & 62.81 & 0.00 & 0.00 & BB & 3.5342 \\
\hline 5 & 1.033 & 524.34 & 90.07 & 0.00 & 0.00 & $\mathrm{BV}$ & 5.8215 \\
\hline 6 & 1.204 & 558.08 & 85.80 & 0.00 & 0.00 & VV & 6.5044 \\
\hline 7 & 1.370 & 170.78 & 66.04 & 0.00 & 0.00 & VB & 2.5859 \\
\hline 8 & 1.618 & 151.80 & 64.94 & 0.00 & 0.00 & BB & 2.3375 \\
\hline 9 & 1.769 & 440.80 & 58.03 & 0.00 & 0.00 & BB & 7.5965 \\
\hline 10 & 1.923 & 186.00 & 93.92 & 0.00 & 0.00 & BB & 1.9805 \\
\hline 11 & 2.351 & 184.00 & 60.86 & 0.00 & 0.00 & BB & 3.0231 \\
\hline 12 & 2.715 & 68.40 & 66.70 & $6 e-05$ & $6 e-05$ & BB & 1.0254 \\
\hline 13 & 2.803 & 167.60 & 49.92 & 0.00 & 0.00 & BB & 3.3573 \\
\hline 14 & 3.255 & 151.22 & 74.26 & 0.00 & 0.00 & BV & 2.0363 \\
\hline 15 & 3.295 & 309.58 & 74.78 & 0.00 & 0.00 & VB & 4.1397 \\
\hline 16 & 3.453 & 359.60 & 81.15 & 0.00 & 0.00 & BB & 4.4313 \\
\hline 17 & 3.646 & 396.80 & 76.80 & 0.00 & 0.00 & $\mathrm{BB}$ & 5.1668 \\
\hline 18 & 4.062 & 62.20 & 44.30 & $5 e-05$ & $5 e-05$ & BB & 1.4042 \\
\hline 19 & 4.424 & 86.00 & 42.19 & $7 e-05$ & $7 e-05$ & BB & 2.0382 \\
\hline 20 & 4.756 & 213.60 & 88.53 & 0.00 & 0.00 & BB & 2.4127 \\
\hline 21 & 5.386 & 100.20 & 38.02 & $9 e-05$ & 9e-05 & BB & 2.6355 \\
\hline 22 & 5.472 & 116.00 & 69.26 & $1 \mathrm{e}-04$ & $1 e-04$ & BB & 1.6749 \\
\hline 23 & 5.703 & 51.00 & 43.55 & $4 e-05$ & $4 e-05$ & BB & 1.1710 \\
\hline 24 & 6.263 & 37642.49 & 5077.80 & 0.03 & 0.03 & BV & 7.4131 \\
\hline 25 & 6.594 & 5922086.07 & 242086.60 & 5.05 & 5.05 & VV & 24.4627 \\
\hline 26 & 7.304 & 3660796.23 & 100751.52 & 3.12 & 3.12 & VV & 36.3349 \\
\hline 27 & 8.195 & 948288.87 & 63714.40 & 0.81 & 0.81 & VV & 14.8834 \\
\hline 28 & 8.611 & 15108230.12 & 639383.55 & 12.89 & 12.89 & VV & 23.6294 \\
\hline 29 & 9.273 & 57923422.62 & 961605.23 & 49.41 & 49.41 & VE & 60.2362 \\
\hline 30 & 11.757 & 492638.40 & 10507.86 & 0.42 & 0.42 & EB & 46.8828 \\
\hline 31 & 13.088 & 593.22 & 142.16 & 0.00 & 0.00 & BV & 4.1730 \\
\hline 32 & 13.401 & 63354.38 & 2573.13 & 0.05 & 0.05 & VB & 24.6215 \\
\hline 33 & 14.369 & 445.80 & 87.21 & 0.00 & 0.00 & BB & 5.1120 \\
\hline 34 & 15.376 & 130015.00 & 4480.87 & 0.11 & 0.11 & BB & 29.0156 \\
\hline 35 & 16.698 & 185.20 & 58.88 & 0.00 & 0.00 & BB & 3.1452 \\
\hline 36 & 17.438 & 138.40 & 56.88 & 0.00 & 0.00 & BB & 2.4332 \\
\hline 37 & 18.306 & 9123.60 & 274.10 & 0.01 & 0.01 & BB & 33.2862 \\
\hline 38 & 19.253 & 266.40 & 51.03 & 0.00 & 0.00 & $\mathrm{BB}$ & 5.2202 \\
\hline 39 & 20.408 & 1098.53 & 129.09 & 0.00 & 0.00 & BV & 8.5097 \\
\hline 40 & 20.540 & 925.59 & 168.73 & 0.00 & 0.00 & VV & 5.4855 \\
\hline 41 & 21.543 & 32922225.82 & 819672.06 & 28.08 & 28.08 & VB & 40.1651 \\
\hline 42 & 23.366 & 179.20 & 88.03 & 0.00 & 0.00 & $\mathrm{BB}$ & 2.0358 \\
\hline 43 & 23.518 & 318.80 & 79.63 & 0.00 & 0.00 & BB & 4.0035 \\
\hline 44 & 23.650 & 507.20 & 74.42 & 0.00 & 0.00 & BB & 6.814 \\
\hline 45 & 24.013 & 201.52 & 93.35 & 0.00 & 0.00 & BV & $12 . \pm 586$ \\
\hline
\end{tabular}


6/21/2013 12:51:08 PM Result: c:Ihplc datalrobinlethanol_june 10-2013lethanol009.rst

\begin{tabular}{|c|c|c|c|c|c|c|c|}
\hline $\begin{array}{c}\text { Peak } \\
\#\end{array}$ & $\begin{array}{l}\text { Time } \\
\text { [min] }\end{array}$ & $\begin{array}{l}\text { Area } \\
{[\mu \mathrm{V} \cdot \mathrm{s}]}\end{array}$ & $\begin{array}{l}\text { Height } \\
{[\mu \mathrm{V}]}\end{array}$ & $\begin{array}{c}\text { Area } \\
{[\%]}\end{array}$ & $\begin{array}{c}\text { Norm. Area } \\
{[\%]}\end{array}$ & $\mathrm{BL}$ & $\begin{array}{c}\text { Area/Height } \\
\text { [s] }\end{array}$ \\
\hline 46 & 24.070 & 193.08 & 71.76 & 0.00 & 0.00 & VB & 2.6908 \\
\hline 47 & 24.226 & 125.00 & 60.99 & 0.00 & 0.00 & BB & 2.0496 \\
\hline 48 & 24.667 & 324.80 & 76.91 & 0.00 & 0.00 & BB & 4.2229 \\
\hline 49 & 24.824 & 125.60 & 63.98 & 0.00 & 0.00 & BB & 1.9632 \\
\hline \multirow[t]{2}{*}{50} & 24.976 & 50.80 & 36.68 & $4 e-05$ & $4 e-05$ & BB & 1.3851 \\
\hline & & $1.17 \mathrm{e}+08$ & $2.85 e+06$ & 100.00 & 100.00 & & \\
\hline
\end{tabular}

Warning -- Signal level out-of-range in peak

Missing Component Report

Component Expected Retention (Calibration File)

standards

0.001 
Time of Injection: 6/11/2013 4:50:06 PM

Start Time : $0.00 \mathrm{~min} \quad$ End Time : $24.99 \mathrm{~min} \quad$ Low Point : $-43.12 \mathrm{mV}$

Scale Factor: $1.0 \quad$ Plot Offset: $-43.12 \mathrm{mV}$

Plot Scale: $1043.1 \mathrm{mV}$

High Point : $1000.00 \mathrm{mV}$

Response [mV]

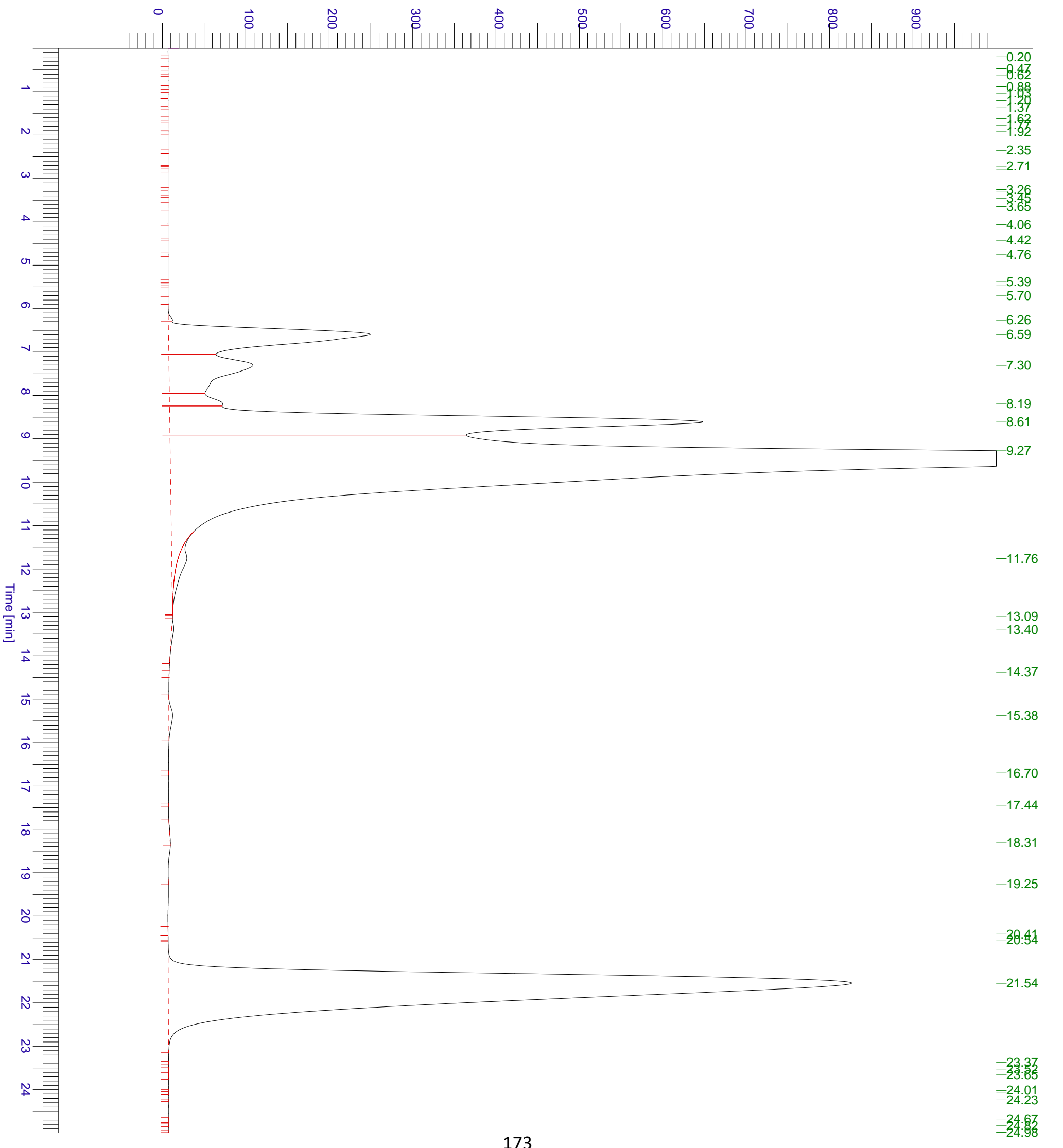




$\begin{array}{llll}\text { Software Version } & : 6.3 .2 .0646 & \text { Date } & : 6 / 21 / 201312: 51: 55 \text { PM } \\ \text { Operator } & : \text { rLuong } & \text { Sample Name } & : \text { S3 } \\ \text { Sample Number } & : \text { O10 } & \text { Study } & : \text { Ethanol } \\ \text { AutoSampler } & : \text { SER200 } & \text { Rack/Vial } & : 1 / 10 \\ \text { Instrument Name } & : \text { HPLC } & \text { Channel } & : \text { A } \\ \text { Instrument Serial \# } & : \text { None } & \text { A/D mV Range }: 1000 \\ \text { Delay Time } & : 0.00 \mathrm{~min} & \text { End Time } & : 24.99 \mathrm{~min} \\ \text { Sampling Rate } & : 2.5000 \mathrm{pts} / \mathrm{s} & & \\ \text { Sample Volume } & : 1.000000 \mathrm{ul} & & \\ \text { Sample Amount } & : 1.0000 & \text { Area Reject } & : 0.000000 \\ \text { Data Acquisition Time } & : 6 / 11 / 20135: 16: 22 \mathrm{PM} & \text { Dilution Factor }: 1.00\end{array}$

Raw Data File : C:IHPLC DatalrobinlEthanol_June 10-2013IEthanol010.raw

Result File : c:Ihplc datalrobinlethanol_june 10-2013lethanol010.rst [Editing in Progress]

Inst Method : C:IHPLC DatalrobinlMethod Robin 87H from C:IHPLC DatalrobinlEthanol_June 10-2013।Ethanol010.raw

Proc Method : C:IHPLC DatalrobinlMethod Robin 87H from c:Ihplc datalrobinlethanol_june 10-2013lethanol010.rst [Editing in Progress] Calib Method : C:IHPLC DatalrobinlMethod Robin 87H from c:Ihplc datalrobinlethanol_june 10-2013lethanol010.rst [Editing in Progress]

Report Format File: C:IHPLC DatalrobinlMethod Robin 87H.rpt

Sequence File : C:IHPLC DatalrobinIValera Sequence 87H-Etanol_June 10_2013.seq

\begin{tabular}{|c|c|c|c|c|c|c|c|}
\hline $\begin{array}{l}\text { Peak } \\
\quad \#\end{array}$ & $\begin{array}{l}\text { Time } \\
\text { [min] }\end{array}$ & $\begin{array}{l}\text { Area } \\
{[\mu \mathrm{V} \cdot \mathrm{s}]}\end{array}$ & $\begin{array}{l}\text { Height } \\
{[\mu \mathrm{V}]}\end{array}$ & $\begin{array}{c}\text { Area } \\
{[\%]}\end{array}$ & $\begin{array}{c}\text { Norm. Area } \\
{[\%]}\end{array}$ & $B L$ & $\begin{array}{c}\text { Area/Height } \\
{[\mathrm{s}]}\end{array}$ \\
\hline - & 0.001 & 0.00 & 0.00 & 0.00 & 0.00 & & \\
\hline 1 & 0.193 & 265.20 & 77.33 & 0.00 & 0.00 & BB & 3.4295 \\
\hline 2 & 0.312 & 140.40 & 78.44 & 0.00 & 0.00 & BB & 1.7899 \\
\hline 3 & 0.480 & 368.00 & 105.95 & 0.00 & 0.00 & BB & 3.4735 \\
\hline 4 & 0.561 & 54.20 & 46.01 & $5 e-05$ & $5 e-05$ & BB & 1.1780 \\
\hline 5 & 0.647 & 215.60 & 55.33 & 0.00 & 0.00 & BB & 3.8969 \\
\hline 6 & 0.768 & 91.47 & 82.44 & $8 e-05$ & $8 e-05$ & BV & 1.1096 \\
\hline 7 & 0.824 & 264.33 & 79.48 & 0.00 & 0.00 & VB & 3.3257 \\
\hline 8 & 1.013 & 140.20 & 56.55 & 0.00 & 0.00 & BB & 2.4792 \\
\hline 9 & 1.630 & 390.40 & 93.68 & 0.00 & 0.00 & BB & 4.1675 \\
\hline 10 & 1.762 & 126.00 & 54.84 & 0.00 & 0.00 & BB & 2.2975 \\
\hline 11 & 1.838 & 194.00 & 68.06 & 0.00 & 0.00 & BB & 2.8504 \\
\hline 12 & 2.074 & 493.80 & 109.35 & 0.00 & 0.00 & BB & 4.5159 \\
\hline 13 & 2.326 & 385.70 & 57.08 & 0.00 & 0.00 & BV & 6.7576 \\
\hline 14 & 2.404 & 359.90 & 92.18 & 0.00 & 0.00 & VB & 3.9044 \\
\hline 15 & 2.570 & 105.20 & 55.68 & $9 e-05$ & $9 e-05$ & BB & 1.8895 \\
\hline 16 & 2.720 & 468.00 & 50.52 & 0.00 & 0.00 & BB & 9.2646 \\
\hline 17 & 3.048 & 172.80 & 72.57 & 0.00 & 0.00 & $\mathrm{BB}$ & 2.3811 \\
\hline 18 & 4.164 & 79.00 & 39.39 & $7 e-05$ & $7 e-05$ & BB & 2.0055 \\
\hline 19 & 4.349 & 115.20 & 50.25 & 0.00 & 0.00 & BB & 2.2925 \\
\hline 20 & 5.463 & 44.60 & 42.31 & $4 e-05$ & $4 e-05$ & BB & 1.0541 \\
\hline 21 & 6.254 & 36847.27 & 4874.22 & 0.03 & 0.03 & BV & 7.5596 \\
\hline 22 & 6.581 & 5524360.03 & 227711.62 & 4.94 & 4.94 & VV & 24.2603 \\
\hline 23 & 7.292 & 3298351.72 & 95081.92 & 2.95 & 2.95 & VV & 34.6896 \\
\hline 24 & 8.187 & 897875.38 & 58892.62 & 0.80 & 0.80 & VV & 15.2460 \\
\hline 25 & 8.597 & 14275177.86 & 612837.28 & 12.75 & 12.75 & VV & 23.2936 \\
\hline 26 & 9.280 & 55726091.74 & 991821.24 & 49.79 & 49.79 & VE & 56.1856 \\
\hline 27 & 11.710 & 345557.60 & 7798.38 & 0.31 & 0.31 & EB & 44.3114 \\
\hline 28 & 13.001 & 501.16 & 124.66 & 0.00 & 0.00 & $\mathrm{BV}$ & 4.0202 \\
\hline 29 & 13.392 & 385057.64 & 13678.16 & 0.34 & 0.34 & VB & 28.1513 \\
\hline 30 & 14.770 & 187.93 & 73.55 & 0.00 & 0.00 & BV & 2.5550 \\
\hline 31 & 15.362 & 365650.26 & 11778.82 & 0.33 & 0.33 & VB & 31.0430 \\
\hline 32 & 16.857 & 312.40 & 53.36 & 0.00 & 0.00 & BB & 5.8549 \\
\hline 33 & 17.189 & 195.00 & 78.79 & 0.00 & 0.00 & $\mathrm{BB}$ & 2.4750 \\
\hline 34 & 17.357 & 152.51 & 56.68 & 0.00 & 0.00 & BV & 2.6905 \\
\hline 35 & 18.308 & 81863.23 & 2136.11 & 0.07 & 0.07 & VB & 38.3236 \\
\hline 36 & 18.895 & 193.01 & 90.51 & 0.00 & 0.00 & BV & 2.1325 \\
\hline 37 & 19.239 & 14003.58 & 1473.50 & 0.01 & 0.01 & VV & 9.5036 \\
\hline 38 & 19.298 & 11977.44 & 1564.08 & 0.01 & 0.01 & VV & 7.6578 \\
\hline 39 & 19.392 & 25278.17 & 1531.96 & 0.02 & 0.02 & VB & 16.5006 \\
\hline 40 & 19.962 & 98.40 & 41.23 & $9 e-05$ & $9 e-05$ & BB & 2.3866 \\
\hline 41 & 20.021 & 111.40 & 48.79 & $1 e-04$ & $1 e-04$ & BB & 2.2835 \\
\hline 42 & 20.140 & 63.60 & 55.08 & $6 e-05$ & $6 e-05$ & BB & 1.1547 \\
\hline 43 & 20.238 & 356.32 & 79.98 & 0.00 & 0.00 & BV & 4.4552 \\
\hline 44 & 20.387 & 465.73 & 78.03 & 0.00 & 0.00 & VV & 5.9686 \\
\hline 45 & 20.486 & 263.33 & 74.55 & 0.00 & 0.00 & VV & 17.5323 \\
\hline
\end{tabular}


6/21/2013 12:51:55 PM Result: c:Ihplc datalrobinlethanol_june 10-2013lethanol010.rst

\begin{tabular}{|c|c|c|c|c|c|c|c|}
\hline $\begin{array}{c}\text { Peak } \\
\quad \#\end{array}$ & $\begin{array}{l}\text { Time } \\
\text { [min] }\end{array}$ & $\begin{array}{l}\text { Area } \\
{[\mu \mathrm{V} \cdot \mathrm{s}]}\end{array}$ & $\begin{array}{l}\text { Height } \\
{[\mu \mathrm{V}]}\end{array}$ & $\begin{array}{c}\text { Area } \\
{[\%]}\end{array}$ & $\begin{array}{c}\text { Norm. Area } \\
{[\%]}\end{array}$ & $B L$ & $\begin{array}{c}\text { Area/Height } \\
{[\mathrm{s}]}\end{array}$ \\
\hline 46 & 20.603 & 205.82 & 14.58 & 0.00 & 0.00 & VB & 14.1177 \\
\hline 47 & 20.721 & 166.98 & 85.82 & 0.00 & 0.00 & BV & 1.9456 \\
\hline 48 & 21.531 & 30922998.22 & 774537.66 & 27.63 & 27.63 & VB & 39.9245 \\
\hline 49 & 23.451 & 166.60 & 79.06 & 0.00 & 0.00 & BB & 2.1073 \\
\hline 50 & 23.493 & 280.80 & 99.53 & 0.00 & 0.00 & BB & 2.8213 \\
\hline 51 & 23.710 & 138.80 & 51.20 & 0.00 & 0.00 & BB & 2.7109 \\
\hline 52 & 23.900 & 45.77 & 52.73 & $4 e-05$ & $4 e-05$ & BV & 0.8679 \\
\hline 53 & 23.931 & 372.63 & 131.75 & 0.00 & 0.00 & VB & 2.8283 \\
\hline 54 & 24.105 & 115.60 & 112.55 & 0.00 & 0.00 & BB & 1.0271 \\
\hline 55 & 24.168 & 68.40 & 52.57 & $6 e-05$ & $6 e-05$ & BB & 1.3012 \\
\hline 56 & 24.261 & 263.20 & 55.97 & 0.00 & 0.00 & BB & 4.7026 \\
\hline 57 & 24.439 & 500.20 & 93.43 & 0.00 & 0.00 & BB & 5.3537 \\
\hline 58 & 24.615 & 67.76 & 67.13 & $6 e-05$ & $6 e-05$ & BV & 1.0094 \\
\hline 59 & 24.641 & 54.72 & 67.06 & $5 e-05$ & $5 e-05$ & VV & 0.8160 \\
\hline 60 & 24.739 & 552.43 & 84.68 & 0.00 & 0.00 & VB & 6.5236 \\
\hline \multirow[t]{2}{*}{61} & 24.909 & 230.80 & 110.69 & 0.00 & 0.00 & BB & 2.0851 \\
\hline & & $1.12 \mathrm{e}+08$ & $2.81 e+06$ & 0.00 & 100.00 & & \\
\hline
\end{tabular}

Warning -- Signal level out-of-range in peak

Missing Component Report

Component Expected Retention (Calibration File)

standards

0.001 
Time of Injection: 6/11/2013 5:16:22 PM

Start Time : $0.00 \mathrm{~min} \quad$ End Time : $24.99 \mathrm{~min}$

Scale Factor: 1.0

Plot Offset: $-43.39 \mathrm{mV}$

Low Point : $-43.39 \mathrm{mV}$

High Point : $1000.00 \mathrm{mV}$

Plot Scale: $1043.4 \mathrm{mV}$

Response [mV]

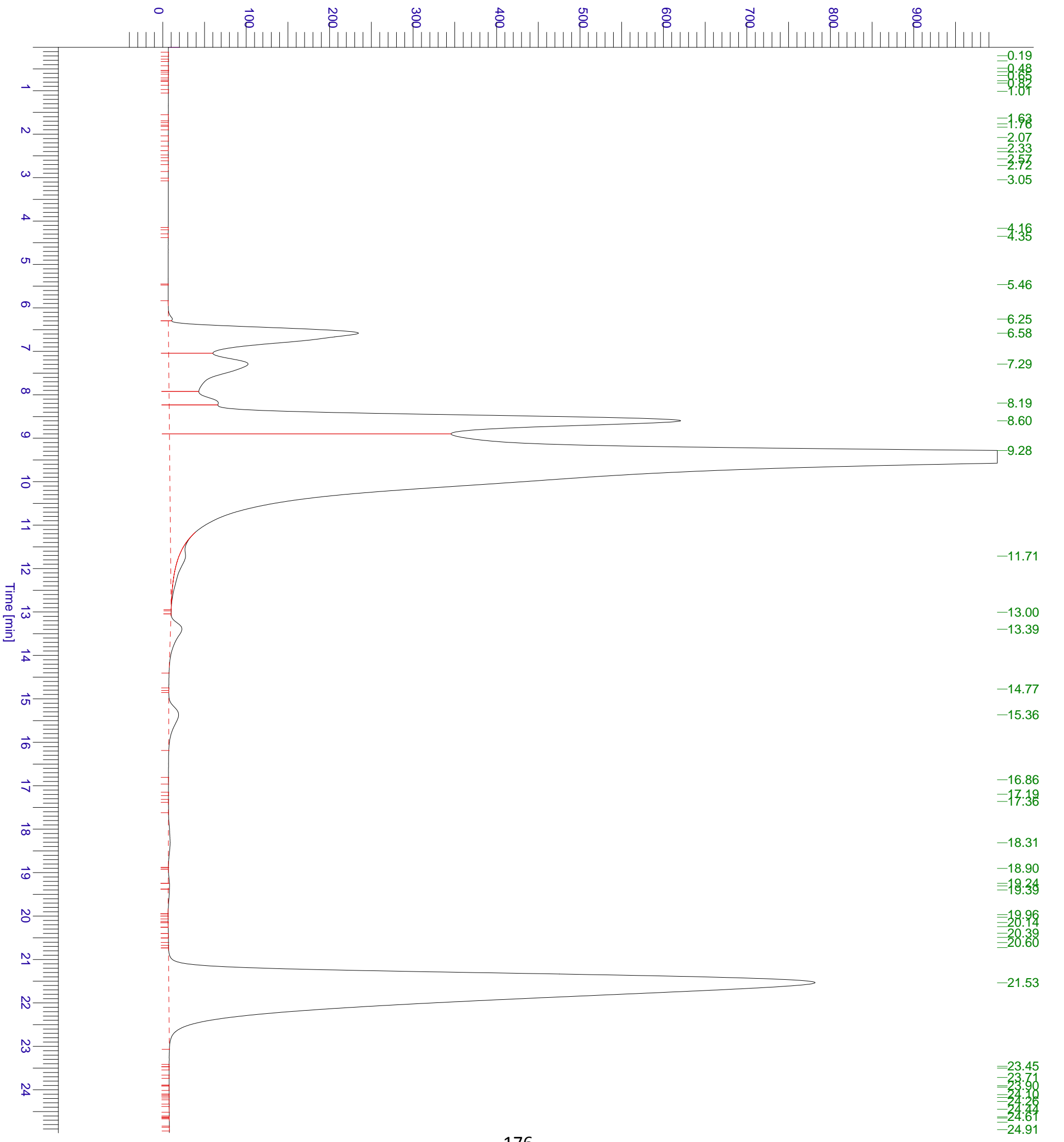




$\begin{array}{llll}\text { Software Version } & : 6.3 .2 .0646 & \text { Date } & : 6 / 21 / 2013 \text { 12:53:00 PM } \\ \text { Operator } & : \text { rLuong } & \text { Sample Name }: \text { S4 } \\ \text { Sample Number } & : \text { O11 } & \text { Study } & : \text { Ethanol } \\ \text { AutoSampler } & : \text { SER200 } & \text { Rack/Vial } & : 1 / 11 \\ \text { Instrument Name } & : \text { HPLC } & \text { Channel } & : \text { A } \\ \text { Instrument Serial \# } & : \text { None } & \text { A/D mV Range }: 1000 \\ \text { Delay Time } & : 0.00 \mathrm{~min} & \text { End Time } & : 24.99 \mathrm{~min} \\ \text { Sampling Rate } & : 2.5000 \mathrm{pts} / \mathrm{s} & & \\ \text { Sample Volume } & : 1.000000 \mathrm{ul} & & \\ \text { Sample Amount } & : 1.0000 & \text { Area Reject } & : 0.000000 \\ \text { Data Acquisition Time } & : 6 / 11 / 20135: 42: 36 \mathrm{PM} & \text { Dilution Factor }: 1.00\end{array}$

Raw Data File : C:IHPLC DatalrobinlEthanol_June 10-2013IEthanol011.raw

Result File : c:Ihplc datalrobinlethanol_june 10-2013lethanol011.rst [Editing in Progress]

Inst Method : C:IHPLC DatalrobinlMethod Robin 87H from C:IHPLC DatalrobinlEthanol_June 10-2013।Ethanol011.raw

Proc Method : C:IHPLC DatalrobinlMethod Robin 87H from c:Ihplc datalrobinlethanol_june 10-2013lethanol011.rst [Editing in Progress] Calib Method : C:IHPLC DatalrobinlMethod Robin 87H from c:Ihplc datalrobinlethanol_june 10-2013lethanol011.rst [Editing in Progress]

Report Format File: C:IHPLC DatalrobinlMethod Robin 87H.rpt

Sequence File : C:IHPLC DatalrobinIValera Sequence 87H-Etanol_June 10_2013.seq

\begin{tabular}{|c|c|c|c|c|c|c|c|}
\hline $\begin{array}{l}\text { Peak } \\
\quad \#\end{array}$ & $\begin{array}{l}\text { Time } \\
\text { [min] }\end{array}$ & $\begin{array}{l}\text { Area } \\
{[\mu \mathrm{V} \cdot \mathrm{s}]}\end{array}$ & $\begin{array}{l}\text { Height } \\
{[\mu \mathrm{V}]}\end{array}$ & $\begin{array}{c}\text { Area } \\
{[\%]}\end{array}$ & $\begin{array}{c}\text { Norm. Area } \\
{[\%]}\end{array}$ & $B L$ & $\begin{array}{c}\text { Area/Height } \\
\text { [s] }\end{array}$ \\
\hline - & 0.001 & 0.00 & 0.00 & 0.00 & 0.00 & & \\
\hline 1 & 0.277 & 1279.20 & 111.38 & 0.00 & 0.00 & BB & 11.4850 \\
\hline 2 & 0.595 & 125.00 & 33.53 & 0.00 & 0.00 & BB & 3.7278 \\
\hline 3 & 1.085 & 177.20 & 39.22 & 0.00 & 0.00 & BB & 4.5184 \\
\hline 4 & 1.555 & 212.90 & 38.52 & 0.00 & 0.00 & BV & 5.5267 \\
\hline 5 & 1.719 & 378.99 & 60.64 & 0.00 & 0.00 & VV & 6.2501 \\
\hline 6 & 1.876 & 281.10 & 59.66 & 0.00 & 0.00 & VB & 4.7115 \\
\hline 7 & 2.733 & 84.00 & 51.26 & $7 e-05$ & $7 e-05$ & BB & 1.6387 \\
\hline 8 & 2.990 & 321.00 & 84.53 & 0.00 & 0.00 & BB & 3.7974 \\
\hline 9 & 3.319 & 286.00 & 46.83 & 0.00 & 0.00 & BB & 6.1077 \\
\hline 10 & 3.502 & 253.20 & 81.27 & 0.00 & 0.00 & BB & 3.1155 \\
\hline 11 & 4.448 & 190.80 & 49.03 & 0.00 & 0.00 & BB & 3.8918 \\
\hline 12 & 4.761 & 58.40 & 28.41 & $5 e-05$ & $5 e-05$ & BB & 2.0557 \\
\hline 13 & 5.080 & 218.60 & 78.00 & 0.00 & 0.00 & BB & 2.8024 \\
\hline 14 & 5.729 & 225.00 & 53.72 & 0.00 & 0.00 & BB & 4.1881 \\
\hline 15 & 5.874 & 168.91 & 61.74 & 0.00 & 0.00 & BV & 2.7359 \\
\hline 16 & 6.255 & 39932.78 & 5272.36 & 0.03 & 0.03 & VV & 7.5740 \\
\hline 17 & 6.587 & 5938679.47 & 243956.29 & 4.97 & 4.97 & VV & 24.3432 \\
\hline 18 & 7.294 & 3415176.78 & 102848.45 & 2.86 & 2.86 & VV & 33.2 \\
\hline 19 & 8.193 & 970817.25 & 63016.24 & 0.81 & 0.81 & VV & 15.4 \\
\hline 20 & 8.599 & 15232968.24 & 650597.16 & 12.75 & 12.75 & VV & 23.4 \\
\hline 21 & 9.293 & 57273847.59 & 993678.72 & 47.94 & 47.94 & VE & 57.6382 \\
\hline 22 & 11.678 & 625340.40 & 9031.73 & 0.52 & 0.52 & $E V$ & 69.2381 \\
\hline 23 & 13.394 & 1813674.18 & 54228.32 & 1.52 & 1.52 & VB & 33.4451 \\
\hline 24 & 14.714 & 152.80 & 95.09 & 0.00 & 0.00 & BB & 1.6069 \\
\hline 25 & 14.780 & 196.73 & 65.54 & 0.00 & 0.00 & BV & 3.0016 \\
\hline 26 & 14.882 & 155.78 & 79.94 & 0.00 & 0.00 & VV & 1.9487 \\
\hline 27 & 15.364 & 633974.69 & 20336.52 & 0.53 & 0.53 & VB & 31.1742 \\
\hline 28 & 16.369 & 59.60 & 70.33 & $5 e-05$ & $5 e-05$ & BB & 0.8474 \\
\hline 29 & 16.408 & 93.60 & 58.75 & $8 e-05$ & $8 e-05$ & BB & 1.5931 \\
\hline 30 & 16.648 & 121.80 & 74.67 & 0.00 & 0.00 & BB & 1.6 \\
\hline 31 & 16.711 & 257.40 & 92.72 & 0.00 & 0.00 & BB & 2.7761 \\
\hline 32 & 16.802 & 56.40 & 38.57 & $5 e-05$ & $5 e-05$ & BB & 1.4621 \\
\hline 33 & 16.868 & 122.00 & 80.39 & 0.00 & 0.00 & BB & 1.5175 \\
\hline 34 & 17.135 & 109.77 & 88.44 & $9 e-05$ & $9 e-05$ & BV & 1.241 \\
\hline 35 & 17.203 & 266.23 & 79.23 & 0.00 & 0.00 & VB & 3.360 \\
\hline 36 & 17.281 & 113.80 & 77.95 & $1 e-04$ & $1 e-04$ & BB & 1.4598 \\
\hline 37 & 17.382 & 242.52 & 66.99 & 0.00 & 0.00 & BV & 3.6201 \\
\hline 38 & 17.460 & 100.08 & 63.59 & $8 e-05$ & $8 e-05$ & VB & 1.5740 \\
\hline 39 & 17.573 & 228.60 & 98.86 & 0.00 & 0.00 & BB & 2.3123 \\
\hline 40 & 17.692 & 151.60 & 51.19 & 0.00 & 0.00 & BB & 2.9617 \\
\hline 41 & 17.770 & 114.80 & 73.00 & $1 e-04$ & $1 e-04$ & BB & 1.5727 \\
\hline 42 & 17.889 & 82.80 & 76.26 & $7 e-05$ & $7 e-05$ & BB & 1.0858 \\
\hline 43 & 17.922 & 53.80 & 53.04 & $5 e-05$ & $5 e-05$ & BB & 1.0142 \\
\hline 44 & 17.998 & 72.70 & 91.45 & $6 e-05$ & $6 e-05$ & BV & 0.7950 \\
\hline 45 & 18.087 & 945.04 & 212.55 & 0.00 & 0.00 & VV & 17.446 \\
\hline
\end{tabular}


6/21/2013 12:53:00 PM Result: c:Ihplc datalrobinlethanol_june 10-2013lethanol011.rst

\begin{tabular}{|c|c|c|c|c|c|c|c|}
\hline $\begin{array}{l}\text { Peak } \\
\quad \#\end{array}$ & $\begin{array}{l}\text { Time } \\
\text { [min] }\end{array}$ & $\begin{array}{l}\text { Area } \\
{[\mu \mathrm{V} \cdot \mathrm{s}]}\end{array}$ & $\begin{array}{l}\text { Height } \\
{[\mu \mathrm{V}]}\end{array}$ & $\begin{array}{c}\text { Area } \\
{[\%]}\end{array}$ & $\begin{array}{c}\text { Norm. Area } \\
{[\%]}\end{array}$ & BL & $\begin{array}{c}\text { Area/Height } \\
{[\mathrm{s}]}\end{array}$ \\
\hline 46 & 18.143 & 904.81 & 324.20 & 0.00 & 0.00 & VV & 2.7909 \\
\hline 47 & 18.185 & 1059.79 & 312.83 & 0.00 & 0.00 & VV & 3.3877 \\
\hline 48 & 18.252 & 914.85 & 278.48 & 0.00 & 0.00 & VB & 3.2852 \\
\hline 49 & 18.533 & 330.00 & 93.06 & 0.00 & 0.00 & BB & 3.5460 \\
\hline 50 & 19.305 & 6465.80 & 180.23 & 0.01 & 0.01 & $\mathrm{BB}$ & 35.8758 \\
\hline 51 & 19.981 & 366.40 & 127.66 & 0.00 & 0.00 & BB & 2.8700 \\
\hline 52 & 20.232 & 642.94 & 133.30 & 0.00 & 0.00 & $\mathrm{BV}$ & 4.8231 \\
\hline 53 & 20.348 & 614.11 & 109.68 & 0.00 & 0.00 & VV & 5.5990 \\
\hline 54 & 20.377 & 84.03 & 86.18 & $7 e-05$ & $7 e-05$ & VV & 0.9751 \\
\hline 55 & 20.411 & 510.52 & 135.37 & 0.00 & 0.00 & VB & 3.7713 \\
\hline 56 & 20.669 & 660.75 & 186.68 & 0.00 & 0.00 & BV & 3.5394 \\
\hline 57 & 21.523 & 33503603.93 & 833111.27 & 28.04 & 28.04 & VB & 40.2150 \\
\hline 58 & 23.396 & 54.80 & 57.43 & $5 e-05$ & $5 e-05$ & BB & 0.9542 \\
\hline 59 & 23.488 & 432.39 & 111.32 & 0.00 & 0.00 & BV & 3.8842 \\
\hline \multirow[t]{2}{*}{60} & 23.559 & 1615.61 & 149.68 & 0.00 & 0.00 & VB & 10.7939 \\
\hline & & $1.19 e+08$ & $2.98 \mathrm{e}+06$ & 100.00 & 100.00 & & \\
\hline
\end{tabular}

Warning -- Signal level out-of-range in peak

Missing Component Report

Component Expected Retention (Calibration File)

standards

0.001 
Time of Injection: 6/11/2013 5:42:36 PM

Start Time : $0.00 \mathrm{~min} \quad$ End Time : $24.99 \mathrm{~min} \quad$ Low Point : $-44.72 \mathrm{mV}$

Scale Factor: $1.0 \quad$ Plot Offset: $-44.72 \mathrm{mV}$

Plot Scale: $1044.7 \mathrm{mV}$

High Point : $1000.00 \mathrm{mV}$

Response [mV]

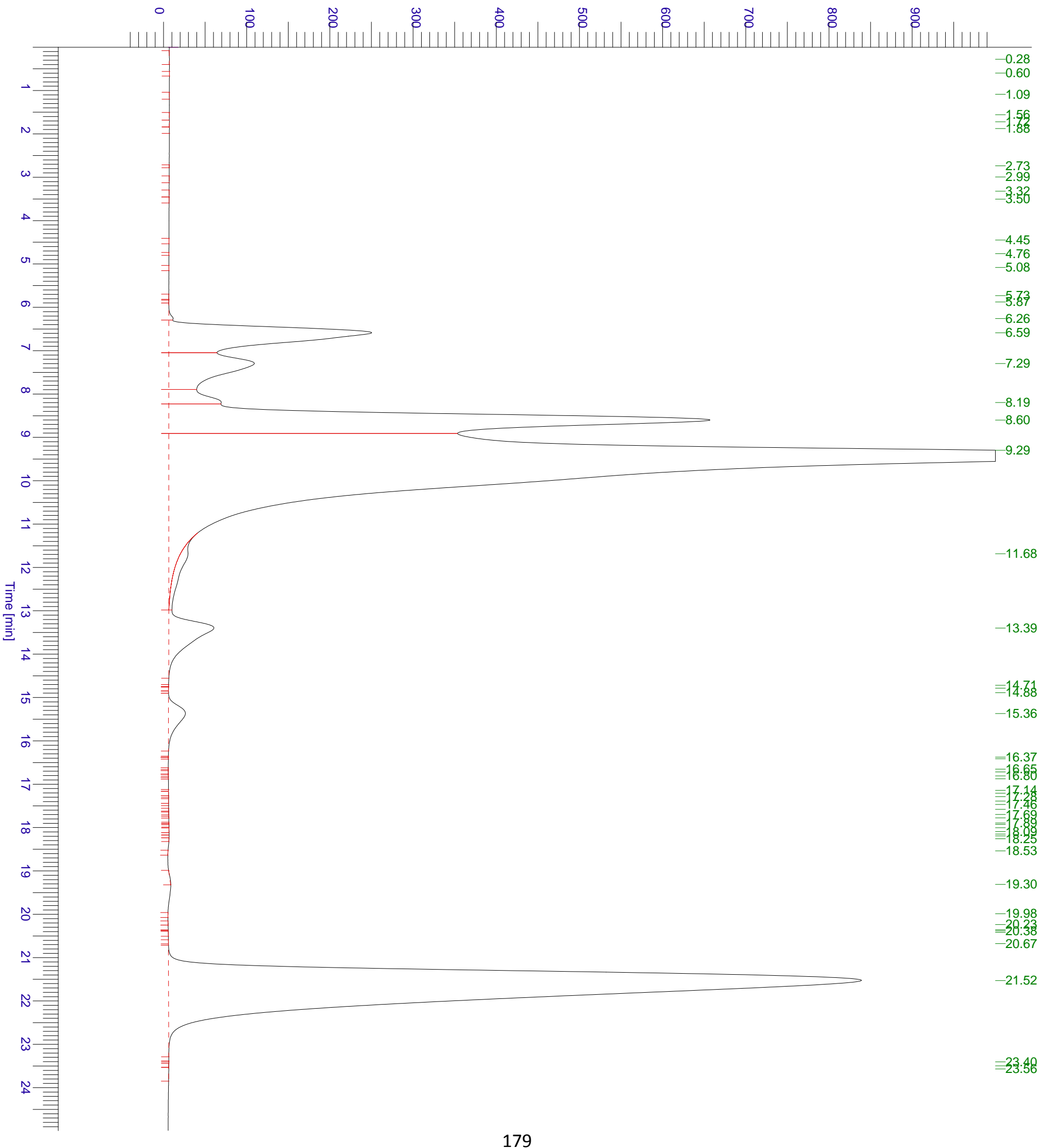




$\begin{array}{llll}\text { Software Version } & : 6.3 .2 .0646 & \text { Date } & : 6 / 21 / 2013 \text { 12:53:44 PM } \\ \text { Operator } & : \text { rLuong } & \text { Sample Name } & : \text { S5 } \\ \text { Sample Number } & : \text { O12 } & \text { Study } & : \text { Ethanol } \\ \text { AutoSampler } & : \text { SER200 } & \text { Rack/Vial } & : 1 / 12 \\ \text { Instrument Name } & : \text { HPLC } & \text { Channel } & : \text { A } \\ \text { Instrument Serial \# } & : \text { None } & \text { A/D mV Range }: 1000 \\ \text { Delay Time } & : 0.00 \mathrm{~min} & \text { End Time } & : 24.99 \mathrm{~min} \\ \text { Sampling Rate } & : 2.5000 \mathrm{pts} / \mathrm{s} & & \\ \text { Sample Volume } & : 1.000000 \mathrm{ul} & & \\ \text { Sample Amount } & : 1.0000 & \text { Area Reject } & : 0.000000 \\ \text { Data Acquisition Time } & : 6 / 11 / 2013 \mathrm{6:08:52} \mathrm{PM} & \text { Dilution Factor }: 1.00\end{array}$

Raw Data File : C:IHPLC DatalrobinlEthanol_June 10-2013IEthanol012.raw

Result File : c:Ihplc datalrobinlethanol_june 10-2013lethanol012.rst [Editing in Progress]

Inst Method : C:IHPLC DatalrobinlMethod Robin 87H from C:IHPLC DatalrobinlEthanol June 10-2013।Ethanol012.raw

Proc Method : C:IHPLC DatalrobinlMethod Robin 87H from c:Ihplc datalrobinlethanol_june 10-2013lethanol012.rst [Editing in Progress] Calib Method : C:IHPLC DatalrobinlMethod Robin 87H from c:Ihplc datalrobinlethanol_june 10-2013lethanol012.rst [Editing in Progress] Report Format File: C:IHPLC DatalrobinlMethod Robin 87H.rpt

Sequence File : C:IHPLC DatalrobinIValera Sequence 87H-Etanol_June 10_2013.seq

\begin{tabular}{|c|c|c|c|c|c|c|c|}
\hline $\begin{array}{c}\text { Peak } \\
\#\end{array}$ & $\begin{array}{l}\text { Time } \\
\text { [min] }\end{array}$ & $\begin{array}{l}\text { Area } \\
{[\mu \mathrm{V} \cdot \mathrm{s}]}\end{array}$ & $\begin{array}{l}\text { Height } \\
{[\mu \mathrm{V}]}\end{array}$ & $\begin{array}{c}\text { Area } \\
{[\%]}\end{array}$ & $\begin{array}{c}\text { Norm. Area } \\
{[\%]}\end{array}$ & $\mathrm{BL}$ & $\begin{array}{c}\text { Area/Height } \\
\text { [s] }\end{array}$ \\
\hline - & 0.001 & 0.00 & 0.00 & 0.00 & 0.00 & & \\
\hline 1 & 0.220 & 495.20 & 84.97 & 0.00 & 0.00 & BB & 5.8282 \\
\hline 2 & 0.302 & 38.80 & 37.84 & $4 e-05$ & $4 e-05$ & BB & 1.0254 \\
\hline 3 & 0.500 & 54.00 & 48.93 & $5 e-05$ & $5 e-05$ & BB & 1.1036 \\
\hline 4 & 0.683 & 100.40 & 49.74 & $1 e-04$ & $1 e-04$ & BB & 2.0184 \\
\hline 5 & 0.827 & 57.00 & 58.10 & $5 e-05$ & $5 e-05$ & BB & 0.9811 \\
\hline 6 & 0.975 & 126.00 & 107.08 & 0.00 & 0.00 & BB & 1.1767 \\
\hline 7 & 1.007 & 68.00 & 68.57 & $6 e-05$ & $6 e-05$ & BB & 0.9917 \\
\hline 8 & 1.281 & 243.60 & 82.57 & 0.00 & 0.00 & BB & 2.9501 \\
\hline 9 & 1.416 & 442.60 & 101.22 & 0.00 & 0.00 & BB & 4.3727 \\
\hline 10 & 1.544 & 223.60 & 88.76 & 0.00 & 0.00 & BB & 2.5191 \\
\hline 11 & 1.610 & 55.60 & 78.04 & $5 e-05$ & $5 e-05$ & BB & 0.7125 \\
\hline 12 & 1.646 & 653.40 & 104.37 & 0.00 & 0.00 & BB & 6.2602 \\
\hline 13 & 1.836 & 196.00 & 88.27 & 0.00 & 0.00 & BB & 2.2204 \\
\hline 14 & 1.905 & 204.81 & 129.94 & 0.00 & 0.00 & BV & 1.5762 \\
\hline 15 & 1.933 & 306.92 & 129.70 & 0.00 & 0.00 & VV & 2.3663 \\
\hline 16 & 1.992 & 89.47 & 77.96 & $8 e-05$ & $8 e-05$ & VB & 1.1477 \\
\hline 17 & 2.036 & 158.40 & 66.40 & 0.00 & 0.00 & BB & 2.3855 \\
\hline 18 & 2.120 & 96.40 & 97.39 & $9 e-05$ & $9 e-05$ & BB & 0.9898 \\
\hline 19 & 2.154 & 339.20 & 94.22 & 0.00 & 0.00 & BB & 3.6000 \\
\hline 20 & 2.446 & 493.43 & 140.07 & 0.00 & 0.00 & BV & 3.5228 \\
\hline 21 & 2.507 & 90.57 & 85.51 & $9 e-05$ & $9 e-05$ & VB & 1.0592 \\
\hline 22 & 2.549 & 169.00 & 101.31 & 0.00 & 0.00 & BB & 1.6682 \\
\hline 23 & 2.766 & 148.20 & 96.98 & 0.00 & 0.00 & BB & 1.5281 \\
\hline 24 & 2.827 & 463.60 & 100.71 & 0.00 & 0.00 & BB & 4.6034 \\
\hline 25 & 2.905 & 65.00 & 67.72 & $6 e-05$ & $6 e-05$ & BB & 0.9599 \\
\hline 26 & 3.028 & 72.60 & 79.26 & $7 e-05$ & $7 e-05$ & BB & 0.9160 \\
\hline 27 & 3.073 & 144.40 & 64.84 & 0.00 & 0.00 & BB & 2.2271 \\
\hline 28 & 3.158 & 64.00 & 64.56 & $6 e-05$ & $6 e-05$ & BB & 0.9913 \\
\hline 29 & 3.227 & 458.40 & 108.36 & 0.00 & 0.00 & BB & 4.2302 \\
\hline 30 & 3.354 & 58.00 & 59.07 & $5 e-05$ & $5 e-05$ & BB & 0.9819 \\
\hline 31 & 3.387 & 109.40 & 57.83 & 0.00 & 0.00 & BB & 1.8918 \\
\hline 32 & 3.571 & 258.80 & 88.90 & 0.00 & 0.00 & BB & 2.9113 \\
\hline 33 & 3.714 & 814.60 & 150.27 & 0.00 & 0.00 & BB & 5.4211 \\
\hline 34 & 3.842 & 328.00 & 108.07 & 0.00 & 0.00 & BB & 3.0351 \\
\hline 35 & 3.971 & 240.40 & 97.44 & 0.00 & 0.00 & BB & 2.4672 \\
\hline 36 & 4.120 & 40.40 & 35.79 & $4 e-05$ & $4 e-05$ & BB & 1.1289 \\
\hline 37 & 4.380 & 150.62 & 90.11 & 0.00 & 0.00 & BV & 1.6716 \\
\hline 38 & 4.415 & 253.58 & 83.10 & 0.00 & 0.00 & VB & 3.0516 \\
\hline 39 & 4.552 & 38.40 & 52.14 & $4 e-05$ & $4 e-05$ & BB & 0.7364 \\
\hline 40 & 4.713 & 218.20 & 71.67 & 0.00 & 0.00 & BB & 3.0443 \\
\hline 41 & 4.857 & 202.40 & 73.75 & 0.00 & 0.00 & BB & 2.7446 \\
\hline 42 & 5.018 & 436.00 & 82.95 & 0.00 & 0.00 & BB & 5.2560 \\
\hline 43 & 5.170 & 112.80 & 47.57 & 0.00 & 0.00 & BB & 2.3712 \\
\hline 44 & 5.490 & 506.40 & 98.89 & 0.00 & 0.00 & BB & 5.1207 \\
\hline 45 & 5.668 & 210.60 & 71.02 & 0.00 & 0.00 & $\mathrm{BB}$ & 189654 \\
\hline
\end{tabular}




\begin{tabular}{|c|c|c|c|c|c|c|c|}
\hline $\begin{array}{c}\text { Peak } \\
\#\end{array}$ & $\begin{array}{l}\text { Time } \\
\text { [min] }\end{array}$ & $\begin{array}{c}\text { Area } \\
{[\mu \mathrm{V} \cdot \mathrm{s}]}\end{array}$ & $\begin{array}{l}\text { Height } \\
{[\mu \mathrm{V}]}\end{array}$ & $\begin{array}{c}\text { Area } \\
{[\%]}\end{array}$ & $\begin{array}{c}\text { Norm. Area } \\
{[\%]}\end{array}$ & $B L$ & $\begin{array}{c}\text { Area/Height } \\
{[\mathrm{s}]}\end{array}$ \\
\hline 46 & 5.795 & 73.20 & 62.35 & $7 e-05$ & $7 e-05$ & BB & 1.1741 \\
\hline 47 & 6.260 & 39588.99 & 5262.68 & 0.04 & 0.04 & BV & 7.5226 \\
\hline 48 & 6.588 & 5460282.52 & 224549.54 & 5.17 & 5.17 & VV & 24.3166 \\
\hline 49 & 7.292 & 3088469.08 & 95122.43 & 2.92 & 2.92 & VV & 32.4684 \\
\hline 50 & 8.189 & 939755.31 & 58582.35 & 0.89 & 0.89 & VV & 16.0416 \\
\hline 51 & 8.600 & 12805995.97 & 543270.64 & 12.12 & 12.12 & VV & 23.5720 \\
\hline 52 & 9.405 & 46562236.85 & 812753.17 & 44.06 & 44.06 & VV & 57.2895 \\
\hline 53 & 11.590 & 38911.10 & 17505.51 & 0.04 & 0.04 & VV & 2.2228 \\
\hline 54 & 11.677 & 709439.53 & 17614.04 & 0.67 & 0.67 & VV & 40.2769 \\
\hline 55 & 13.406 & 4757173.64 & 138832.55 & 4.50 & 4.50 & VB & 34.2656 \\
\hline 56 & 14.749 & 291.60 & 104.86 & 0.00 & 0.00 & BB & 2.7808 \\
\hline 57 & 14.833 & 75.80 & 76.87 & $7 e-05$ & $7 e-05$ & BB & 0.9861 \\
\hline 58 & 15.370 & 1046583.00 & 33608.38 & 0.99 & 0.99 & BB & 31.1405 \\
\hline 59 & 16.905 & 153.78 & 62.83 & 0.00 & 0.00 & BV & 2.4477 \\
\hline 60 & 16.958 & 99.82 & 48.42 & $9 e-05$ & $9 e-05$ & VB & 2.0613 \\
\hline 61 & 17.213 & 329.20 & 67.37 & 0.00 & 0.00 & BB & 4.8865 \\
\hline 62 & 17.373 & 505.20 & 101.74 & 0.00 & 0.00 & $\mathrm{BB}$ & 4.9657 \\
\hline 63 & 17.687 & 536.14 & 67.90 & 0.00 & 0.00 & BV & 7.8958 \\
\hline 64 & 18.101 & 5653.23 & 433.31 & 0.01 & 0.01 & VV & 13.0467 \\
\hline 65 & 18.160 & 4316.43 & 462.05 & 0.00 & 0.00 & VB & 9.3418 \\
\hline 66 & 18.688 & 91.57 & 55.83 & $9 e-05$ & $9 e-05$ & BV & 1.6402 \\
\hline 67 & 19.302 & 101185.22 & 3075.21 & 0.10 & 0.10 & VB & 32.9035 \\
\hline 68 & 20.133 & 306.10 & 76.18 & 0.00 & 0.00 & BV & 4.0183 \\
\hline 69 & 21.534 & 30105518.70 & 754596.23 & 28.49 & 28.49 & VB & 39.8962 \\
\hline 70 & 24.492 & 72.80 & 59.04 & $7 e-05$ & $7 e-05$ & BB & 1.2330 \\
\hline 71 & 24.696 & 124.80 & 51.87 & 0.00 & 0.00 & BV & 2.4062 \\
\hline 72 & 24.752 & 107.40 & 93.43 & 0.00 & 0.00 & VB & 1.1495 \\
\hline \multirow[t]{2}{*}{73} & 24.814 & 357.20 & 83.17 & 0.00 & 0.00 & BB & 4.2949 \\
\hline & & & $.11 E+U 0$ & & & & \\
\hline
\end{tabular}

Missing Component Report

Component Expected Retention (Calibration File) 
Time of Injection: 6/11/2013 6:08:52 PM

Start Time : $0.00 \mathrm{~min} \quad$ End Time : $24.99 \mathrm{~min}$

Low Point : $-34.59 \mathrm{mV}$

High Point : $819.95 \mathrm{mV}$

Scale Factor: $1.0 \quad$ Plot Offset: $-34.59 \mathrm{mV}$

Plot Scale: $854.5 \mathrm{mV}$

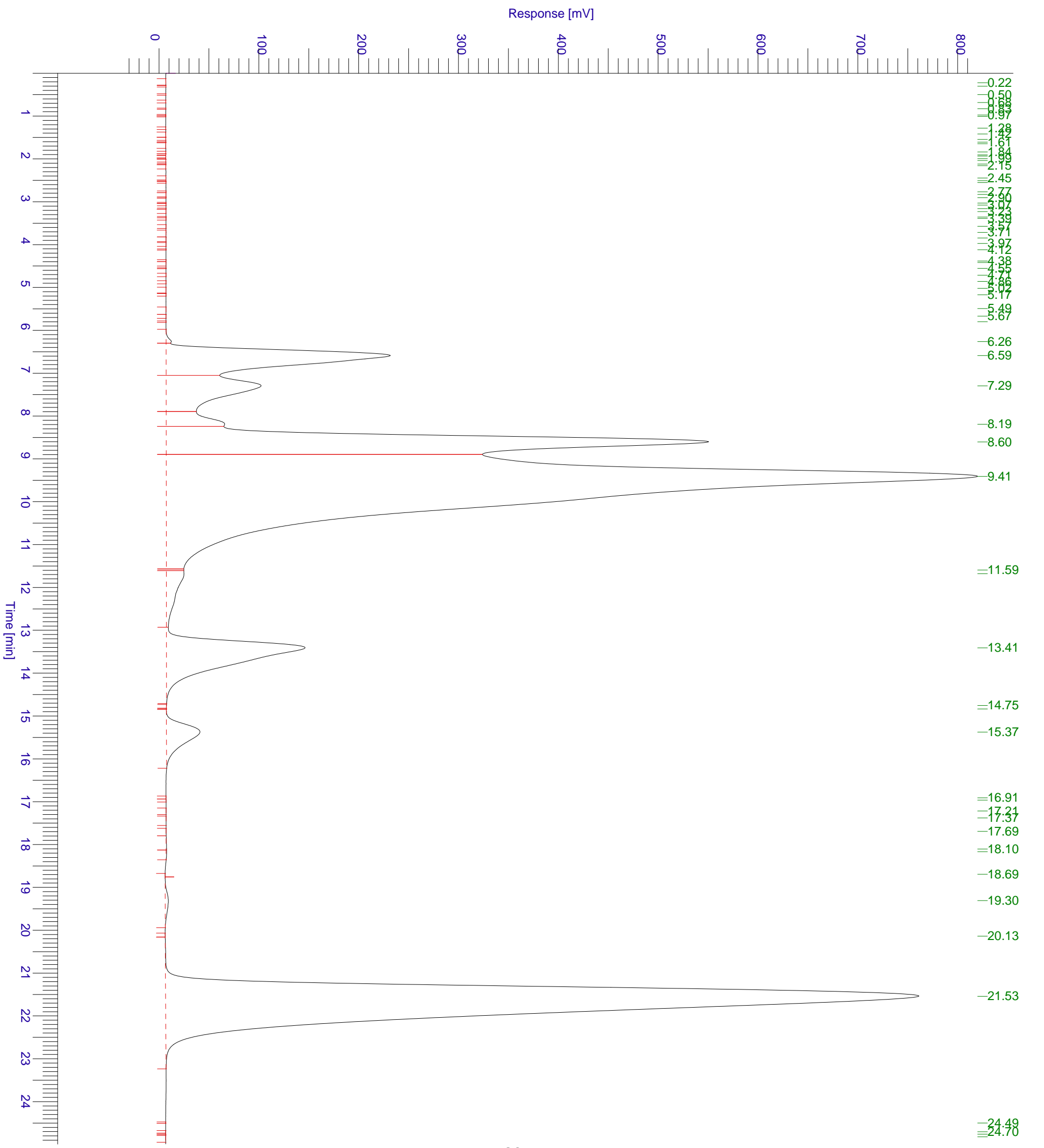




$\begin{array}{llll}\text { Software Version } & : 6.3 .2 .0646 & \text { Date } & : 6 / 21 / 201312: 54: 42 \text { PM } \\ \text { Operator } & : \text { rLuong } & \text { Sample Name } & : \text { S6 } \\ \text { Sample Number } & : 013 & \text { Study } & : \text { Ethanol } \\ \text { AutoSampler } & : \text { SER200 } & \text { Rack/Vial } & : 1 / 13 \\ \text { Instrument Name } & : \text { HPLC } & \text { Channel } & : \text { A } \\ \text { Instrument Serial \# } & : \text { None } & \text { A/D mV Range }: 1000 \\ \text { Delay Time } & : 0.00 \mathrm{~min} & \text { End Time } & : 24.99 \mathrm{~min} \\ \text { Sampling Rate } & : 2.5000 \mathrm{pts} / \mathrm{s} & & \\ \text { Sample Volume } & : 1.000000 \mathrm{ul} & & \\ \text { Sample Amount } & : 1.0000 & \text { Area Reject } & : 0.000000 \\ \text { Data Acquisition Time } & : 6 / 11 / 2013 \mathrm{6:35:09} \mathrm{PM} & \text { Dilution Factor }: 1.00\end{array}$

Raw Data File : C:IHPLC DatalrobinlEthanol_June 10-2013IEthanol013.raw

Result File : c:Ihplc datalrobinlethanol_june 10-2013lethanol013.rst [Editing in Progress]

Inst Method : C:IHPLC DatalrobinlMethod Robin 87H from C:IHPLC DatalrobinlEthanol June 10-2013।Ethanol013.raw

Proc Method : C:IHPLC DatalrobinlMethod Robin 87H from c:Ihplc datalrobinlethanol_june 10-2013lethanol013.rst [Editing in Progress] Calib Method : C:IHPLC DatalrobinlMethod Robin 87H from c:Ihplc datalrobinlethanol_june 10-2013lethanol013.rst [Editing in Progress]

Report Format File: C:IHPLC DatalrobinlMethod Robin 87H.rpt

Sequence File : C:IHPLC DatalrobinIValera Sequence 87H-Etanol_June 10_2013.seq

\begin{tabular}{|c|c|c|c|c|c|c|c|}
\hline $\begin{array}{c}\text { Peak } \\
\#\end{array}$ & $\begin{array}{l}\text { Time } \\
\text { [min] }\end{array}$ & $\begin{array}{l}\text { Area } \\
{[\mu \mathrm{V} \cdot \mathrm{s}]}\end{array}$ & $\begin{array}{l}\text { Height } \\
{[\mu \mathrm{V}]}\end{array}$ & $\begin{array}{c}\text { Area } \\
{[\%]}\end{array}$ & $\begin{array}{c}\text { Norm. Area } \\
{[\%]}\end{array}$ & $B L$ & $\begin{array}{c}\text { Area/Height } \\
\text { [s] }\end{array}$ \\
\hline - & 0.001 & 0.00 & 0.00 & 0.00 & 0.00 & & \\
\hline 1 & 0.103 & 177.20 & 86.26 & 0.00 & 0.00 & BB & 2.0543 \\
\hline 2 & 0.192 & 349.58 & 91.76 & 0.00 & 0.00 & BV & 3.8095 \\
\hline 3 & 0.270 & 189.22 & 48.21 & 0.00 & 0.00 & VB & 3.9253 \\
\hline 4 & 0.325 & 60.00 & 47.00 & $6 e-05$ & $6 e-05$ & BB & 1.2767 \\
\hline 5 & 0.435 & 286.00 & 76.58 & 0.00 & 0.00 & BB & 3.7345 \\
\hline 6 & 0.845 & 546.80 & 94.48 & 0.00 & 0.00 & BB & 5.7874 \\
\hline 7 & 1.178 & 28.80 & 32.64 & $3 e-05$ & $3 e-05$ & BB & 0.8823 \\
\hline 8 & 1.535 & 176.40 & 68.50 & 0.00 & 0.00 & BB & 2.5752 \\
\hline 9 & 1.709 & 114.80 & 75.16 & 0.00 & 0.00 & BB & 1.5275 \\
\hline 10 & 1.981 & 199.60 & 56.26 & 0.00 & 0.00 & BB & 3.5478 \\
\hline 11 & 2.185 & 170.20 & 58.35 & 0.00 & 0.00 & BB & 2.9170 \\
\hline 12 & 2.461 & 64.40 & 51.13 & $6 e-05$ & $6 e-05$ & BB & 1.2596 \\
\hline 13 & 2.659 & 73.20 & 44.61 & $7 e-05$ & $7 e-05$ & BB & 1.6409 \\
\hline 14 & 2.985 & 66.40 & 57.93 & $6 e-05$ & $6 e-05$ & BB & 1.1461 \\
\hline 15 & 3.236 & 119.60 & 69.06 & 0.00 & 0.00 & BB & 1.7319 \\
\hline 16 & 3.793 & 64.80 & 47.18 & $6 e-05$ & $6 e-05$ & BB & 1.3733 \\
\hline 17 & 4.048 & 125.00 & 64.13 & 0.00 & 0.00 & BB & 1.9491 \\
\hline 18 & 4.112 & 248.60 & 97.81 & 0.00 & 0.00 & BB & 2.5417 \\
\hline 19 & 4.278 & 131.20 & 62.53 & 0.00 & 0.00 & BB & 2.0984 \\
\hline 20 & 4.434 & 250.00 & 66.81 & 0.00 & 0.00 & BB & 3.7421 \\
\hline 21 & 4.799 & 73.66 & 79.26 & $7 e-05$ & $7 e-05$ & BV & 0.9293 \\
\hline 22 & 4.827 & 109.34 & 78.07 & 0.00 & 0.00 & VB & 1.4006 \\
\hline 23 & 5.083 & 251.60 & 100.07 & 0.00 & 0.00 & BB & 2.5142 \\
\hline 24 & 5.617 & 158.80 & 65.72 & 0.00 & 0.00 & BB & 2.4163 \\
\hline 25 & 5.903 & 155.20 & 65.86 & 0.00 & 0.00 & BB & 2.3566 \\
\hline 26 & 6.260 & 33235.55 & 4846.46 & 0.03 & 0.03 & BV & 6.8577 \\
\hline 27 & 6.595 & 5852781.47 & 238206.53 & 5.48 & 5.48 & VV & 24.5702 \\
\hline 28 & 7.294 & 3087462.57 & 98766.66 & 2.89 & 2.89 & VV & 31.2602 \\
\hline 29 & 8.183 & 908835.28 & 56885.61 & 0.85 & 0.85 & VV & 15.9765 \\
\hline 30 & 8.601 & 10426222.13 & 443359.96 & 9.76 & 9.76 & VV & 23.5164 \\
\hline 31 & 9.437 & 40309021.43 & 562440.36 & 37.74 & 37.74 & VV & 71.6681 \\
\hline 32 & 11.682 & 597.97 & 102.21 & 0.00 & 0.00 & VB & 5.8505 \\
\hline 33 & 13.412 & 8713618.80 & 260862.36 & 8.16 & 8.16 & BB & 33.4031 \\
\hline 34 & 15.374 & 2041794.80 & 64568.30 & 1.91 & 1.91 & $\mathrm{BB}$ & 31.6222 \\
\hline 35 & 16.718 & 100.60 & 53.59 & $9 \mathrm{e}-05$ & $9 e-05$ & BB & 1.8773 \\
\hline 36 & 16.791 & 93.60 & 37.28 & $9 e-05$ & $9 e-05$ & BB & 2.5110 \\
\hline 37 & 16.971 & 320.25 & 85.68 & 0.00 & 0.00 & BV & 3.7378 \\
\hline 38 & 17.163 & 456.15 & 49.84 & 0.00 & 0.00 & VB & 9.1521 \\
\hline 39 & 17.527 & 54.20 & 54.39 & $5 e-05$ & $5 e-05$ & BB & 0.9965 \\
\hline 40 & 18.089 & 1374.75 & 103.35 & 0.00 & 0.00 & BV & 13.3015 \\
\hline 41 & 18.141 & 224.05 & 90.82 & 0.00 & 0.00 & VB & 2.4669 \\
\hline 42 & 18.736 & 339.46 & 86.19 & 0.00 & 0.00 & BV & 3.9385 \\
\hline 43 & 19.324 & 110125.34 & 3379.56 & 0.10 & 0.10 & VB & 32.5857 \\
\hline 44 & 21.520 & 35321525.60 & 874316.07 & 33.07 & 33.07 & $\mathrm{BB}$ & 40.3990 \\
\hline 45 & 23.579 & 85.80 & 89.36 & $8 e-05$ & $8 e-05$ & $\mathrm{BB}$ & 18.9602 \\
\hline
\end{tabular}


6/21/2013 12:54:42 PM Result: c:Ihplc datalrobinlethanol_june 10-2013lethanol013.rst

\begin{tabular}{|c|c|c|c|c|c|c|c|}
\hline $\begin{array}{l}\text { Peak } \\
\quad \#\end{array}$ & $\begin{array}{l}\text { Time } \\
\text { [min] }\end{array}$ & $\begin{array}{l}\text { Area } \\
{[\mu \mathrm{V} \cdot \mathrm{s}]}\end{array}$ & $\begin{array}{l}\text { Height } \\
{[\mu \mathrm{V}]}\end{array}$ & $\begin{array}{c}\text { Area } \\
{[\%]}\end{array}$ & $\begin{array}{c}\text { Norm. Area } \\
{[\%]}\end{array}$ & $\mathrm{BL}$ & $\begin{array}{c}\text { Area/Height } \\
{[\mathrm{s}]}\end{array}$ \\
\hline 46 & 23.616 & 313.20 & 119.57 & 0.00 & 0.00 & BB & 2.6194 \\
\hline 47 & 23.763 & 320.40 & 83.57 & 0.00 & 0.00 & BB & 3.8338 \\
\hline 48 & 24.313 & 553.00 & 58.58 & 0.00 & 0.00 & BB & 9.4403 \\
\hline 49 & 24.516 & 426.00 & 117.47 & 0.00 & 0.00 & BB & 3.6265 \\
\hline 50 & 24.606 & 60.80 & 53.65 & $6 e-05$ & $6 e-05$ & BB & 1.1333 \\
\hline \multirow[t]{2}{*}{51} & 24.670 & 200.00 & 52.56 & 0.00 & 0.00 & $\mathrm{BB}$ & 3.8050 \\
\hline & & $1.07 e+08$ & $2.61 e+06$ & 100.00 & 100.00 & & \\
\hline
\end{tabular}

Missing Component Report

Component Expected Retention (Calibration File)

0.001 
Time of Injection: 6/11/2013 6:35:09 PM

Start Time : $0.00 \mathrm{~min} \quad$ End Time $: 24.99 \mathrm{~min}$

Low Point : $-38.41 \mathrm{mV}$

High Point : $880.44 \mathrm{mV}$

Scale Factor: 1.0

Plot Offset: - $38.41 \mathrm{mV}$

Plot Scale: $918.8 \mathrm{mV}$

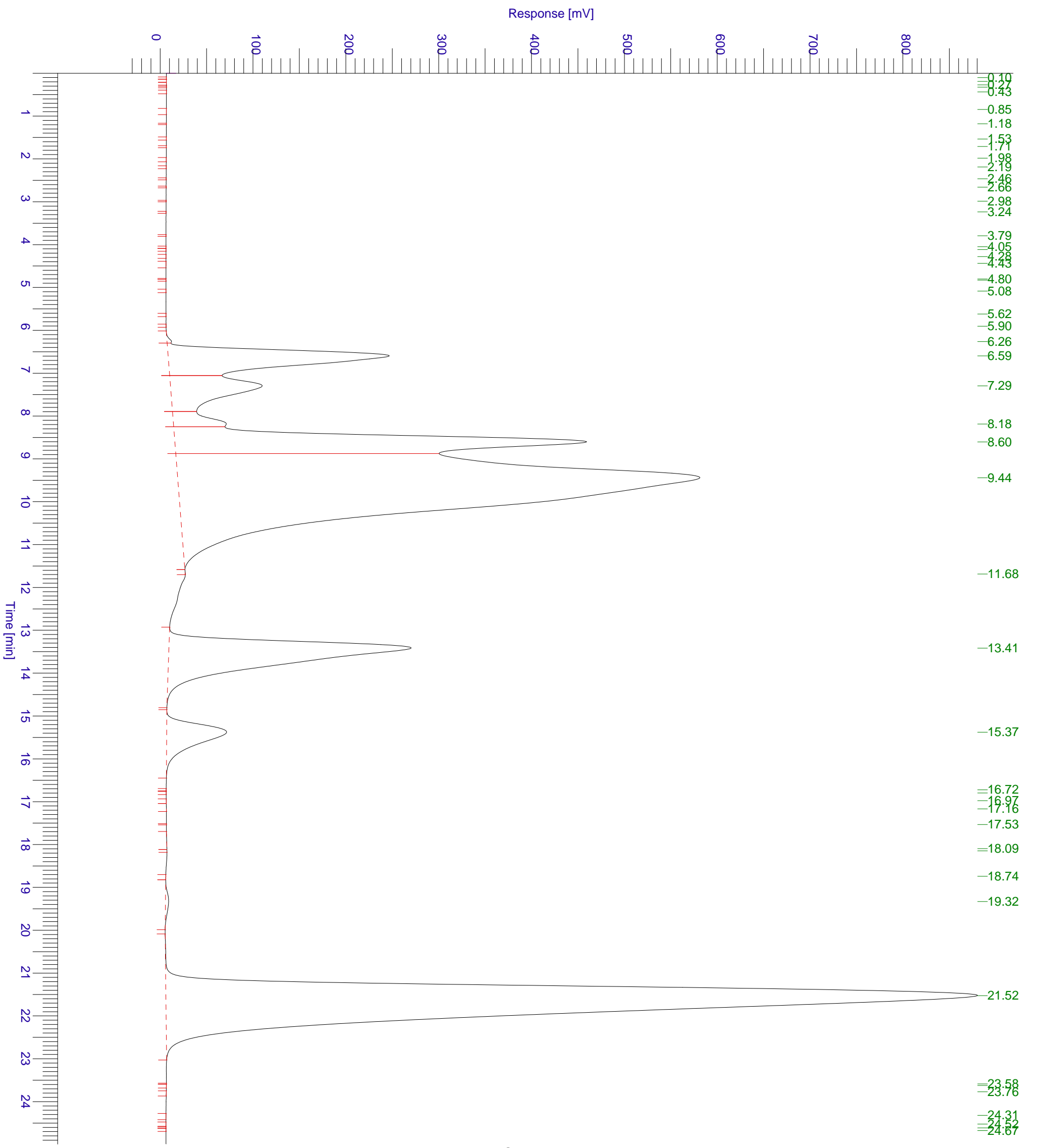




$\begin{array}{llll}\text { Software Version } & : 6.3 .2 .0646 & \text { Date } & : 6 / 21 / 2013 \text { 12:55:33 PM } \\ \text { Operator } & : \text { rLuong } & \text { Sample Name } & : \text { S7 } \\ \text { Sample Number } & : \text { O14 } & \text { Study } & : \text { Ethanol } \\ \text { AutoSampler } & : \text { SER200 } & \text { Rack/Vial } & : 1 / 14 \\ \text { Instrument Name } & : \text { HPLC } & \text { Channel } & : \text { A } \\ \text { Instrument Serial \# } & : \text { None } & \text { A/D mV Range }: 1000 \\ \text { Delay Time } & : 0.00 \mathrm{~min} & \text { End Time } & : 24.99 \mathrm{~min} \\ \text { Sampling Rate } & : 2.5000 \mathrm{pts} / \mathrm{s} & & \\ \text { Sample Volume } & : 1.000000 \mathrm{ul} & & \\ \text { Sample Amount } & : 1.0000 & \text { Area Reject } & : 0.000000 \\ \text { Data Acquisition Time } & : 6 / 11 / 2013 \text { 7:01:25 PM } & \text { Dilution Factor }: 1.00\end{array}$

Raw Data File : C:IHPLC DatalrobinlEthanol_June 10-2013IEthanol014.raw

Result File : c:Ihplc datalrobinlethanol_june 10-2013lethanol014.rst [Editing in Progress]

Inst Method : C:IHPLC DatalrobinlMethod Robin 87H from C:IHPLC DatalrobinlEthanol_June 10-2013।Ethanol014.raw

Proc Method : C:IHPLC DatalrobinlMethod Robin 87H from c:Ihplc datalrobinlethanol_june 10-2013lethanol014.rst [Editing in Progress] Calib Method : C:IHPLC DatalrobinlMethod Robin 87H from c:Ihplc datalrobinlethanol_june 10-2013lethanol014.rst [Editing in Progress]

Report Format File: C:IHPLC DatalrobinlMethod Robin 87H.rpt

Sequence File : C:IHPLC DatalrobinIValera Sequence 87H-Etanol_June 10_2013.seq

\begin{tabular}{|c|c|c|c|c|c|c|c|}
\hline $\begin{array}{c}\text { Peak } \\
\#\end{array}$ & $\begin{array}{l}\text { Time } \\
\text { [min] }\end{array}$ & $\begin{array}{l}\text { Area } \\
{[\mu \mathrm{V} \cdot \mathrm{s}]}\end{array}$ & $\begin{array}{c}\text { Height } \\
{[\mu \mathrm{V}]}\end{array}$ & $\begin{array}{c}\text { Area } \\
{[\%]}\end{array}$ & $\begin{array}{c}\text { Norm. Area } \\
{[\%]}\end{array}$ & $\mathrm{BL}$ & $\begin{array}{c}\text { Area/Height } \\
{[\mathrm{s}]}\end{array}$ \\
\hline - & 0.001 & 0.00 & 0.00 & 0.00 & 0.00 & & \\
\hline 1 & 0.182 & 701.94 & 102.73 & 0.00 & 0.00 & BV & 6.8329 \\
\hline 2 & 0.257 & 202.46 & 90.41 & 0.00 & 0.00 & VB & 2.2393 \\
\hline 3 & 0.429 & 248.80 & 57.23 & 0.00 & 0.00 & BB & 4.3472 \\
\hline 4 & 0.758 & 44.00 & 59.83 & $4 e-05$ & $4 e-05$ & BB & 0.7354 \\
\hline 5 & 0.819 & 57.20 & 44.50 & $5 e-05$ & $5 e-05$ & BB & 1.2853 \\
\hline 6 & 1.458 & 332.27 & 73.85 & 0.00 & 0.00 & BV & 4.4991 \\
\hline 7 & 1.550 & 172.13 & 58.53 & 0.00 & 0.00 & VB & 2.9410 \\
\hline 8 & 1.628 & 291.40 & 95.88 & 0.00 & 0.00 & BB & 3.0392 \\
\hline 9 & 1.817 & 342.60 & 85.27 & 0.00 & 0.00 & BB & 4.0179 \\
\hline 10 & 2.010 & 283.20 & 50.20 & 0.00 & 0.00 & BB & 5.6411 \\
\hline 11 & 2.436 & 434.80 & 126.67 & 0.00 & 0.00 & BB & 3.4326 \\
\hline 12 & 2.581 & 233.80 & 61.13 & 0.00 & 0.00 & BB & 3.8248 \\
\hline 13 & 2.760 & 498.80 & 76.80 & 0.00 & 0.00 & BB & 6.4946 \\
\hline 14 & 2.895 & 227.20 & 75.14 & 0.00 & 0.00 & BB & 3.0236 \\
\hline 15 & 3.226 & 130.80 & 57.57 & 0.00 & 0.00 & BB & 2.2720 \\
\hline 16 & 3.413 & 115.20 & 51.21 & 0.00 & 0.00 & BB & 2.2497 \\
\hline 17 & 3.554 & 394.80 & 78.98 & 0.00 & 0.00 & $\mathrm{BB}$ & 4.9989 \\
\hline 18 & 4.035 & 207.60 & 98.84 & 0.00 & 0.00 & BB & 2.1004 \\
\hline 19 & 4.707 & 125.80 & 71.18 & 0.00 & 0.00 & BB & 1.7673 \\
\hline 20 & 5.020 & 251.60 & 72.92 & 0.00 & 0.00 & $\mathrm{BB}$ & 3.4505 \\
\hline 21 & 5.343 & 335.60 & 94.22 & 0.00 & 0.00 & $\mathrm{BB}$ & 3.5620 \\
\hline 22 & 5.627 & 170.77 & 66.82 & 0.00 & 0.00 & BV & 2.5557 \\
\hline 23 & 5.689 & 230.13 & 70.59 & 0.00 & 0.00 & VV & 3.2599 \\
\hline 24 & 5.807 & 209.32 & 67.74 & 0.00 & 0.00 & VV & 3.0901 \\
\hline 25 & 6.570 & 3043072.04 & 108971.37 & 2.66 & 2.66 & VV & 27.9254 \\
\hline 26 & 7.306 & 3391274.76 & 88556.87 & 2.97 & 2.97 & VV & 38.2949 \\
\hline 27 & 8.610 & 17120747.91 & 694553.20 & 14.98 & 14.98 & VV & 24.6500 \\
\hline 28 & 9.273 & 57860520.07 & 953423.30 & 50.62 & 50.62 & VE & 60.6871 \\
\hline 29 & 11.670 & 225677.20 & 3600.14 & 0.20 & 0.20 & EV & 62.6856 \\
\hline 30 & 13.400 & 142700.21 & 3659.55 & 0.12 & 0.12 & VB & 38.9940 \\
\hline 31 & 15.345 & 43749.60 & 1519.06 & 0.04 & 0.04 & BB & 28.8004 \\
\hline 32 & 16.221 & 126.00 & 51.80 & 0.00 & 0.00 & $\mathrm{BB}$ & 2.4326 \\
\hline 33 & 16.382 & 95.80 & 51.42 & $8 e-05$ & $8 e-05$ & $\mathrm{BB}$ & 1.8629 \\
\hline 34 & 16.709 & 227.60 & 73.43 & 0.00 & 0.00 & $\mathrm{BB}$ & 3.0996 \\
\hline 35 & 16.879 & 370.42 & 71.87 & 0.00 & 0.00 & BV & 5.1543 \\
\hline 36 & 17.039 & 209.38 & 76.21 & 0.00 & 0.00 & VB & 2.7475 \\
\hline 37 & 17.387 & 91.20 & 29.39 & $8 e-05$ & $8 e-05$ & BB & 3.1034 \\
\hline 38 & 17.752 & 626.05 & 137.12 & 0.00 & 0.00 & BV & 4.5658 \\
\hline 39 & 17.935 & 2454.64 & 300.12 & 0.00 & 0.00 & VV & 8.1788 \\
\hline 40 & 18.322 & 30133.30 & 1102.69 & 0.03 & 0.03 & VB & 27.3270 \\
\hline 41 & 19.367 & 293.80 & 59.60 & 0.00 & 0.00 & BB & 4.9299 \\
\hline 42 & 19.598 & 73.60 & 47.65 & $6 e-05$ & $6 e-05$ & BB & 1.5447 \\
\hline 43 & 20.093 & 124.40 & 62.89 & 0.00 & 0.00 & BB & 1.9781 \\
\hline 44 & 20.254 & 114.80 & 51.14 & 0.00 & 0.00 & BB & 2.2450 \\
\hline 45 & 20.443 & 344.54 & 48.55 & 0.00 & 0.00 & BV & $1 \bar{\phi} .9960$ \\
\hline
\end{tabular}


6/21/2013 12:55:33 PM Result: c:Ihplc datalrobinlethanol june 10-2013lethanol014.rst

\begin{tabular}{|c|c|c|c|c|c|c|c|}
\hline $\begin{array}{c}\text { Peak } \\
\#\end{array}$ & $\begin{array}{l}\text { Time } \\
\text { [min] }\end{array}$ & $\begin{array}{l}\text { Area } \\
{[\mu \mathrm{V} \cdot \mathrm{s}]}\end{array}$ & $\begin{array}{l}\text { Height } \\
{[\mu \mathrm{V}]}\end{array}$ & $\begin{array}{c}\text { Area } \\
{[\%]}\end{array}$ & $\begin{array}{c}\text { Norm. Area } \\
{[\%]}\end{array}$ & $B L$ & $\begin{array}{c}\text { Area/Height } \\
{[\mathrm{s}]}\end{array}$ \\
\hline 46 & 21.530 & 32427737.46 & 809940.45 & 28.37 & 28.37 & VB & 40.0372 \\
\hline 47 & 23.200 & 279.60 & 103.63 & 0.00 & 0.00 & BB & 2.6980 \\
\hline 48 & 23.537 & 161.40 & 103.97 & 0.00 & 0.00 & BB & 1.5523 \\
\hline 49 & 23.635 & 172.40 & 73.85 & 0.00 & 0.00 & BB & 2.3343 \\
\hline 50 & 23.703 & 202.00 & 68.78 & 0.00 & 0.00 & BB & 2.9368 \\
\hline 51 & 23.772 & 211.60 & 64.49 & 0.00 & 0.00 & BB & 3.2812 \\
\hline 52 & 23.968 & 103.20 & 78.92 & $9 e-05$ & $9 e-05$ & BB & 1.3076 \\
\hline 53 & 24.027 & 127.20 & 63.29 & 0.00 & 0.00 & BB & 2.0099 \\
\hline 54 & 24.282 & 187.73 & 56.92 & 0.00 & 0.00 & BV & 3.2982 \\
\hline 55 & 24.333 & 56.27 & 56.09 & $5 e-05$ & $5 e-05$ & VB & 1.0032 \\
\hline \multirow[t]{2}{*}{56} & 24.473 & 163.40 & 79.32 & 0.00 & 0.00 & BB & 2.0599 \\
\hline & & $1.14 \mathrm{e}+0$ & $2.67 e+06$ & 100.00 & 100.00 & & \\
\hline
\end{tabular}

Warning -- Signal level out-of-range in peak

Missing Component Report

Component Expected Retention (Calibration File) 
Plot Scale: $1044.1 \mathrm{mV}$

Response [mV]

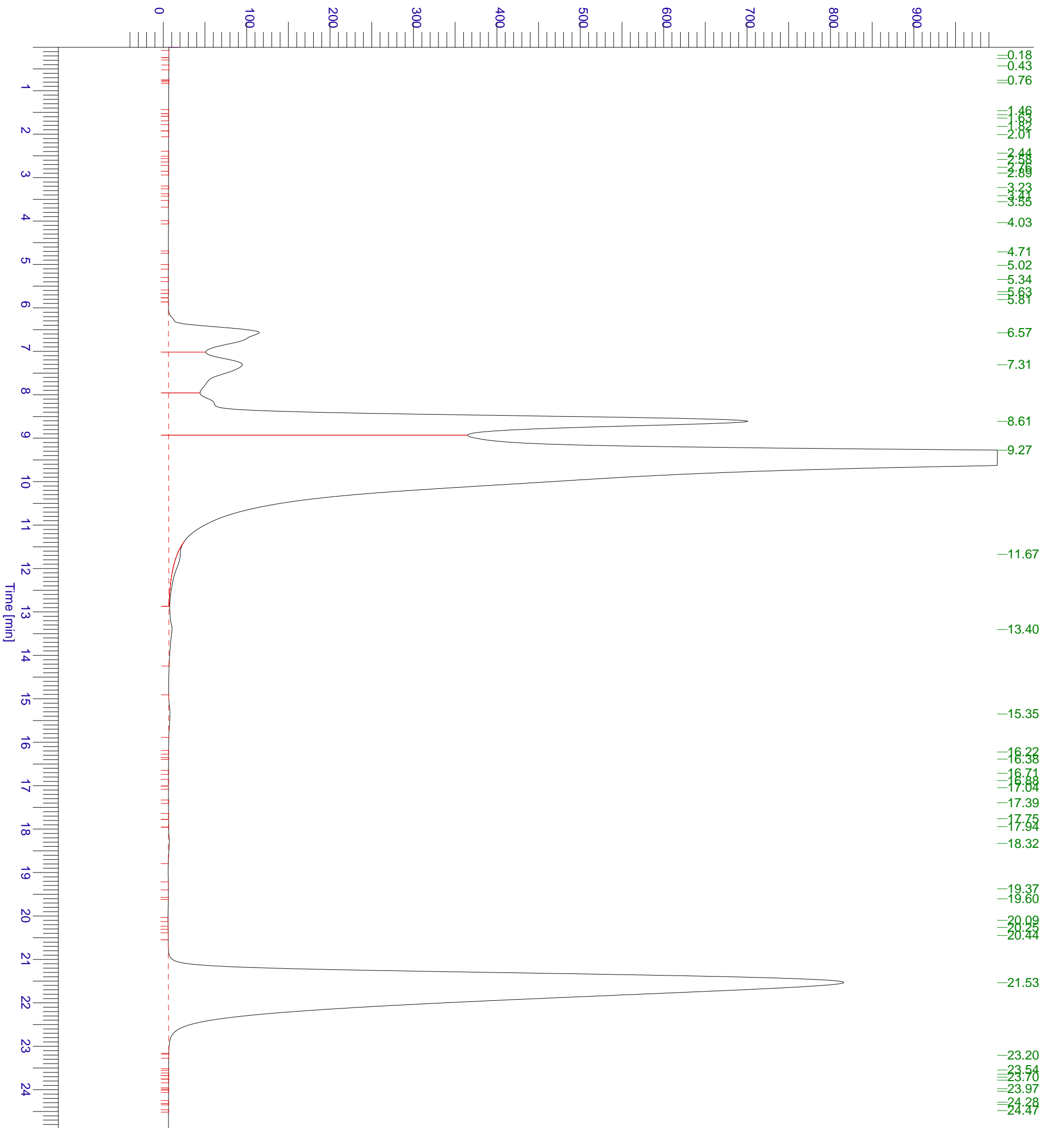




$\begin{array}{llll}\text { Software Version } & : 6.3 .2 .0646 & \text { Date } & : 6 / 21 / 20132: 16: 12 \text { PM } \\ \text { Operator } & : \text { rLuong } & \text { Sample Name } & : \text { S70 } \\ \text { Sample Number } & : \text { O77 } & \text { Study } & : \text { Ethanol } \\ \text { AutoSampler } & : \text { SER200 } & \text { Rack/Vial } & : 1 / 77 \\ \text { Instrument Name } & : \text { HPLC } & \text { Channel } & : \text { A } \\ \text { Instrument Serial \# } & : \text { None } & \text { A/D mV Range }: 1000 \\ \text { Delay Time } & : 0.00 \mathrm{~min} & \text { End Time } & : 24.99 \mathrm{~min} \\ \text { Sampling Rate } & : 2.5000 \mathrm{pts} / \mathrm{s} & & \\ \text { Sample Volume } & : 1.000000 \mathrm{ul} & & \\ \text { Sample Amount } & : 1.0000 & \text { Area Reject } & : 0.000000 \\ \text { Data Acquisition Time } & : 6 / 12 / 2013 \text { 10:38:29 PM } & \text { Dilution Factor }: 1.00\end{array}$

Raw Data File : C:IHPLC DatalrobinlEthanol_June 10-2013IEthanol077.raw

Result File : c:Ihplc datalrobinlethanol_june 10-2013lethanol077.rst [Editing in Progress]

Inst Method : C:IHPLC DatalrobinlMethod Robin 87H from C:IHPLC DatalrobinlEthanol June 10-2013।Ethanol077.raw

Proc Method : C:IHPLC DatalrobinlMethod Robin 87H from c:Ihplc datalrobinlethanol_june 10-2013lethanol077.rst [Editing in Progress] Calib Method : C:IHPLC DatalrobinlMethod Robin 87H from c:Ihplc datalrobinlethanol june 10-2013lethanol077.rst [Editing in Progress]

Report Format File: C:IHPLC DatalrobinlMethod Robin 87H.rpt

Sequence File : C:IHPLC DatalrobinIValera Sequence 87H-Etanol_June 10_2013.seq

\begin{tabular}{|c|c|c|c|c|c|c|c|}
\hline $\begin{array}{l}\text { Peak } \\
\quad \#\end{array}$ & $\begin{array}{l}\text { Time } \\
\text { [min] }\end{array}$ & $\begin{array}{l}\text { Area } \\
{[\mu \mathrm{V} \cdot \mathrm{s}]}\end{array}$ & $\begin{array}{l}\text { Height } \\
{[\mu \mathrm{V}]}\end{array}$ & $\begin{array}{c}\text { Area } \\
{[\%]}\end{array}$ & $\begin{array}{c}\text { Norm. Area } \\
{[\%]}\end{array}$ & BL & $\begin{array}{c}\text { Area/Heigh } \\
\text { [s] }\end{array}$ \\
\hline - & 0.001 & 0.00 & 0.00 & 0.00 & 0.00 & & \\
\hline 1 & 0.905 & 64556.80 & 4510.92 & 0.07 & 0.07 & BB & 14.3112 \\
\hline 2 & 1.133 & 129.60 & 85.60 & 0.00 & 0.00 & BB & 1.5141 \\
\hline 3 & 1.161 & 99.20 & 103.77 & 0.00 & 0.00 & BB & 0.9560 \\
\hline 4 & 1.198 & 743.40 & 271.10 & 0.00 & 0.00 & BB & 2.7421 \\
\hline 5 & 1.655 & 80.40 & 25.01 & $9 e-05$ & $9 e-05$ & BB & 3.2141 \\
\hline 6 & 2.208 & 309.26 & 49.53 & 0.00 & 0.00 & BV & 6.2436 \\
\hline 7 & 2.315 & 109.74 & 28.72 & 0.00 & 0.00 & VB & 3.8211 \\
\hline 8 & 3.134 & 702.00 & 36.70 & 0.00 & 0.00 & BB & 19.1298 \\
\hline 9 & 3.252 & 74.40 & 22.43 & $8 e-05$ & $8 e-05$ & BB & 3.3175 \\
\hline 10 & 3.429 & 77.80 & 26.86 & $9 e-05$ & $9 e-05$ & BB & 2.8969 \\
\hline 11 & 3.594 & 3140.82 & 201.05 & 0.00 & 0.00 & BV & 15.6224 \\
\hline 12 & 4.080 & 42154.04 & 2358.38 & 0.05 & 0.05 & VV & 17.8741 \\
\hline 13 & 4.227 & 26738.43 & 3011.27 & 0.03 & 0.03 & VV & 8.8794 \\
\hline 14 & 5.522 & 487942.38 & 8894.10 & 0.53 & 0.53 & VV & 54.8613 \\
\hline 15 & 6.600 & 3411935.02 & 134921.49 & 3.73 & 3.73 & VV & 25.2883 \\
\hline 16 & 6.968 & 4341005.67 & 159498.19 & 4.74 & 4.74 & VV & 27.2166 \\
\hline 17 & 7.684 & 8886628.65 & 325662.36 & 9.71 & 9.71 & VV & 27.2879 \\
\hline 18 & 8.686 & 17339924.75 & 677301.41 & 18.95 & 18.95 & VV & 25.6015 \\
\hline 19 & 9.354 & 24353455.84 & 484550.58 & 26.62 & 26.62 & VB & 50.2599 \\
\hline 20 & 11.926 & 1065996.40 & 25816.32 & 1.17 & 1.17 & BB & 41.2916 \\
\hline 21 & 12.741 & 509.97 & 118.04 & 0.00 & 0.00 & BV & 4.320 \\
\hline 22 & 13.524 & 339063.97 & 8639.88 & 0.37 & 0.37 & VV & 39.2441 \\
\hline 23 & 14.023 & 161965.77 & 5497.75 & 0.18 & 0.18 & VV & 29.4604 \\
\hline 24 & 14.829 & 82305.06 & 4481.02 & 0.09 & 0.09 & VV & 18.3675 \\
\hline 25 & 15.174 & 70844.77 & 6582.37 & 0.08 & 0.08 & VV & 10.7628 \\
\hline 26 & 15.376 & 340806.87 & 9337.87 & 0.37 & 0.37 & VB & 36.4973 \\
\hline 27 & 18.376 & 130818.00 & 4068.31 & 0.14 & 0.14 & BB & 32.1553 \\
\hline 28 & 19.562 & 100141.20 & 21427.21 & 0.11 & 0.11 & BB & 4.6736 \\
\hline 29 & 21.399 & 19917628.98 & 730074.73 & 21.77 & 21.77 & BV & 27.2816 \\
\hline 30 & 21.690 & 10316929.62 & 535417.64 & 11.28 & 11.28 & VB & 19.2689 \\
\hline 31 & 24.627 & 111.20 & 24.86 & 0.00 & 0.00 & BB & 4.4733 \\
\hline & & & $. .15 \mathrm{e}+00$ & 00.00 & 100.00 & & \\
\hline
\end{tabular}

Missing Component Report

Component Expected Retention (Calibration File)

standards

0.001 
Time of Injection: 6/12/2013 10:38:29 PM

Scale Factor: $1.0 \quad$ Plot Offset: $-34.71 \mathrm{mV}$

Low Point : $-34.71 \mathrm{mV}$

High Point : $744.99 \mathrm{mV}$

Plot Scale: $779.7 \mathrm{mV}$

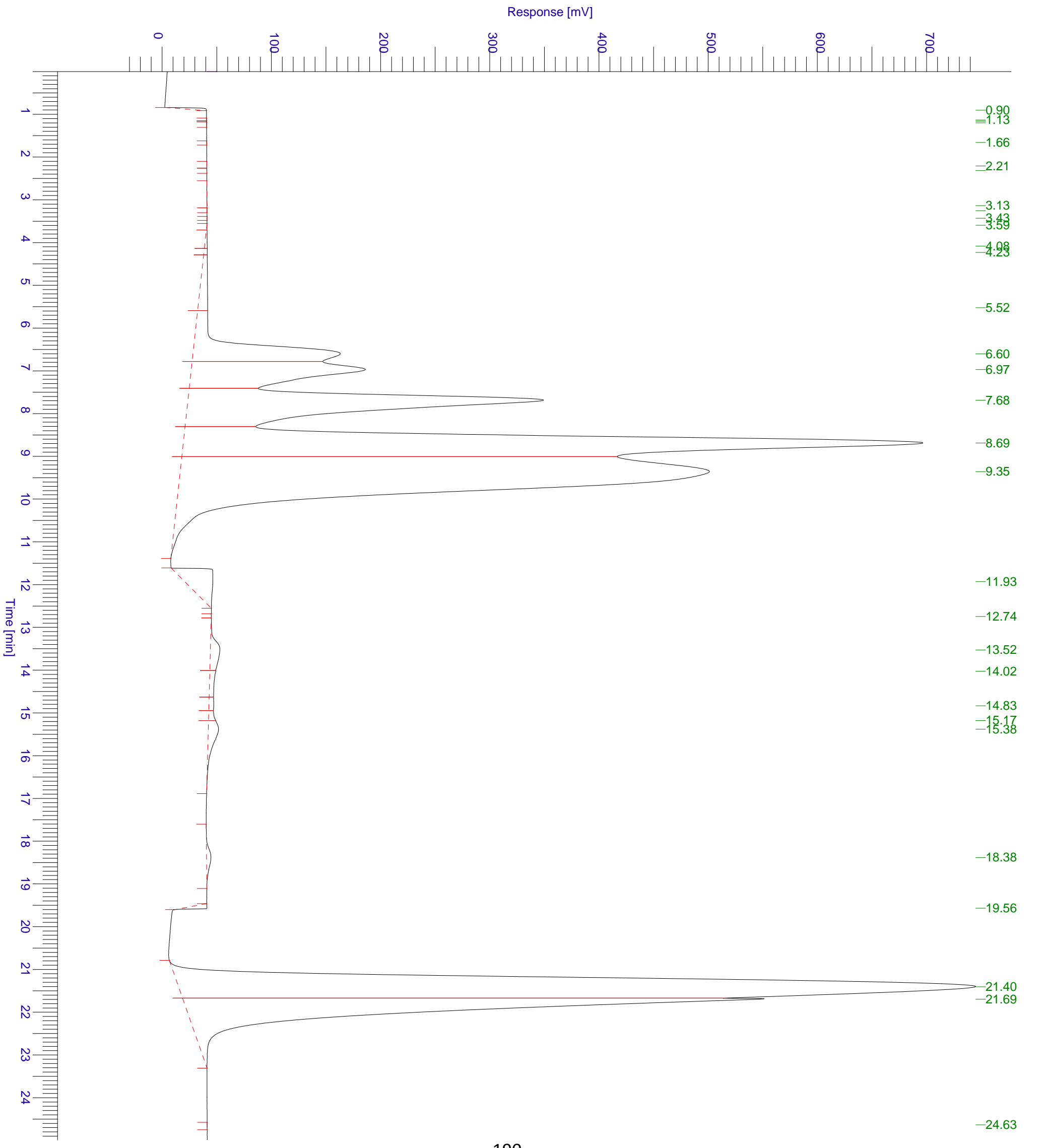




\begin{tabular}{llll}
\hline Software Version & $:$ 6.3.2.0646 & Date & $: 6 / 21 / 20132: 17: 10$ PM \\
Operator & $:$ rLuong & Sample Name & $:$ S71 \\
Sample Number & $:$ O78 & Study & $:$ Ethanol \\
AutoSampler & $:$ SER200 & Rack/Vial & $: 1 / 78$ \\
Instrument Name & $:$ HPLC & Channel & $:$ A \\
Instrument Serial \# & $:$ None & A/D mV Range $: 1000$ \\
Delay Time & $: 0.00 \mathrm{~min}$ & End Time & $: 24.99 \mathrm{~min}$ \\
Sampling Rate & $: 2.5000 \mathrm{pts} / \mathrm{s}$ & & \\
Sample Volume & $: 1.000000 \mathrm{ul}$ & & \\
Sample Amount & $: 1.0000$ & Area Reject $: 0.00000$ \\
Data Acquisition Time & $: 6 / 12 / 201311: 04: 49 \mathrm{PM}$ & Dilution Factor $: 1.00$
\end{tabular}

Raw Data File : C:IHPLC DatalrobinlEthanol_June 10-2013IEthanol078.raw

Result File : c:Ihplc datalrobinlethanol_june 10-2013lethanol078.rst [Editing in Progress]

Inst Method : C:IHPLC DatalrobinlMethod Robin 87H from C:IHPLC DatalrobinlEthanol_June 10-2013।Ethanol078.raw

Proc Method : C:IHPLC DatalrobinlMethod Robin 87H from c:Ihplc datalrobinlethanol_june 10-2013lethanol078.rst [Editing in Progress] Calib Method : C:IHPLC DatalrobinlMethod Robin 87H from c:Ihplc datalrobinlethanol_june 10-2013lethanol078.rst [Editing in Progress] Report Format File: C:IHPLC DatalrobinlMethod Robin 87H.rpt

Sequence File : C:IHPLC DatalrobinIValera Sequence 87H-Etanol_June 10_2013.seq

\begin{tabular}{|c|c|c|c|c|c|c|c|}
\hline $\begin{array}{l}\text { Peak } \\
\quad \#\end{array}$ & $\begin{array}{l}\text { Time } \\
\text { [min] }\end{array}$ & $\begin{array}{l}\text { Area } \\
{[\mu \mathrm{V} \cdot \mathrm{s}]}\end{array}$ & $\begin{array}{l}\text { Height } \\
{[\mu \mathrm{V}]}\end{array}$ & $\begin{array}{c}\text { Area } \\
{[\%]}\end{array}$ & $\begin{array}{c}\text { Norm. Area } \\
{[\%]}\end{array}$ & $B L$ & $\begin{array}{c}\text { Area/Height } \\
\text { [s] }\end{array}$ \\
\hline- & 0.001 & 0.00 & 0.00 & 0.00 & 0.00 & & \\
\hline 1 & 0.038 & 959.20 & 277.17 & 0.00 & 0.00 & BV & 3.4607 \\
\hline 2 & 0.953 & 235494.00 & 8337.34 & 0.26 & 0.26 & VB & 28.2457 \\
\hline 3 & 3.485 & 38014.60 & 1649.01 & 0.04 & 0.04 & BB & 23.0530 \\
\hline 4 & 4.083 & 443.04 & 115.48 & 0.00 & 0.00 & BV & 3.8365 \\
\hline 5 & 4.155 & 439.16 & 182.14 & 0.00 & 0.00 & VB & 2.4110 \\
\hline 6 & 4.244 & 528.05 & 130.15 & 0.00 & 0.00 & BV & 4.0573 \\
\hline 7 & 4.309 & 284.75 & 187.55 & 0.00 & 0.00 & VB & 1.5182 \\
\hline 8 & 4.393 & 430.92 & 123.86 & 0.00 & 0.00 & BV & 3.4791 \\
\hline 9 & 4.460 & 432.28 & 220.77 & 0.00 & 0.00 & VB & 1.9580 \\
\hline 10 & 4.549 & 496.43 & 133.99 & 0.00 & 0.00 & BV & 3.7049 \\
\hline 11 & 4.619 & 471.97 & 241.75 & 0.00 & 0.00 & VB & 1.9523 \\
\hline 12 & 4.709 & 618.78 & 157.25 & 0.00 & 0.00 & BV & 3.9351 \\
\hline 13 & 4.784 & 584.42 & 276.85 & 0.00 & 0.00 & VB & 2.1109 \\
\hline 14 & 4.871 & 509.59 & 124.55 & 0.00 & 0.00 & BV & 4.0916 \\
\hline 15 & 4.953 & 591.61 & 250.10 & 0.00 & 0.00 & VB & 2.3655 \\
\hline 16 & 5.048 & 342.38 & 99.00 & 0.00 & 0.00 & BV & 3.4584 \\
\hline 17 & 5.130 & 515.02 & 190.89 & 0.00 & 0.00 & VB & 2.6980 \\
\hline 18 & 5.498 & 3801.60 & 265.55 & 0.00 & 0.00 & BV & 14.3157 \\
\hline 19 & 5.568 & 980.22 & 321.10 & 0.00 & 0.00 & VV & 3.0526 \\
\hline 20 & 5.673 & 443.46 & 118.91 & 0.00 & 0.00 & VV & 3.7293 \\
\hline 21 & 5.753 & 764.33 & 218.96 & 0.00 & 0.00 & VB & 3.4906 \\
\hline 22 & 5.842 & 143.06 & 60.15 & 0.00 & 0.00 & BV & 2.3782 \\
\hline 23 & 5.948 & 529.74 & 163.83 & 0.00 & 0.00 & VB & 3.2335 \\
\hline 24 & 6.052 & 125.60 & 58.44 & 0.00 & 0.00 & $\mathrm{BB}$ & 2.1490 \\
\hline 25 & 6.598 & 2637682.68 & 123433.63 & 2.96 & 2.96 & BV & 21.3692 \\
\hline 26 & 6.970 & 3686392.74 & 142342.70 & 4.13 & 4.13 & VV & 25.8980 \\
\hline 27 & 7.684 & 7786765.29 & 300833.37 & 8.73 & 8.73 & VV & 25.8840 \\
\hline 28 & 8.689 & 17745862.99 & 700544.48 & 19.89 & 19.89 & VV & 25.3315 \\
\hline 29 & 9.358 & 25240054.90 & 510220.58 & 28.29 & 28.29 & VE & 49.4689 \\
\hline 30 & 11.300 & 15135.20 & 168.31 & 0.02 & 0.02 & EB & 89.9260 \\
\hline 31 & 11.393 & 120.20 & 123.47 & 0.00 & 0.00 & $\mathrm{BB}$ & 0.9735 \\
\hline 32 & 11.456 & 505.62 & 245.33 & 0.00 & 0.00 & BV & 2.0610 \\
\hline 33 & 11.530 & 1534.15 & 514.85 & 0.00 & 0.00 & VV & 2.9798 \\
\hline 34 & 11.584 & 1636.71 & 593.91 & 0.00 & 0.00 & VV & 2.7558 \\
\hline 35 & 11.614 & 1216.80 & 694.73 & 0.00 & 0.00 & VV & 1.7515 \\
\hline 36 & 11.664 & 2853.13 & 612.50 & 0.00 & 0.00 & VB & 4.6582 \\
\hline 37 & 11.799 & 255.48 & 129.53 & 0.00 & 0.00 & BV & 1.9723 \\
\hline 38 & 11.847 & 819.32 & 221.92 & 0.00 & 0.00 & VB & 3.6919 \\
\hline 39 & 11.968 & 1302.24 & 436.64 & 0.00 & 0.00 & BV & 2.9824 \\
\hline 40 & 12.025 & 1412.54 & 533.96 & 0.00 & 0.00 & VV & 2.6454 \\
\hline 41 & 12.051 & 409.42 & 389.76 & 0.00 & 0.00 & VB & 1.0504 \\
\hline 42 & 12.127 & 1239.01 & 463.23 & 0.00 & 0.00 & BV & 2.6747 \\
\hline 43 & 12.186 & 2339.79 & 605.44 & 0.00 & 0.00 & VV & 3.8646 \\
\hline 44 & 12.267 & 1825.66 & 499.91 & 0.00 & 0.00 & VV & 3.6520 \\
\hline 45 & 12.310 & 853.96 & 564.87 & 0.00 & 0.00 & VV & 1915118 \\
\hline
\end{tabular}


6/21/2013 2:17:10 PM Result: c:Ihplc datalrobinlethanol june 10-2013lethanol078.rst

\begin{tabular}{|c|c|c|c|c|c|c|c|}
\hline $\begin{array}{c}\text { Peak } \\
\#\end{array}$ & $\begin{array}{l}\text { Time } \\
\text { [min] }\end{array}$ & $\begin{array}{l}\text { Area } \\
{[\mu \mathrm{V} \cdot \mathrm{s}]}\end{array}$ & $\begin{array}{c}\text { Height } \\
{[\mu \mathrm{V}]}\end{array}$ & $\begin{array}{c}\text { Area } \\
{[\%]}\end{array}$ & $\begin{array}{c}\text { Norm. Area } \\
{[\%]}\end{array}$ & $B L$ & $\begin{array}{c}\text { Area/Height } \\
\text { [s] }\end{array}$ \\
\hline 46 & 12.336 & 722.78 & 525.02 & 0.00 & 0.00 & VV & 1.3767 \\
\hline 47 & 12.362 & 547.19 & 467.05 & 0.00 & 0.00 & VB & 1.1716 \\
\hline 48 & 12.448 & 2196.12 & 511.49 & 0.00 & 0.00 & BV & 4.2936 \\
\hline 49 & 12.503 & 1959.48 & 634.42 & 0.00 & 0.00 & VB & 3.0886 \\
\hline 50 & 12.620 & 9327.05 & 2086.56 & 0.01 & 0.01 & BV & 4.4701 \\
\hline 51 & 12.669 & 12688.08 & 3100.20 & 0.01 & 0.01 & VV & 4.0927 \\
\hline 52 & 12.750 & 15461.37 & 4668.81 & 0.02 & 0.02 & VV & 3.3116 \\
\hline 53 & 12.788 & 14903.60 & 5248.65 & 0.02 & 0.02 & VV & 2.8395 \\
\hline 54 & 12.843 & 24219.99 & 6125.44 & 0.03 & 0.03 & VV & 3.9540 \\
\hline 55 & 12.947 & 53367.86 & 7937.51 & 0.06 & 0.06 & VV & 6.7235 \\
\hline 56 & 13.020 & 17774.72 & 9377.60 & 0.02 & 0.02 & VV & 1.8954 \\
\hline 57 & 13.048 & 21250.75 & 9991.96 & 0.02 & 0.02 & VV & 2.1268 \\
\hline 58 & 13.116 & 38647.81 & 11282.45 & 0.04 & 0.04 & VV & 3.4255 \\
\hline 59 & 13.171 & 36195.55 & 12364.47 & 0.04 & 0.04 & VV & 2.9274 \\
\hline 60 & 13.195 & 21603.14 & 12882.64 & 0.02 & 0.02 & VV & 1.6769 \\
\hline 61 & 13.468 & 275907.99 & 20573.66 & 0.31 & 0.31 & VV & 13.4107 \\
\hline 62 & 13.488 & 37407.27 & 20996.76 & 0.04 & 0.04 & VV & 1.7816 \\
\hline 63 & 13.547 & 63388.63 & 22346.93 & 0.07 & 0.07 & VB & 2.8366 \\
\hline 64 & 16.076 & 252983.80 & 16477.62 & 0.28 & 0.28 & BB & 15.3532 \\
\hline 65 & 18.376 & 327955.20 & 10071.01 & 0.37 & 0.37 & $\mathrm{BE}$ & 32.5643 \\
\hline 66 & 19.134 & 1139.60 & 203.37 & 0.00 & 0.00 & EB & 5.6035 \\
\hline 67 & 20.154 & 605.60 & 79.11 & 0.00 & 0.00 & BB & 7.6549 \\
\hline 68 & 20.378 & 525.20 & 66.76 & 0.00 & 0.00 & BB & 7.8674 \\
\hline 69 & 20.616 & 1236.00 & 168.45 & 0.00 & 0.00 & $\mathrm{BB}$ & 7.3376 \\
\hline 70 & 21.400 & 30560026.80 & 765124.23 & 34.25 & 34.25 & MM & 39.9413 \\
\hline \multirow[t]{2}{*}{71} & 24.788 & 5365.40 & 252.22 & 0.01 & 0.01 & BB & 21.2730 \\
\hline & & 89215569.00 & $2.74 \mathrm{e}+06$ & 100.00 & 100.00 & & \\
\hline
\end{tabular}

Warning -- Signal level out-of-range in peak

Missing Component Report

Component Expected Retention (Calibration File)

standards

0.001 
Time of Injection: 6/12/2013 11:04:49 PM

Scale Factor: $1.0 \quad$ Plot Offset: $-38.92 \mathrm{mV}$

Low Point : -38.92 mV

High Point : $778.43 \mathrm{mV}$

Plot Scale: $817.4 \mathrm{mV}$

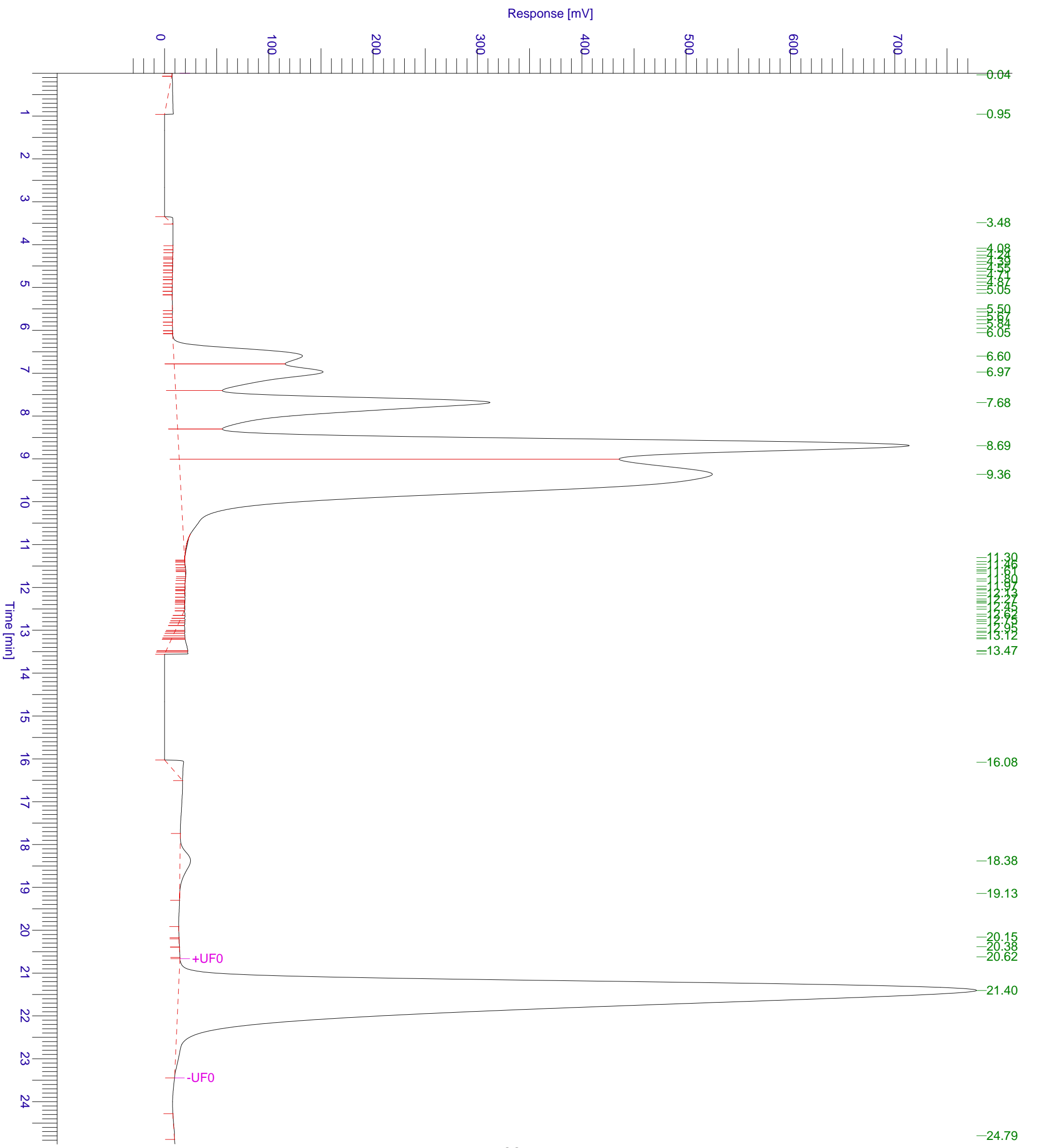




$\begin{array}{llll}\text { Software Version } & : 6.3 .2 .0646 & \text { Date } & : 6 / 21 / 20132: 18: 06 \text { PM } \\ \text { Operator } & : \text { rLuong } & \text { Sample Name } & : \text { S72 } \\ \text { Sample Number } & : \text { O79 } & \text { Study } & : \text { Ethanol } \\ \text { AutoSampler } & : \text { SER200 } & \text { Rack/Vial } & : 1 / 79 \\ \text { Instrument Name } & : \text { HPLC } & \text { Channel } & : \text { A } \\ \text { Instrument Serial \# } & : \text { None } & \text { A/D mV Range }: 1000 \\ \text { Delay Time } & : 0.00 \mathrm{~min} & \text { End Time } & : 24.99 \mathrm{~min} \\ \text { Sampling Rate } & : 2.5000 \mathrm{pts} / \mathrm{s} & & \\ \text { Sample Volume } & : 1.000000 \mathrm{ul} & & \\ \text { Sample Amount } & : 1.0000 & \text { Area Reject } & : 0.000000 \\ \text { Data Acquisition Time } & : 6 / 12 / 2013 \text { 11:31:09 PM } & \text { Dilution Factor }: 1.00\end{array}$

Raw Data File : C:IHPLC DatalrobinlEthanol_June 10-2013IEthanol079.raw

Result File : c:Ihplc datalrobinlethanol_june 10-2013lethanol079.rst [Editing in Progress]

Inst Method : C:IHPLC DatalrobinlMethod Robin 87H from C:IHPLC DatalrobinlEthanol June 10-2013।Ethanol079.raw

Proc Method : C:IHPLC DatalrobinlMethod Robin 87H from c:Ihplc datalrobinlethanol_june 10-2013lethanol079.rst [Editing in Progress] Calib Method : C:IHPLC DatalrobinlMethod Robin 87H from c:Ihplc datalrobinlethanol june 10-2013lethanol079.rst [Editing in Progress]

Report Format File: C:IHPLC DatalrobinlMethod Robin 87H.rpt

Sequence File : C:IHPLC DatalrobinIValera Sequence 87H-Etanol_June 10_2013.seq

\begin{tabular}{|c|c|c|c|c|c|c|c|}
\hline $\begin{array}{l}\text { Peak } \\
\quad \#\end{array}$ & $\begin{array}{l}\text { Time } \\
\text { [min] }\end{array}$ & $\begin{array}{l}\text { Area } \\
{[\mu \mathrm{V} \cdot \mathrm{s}]}\end{array}$ & $\begin{array}{l}\text { Height } \\
{[\mu \mathrm{V}]}\end{array}$ & $\begin{array}{c}\text { Area } \\
{[\%]}\end{array}$ & $\begin{array}{c}\text { Norm. Area } \\
{[\%]}\end{array}$ & $\mathrm{BL}$ & $\begin{array}{c}\text { Area/Height } \\
\text { [s] }\end{array}$ \\
\hline- & 0.001 & 0.00 & 0.00 & 0.00 & 0.00 & & \\
\hline 1 & 2.143 & 27121.60 & 1610.93 & 0.03 & 0.03 & BB & 16.8359 \\
\hline 2 & 2.655 & 974.20 & 84.05 & 0.00 & 0.00 & $\mathrm{BB}$ & 11.5911 \\
\hline 3 & 2.955 & 870.97 & 183.42 & 0.00 & 0.00 & BV & 4.7484 \\
\hline 4 & 3.242 & 8502.74 & 496.40 & 0.01 & 0.01 & VV & 17.1287 \\
\hline 5 & 3.558 & 9398.49 & 677.85 & 0.01 & 0.01 & VV & 13.8652 \\
\hline 6 & 3.789 & 9170.13 & 938.69 & 0.01 & 0.01 & VV & 9.7691 \\
\hline 7 & 3.959 & 13701.49 & 1216.20 & 0.02 & 0.02 & VV & 11.2658 \\
\hline 8 & 4.259 & 40975.77 & 1398.93 & 0.05 & 0.05 & VV & 29.2908 \\
\hline 9 & 5.966 & 247054.79 & 4442.06 & 0.30 & 0.30 & VV & 55.6172 \\
\hline 10 & 6.600 & 2594203.64 & 117681.22 & 3.15 & 3.15 & VV & 22.0443 \\
\hline 11 & 6.968 & 3546651.11 & 135885.84 & 4.31 & 4.31 & VV & 26.1002 \\
\hline 12 & 7.684 & 7207074.37 & 282296.01 & 8.75 & 8.75 & VV & 25.5302 \\
\hline 13 & 8.688 & 16946086.21 & 677999.26 & 20.58 & 20.58 & VV & 24.9943 \\
\hline 14 & 9.355 & 24583375.40 & 501571.23 & 29.86 & 29.86 & VV & 49.0127 \\
\hline 15 & 11.600 & 88125.43 & 6728.75 & 0.11 & 0.11 & VB & 13.0968 \\
\hline 16 & 14.237 & 108731.60 & 7276.72 & 0.13 & 0.13 & BB & 14.9424 \\
\hline 17 & 15.409 & 531828.20 & 16899.18 & 0.65 & 0.65 & BB & 31.4707 \\
\hline 18 & 16.227 & 83.00 & 39.68 & 0.00 & 0.00 & $\mathrm{BB}$ & 2.0918 \\
\hline 19 & 16.510 & 595.60 & 113.10 & 0.00 & 0.00 & $\mathrm{BB}$ & 5.2660 \\
\hline 20 & 16.850 & 2420.26 & 180.88 & 0.00 & 0.00 & BV & 13.3806 \\
\hline 21 & 16.959 & 999.74 & 138.70 & 0.00 & 0.00 & VB & 7.2080 \\
\hline 22 & 17.251 & 188.40 & 32.06 & 0.00 & 0.00 & BB & 5.8770 \\
\hline 23 & 17.479 & 280.40 & 72.14 & 0.00 & 0.00 & $\mathrm{BB}$ & 3.8871 \\
\hline 24 & 17.901 & 35050.57 & 2304.94 & 0.04 & 0.04 & BV & 15.2067 \\
\hline 25 & 18.273 & 173780.03 & 4380.68 & 0.21 & 0.21 & VB & 39.6696 \\
\hline 26 & 20.342 & 4417.22 & 382.89 & 0.01 & 0.01 & BV & 11.5365 \\
\hline 27 & 20.518 & 3478.58 & 309.45 & 0.00 & 0.00 & VB & 11.2410 \\
\hline 28 & 21.400 & 26137263.62 & 693400.25 & 31.75 & 31.75 & MM & 37.6943 \\
\hline \multirow[t]{2}{*}{29} & 24.478 & 764.40 & 92.09 & 0.00 & 0.00 & BB & 8.3006 \\
\hline & & 323167.96 & $2.46 \mathrm{e}+06$ & 100.00 & 100.00 & & \\
\hline
\end{tabular}

Warning -- Signal level out-of-range in peak

Missing Component Report

Component Expected Retention (Calibration File)

standards

0.001 
Time of Injection: 6/12/2013 11:31:09 PM

Scale Factor: $1.0 \quad$ Plot Offset: $-34.69 \mathrm{mV}$

Low Point : -34.69 mV

High Point : $693.82 \mathrm{mV}$

Plot Scale: $728.5 \mathrm{mV}$

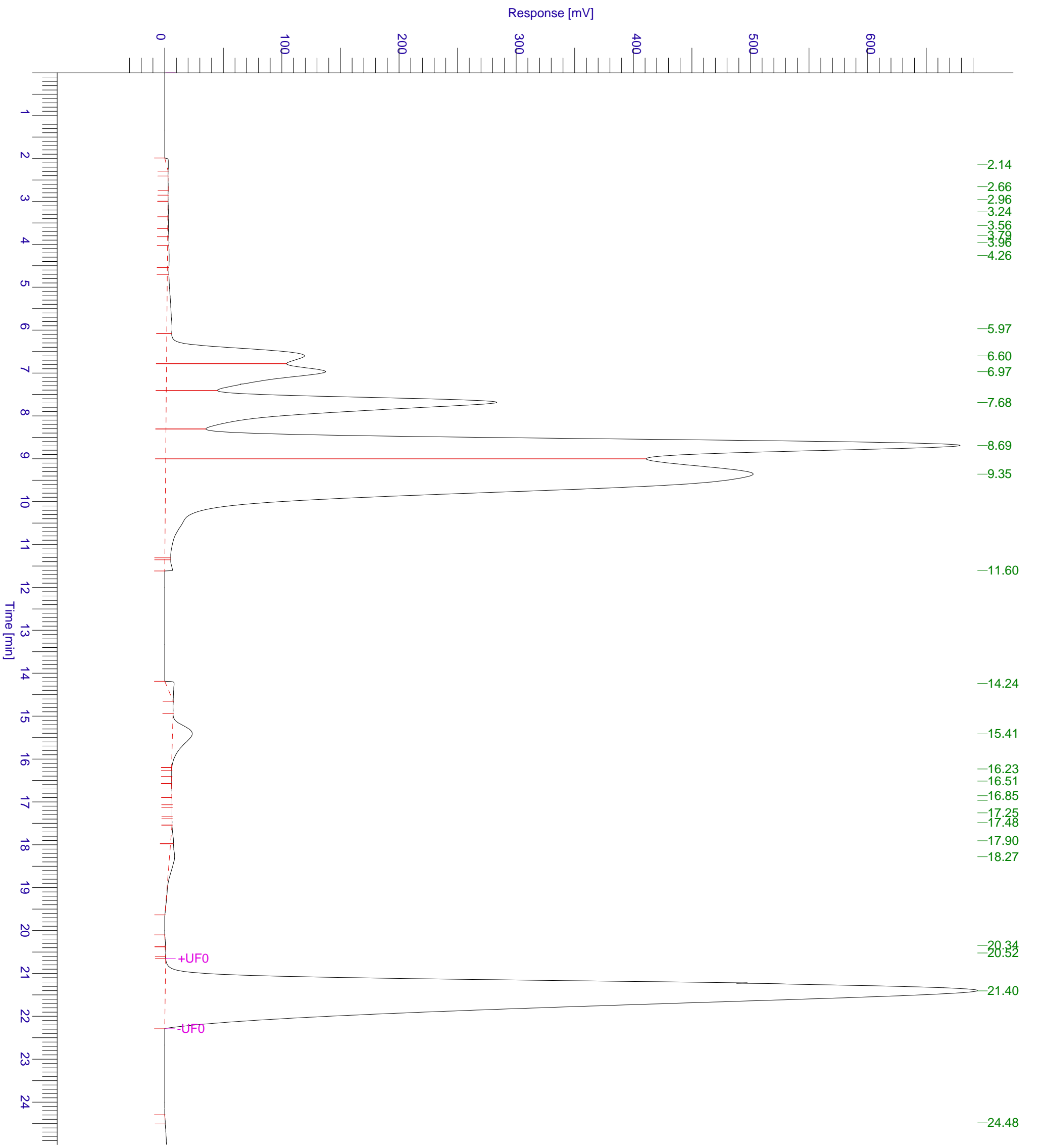




$\begin{array}{llll}\text { Software Version } & : 6.3 .2 .0646 & \text { Date } & : 6 / 21 / 20132: 19: 06 \text { PM } \\ \text { Operator } & : \text { rLuong } & \text { Sample Name } & : \text { S73 } \\ \text { Sample Number } & : \text { 080 } & \text { Study } & : \text { Ethanol } \\ \text { AutoSampler } & : \text { SER200 } & \text { Rack/Vial } & : 1 / 80 \\ \text { Instrument Name } & : \text { HPLC } & \text { Channel } & : \text { A } \\ \text { Instrument Serial \# } & : \text { None } & \text { A/D mV Range }: 1000 \\ \text { Delay Time } & : 0.00 \mathrm{~min} & \text { End Time } & : 24.99 \mathrm{~min} \\ \text { Sampling Rate } & : 2.5000 \mathrm{pts} / \mathrm{s} & & \\ \text { Sample Volume } & : 1.000000 \mathrm{ul} & & \\ \text { Sample Amount } & : 1.0000 & \text { Area Reject } & : 0.00000 \\ \text { Data Acquisition Time } & : 6 / 12 / 201311: 57: 30 \mathrm{PM} & \text { Dilution Factor }: 1.00\end{array}$

Raw Data File : C:IHPLC DatalrobinlEthanol_June 10-2013lEthanol080.raw

Result File : c:Ihplc datalrobinlethanol june 10-2013lethanol080.rst [Editing in Progress]

Inst Method : C:IHPLC DatalrobinlMethod Robin 87H from C:IHPLC DatalrobinlEthanol June 10-2013।Ethanol080.raw

Proc Method : C:IHPLC DatalrobinlMethod Robin 87H from c:Ihplc datalrobinlethanol_june 10-2013lethanol080.rst [Editing in Progress] Calib Method : C:IHPLC DatalrobinlMethod Robin 87H from c:Ihplc datalrobinlethanol june 10-2013lethanol080.rst [Editing in Progress]

Report Format File: C:IHPLC DatalrobinlMethod Robin 87H.rpt

Sequence File : C:IHPLC DatalrobinIValera Sequence 87H-Etanol_June 10_2013.seq

\begin{tabular}{|c|c|c|c|c|c|c|c|}
\hline $\begin{array}{c}\text { Peak } \\
\#\end{array}$ & $\begin{array}{l}\text { Time } \\
\text { [min] }\end{array}$ & $\begin{array}{l}\text { Area } \\
{[\mu \mathrm{V} \cdot \mathrm{s}]}\end{array}$ & $\begin{array}{l}\text { Height } \\
{[\mu \mathrm{V}]}\end{array}$ & $\begin{array}{c}\text { Area } \\
{[\%]}\end{array}$ & $\begin{array}{c}\text { Norm. Area } \\
{[\%]}\end{array}$ & $B L$ & $\begin{array}{c}\text { Area/Height } \\
\text { [s] }\end{array}$ \\
\hline - & 0.001 & 0.00 & 0.00 & 0.00 & 0.00 & & \\
\hline 1 & 1.881 & 13874.62 & 664.53 & 0.01 & 0.01 & BV & 20.8789 \\
\hline 2 & 1.991 & 3980.94 & 755.52 & 0.00 & 0.00 & VV & 5.2691 \\
\hline 3 & 2.044 & 3784.49 & 822.78 & 0.00 & 0.00 & VV & 4.5997 \\
\hline 4 & 2.172 & 4632.35 & 1131.44 & 0.00 & 0.00 & VB & 4.0942 \\
\hline 5 & 5.123 & 489078.54 & 5319.01 & 0.51 & 0.51 & BV & 91.9492 \\
\hline 6 & 5.727 & 208011.86 & 6491.43 & 0.22 & 0.22 & VB & 32.0441 \\
\hline 7 & 6.581 & 1877260.56 & 92774.00 & 1.96 & 1.96 & BV & 20.2348 \\
\hline 8 & 6.969 & 1913632.28 & 89618.86 & 2.00 & 2.00 & VV & 21.3530 \\
\hline 9 & 7.686 & 3829078.68 & 189585.60 & 4.00 & 4.00 & VV & 20.1971 \\
\hline 10 & 8.087 & 747036.36 & 64239.71 & 0.78 & 0.78 & VV & 11.6289 \\
\hline 11 & 8.704 & 18125589.57 & 709916.56 & 18.93 & 18.93 & VV & 25.5320 \\
\hline 12 & 9.466 & 33901589.67 & 658895.06 & 35.40 & 35.40 & VE & 51.4522 \\
\hline 13 & 11.792 & 991716.40 & 11513.95 & 1.04 & 1.04 & EV & 86.1317 \\
\hline 14 & 12.889 & 244855.91 & 8638.68 & 0.26 & 0.26 & VV & 28.3441 \\
\hline 15 & 13.473 & 477054.17 & 11440.94 & 0.50 & 0.50 & VB & 41.6971 \\
\hline 16 & 14.856 & 3554.52 & 608.01 & 0.00 & 0.00 & BV & 5.8461 \\
\hline 17 & 15.423 & 450994.68 & 7088.81 & 0.47 & 0.47 & VV & 63.6207 \\
\hline 18 & 16.753 & 427119.12 & 14302.58 & 0.45 & 0.45 & VB & 29.8631 \\
\hline 19 & 18.963 & 479125.00 & 16036.14 & 0.50 & 0.50 & BB & 29.8778 \\
\hline 20 & 21.413 & 31493054.91 & 794695.31 & 32.89 & 32.89 & MM & 39.6291 \\
\hline 21 & 23.548 & 33787.19 & 1569.29 & 0.04 & 0.04 & BV & 21.5302 \\
\hline 22 & 23.858 & 44407.61 & 1576.39 & 0.05 & 0.05 & VB & 28.1705 \\
\hline & & 95763219.43 & $2.69 e+06$ & 100.00 & 100.00 & & \\
\hline
\end{tabular}

Warning -- Signal level out-of-range in peak

Missing Component Report

Component Expected Retention (Calibration File)

standards

0.001 
Time of Injection: 6/12/2013 11:57:30 PM

Low Point : $-40.46 \mathrm{mV}$
Plot Scale: $849.6 \mathrm{mV}$

High Point : $809.16 \mathrm{mV}$

Response [mV]

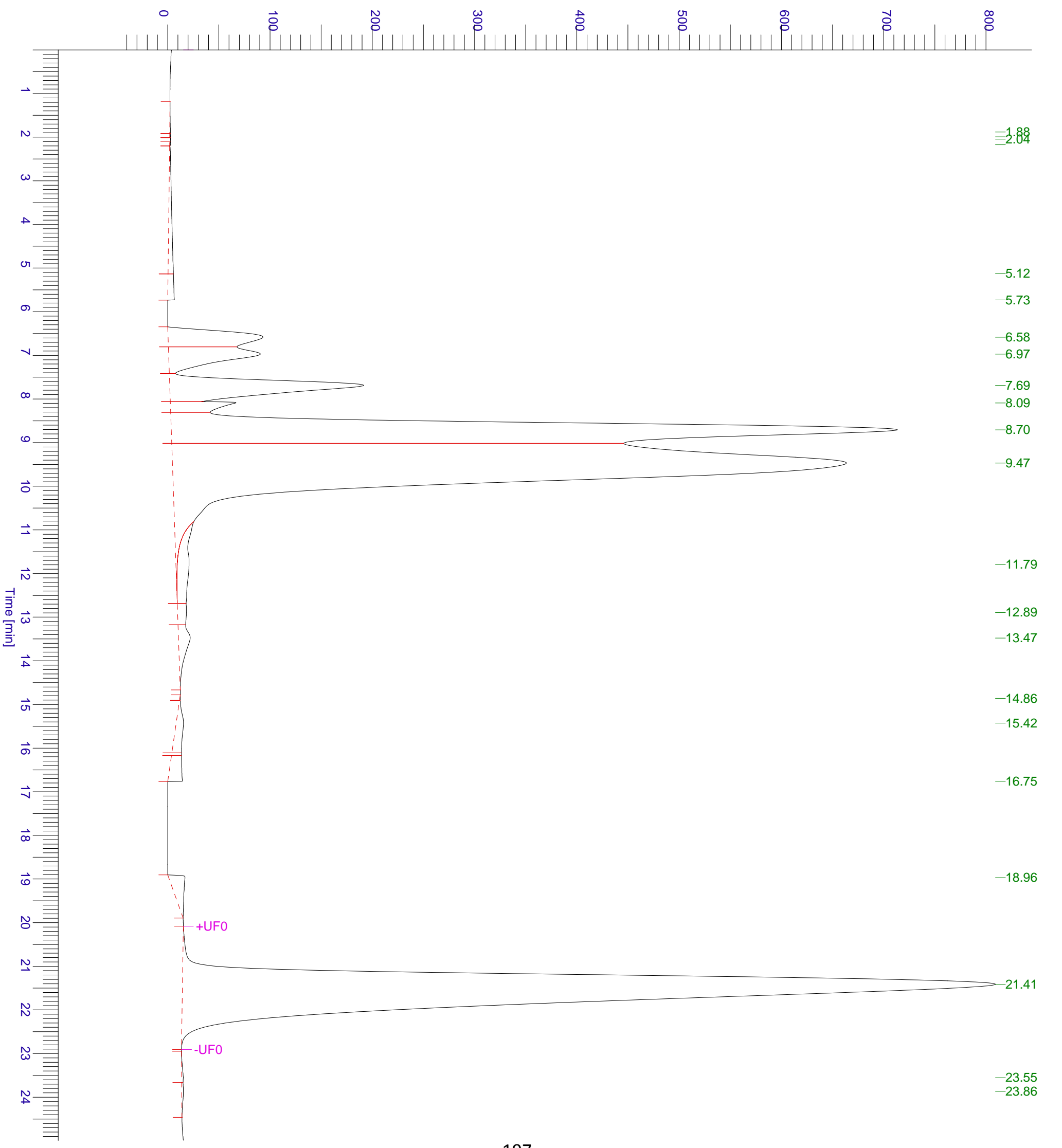




$\begin{array}{llll}\text { Software Version } & : 6.3 .2 .0646 & \text { Date } & : 6 / 21 / 20132: 20: 09 \text { PM } \\ \text { Operator } & : \text { rLuong } & \text { Sample Name } & : \text { S74 } \\ \text { Sample Number } & : \text { 081 } & \text { Study } & : \text { Ethanol } \\ \text { AutoSampler } & : \text { SER200 } & \text { Rack/Vial } & : 1 / 81 \\ \text { Instrument Name } & : \text { HPLC } & \text { Channel } & : \text { A } \\ \text { Instrument Serial \# } & : \text { None } & \text { A/D mV Range }: 1000 \\ \text { Delay Time } & : 0.00 \mathrm{~min} & \text { End Time } & : 24.99 \mathrm{~min} \\ \text { Sampling Rate } & : 2.5000 \mathrm{pts} / \mathrm{s} & & \\ \text { Sample Volume } & : 1.000000 \mathrm{ul} & & \\ \text { Sample Amount } & : 1.0000 & \text { Area Reject } & : 0.00000 \\ \text { Data Acquisition Time } & : 6 / 13 / 2013 \text { 12:23:49 AM } & \text { Dilution Factor }: 1.00\end{array}$

Raw Data File : C:IHPLC DatalrobinlEthanol_June 10-2013lEthanol081.raw

Result File : c:Ihplc datalrobinlethanol june 10-2013lethanol081.rst [Editing in Progress]

Inst Method : C:IHPLC DatalrobinlMethod Robin 87H from C:IHPLC DatalrobinlEthanol June 10-2013/Ethanol081.raw

Proc Method : C:IHPLC DatalrobinlMethod Robin 87H from c:Ihplc datalrobinlethanol june 10-2013lethanol081.rst [Editing in Progress] Calib Method : C:IHPLC DatalrobinlMethod Robin 87H from c:Ihplc datalrobinlethanol june 10-2013lethanol081.rst [Editing in Progress]

Report Format File: C:IHPLC DatalrobinlMethod Robin 87H.rpt

Sequence File : C:IHPLC DatalrobinIValera Sequence 87H-Etanol_June 10_2013.seq

\begin{tabular}{|c|c|c|c|c|c|c|c|}
\hline $\begin{array}{c}\text { Peak } \\
\#\end{array}$ & $\begin{array}{l}\text { Time } \\
\text { [min] }\end{array}$ & $\begin{array}{l}\text { Area } \\
{[\mu \mathrm{V} \cdot \mathrm{s}]}\end{array}$ & $\begin{array}{l}\text { Height } \\
{[\mu \mathrm{V}]}\end{array}$ & $\begin{array}{c}\text { Area } \\
{[\%]}\end{array}$ & $\begin{array}{c}\text { Norm. Area } \\
{[\%]}\end{array}$ & $\mathrm{BL}$ & $\begin{array}{c}\text { Area/Height } \\
\text { [s] }\end{array}$ \\
\hline- & 0.001 & 0.00 & 0.00 & 0.00 & 0.00 & & \\
\hline 1 & 1.789 & 107649.01 & 11554.55 & 0.11 & 0.11 & BV & 9.3166 \\
\hline 2 & 1.901 & 192068.08 & 11435.28 & 0.20 & 0.20 & VV & 16.7961 \\
\hline 3 & 3.879 & 868829.85 & 6880.47 & 0.91 & 0.91 & VV & 126.2748 \\
\hline 4 & 4.168 & 300968.32 & 6147.83 & 0.32 & 0.32 & VB & 48.9552 \\
\hline 5 & 5.713 & 1074.80 & 236.04 & 0.00 & 0.00 & $\mathrm{BB}$ & 4.5535 \\
\hline 6 & 6.587 & 2786875.17 & 125418.83 & 2.93 & 2.93 & $\mathrm{BV}$ & 22.2205 \\
\hline 7 & 6.969 & 3234714.60 & 126113.75 & 3.40 & 3.40 & VV & 25.6492 \\
\hline 8 & 7.687 & 6053751.31 & 233852.64 & 6.37 & 6.37 & VV & 25.8870 \\
\hline 9 & 8.698 & 17975934.72 & 711028.25 & 18.91 & 18.91 & VV & 25.2816 \\
\hline 10 & 9.473 & 31760119.79 & 593459.86 & 33.42 & 33.42 & VB & 53.5169 \\
\hline 11 & 14.027 & 86429.00 & 7077.49 & 0.09 & 0.09 & BB & 12.2118 \\
\hline 12 & 15.419 & 180107.40 & 5458.19 & 0.19 & 0.19 & $\mathrm{BB}$ & 32.9977 \\
\hline 13 & 16.830 & 378.40 & 75.29 & 0.00 & 0.00 & BB & 5.0256 \\
\hline 14 & 17.061 & 428.20 & 74.44 & 0.00 & 0.00 & $\mathrm{BB}$ & 5.7525 \\
\hline 15 & 18.401 & 154765.00 & 4301.92 & 0.16 & 0.16 & $\mathrm{BB}$ & 35.9758 \\
\hline 16 & 19.913 & 342.40 & 64.50 & 0.00 & 0.00 & $\mathrm{BB}$ & 5.3085 \\
\hline \multirow[t]{2}{*}{17} & 21.400 & 31339491.77 & 796411.21 & 32.97 & 32.97 & MM & 39.3509 \\
\hline & & 95043927.82 & $2.64 \mathrm{e}+06$ & 100.00 & 100.00 & & \\
\hline
\end{tabular}

Warning -- Signal level out-of-range in peak

Missing Component Report

Component Expected Retention (Calibration File)

standards

0.001 
Time of Injection: 6/13/2013 12:23:49 AM

Low Point : $-40.06 \mathrm{mV}$

High Point : $801.23 \mathrm{mV}$

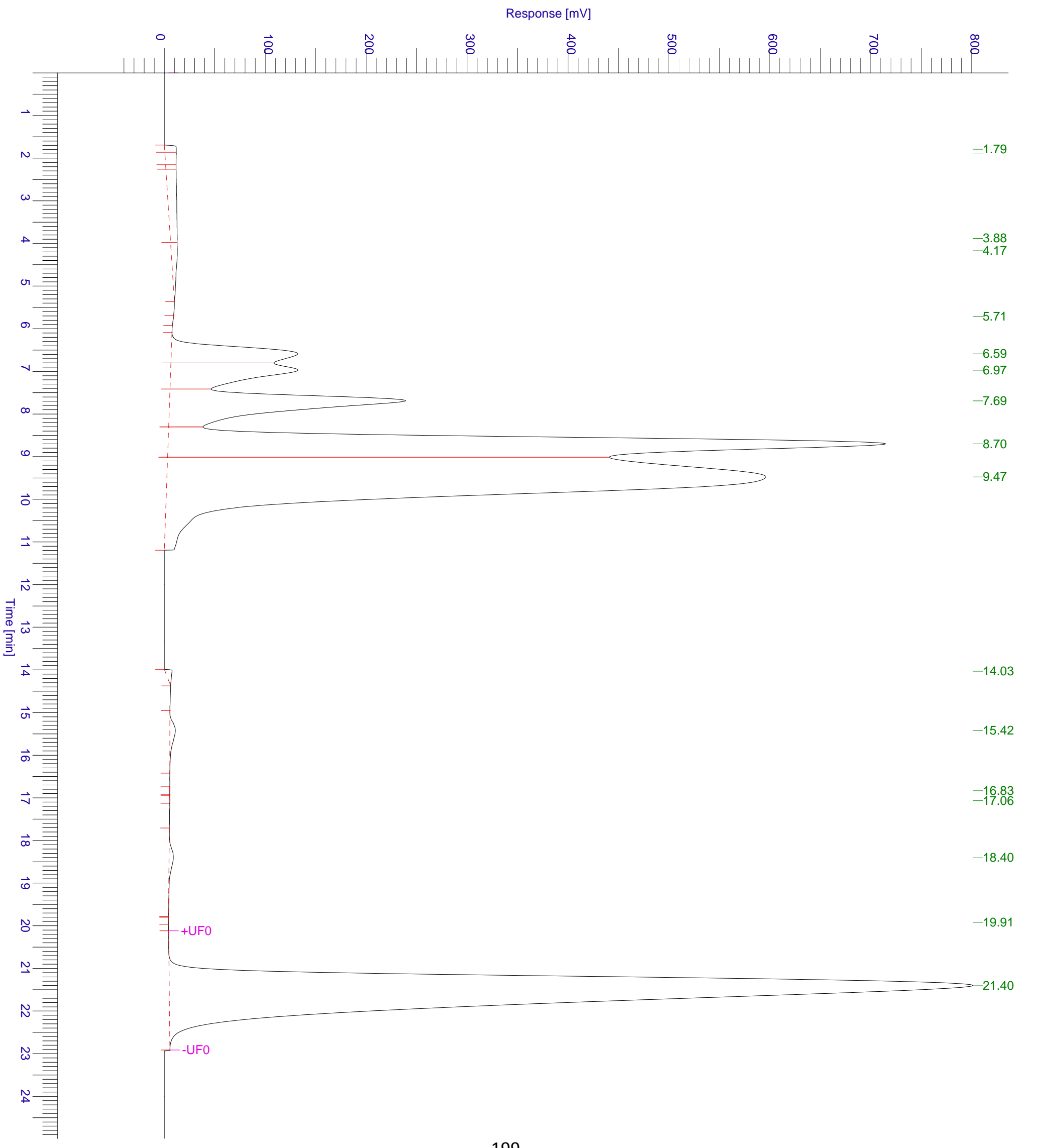




$\begin{array}{llll}\text { Software Version } & : 6.3 .2 .0646 & \text { Date } & : 6 / 21 / 20132: 21: 14 \text { PM } \\ \text { Operator } & : \text { rLuong } & \text { Sample Name } & : \text { S75 } \\ \text { Sample Number } & : \text { 082 } & \text { Study } & : \text { Ethanol } \\ \text { AutoSampler } & : \text { SER200 } & \text { Rack/Vial } & : 1 / 82 \\ \text { Instrument Name } & : \text { HPLC } & \text { Channel } & : \text { A } \\ \text { Instrument Serial \# } & : \text { None } & \text { A/D mV Range }: 1000 \\ \text { Delay Time } & : 0.00 \mathrm{~min} & \text { End Time } & : 24.99 \mathrm{~min} \\ \text { Sampling Rate } & : 2.5000 \mathrm{pts} / \mathrm{s} & & \\ \text { Sample Volume } & : 1.000000 \mathrm{ul} & & \\ \text { Sample Amount } & : 1.0000 & \text { Area Reject } & : 0.000000 \\ \text { Data Acquisition Time } & : 6 / 13 / 201312: 50: 09 \text { AM } & \text { Dilution Factor }: 1.00\end{array}$

Raw Data File : C:IHPLC DatalrobinlEthanol_June 10-2013IEthanol082.raw

Result File : c:Ihplc datalrobinlethanol_june 10-2013lethanol082.rst [Editing in Progress]

Inst Method : C:IHPLC DatalrobinlMethod Robin 87H from C:IHPLC DatalrobinlEthanol June 10-2013।Ethanol082.raw

Proc Method : C:IHPLC DatalrobinlMethod Robin 87H from c:Ihplc datalrobinlethanol_june 10-2013lethanol082.rst [Editing in Progress] Calib Method : C:IHPLC DatalrobinlMethod Robin 87H from c:Ihplc datalrobinlethanol june 10-2013lethanol082.rst [Editing in Progress]

Report Format File: C:IHPLC DatalrobinlMethod Robin 87H.rpt

Sequence File : C:IHPLC DatalrobinIValera Sequence 87H-Etanol_June 10_2013.seq

\begin{tabular}{|c|c|c|c|c|c|c|c|}
\hline $\begin{array}{c}\text { Peak } \\
\#\end{array}$ & $\begin{array}{l}\text { Time } \\
\text { [min] }\end{array}$ & $\begin{array}{l}\text { Area } \\
{[\mu \mathrm{V} \cdot \mathrm{s}]}\end{array}$ & $\begin{array}{c}\text { Height } \\
{[\mu \mathrm{V}]}\end{array}$ & $\begin{array}{c}\text { Area } \\
{[\%]}\end{array}$ & $\begin{array}{c}\text { Norm. Area } \\
{[\%]}\end{array}$ & $\mathrm{BL}$ & $\begin{array}{c}\text { Area/Height } \\
\text { [s] }\end{array}$ \\
\hline- & 0.001 & 0.00 & 0.00 & 0.00 & 0.00 & & \\
\hline 1 & 0.039 & 250.40 & 125.11 & 0.00 & 0.00 & BB & 2.0015 \\
\hline 2 & 0.181 & 1418.94 & 310.91 & 0.00 & 0.00 & $\mathrm{BV}$ & 4.5638 \\
\hline 3 & 0.372 & 3818.06 & 635.19 & 0.00 & 0.00 & VB & 6.0109 \\
\hline 4 & 0.444 & 1757.53 & 680.31 & 0.00 & 0.00 & BV & 2.5834 \\
\hline 5 & 0.538 & 4267.29 & 588.33 & 0.00 & 0.00 & VV & 7.2533 \\
\hline 6 & 1.033 & 22682.64 & 703.74 & 0.02 & 0.02 & VV & 32.2314 \\
\hline 7 & 1.316 & 997.74 & 115.48 & 0.00 & 0.00 & VB & 8.6400 \\
\hline 8 & 5.068 & 116858.22 & 3474.26 & 0.12 & 0.12 & BV & 33.6354 \\
\hline 9 & 5.588 & 130948.00 & 5273.23 & 0.14 & 0.14 & VV & 24.8326 \\
\hline 10 & 5.926 & 116977.50 & 6463.56 & 0.12 & 0.12 & VV & 18.0980 \\
\hline 11 & 6.147 & 91363.22 & 8570.20 & 0.10 & 0.10 & VB & 10.6606 \\
\hline 12 & 6.579 & 1960632.72 & 95579.19 & 2.05 & 2.05 & BV & 20.5132 \\
\hline 13 & 6.966 & 2036378.01 & 93460.10 & 2.13 & 2.13 & VV & 21.7887 \\
\hline 14 & 7.684 & 4267375.41 & 196232.48 & 4.47 & 4.47 & VV & 21.7465 \\
\hline 15 & 8.702 & 17475333.98 & 717628.93 & 18.29 & 18.29 & VV & 24.3515 \\
\hline 16 & 9.475 & 32688798.88 & 599004.65 & 34.20 & 34.20 & VE & 54.5719 \\
\hline 17 & 11.620 & 951162.40 & 10570.36 & 1.00 & 1.00 & $\mathrm{EV}$ & 89.9839 \\
\hline 18 & 13.659 & 1372101.18 & 15725.60 & 1.44 & 1.44 & VV & 87.2527 \\
\hline 19 & 15.425 & 2625097.86 & 24271.28 & 2.75 & 2.75 & VV & 108.1565 \\
\hline 20 & 17.660 & 45260.96 & 7558.00 & 0.05 & 0.05 & VB & 5.9885 \\
\hline \multirow[t]{2}{*}{21} & 21.407 & 31658412.46 & 789438.46 & 33.13 & 33.13 & MM & 40.1024 \\
\hline & & 95571893.40 & $2.58 \mathrm{e}+06$ & 00.00 & 100.00 & & \\
\hline
\end{tabular}

Warning -- Signal level out-of-range in peak

Missing Component Report

Component Expected Retention (Calibration File)

standards

0.001 
Time of Injection: 6/13/2013 12:50:09 AM

Scale Factor: $1.0 \quad$ Plot Offset: $-39.82 \mathrm{mV}$

Low Point : $-39.82 \mathrm{mV}$

High Point : $796.50 \mathrm{mV}$

Plot Scale: $836.3 \mathrm{mV}$

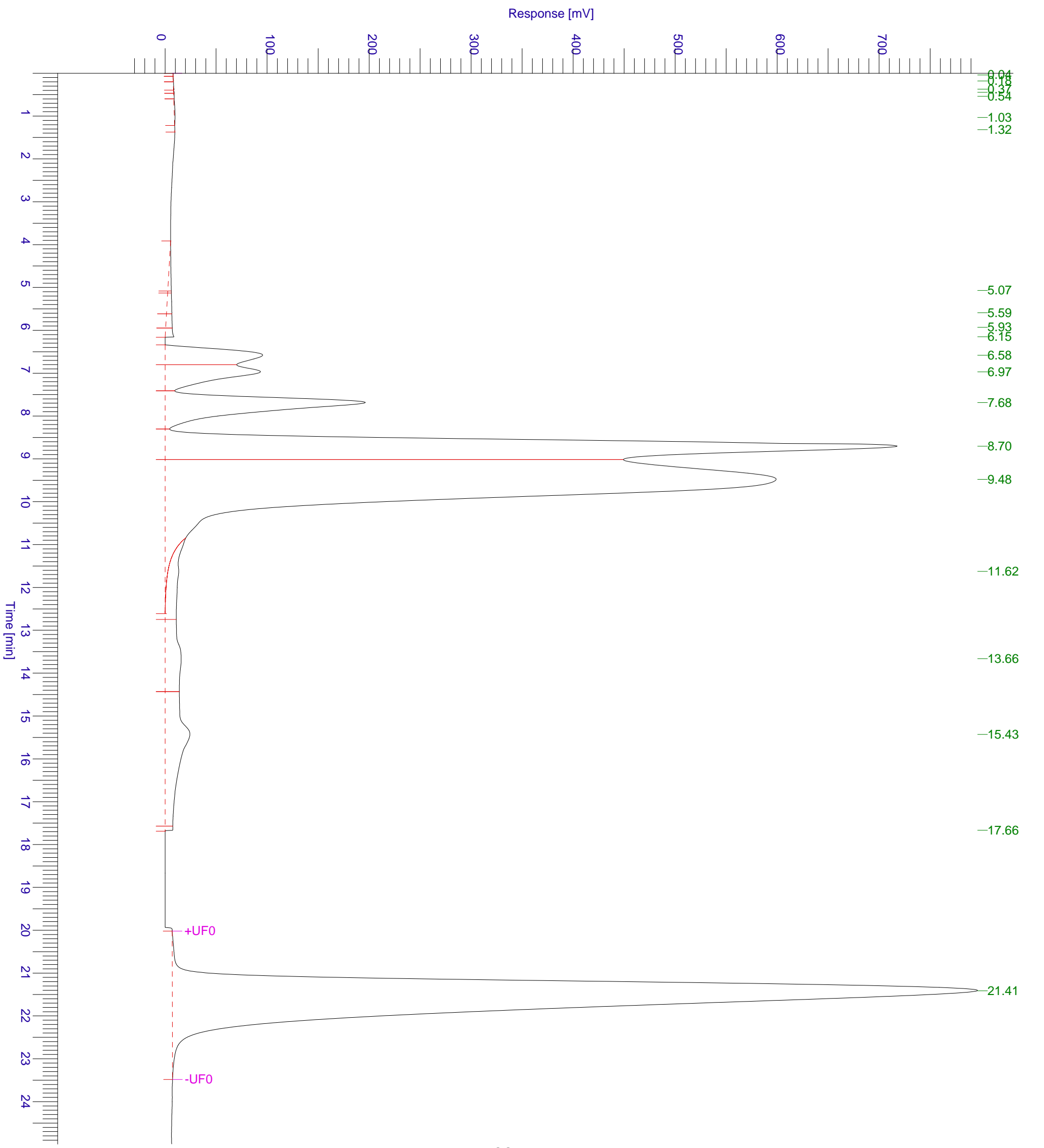




$\begin{array}{llll}\text { Software Version } & : 6.3 .2 .0646 & \text { Date } & : 6 / 21 / 20132: 22: 46 \text { PM } \\ \text { Operator } & : \text { rLuong } & \text { Sample Name } & : \text { S76 } \\ \text { Sample Number } & : \text { 083 } & \text { Study } & : \text { Ethanol } \\ \text { AutoSampler } & : \text { SER200 } & \text { Rack/Vial } & : 1 / 83 \\ \text { Instrument Name } & : \text { HPLC } & \text { Channel } & : \text { A } \\ \text { Instrument Serial \# } & : \text { None } & \text { A/D mV Range }: 1000 \\ \text { Delay Time } & : 0.00 \mathrm{~min} & \text { End Time } & : 24.99 \mathrm{~min} \\ \text { Sampling Rate } & : 2.5000 \mathrm{pts} / \mathrm{s} & & \\ \text { Sample Volume } & : 1.000000 \mathrm{ul} & & \\ \text { Sample Amount } & : 1.0000 & \text { Area Reject } & : 0.00000 \\ \text { Data Acquisition Time } & : 6 / 13 / 20131: 16: 29 \mathrm{AM} & \text { Dilution Factor }: 1.00\end{array}$

Raw Data File : C:IHPLC DatalrobinlEthanol_June 10-2013lEthanol083.raw

Result File : c:Ihplc datalrobinlethanol june 10-2013lethanol083.rst [Editing in Progress]

Inst Method : C:IHPLC DatalrobinlMethod Robin 87H from C:IHPLC DatalrobinlEthanol June 10-2013/Ethanol083.raw

Proc Method : C:IHPLC DatalrobinlMethod Robin 87H from c:Ihplc datalrobinlethanol june 10-2013lethanol083.rst [Editing in Progress] Calib Method : C:IHPLC DatalrobinlMethod Robin 87H from c:Ihplc datalrobinlethanol june 10-2013lethanol083.rst [Editing in Progress]

Report Format File: C:IHPLC DatalrobinlMethod Robin 87H.rpt

Sequence File : C:IHPLC DatalrobinIValera Sequence 87H-Etanol_June 10_2013.seq

\begin{tabular}{|c|c|c|c|c|c|c|c|}
\hline $\begin{array}{c}\text { Peak } \\
\#\end{array}$ & $\begin{array}{l}\text { Time } \\
\text { [min] }\end{array}$ & $\begin{array}{l}\text { Area } \\
{[\mu \mathrm{V} \cdot \mathrm{s}]}\end{array}$ & $\begin{array}{l}\text { Height } \\
{[\mu \mathrm{V}]}\end{array}$ & $\begin{array}{c}\text { Area } \\
{[\%]}\end{array}$ & $\begin{array}{c}\text { Norm. Area } \\
{[\%]}\end{array}$ & $B L$ & $\begin{array}{c}\text { Area/Height } \\
\text { [s] }\end{array}$ \\
\hline- & 0.001 & 0.00 & 0.00 & 0.00 & 0.00 & & \\
\hline 1 & 2.929 & 472751.04 & 6849.23 & 0.62 & 0.62 & BV & 69.0225 \\
\hline 2 & 3.074 & 59059.94 & 6140.59 & 0.08 & 0.08 & VV & 9.6180 \\
\hline 3 & 3.333 & 143983.31 & 4664.00 & 0.19 & 0.19 & VV & 30.8712 \\
\hline 4 & 3.832 & 5723.91 & 671.58 & 0.01 & 0.01 & VB & 8.5230 \\
\hline 5 & 5.798 & 12879.06 & 733.22 & 0.02 & 0.02 & BV & 17.5650 \\
\hline 6 & 6.591 & 2229977.25 & 94529.94 & 2.90 & 2.90 & VV & 23.5902 \\
\hline 7 & 6.954 & 2165560.87 & 86942.08 & 2.82 & 2.82 & VV & 24.9081 \\
\hline 8 & 7.683 & 4429474.41 & 174159.35 & 5.76 & 5.76 & VV & 25.4335 \\
\hline 9 & 8.695 & 14659402.38 & 591216.19 & 19.08 & 19.08 & VV & 24.7953 \\
\hline 10 & 9.387 & 25331466.03 & 508035.62 & 32.97 & 32.97 & VB & 49.8616 \\
\hline 11 & 14.044 & 298708.01 & 8441.92 & 0.39 & 0.39 & BV & 35.3839 \\
\hline 12 & 15.415 & 922505.14 & 20032.17 & 1.20 & 1.20 & VV & 46.0512 \\
\hline 13 & 17.327 & 84759.80 & 1552.32 & 0.11 & 0.11 & VV & 54.6021 \\
\hline 14 & 18.430 & 160170.79 & 2978.53 & 0.21 & 0.21 & VB & 53.7751 \\
\hline 15 & 19.083 & 86.20 & 44.47 & 0.00 & 0.00 & $\mathrm{BB}$ & 1.9382 \\
\hline 16 & 19.259 & 1279.00 & 70.83 & 0.00 & 0.00 & BB & 18.0572 \\
\hline 17 & 21.410 & 25798750.81 & 648138.45 & 33.58 & 33.58 & MM & 39.8044 \\
\hline \multirow[t]{2}{*}{18} & 24.556 & 61414.00 & 5310.61 & 0.08 & 0.08 & BB & 11.5644 \\
\hline & & 1.50 & $2.16 e+06$ & 100.00 & 100.00 & & \\
\hline
\end{tabular}

Missing Component Report

Component Expected Retention (Calibration File)

standards

0.001 
Time of Injection: 6/13/2013 1:16:29 AM

Start Time : $0.00 \mathrm{~min} \quad$ End Time : $24.99 \mathrm{~min}$

Low Point : $-32.56 \mathrm{mV}$

High Point : $651.32 \mathrm{mV}$

Scale Factor: $1.0 \quad$ Plot Offset: $-32.56 \mathrm{mV}$

Plot Scale: $683.9 \mathrm{mV}$

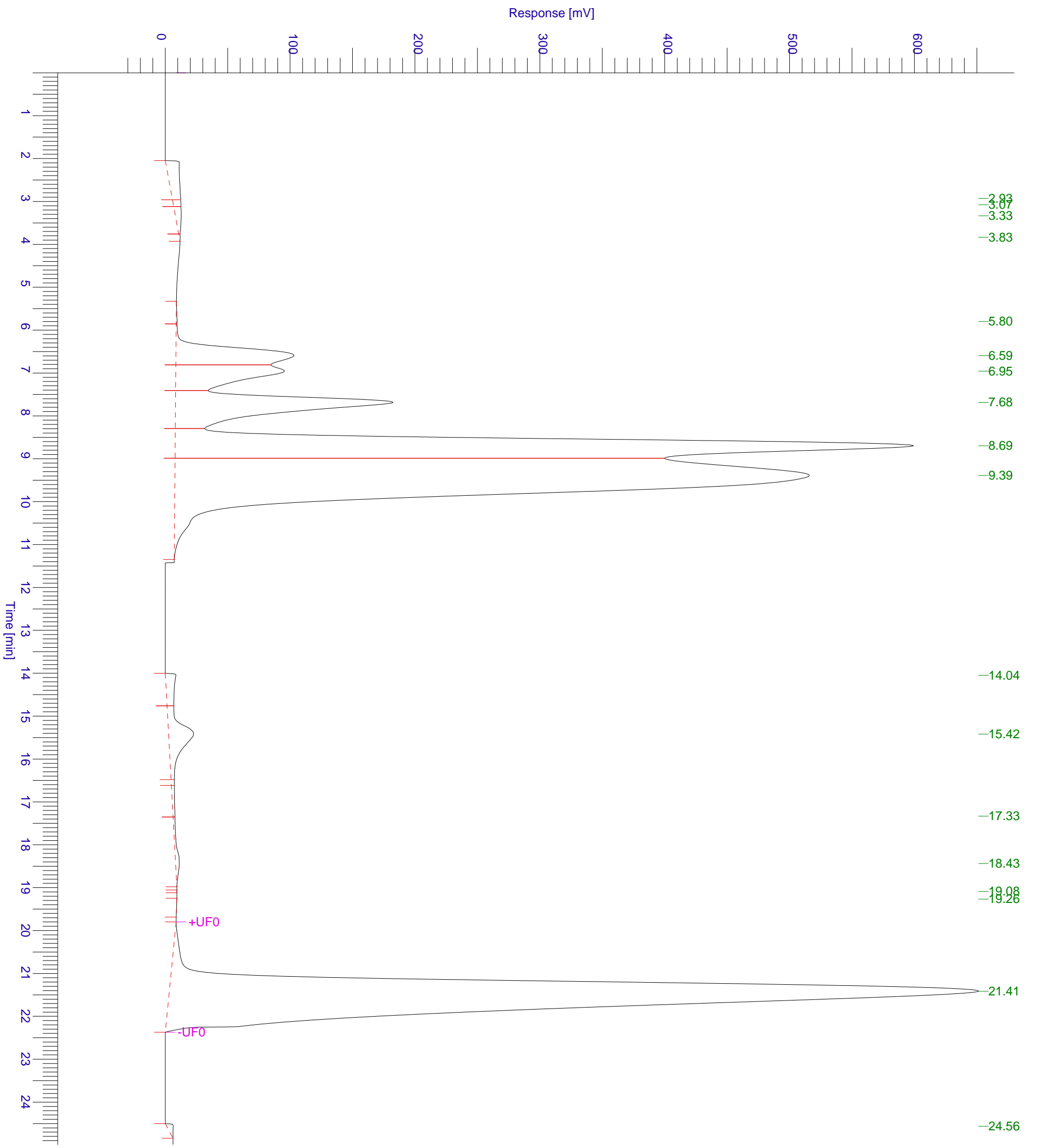




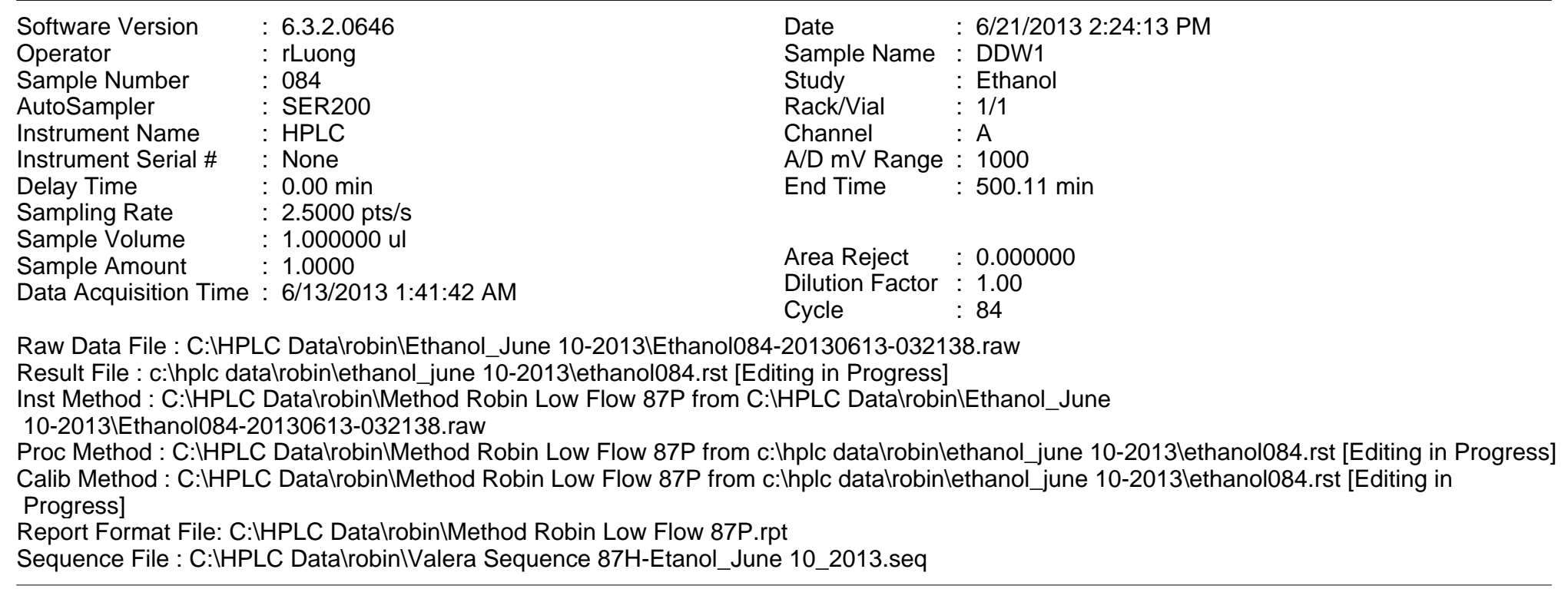

\begin{tabular}{|c|c|c|c|c|c|c|c|}
\hline $\begin{array}{c}\text { Peak } \\
\#\end{array}$ & $\begin{array}{l}\text { Time } \\
\text { [min] }\end{array}$ & $\begin{array}{c}\text { Area } \\
{[\mu \mathrm{V} \cdot \mathrm{s}]}\end{array}$ & $\begin{array}{c}\text { Height } \\
{[\mu \mathrm{V}]}\end{array}$ & $\begin{array}{c}\text { Area } \\
{[\%]}\end{array}$ & $\begin{array}{c}\text { Norm. Area } \\
{[\%]}\end{array}$ & $\mathrm{BL}$ & $\begin{array}{c}\text { Area/Height } \\
{[\mathrm{s}]}\end{array}$ \\
\hline 1 & 1.737 & 598383.67 & 3683.49 & 0.89 & 0.89 & BV & 162.4500 \\
\hline 2 & 8.371 & 2181450.33 & 9106.00 & 3.26 & 3.26 & VV & 239.5618 \\
\hline 3 & 15.067 & 1729283.60 & 9651.46 & 2.58 & 2.58 & VB & 179.1732 \\
\hline 4 & 24.560 & 513771.09 & 14507.60 & 0.77 & 0.77 & BV & 35.4139 \\
\hline 5 & 28.800 & 3450459.91 & 11283.30 & 5.15 & 5.15 & VV & 305.8024 \\
\hline 6 & 35.630 & 2752912.91 & 7701.94 & 4.11 & 4.11 & VB & 357.4310 \\
\hline 7 & 49.367 & 438852.00 & 8231.40 & 0.65 & 0.65 & BB & 53.3144 \\
\hline 8 & 54.217 & 1094832.00 & 7634.97 & 1.63 & 1.63 & $\mathrm{BB}$ & 143.3970 \\
\hline 9 & 74.853 & 1356032.80 & 11826.54 & 2.02 & 2.02 & $\mathrm{BB}$ & 114.6601 \\
\hline 10 & 91.320 & 1058878.80 & 2999.25 & 1.58 & 1.58 & $\mathrm{BB}$ & 353.0477 \\
\hline 11 & 95.891 & 1055982.10 & 8268.91 & 1.58 & 1.58 & BV & 127.7051 \\
\hline 12 & 98.805 & 3635594.99 & 14951.25 & 5.43 & 5.43 & VV & 243.1633 \\
\hline 13 & 103.593 & 4165483.30 & 24268.42 & 6.22 & 6.22 & VB & 171.6421 \\
\hline 14 & 116.769 & 2149730.00 & 13103.41 & 3.21 & 3.21 & BB & 164.0588 \\
\hline 15 & 122.533 & 982909.00 & 18212.32 & 1.47 & 1.47 & BB & 53.9695 \\
\hline 16 & 135.180 & 556382.40 & 14922.90 & 0.83 & 0.83 & $\mathrm{BB}$ & 37.2838 \\
\hline 17 & 141.493 & 118848.40 & 1233.73 & 0.18 & 0.18 & BB & 96.3323 \\
\hline 18 & 160.973 & 2562830.83 & 11232.37 & 3.82 & 3.82 & BV & 228.1647 \\
\hline 19 & 164.767 & 651247.97 & 2257.09 & 0.97 & 0.97 & VB & 288.5342 \\
\hline 20 & 178.867 & 808412.00 & 7519.79 & 1.21 & 1.21 & BB & 107.5046 \\
\hline 21 & 198.580 & 3183128.00 & 3220.05 & 4.75 & 4.75 & BB & 988.5336 \\
\hline 22 & 209.653 & 19740348.00 & 94348.00 & 29.46 & 29.46 & BB & 209.2291 \\
\hline 23 & 212.127 & 626527.20 & 4426.26 & 0.94 & 0.94 & BB & 141.5477 \\
\hline 24 & 231.793 & 308673.80 & 7966.20 & 0.46 & 0.46 & $\mathrm{BB}$ & 38.7479 \\
\hline 25 & 251.413 & 741608.40 & 5472.60 & 1.11 & 1.11 & BB & 135.5129 \\
\hline 26 & 270.567 & 900839.60 & 5101.14 & 1.34 & 1.34 & $\mathrm{BB}$ & 176.5956 \\
\hline 27 & 288.813 & 277665.60 & 7571.82 & 0.41 & 0.41 & $\mathrm{BB}$ & 36.6709 \\
\hline 28 & 304.151 & 490448.40 & 9356.95 & 0.73 & 0.73 & $\mathrm{BB}$ & 52.4154 \\
\hline 29 & 324.907 & 798483.20 & 976.32 & 1.19 & 1.19 & $\mathrm{BB}$ & 817.8504 \\
\hline 30 & 337.067 & 285767.60 & 5021.44 & 0.43 & 0.43 & $\mathrm{BB}$ & 56.9094 \\
\hline 31 & 358.820 & 1159072.00 & 798.75 & 1.73 & 1.73 & BB & 1451.1101 \\
\hline 32 & 376.387 & 1206408.40 & 737.11 & 1.80 & 1.80 & BB & 1636.6798 \\
\hline 33 & 388.560 & 127715.60 & 4637.69 & 0.19 & 0.19 & $\mathrm{BB}$ & 27.5386 \\
\hline 34 & 391.433 & 24790.40 & 489.55 & 0.04 & 0.04 & BB & 50.6390 \\
\hline 35 & 408.220 & 1152236.20 & 4082.84 & 1.72 & 1.72 & $\mathrm{BB}$ & 282.2141 \\
\hline 36 & 432.526 & 923327.00 & 4816.08 & 1.38 & 1.38 & $\mathrm{BB}$ & 191.7177 \\
\hline 37 & 444.593 & 303966.40 & 6294.50 & 0.45 & 0.45 & $\mathrm{BB}$ & 48.2908 \\
\hline 38 & 454.967 & 1293316.80 & 8435.90 & 1.93 & 1.93 & $\mathrm{BB}$ & 153.3111 \\
\hline 39 & 469.633 & 1361289.20 & 3421.97 & 2.03 & 2.03 & $\mathrm{BB}$ & 397.8082 \\
\hline \multirow[t]{2}{*}{40} & 485.973 & 237178.40 & 3977.84 & 0.35 & 0.35 & BB & 59.6250 \\
\hline & & & & & 100.00 & & \\
\hline
\end{tabular}


6/21/2013 2:24:13 PM Result: c:Ihplc datalrobinlethanol_june 10-2013lethanol084.rst

Warning -- Signal level out-of-range in peak

Missing Component Report

Component Expected Retention (Calibration File)

All components were found 
Time of Injection: 6/13/2013 1:41:42 AM

Low Point : $-4.72 \mathrm{mV}$

High Point : $94.35 \mathrm{mV}$

Plot Scale: $99.1 \mathrm{mV}$

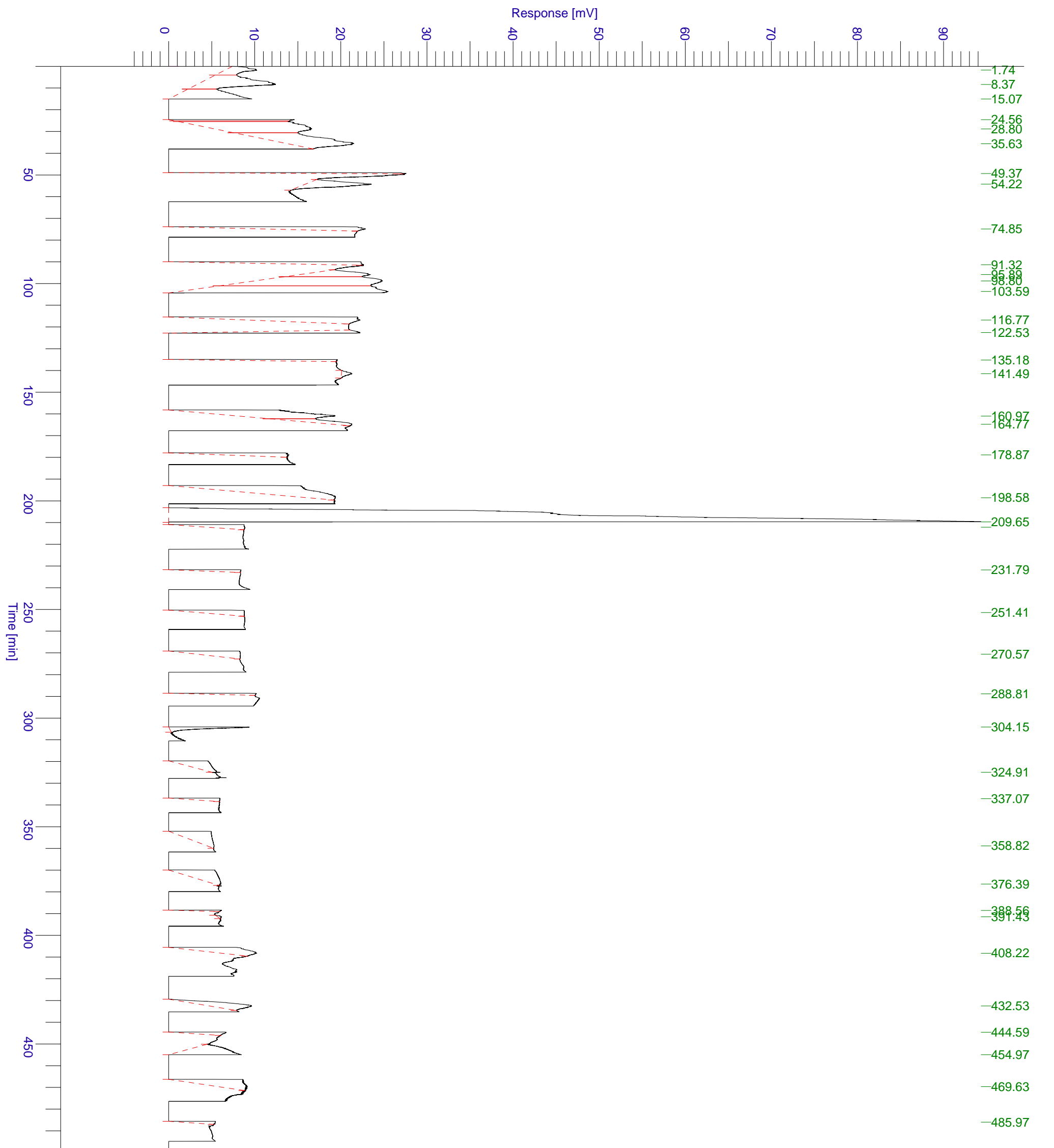




$\begin{array}{llll}\text { Software Version } & : 6.3 .2 .0646 & \text { Date } & : 6 / 21 / 201312: 56: 22 \text { PM } \\ \text { Operator } & : \text { rLuong } & \text { Sample Name } & : \text { S8 } \\ \text { Sample Number } & : \text { O15 } & \text { Study } & : \text { Ethanol } \\ \text { AutoSampler } & : \text { SER200 } & \text { Rack/Vial } & : 1 / 15 \\ \text { Instrument Name } & : \text { HPLC } & \text { Channel } & : \text { A } \\ \text { Instrument Serial \# } & : \text { None } & \text { A/D mV Range }: 1000 \\ \text { Delay Time } & : 0.00 \mathrm{~min} & \text { End Time } & : 24.99 \mathrm{~min} \\ \text { Sampling Rate } & : 2.5000 \mathrm{pts} / \mathrm{s} & & \\ \text { Sample Volume } & : 1.000000 \mathrm{ul} & & \\ \text { Sample Amount } & : 1.0000 & \text { Area Reject } & : 0.000000 \\ \text { Data Acquisition Time } & : 6 / 11 / 2013 \text { 7:27:41 PM } & \text { Dilution Factor }: 1.00\end{array}$

Raw Data File : C:IHPLC DatalrobinlEthanol_June 10-2013IEthanol015.raw

Result File : c:Ihplc datalrobinlethanol_june 10-2013lethanol015.rst [Editing in Progress]

Inst Method : C:IHPLC DatalrobinlMethod Robin 87H from C:IHPLC DatalrobinlEthanol_June 10-2013।Ethanol015.raw

Proc Method : C:IHPLC DatalrobinlMethod Robin 87H from c:Ihplc datalrobinlethanol_june 10-2013lethanol015.rst [Editing in Progress] Calib Method : C:IHPLC DatalrobinlMethod Robin 87H from c:Ihplc datalrobinlethanol_june 10-2013lethanol015.rst [Editing in Progress]

Report Format File: C:IHPLC DatalrobinlMethod Robin 87H.rpt

Sequence File : C:IHPLC DatalrobinIValera Sequence 87H-Etanol_June 10_2013.seq

\begin{tabular}{|c|c|c|c|c|c|c|c|}
\hline $\begin{array}{l}\text { Peak } \\
\quad \#\end{array}$ & $\begin{array}{l}\text { Time } \\
\text { [min] }\end{array}$ & $\begin{array}{l}\text { Area } \\
{[\mu \mathrm{V} \cdot \mathrm{s}]}\end{array}$ & $\begin{array}{c}\text { Height } \\
{[\mu \mathrm{V}]}\end{array}$ & $\begin{array}{c}\text { Area } \\
{[\%]}\end{array}$ & $\begin{array}{c}\text { Norm. Area } \\
{[\%]}\end{array}$ & $B L$ & $\begin{array}{c}\text { Area/Height } \\
{[\mathrm{s}]}\end{array}$ \\
\hline - & 0.001 & 0.00 & 0.00 & 0.00 & 0.00 & & \\
\hline 1 & 0.110 & 131.60 & 59.19 & 0.00 & 0.00 & BB & 2.2234 \\
\hline 2 & 0.263 & 120.00 & 79.21 & 0.00 & 0.00 & BB & 1.5150 \\
\hline 3 & 0.362 & 87.20 & 48.60 & $8 e-05$ & $8 e-05$ & BB & 1.7942 \\
\hline 4 & 0.474 & 69.46 & 72.15 & $6 e-05$ & $6 e-05$ & BV & 0.9626 \\
\hline 5 & 0.501 & 120.54 & 87.98 & 0.00 & 0.00 & VB & 1.3701 \\
\hline 6 & 0.735 & 342.60 & 91.07 & 0.00 & 0.00 & BB & 3.7621 \\
\hline 7 & 0.941 & 369.60 & 121.34 & 0.00 & 0.00 & BB & 3.0460 \\
\hline 8 & 1.162 & 67.00 & 65.09 & $6 e-05$ & $6 e-05$ & BB & 1.0293 \\
\hline 9 & 1.237 & 99.60 & 49.87 & $9 e-05$ & $9 e-05$ & BB & 1.9972 \\
\hline 10 & 1.393 & 580.60 & 148.88 & 0.00 & 0.00 & BB & 3.8998 \\
\hline 11 & 1.539 & 83.00 & 60.47 & $7 e-05$ & $7 e-05$ & BB & 1.3726 \\
\hline 12 & 1.649 & 220.00 & 73.63 & 0.00 & 0.00 & BB & 2.9880 \\
\hline 13 & 1.919 & 167.20 & 93.53 & 0.00 & 0.00 & BB & 1.7877 \\
\hline 14 & 2.026 & 358.80 & 86.64 & 0.00 & 0.00 & BB & 4.1412 \\
\hline 15 & 2.201 & 524.00 & 98.94 & 0.00 & 0.00 & BV & 5.2959 \\
\hline 16 & 2.342 & 352.00 & 78.27 & 0.00 & 0.00 & VB & 4.4975 \\
\hline 17 & 2.660 & 85.00 & 53.91 & $8 e-05$ & $8 e-05$ & BB & 1.5768 \\
\hline 18 & 2.987 & 264.00 & 52.88 & 0.00 & 0.00 & BB & 4.9922 \\
\hline 19 & 3.153 & 113.80 & 57.08 & 0.00 & 0.00 & BB & 1.9938 \\
\hline 20 & 3.566 & 131.20 & 57.62 & 0.00 & 0.00 & BB & 2.2771 \\
\hline 21 & 4.431 & 246.40 & 66.27 & 0.00 & 0.00 & BB & 3.7183 \\
\hline 22 & 5.582 & 61.60 & 51.31 & $5 e-05$ & $5 e-05$ & BB & 1.2006 \\
\hline 23 & 5.729 & 129.60 & 58.24 & 0.00 & 0.00 & BB & 2.2254 \\
\hline 24 & 5.882 & 272.20 & 111.92 & 0.00 & 0.00 & BB & 2.4321 \\
\hline 25 & 6.566 & 2988995.59 & 108708.12 & 2.67 & 2.67 & BV & 27.4956 \\
\hline 26 & 7.301 & 3205027.29 & 86463.83 & 2.86 & 2.86 & VV & 37.0678 \\
\hline 27 & 8.605 & 16994992.43 & 693895.75 & 15.16 & 15.16 & VV & 24.4 \\
\hline 28 & 9.267 & 55802536.10 & 985875.96 & 49.79 & 49.79 & VB & 56.6020 \\
\hline 29 & 12.771 & 280.40 & 112.76 & 0.00 & 0.00 & BB & 2.4867 \\
\hline 30 & 13.384 & 3482.00 & 173.43 & 0.00 & 0.00 & BB & 20.0775 \\
\hline 31 & 14.088 & 386.40 & 133.70 & 0.00 & 0.00 & BB & 2.8901 \\
\hline 32 & 14.386 & 613.40 & 135.80 & 0.00 & 0.00 & BB & 4.5170 \\
\hline 33 & 14.676 & 102.03 & 124.79 & $9 e-05$ & $9 e-05$ & $\mathrm{BV}$ & 0.8176 \\
\hline 34 & 14.701 & 395.97 & 126.28 & 0.00 & 0.00 & VB & 3.1357 \\
\hline 35 & 14.927 & 888.78 & 157.81 & 0.00 & 0.00 & BV & 5.6319 \\
\hline 36 & 14.961 & 310.20 & 201.43 & 0.00 & 0.00 & VV & 1.5400 \\
\hline 37 & 15.380 & 134317.90 & 4529.43 & 0.12 & 0.12 & VV & 29.6545 \\
\hline 38 & 15.860 & 6289.92 & 888.98 & 0.01 & 0.01 & VB & 7.0755 \\
\hline 39 & 16.152 & 501.48 & 210.06 & 0.00 & 0.00 & BV & 2.3874 \\
\hline 40 & 16.220 & 103.92 & 53.81 & $9 e-05$ & $9 e-05$ & VB & 1.9311 \\
\hline 41 & 16.436 & 131.80 & 82.88 & 0.00 & 0.00 & BB & 1.590 \\
\hline 42 & 16.706 & 311.60 & 126.33 & 0.00 & 0.00 & BB & 2.466 \\
\hline 43 & 16.922 & 72.40 & 58.69 & $6 e-05$ & $6 e-05$ & BB & 1.233 \\
\hline 44 & 16.984 & 344.80 & 119.99 & 0.00 & 0.00 & BB & \\
\hline 45 & 17.444 & 196.89 & 56.19 & 0.00 & 0.00 & BV & 26.5037 \\
\hline
\end{tabular}


6/21/2013 12:56:22 PM Result: c:Ihplc datalrobinlethanol_june 10-2013lethanol015.rst

\begin{tabular}{|c|c|c|c|c|c|c|c|}
\hline $\begin{array}{c}\text { Peak } \\
\text { \# }\end{array}$ & $\begin{array}{l}\text { Time } \\
\text { [min] }\end{array}$ & $\begin{array}{l}\text { Area } \\
{[\mu \mathrm{V} \cdot \mathrm{s}]}\end{array}$ & $\begin{array}{l}\text { Height } \\
{[\mu \mathrm{V}]}\end{array}$ & $\begin{array}{c}\text { Area } \\
{[\%]}\end{array}$ & $\begin{array}{c}\text { Norm. Area } \\
{[\%]}\end{array}$ & $B L$ & $\begin{array}{c}\text { Area/Height } \\
\text { [s] }\end{array}$ \\
\hline 46 & 18.314 & 102413.11 & 2532.85 & 0.09 & 0.09 & VB & 40.4340 \\
\hline 47 & 19.217 & 214.40 & 91.34 & 0.00 & 0.00 & BB & 2.3472 \\
\hline 48 & 19.465 & 132.00 & 67.68 & 0.00 & 0.00 & BB & 1.9504 \\
\hline 49 & 20.261 & 812.12 & 158.44 & 0.00 & 0.00 & BV & 5.1257 \\
\hline 50 & 20.527 & 4733.13 & 310.38 & 0.00 & 0.00 & VV & 15.2493 \\
\hline 51 & 21.518 & 32820006.20 & 819878.16 & 29.28 & 29.28 & VB & 40.0303 \\
\hline 52 & 24.169 & 174.80 & 64.44 & 0.00 & 0.00 & BB & 2.7127 \\
\hline 53 & 24.342 & 375.20 & 69.43 & 0.00 & 0.00 & BB & 5.4043 \\
\hline 54 & 24.819 & 86.80 & 35.13 & $8 e-05$ & $8 e-05$ & BB & 2.4711 \\
\hline \multirow[t]{2}{*}{55} & 24.938 & 192.20 & 82.38 & 0.00 & 0.00 & BB & 2.3331 \\
\hline & & $1.12 \mathrm{e}+0$ & $2.71 e+06$ & L00.00 & 100.00 & & \\
\hline
\end{tabular}

Warning -- Signal level out-of-range in peak

Missing Component Report

Component Expected Retention (Calibration File)

standards

0.001 
Time of Injection: 6/11/2013 7:27:41 PM

Scale Factor: $1.0 \quad$ Plot Offset: $-43.48 \mathrm{mV}$

Low Point : $-43.48 \mathrm{mV}$

Plot Scale: $1043.5 \mathrm{mV}$

High Point : $1000.00 \mathrm{mV}$

Response [mV]

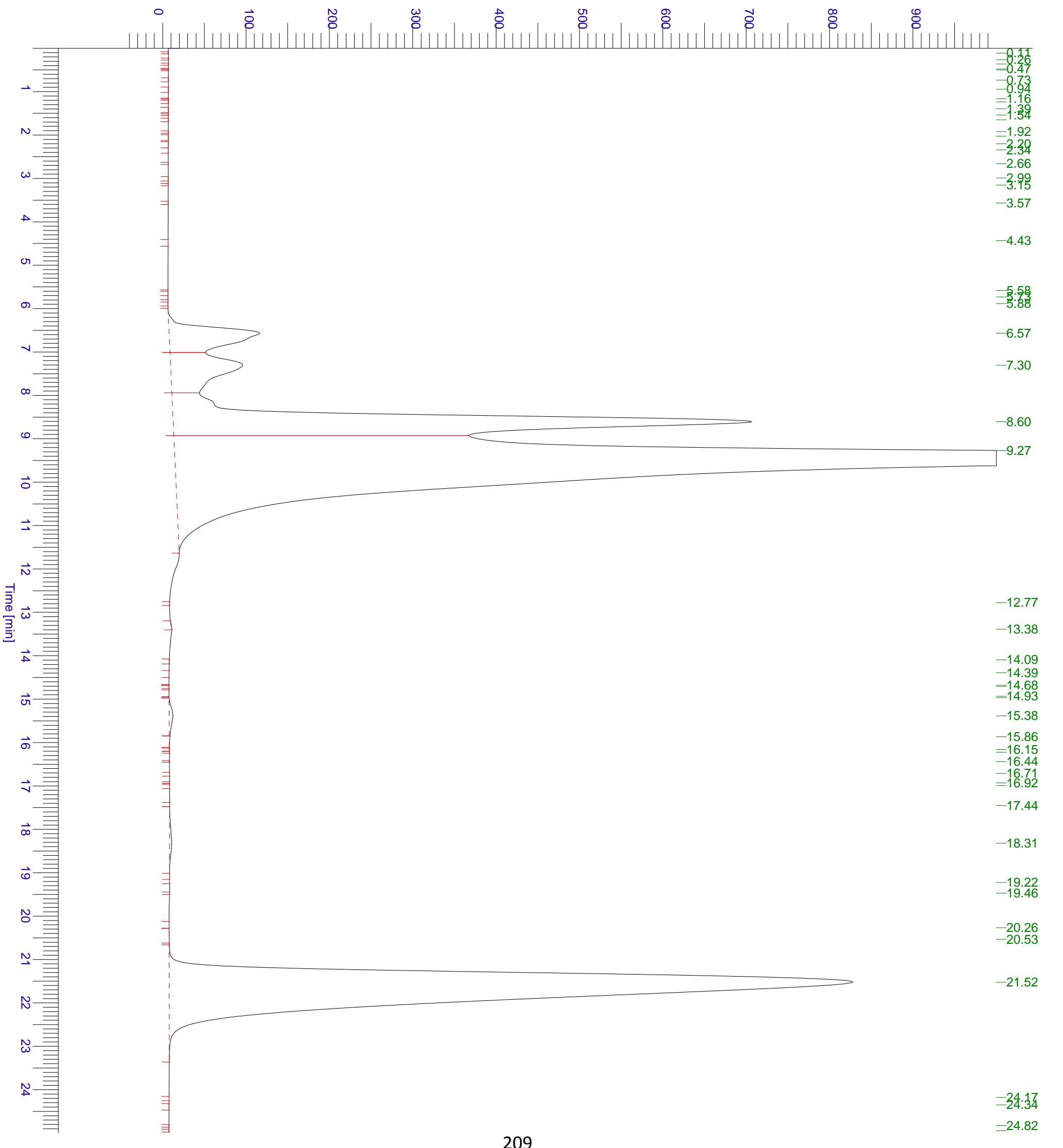




$\begin{array}{llll}\text { Software Version } & : 6.3 .2 .0646 & \text { Date } & : 6 / 21 / 201312: 57: 06 \text { PM } \\ \text { Operator } & : \text { rLuong } & \text { Sample Name } & : \text { S9 } \\ \text { Sample Number } & : \text { O16 } & \text { Study } & : \text { Ethanol } \\ \text { AutoSampler } & : \text { SER200 } & \text { Rack/Vial } & : 1 / 16 \\ \text { Instrument Name } & : \text { HPLC } & \text { Channel } & : \text { A } \\ \text { Instrument Serial \# } & : \text { None } & \text { A/D mV Range }: 1000 \\ \text { Delay Time } & : 0.00 \mathrm{~min} & \text { End Time } & : 24.99 \mathrm{~min} \\ \text { Sampling Rate } & : 2.5000 \mathrm{pts} / \mathrm{s} & & \\ \text { Sample Volume } & : 1.000000 \mathrm{ul} & & \\ \text { Sample Amount } & : 1.0000 & \text { Area Reject } & : 0.000000 \\ \text { Data Acquisition Time } & : 6 / 11 / 2013 \text { 7:53:57 PM } & \text { Dilution Factor }: 1.00\end{array}$

Raw Data File : C:IHPLC DatalrobinlEthanol_June 10-2013IEthanol016.raw

Result File : c:Ihplc datalrobinlethanol_june 10-2013lethanol016.rst [Editing in Progress]

Inst Method : C:IHPLC DatalrobinlMethod Robin 87H from C:IHPLC DatalrobinlEthanol_June 10-2013।Ethanol016.raw

Proc Method : C:IHPLC DatalrobinlMethod Robin 87H from c:Ihplc datalrobinlethanol_june 10-2013lethanol016.rst [Editing in Progress] Calib Method : C:IHPLC DatalrobinlMethod Robin 87H from c:Ihplc datalrobinlethanol_june 10-2013lethanol016.rst [Editing in Progress] Report Format File: C:IHPLC DatalrobinlMethod Robin 87H.rpt

Sequence File : C:IHPLC DatalrobinIValera Sequence 87H-Etanol_June 10_2013.seq

\begin{tabular}{|c|c|c|c|c|c|c|c|}
\hline $\begin{array}{l}\text { Peak } \\
\quad \#\end{array}$ & $\begin{array}{l}\text { Time } \\
\text { [min] }\end{array}$ & $\begin{array}{l}\text { Area } \\
{[\mu \mathrm{V} \cdot \mathrm{s}]}\end{array}$ & $\begin{array}{l}\text { Height } \\
{[\mu \mathrm{V}]}\end{array}$ & $\begin{array}{c}\text { Area } \\
{[\%]}\end{array}$ & $\begin{array}{c}\text { Norm. Area } \\
{[\%]}\end{array}$ & $B L$ & $\begin{array}{c}\text { Area/Height } \\
\text { [s] }\end{array}$ \\
\hline - & 0.001 & 0.00 & 0.00 & 0.00 & 0.00 & & \\
\hline 1 & 0.186 & 154.80 & 53.80 & 0.00 & 0.00 & BB & 2.8774 \\
\hline 2 & 0.245 & 226.40 & 89.26 & 0.00 & 0.00 & BB & 2.5366 \\
\hline 3 & 0.321 & 354.40 & 131.00 & 0.00 & 0.00 & BB & 2.7053 \\
\hline 4 & 0.517 & 146.41 & 88.26 & 0.00 & 0.00 & BV & 1.6589 \\
\hline 5 & 0.569 & 132.39 & 76.65 & 0.00 & 0.00 & VB & 1.7271 \\
\hline 6 & 0.813 & 788.40 & 175.08 & 0.00 & 0.00 & BB & 4.5032 \\
\hline 7 & 1.096 & 891.00 & 116.84 & 0.00 & 0.00 & BB & 7.6260 \\
\hline 8 & 1.256 & 124.91 & 66.03 & 0.00 & 0.00 & BV & 1.8918 \\
\hline 9 & 1.315 & 171.59 & 109.06 & 0.00 & 0.00 & VB & 1.5733 \\
\hline 10 & 1.372 & 164.20 & 81.44 & 0.00 & 0.00 & BB & 2.0161 \\
\hline 11 & 1.562 & 100.61 & 95.76 & $9 e-05$ & $9 e-05$ & BV & 1.0507 \\
\hline 12 & 1.595 & 138.99 & 96.92 & 0.00 & 0.00 & VB & 1.4340 \\
\hline 13 & 1.759 & 94.19 & 93.85 & $8 e-05$ & $8 e-05$ & BV & 1.0036 \\
\hline 14 & 1.788 & 131.41 & 88.85 & 0.00 & 0.00 & VB & 1.4790 \\
\hline 15 & 1.817 & 54.00 & 75.86 & $5 e-05$ & $5 e-05$ & BB & 0.7119 \\
\hline 16 & 1.850 & 276.00 & 128.83 & 0.00 & 0.00 & BB & 2.1424 \\
\hline 17 & 1.944 & 163.80 & 44.20 & 0.00 & 0.00 & BB & 3.7056 \\
\hline 18 & 2.207 & 139.60 & 87.59 & 0.00 & 0.00 & BB & 1.5938 \\
\hline 19 & 2.439 & 584.35 & 114.93 & 0.00 & 0.00 & $\mathrm{BV}$ & 5.0843 \\
\hline 20 & 2.528 & 127.25 & 63.85 & 0.00 & 0.00 & VB & 1.9931 \\
\hline 21 & 2.835 & 126.82 & 62.41 & 0.00 & 0.00 & BV & 2.0321 \\
\hline 22 & 2.913 & 211.93 & 75.31 & 0.00 & 0.00 & VV & 2.8140 \\
\hline 23 & 2.939 & 112.85 & 103.79 & 0.00 & 0.00 & VB & 1.0873 \\
\hline 24 & 2.965 & 107.00 & 107.56 & $1 e-04$ & $1 e-04$ & BB & 0.9948 \\
\hline 25 & 3.005 & 290.00 & 154.11 & 0.00 & 0.00 & BB & 1.8818 \\
\hline 26 & 3.169 & 210.58 & 89.03 & 0.00 & 0.00 & BV & 2.3651 \\
\hline 27 & 3.220 & 149.42 & 68.02 & 0.00 & 0.00 & VB & 2.1969 \\
\hline 28 & 3.440 & 172.80 & 92.45 & 0.00 & 0.00 & BB & 1.8691 \\
\hline 29 & 3.490 & 101.06 & 81.65 & $9 e-05$ & $9 e-05$ & BV & 1.2378 \\
\hline 30 & 3.528 & 428.34 & 103.90 & 0.00 & 0.00 & VB & 4.1224 \\
\hline 31 & 3.609 & 87.77 & 84.48 & $8 e-05$ & $8 e-05$ & BV & 1.0390 \\
\hline 32 & 3.645 & 291.78 & 142.41 & 0.00 & 0.00 & VV & 2.0489 \\
\hline 33 & 3.693 & 381.85 & 109.21 & 0.00 & 0.00 & VB & 3.4963 \\
\hline 34 & 4.181 & 542.80 & 178.71 & 0.00 & 0.00 & BB & 3.0374 \\
\hline 35 & 4.294 & 56.80 & 59.12 & $5 e-05$ & $5 e-05$ & BB & 0.9607 \\
\hline 36 & 4.332 & 203.60 & 96.91 & 0.00 & 0.00 & BB & 2.1008 \\
\hline 37 & 4.542 & 328.40 & 117.90 & 0.00 & 0.00 & BB & 2.7853 \\
\hline 38 & 4.731 & 698.00 & 90.27 & 0.00 & 0.00 & BB & 7.7325 \\
\hline 39 & 4.961 & 181.20 & 75.93 & 0.00 & 0.00 & BB & 2.3865 \\
\hline 40 & 5.018 & 728.00 & 218.82 & 0.00 & 0.00 & BB & 3.3269 \\
\hline 41 & 5.285 & 182.80 & 84.34 & 0.00 & 0.00 & BB & 2.1675 \\
\hline 42 & 5.475 & 494.40 & 109.92 & 0.00 & 0.00 & BB & 4.4977 \\
\hline 43 & 5.701 & 96.80 & 76.09 & $9 e-05$ & $9 e-05$ & BB & 1.2722 \\
\hline 44 & 5.732 & 53.20 & 55.33 & $5 e-05$ & $5 e-05$ & BB & 0.9615 \\
\hline 45 & 5.795 & 221.60 & 71.51 & 0.00 & 0.00 & BB & 23. \\
\hline
\end{tabular}




\begin{tabular}{|c|c|c|c|c|c|c|c|}
\hline $\begin{array}{c}\text { Peak } \\
\#\end{array}$ & $\begin{array}{l}\text { Time } \\
\text { [min] }\end{array}$ & $\begin{array}{l}\text { Area } \\
{[\mu \mathrm{V} \cdot \mathrm{s}]}\end{array}$ & $\begin{array}{l}\text { Height } \\
{[\mu \mathrm{V}]}\end{array}$ & $\begin{array}{c}\text { Area } \\
{[\%]}\end{array}$ & $\begin{array}{c}\text { Norm. Area } \\
{[\%]}\end{array}$ & BL & $\begin{array}{c}\text { Area/Height } \\
{[\mathrm{s}]}\end{array}$ \\
\hline 46 & 6.014 & 566.12 & 194.74 & 0.00 & 0.00 & BV & 2.9071 \\
\hline 47 & 6.570 & 3062468.13 & 108498.92 & 2.74 & 2.74 & VV & 28.2258 \\
\hline 48 & 7.302 & 3352790.61 & 89893.32 & 3.00 & 3.00 & VV & 37.2974 \\
\hline 49 & 8.614 & 17473367.32 & 693450.42 & 15.64 & 15.64 & VV & 25.1977 \\
\hline 50 & 9.280 & 55286945.02 & 997493.21 & 49.47 & 49.47 & VB & 55.4259 \\
\hline 51 & 16.666 & 15013.20 & 2253.60 & 0.01 & 0.01 & BB & 6.6619 \\
\hline 52 & 16.870 & 274.00 & 72.69 & 0.00 & 0.00 & BB & 3.7696 \\
\hline 53 & 16.942 & 682.59 & 199.08 & 0.00 & 0.00 & BV & 3.4288 \\
\hline 54 & 17.040 & 169.75 & 63.66 & 0.00 & 0.00 & VV & 2.6667 \\
\hline 55 & 17.087 & 2140.93 & 336.68 & 0.00 & 0.00 & VV & 6.3589 \\
\hline 56 & 17.234 & 634.06 & 214.93 & 0.00 & 0.00 & VV & 2.9501 \\
\hline 57 & 17.287 & 93.35 & 88.34 & $8 e-05$ & $8 e-05$ & VV & 1.0568 \\
\hline 58 & 17.353 & 686.72 & 140.71 & 0.00 & 0.00 & VB & 4.8805 \\
\hline 59 & 17.537 & 442.15 & 127.98 & 0.00 & 0.00 & BV & 3.4549 \\
\hline 60 & 17.594 & 139.05 & 94.32 & 0.00 & 0.00 & VB & 1.4744 \\
\hline 61 & 17.639 & 232.22 & 129.60 & 0.00 & 0.00 & BV & 1.7918 \\
\hline 62 & 17.681 & 608.66 & 169.84 & 0.00 & 0.00 & VV & 3.5837 \\
\hline 63 & 18.273 & 35618.89 & 1965.37 & 0.03 & 0.03 & VV & 18.1232 \\
\hline 64 & 18.307 & 36891.95 & 2020.01 & 0.03 & 0.03 & VV & 18.2633 \\
\hline 65 & 18.770 & 804.84 & 437.36 & 0.00 & 0.00 & VV & 1.8402 \\
\hline 66 & 18.791 & 1634.63 & 419.62 & 0.00 & 0.00 & VB & 3.8955 \\
\hline 67 & 18.915 & 101.80 & 71.50 & $9 e-05$ & $9 e-05$ & BV & 1.4238 \\
\hline 68 & 18.957 & 101.00 & 76.29 & $9 e-05$ & $9 e-05$ & VB & 1.3239 \\
\hline 69 & 18.999 & 181.20 & 130.43 & 0.00 & 0.00 & BV & 1.3892 \\
\hline 70 & 19.048 & 474.80 & 169.47 & 0.00 & 0.00 & VB & 2.8016 \\
\hline 71 & 19.240 & 1139.25 & 193.78 & 0.00 & 0.00 & BV & 5.8791 \\
\hline 72 & 19.303 & 382.35 & 81.39 & 0.00 & 0.00 & VB & 4.6976 \\
\hline 73 & 19.676 & 100.99 & 133.74 & $9 e-05$ & $9 e-05$ & BV & 0.7552 \\
\hline 74 & 19.702 & 1384.41 & 179.24 & 0.00 & 0.00 & VB & 7.7239 \\
\hline 75 & 20.046 & 43.80 & 46.54 & $4 e-05$ & $4 e-05$ & BB & 0.9412 \\
\hline 76 & 20.167 & 328.18 & 93.95 & 0.00 & 0.00 & BV & 3.4931 \\
\hline 77 & 20.246 & 299.04 & 101.67 & 0.00 & 0.00 & VV & 2.9412 \\
\hline 78 & 20.311 & 57.38 & 71.70 & $5 e-05$ & $5 e-05$ & VB & 0.8004 \\
\hline 79 & 20.345 & 523.04 & 131.46 & 0.00 & 0.00 & BV & 3.9786 \\
\hline 80 & 20.449 & 421.31 & 152.71 & 0.00 & 0.00 & VV & 2.7590 \\
\hline 81 & 20.571 & 321.85 & 98.87 & 0.00 & 0.00 & VB & 3.2555 \\
\hline 82 & 20.604 & 98.00 & 73.31 & $9 e-05$ & $9 e-05$ & BB & 1.3367 \\
\hline 83 & 20.781 & 1923.65 & 405.76 & 0.00 & 0.00 & BV & 4.7409 \\
\hline 84 & 21.529 & 32456699.35 & 810278.62 & 29.04 & 29.04 & VB & 40.0562 \\
\hline 85 & 23.451 & 390.00 & 141.40 & 0.00 & 0.00 & BB & 2.7582 \\
\hline 86 & 23.706 & 68.20 & 71.41 & $6 e-05$ & $6 e-05$ & BB & 0.9551 \\
\hline 87 & 23.742 & 243.20 & 98.11 & 0.00 & 0.00 & BB & 2.4787 \\
\hline 88 & 24.022 & 272.80 & 94.37 & 0.00 & 0.00 & BB & 2.8908 \\
\hline 89 & 24.168 & 373.60 & 56.62 & 0.00 & 0.00 & BB & 6.5985 \\
\hline 90 & 24.287 & 60.60 & 62.39 & $5 e-05$ & $5 e-05$ & BB & 0.9713 \\
\hline 91 & 24.327 & 156.00 & 61.68 & 0.00 & 0.00 & BB & 2.5290 \\
\hline 92 & 24.586 & 65.80 & 66.27 & $6 e-05$ & $6 e-05$ & BB & 0.9929 \\
\hline 93 & 24.619 & 107.00 & 68.05 & $1 e-04$ & $1 e-04$ & BB & 1.5723 \\
\hline 94 & 24.742 & 188.80 & 54.67 & 0.00 & 0.00 & BB & 3.4536 \\
\hline 95 & 24.889 & 69.08 & 65.11 & $6 e-05$ & $6 e-05$ & BV & 1.0609 \\
\hline \multirow[t]{2}{*}{96} & 24.924 & 174.12 & 113.84 & 0.00 & 0.00 & VB & 1.5295 \\
\hline & & 1.12 & 06 & & & & \\
\hline
\end{tabular}

Warning -- Signal level out-of-range in peak

Missing Component Report

Component Expected Retention (Calibration File)

standards

0.001 
Time of Injection: 6/11/2013 7:53:57 PM

Start Time : $0.00 \mathrm{~min} \quad$ End Time : $24.99 \mathrm{~min}$

Low Point : $-50.00 \mathrm{mV}$

Scale Factor: 1.0

Plot Offset: $-50.00 \mathrm{mV}$

Plot Scale: $1050.0 \mathrm{mV}$

High Point : $1000.00 \mathrm{mV}$

Response [mV]

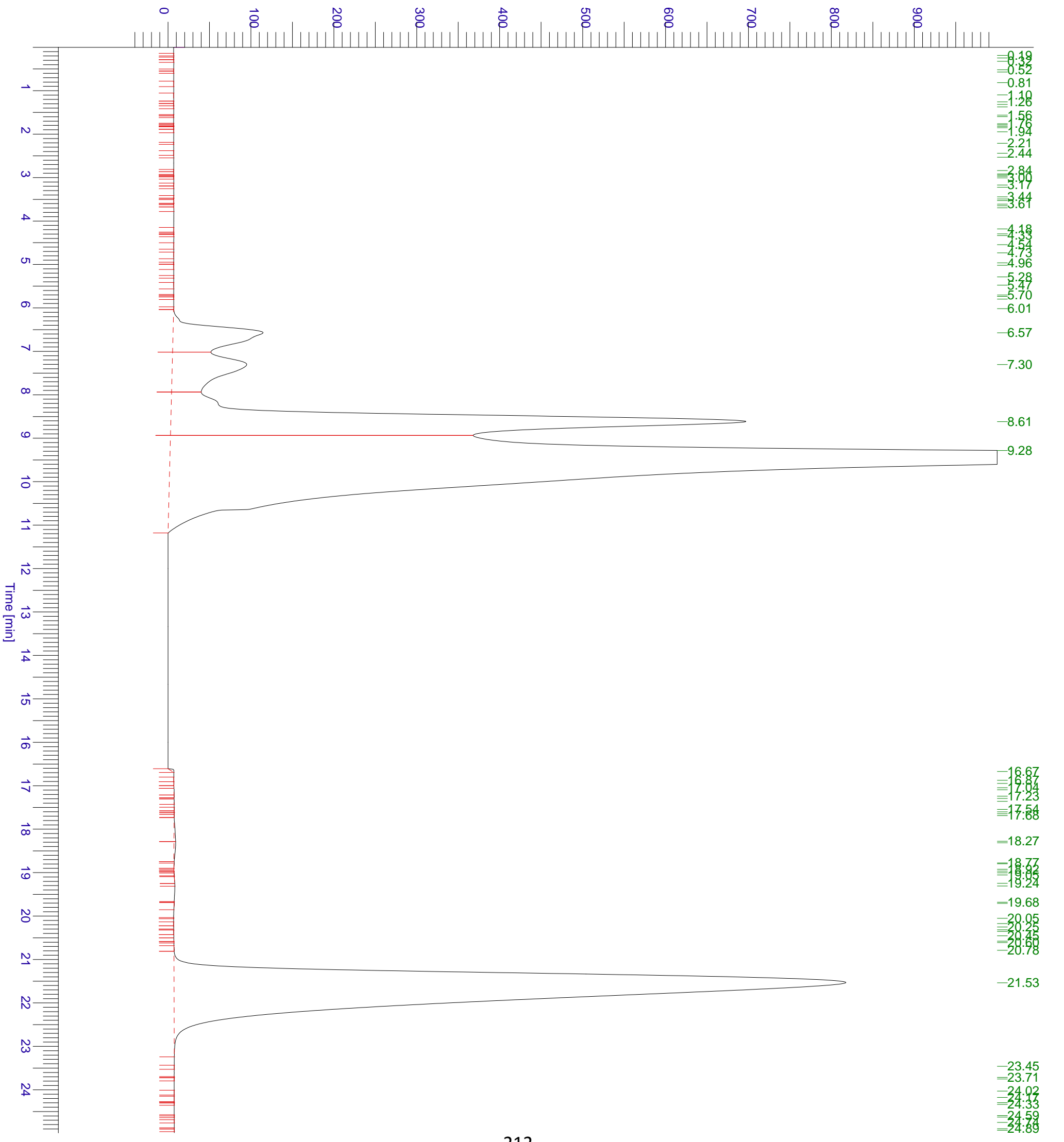




$\begin{array}{llll}\text { Software Version } & : 6.3 .2 .0646 & \text { Date } & : 6 / 21 / 201312: 41: 08 \text { PM } \\ \text { Operator } & : \text { rLuong } & \text { Sample Name } & : \text { St1 } \\ \text { Sample Number } & : 003 & \text { Study } & : \text { Ethanol } \\ \text { AutoSampler } & : \text { SER200 } & \text { Rack/Vial } & : 1 / 3 \\ \text { Instrument Name } & : \text { HPLC } & \text { Channel } & : \text { A } \\ \text { Instrument Serial \# } & : \text { None } & \text { A/D mV Range }: 1000 \\ \text { Delay Time } & : 0.00 \mathrm{~min} & \text { End Time } & : 24.99 \mathrm{~min} \\ \text { Sampling Rate } & : 2.5000 \mathrm{pts} / \mathrm{s} & & \\ \text { Sample Volume } & : 1.000000 \mathrm{ul} & & \\ \text { Sample Amount } & : 1.0000 & \text { Area Reject } & : 0.000000 \\ \text { Data Acquisition Time } & : 6 / 11 / 20132: 12: 29 \mathrm{PM} & \text { Dilution Factor }: 1.00\end{array}$

Raw Data File : C:IHPLC DatalrobinlEthanol_June 10-2013IEthanol003.raw

Result File : c:Ihplc datalrobinlethanol_june 10-2013lethanol003.rst [Editing in Progress]

Inst Method : C:IHPLC DatalrobinlMethod Robin 87H from C:IHPLC DatalrobinlEthanol_June 10-2013।Ethanol003.raw

Proc Method : C:IHPLC DatalrobinlMethod Robin 87H from c:Ihplc datalrobinlethanol_june 10-2013lethanol003.rst [Editing in Progress] Calib Method : C:IHPLC DatalrobinlMethod Robin 87H from c:Ihplc datalrobinlethanol_june 10-2013lethanol003.rst [Editing in Progress]

Report Format File: C:IHPLC DatalrobinlMethod Robin 87H.rpt

Sequence File : C:IHPLC DatalrobinIValera Sequence 87H-Etanol_June 10_2013.seq

\begin{tabular}{|c|c|c|c|c|c|c|c|}
\hline $\begin{array}{c}\text { Peak } \\
\text { \# }\end{array}$ & $\begin{array}{l}\text { Time } \\
\text { [min] }\end{array}$ & $\begin{array}{l}\text { Area } \\
{[\mu \mathrm{N} \cdot \mathrm{s}]}\end{array}$ & $\begin{array}{l}\text { Height } \\
{[\mu \mathrm{V}]}\end{array}$ & $\begin{array}{c}\text { Area } \\
{[\%]}\end{array}$ & $\begin{array}{c}\text { Norm. Area } \\
{[\%]}\end{array}$ & $B L$ & $\begin{array}{c}\text { Area/Height } \\
\text { [s] }\end{array}$ \\
\hline - & 0.001 & 0.00 & 0.00 & 0.00 & 0.00 & & \\
\hline 1 & 0.096 & 105.40 & 77.96 & 0.02 & 0.02 & BB & 1.3521 \\
\hline 2 & 0.290 & 763.20 & 96.66 & 0.13 & 0.13 & BB & 7.8955 \\
\hline 3 & 1.398 & 283.20 & 63.58 & 0.05 & 0.05 & BB & 4.4540 \\
\hline 4 & 1.556 & 373.80 & 86.96 & 0.06 & 0.06 & BB & 4.2985 \\
\hline 5 & 1.713 & 253.80 & 66.50 & 0.04 & 0.04 & BB & 3.8167 \\
\hline 6 & 2.021 & 173.80 & 49.99 & 0.03 & 0.03 & BB & 3.4765 \\
\hline 7 & 2.838 & 394.00 & 59.28 & 0.07 & 0.07 & $\mathrm{BB}$ & 6.6464 \\
\hline 8 & 3.008 & 94.40 & 37.10 & 0.02 & 0.02 & BB & 2.5445 \\
\hline 9 & 3.479 & 61.40 & 28.03 & 0.01 & 0.01 & $\mathrm{BB}$ & 2.1904 \\
\hline 10 & 3.658 & 157.20 & 47.50 & 0.03 & 0.03 & BB & 3.3097 \\
\hline 11 & 4.117 & 320.60 & 67.88 & 0.06 & 0.06 & BB & 4.7228 \\
\hline 12 & 4.301 & 343.11 & 75.21 & 0.06 & 0.06 & $\mathrm{BV}$ & 4.5623 \\
\hline 13 & 4.454 & 225.29 & 46.21 & 0.04 & 0.04 & VB & 4.8754 \\
\hline 14 & 4.632 & 153.60 & 44.34 & 0.03 & 0.03 & BB & 3.4645 \\
\hline 15 & 5.246 & 12751.90 & 1099.48 & 2.21 & 2.21 & BV & 11.5981 \\
\hline 16 & 5.929 & 118719.26 & 4397.66 & 20.58 & 20.58 & VV & 26.9960 \\
\hline 17 & 6.100 & 60590.78 & 5202.63 & 10.50 & 10.50 & VV & 11.6462 \\
\hline 18 & 6.207 & 49666.05 & 5721.05 & 8.61 & 8.61 & VB & 8.6813 \\
\hline 19 & 7.707 & 149512.86 & 2656.09 & 25.92 & 25.92 & BV & 56.2907 \\
\hline 20 & 7.835 & 16372.79 & 2018.68 & 2.84 & 2.84 & VV & 8.1106 \\
\hline 21 & 7.985 & 11592.86 & 1205.69 & 2.01 & 2.01 & VV & 9.6151 \\
\hline 22 & 8.129 & 2052.29 & 369.41 & 0.36 & 0.36 & VB & 5.5555 \\
\hline 23 & 8.311 & 249.00 & 58.07 & 0.04 & 0.04 & BB & 4.2876 \\
\hline 24 & 9.097 & 16011.20 & 780.96 & 2.78 & 2.78 & $\mathrm{BB}$ & 20.5018 \\
\hline 25 & 10.223 & 100.40 & 43.85 & 0.02 & 0.02 & BB & 2.2895 \\
\hline 26 & 10.697 & 145.20 & 40.34 & 0.03 & 0.03 & BB & 3.5995 \\
\hline 27 & 11.019 & 115.20 & 48.69 & 0.02 & 0.02 & BB & 2.3659 \\
\hline 28 & 11.551 & 1659.60 & 98.34 & 0.29 & 0.29 & $\mathrm{BB}$ & 16.8754 \\
\hline 29 & 12.365 & 144.40 & 60.46 & 0.03 & 0.03 & BB & 2.3884 \\
\hline 30 & 12.683 & 295.40 & 77.44 & 0.05 & 0.05 & BB & 3.8144 \\
\hline 31 & 12.956 & 249.20 & 53.7 & 0.04 & 0.04 & BB & 338 \\
\hline 32 & 13.122 & 287.20 & 88.48 & 0.05 & 0.05 & BB & 3.2459 \\
\hline 33 & 13.448 & 213.00 & 63.27 & 0.04 & 0.04 & BB & 3.3665 \\
\hline 34 & 13.931 & 490.97 & 83.90 & 0.09 & 0.09 & BV & 5.8521 \\
\hline 35 & 14.091 & 280.23 & 62.98 & 0.05 & 0.05 & VB & 4.4496 \\
\hline 36 & 14.569 & 162.80 & 56.85 & 0.03 & 0.03 & BB & 2.8635 \\
\hline 37 & 15.043 & 204.80 & 61.99 & 0.04 & 0.04 & BB & 3.3039 \\
\hline 38 & 15.764 & 1162.45 & 41.57 & 0.20 & 0.20 & BV & 27.9616 \\
\hline 39 & 15.846 & 170.75 & 56.39 & 0.03 & 0.03 & VB & 3.0278 \\
\hline 40 & 16.009 & 560.79 & 91.08 & 0.10 & 0.10 & BV & 6.1569 \\
\hline 41 & 16.167 & 121.01 & 39.99 & 0.02 & 0.02 & VB & 3.0263 \\
\hline 42 & 16.336 & 178.28 & 51.76 & 0.03 & 0.03 & BV & 3.4446 \\
\hline 43 & 16.489 & 712.72 & 127.55 & 0.12 & 0.12 & VB & 5.5879 \\
\hline 44 & 17.014 & 173.20 & 39.38 & 0.03 & 0.03 & BB & 4.3986 \\
\hline 45 & 17.137 & 117.60 & 43.47 & 0.02 & 0.02 & $\mathrm{BB}$ & 2.7658 \\
\hline
\end{tabular}




\begin{tabular}{|c|c|c|c|c|c|c|c|}
\hline $\begin{array}{c}\text { Peak } \\
\quad \#\end{array}$ & $\begin{array}{l}\text { Time } \\
\text { [min] }\end{array}$ & $\begin{array}{l}\text { Area } \\
{[\mu \mathrm{V} \cdot \mathrm{s}]}\end{array}$ & $\begin{array}{l}\text { Height } \\
{[\mu \mathrm{V}]}\end{array}$ & $\begin{array}{c}\text { Area } \\
{[\%]}\end{array}$ & $\begin{array}{c}\text { Norm. Area } \\
{[\%]}\end{array}$ & $\mathrm{BL}$ & $\begin{array}{c}\text { Area/Height } \\
{[\mathrm{s}]}\end{array}$ \\
\hline 46 & 17.469 & 158.00 & 35.93 & 0.03 & 0.03 & BB & 4.3969 \\
\hline 47 & 17.611 & 119.20 & 41.71 & 0.02 & 0.02 & BB & 2.8579 \\
\hline 48 & 17.777 & 66.00 & 28.18 & 0.01 & 0.01 & BB & 2.3420 \\
\hline 49 & 17.963 & 328.00 & 58.57 & 0.06 & 0.06 & BB & 5.6005 \\
\hline 50 & 18.100 & 84.00 & 43.82 & 0.01 & 0.01 & BB & 1.9171 \\
\hline 51 & 19.178 & 2444.45 & 199.01 & 0.42 & 0.42 & BV & 12.2832 \\
\hline 52 & 19.227 & 687.17 & 180.44 & 0.12 & 0.12 & VV & 3.8082 \\
\hline 53 & 19.315 & 512.12 & 143.45 & 0.09 & 0.09 & VV & 3.5700 \\
\hline 54 & 19.445 & 692.86 & 76.36 & 0.12 & 0.12 & VB & 9.0732 \\
\hline 55 & 20.244 & 86.40 & 41.94 & 0.01 & 0.01 & BB & 2.0599 \\
\hline 56 & 20.373 & 254.80 & 63.37 & 0.04 & 0.04 & BB & 4.0210 \\
\hline 57 & 20.501 & 258.80 & 73.53 & 0.04 & 0.04 & BB & 3.5197 \\
\hline 58 & 20.814 & 71.20 & 35.47 & 0.01 & 0.01 & BB & 2.0074 \\
\hline 59 & 20.999 & 231.39 & 55.13 & 0.04 & 0.04 & BV & 4.1968 \\
\hline 60 & 21.624 & 119898.61 & 3193.61 & 20.78 & 20.78 & VB & 37.5433 \\
\hline 61 & 22.765 & 454.00 & 87.81 & 0.08 & 0.08 & BB & 5.1700 \\
\hline 62 & 22.923 & 358.40 & 85.66 & 0.06 & 0.06 & BB & 4.1838 \\
\hline 63 & 23.078 & 265.80 & 81.61 & 0.05 & 0.05 & BB & 3.2568 \\
\hline 64 & 23.225 & 191.00 & 86.16 & 0.03 & 0.03 & BB & 2.2169 \\
\hline 65 & 24.038 & 234.00 & 75.92 & 0.04 & 0.04 & BB & 3.0822 \\
\hline 66 & 24.198 & 72.80 & 30.95 & 0.01 & 0.01 & BB & 2.3518 \\
\hline 67 & 24.371 & 208.40 & 35.54 & 0.04 & 0.04 & BB & 5.8634 \\
\hline 68 & 24.530 & 226.40 & 47.37 & 0.04 & 0.04 & BB & 4.7789 \\
\hline 69 & 24.850 & 187.80 & 50.53 & 0.03 & 0.03 & $\mathrm{BB}$ & 3.7167 \\
\hline & & & & & & & \\
\hline
\end{tabular}

Warning -- Signal level out-of-range in peak

Missing Component Report

Component Expected Retention (Calibration File) 
Time of Injection: 6/11/2013 2:12:29 PM

Low Point : $-0.41 \mathrm{mV} \quad$ High Point : $8.15 \mathrm{mV}$

Plot Scale: $8.6 \mathrm{mV}$

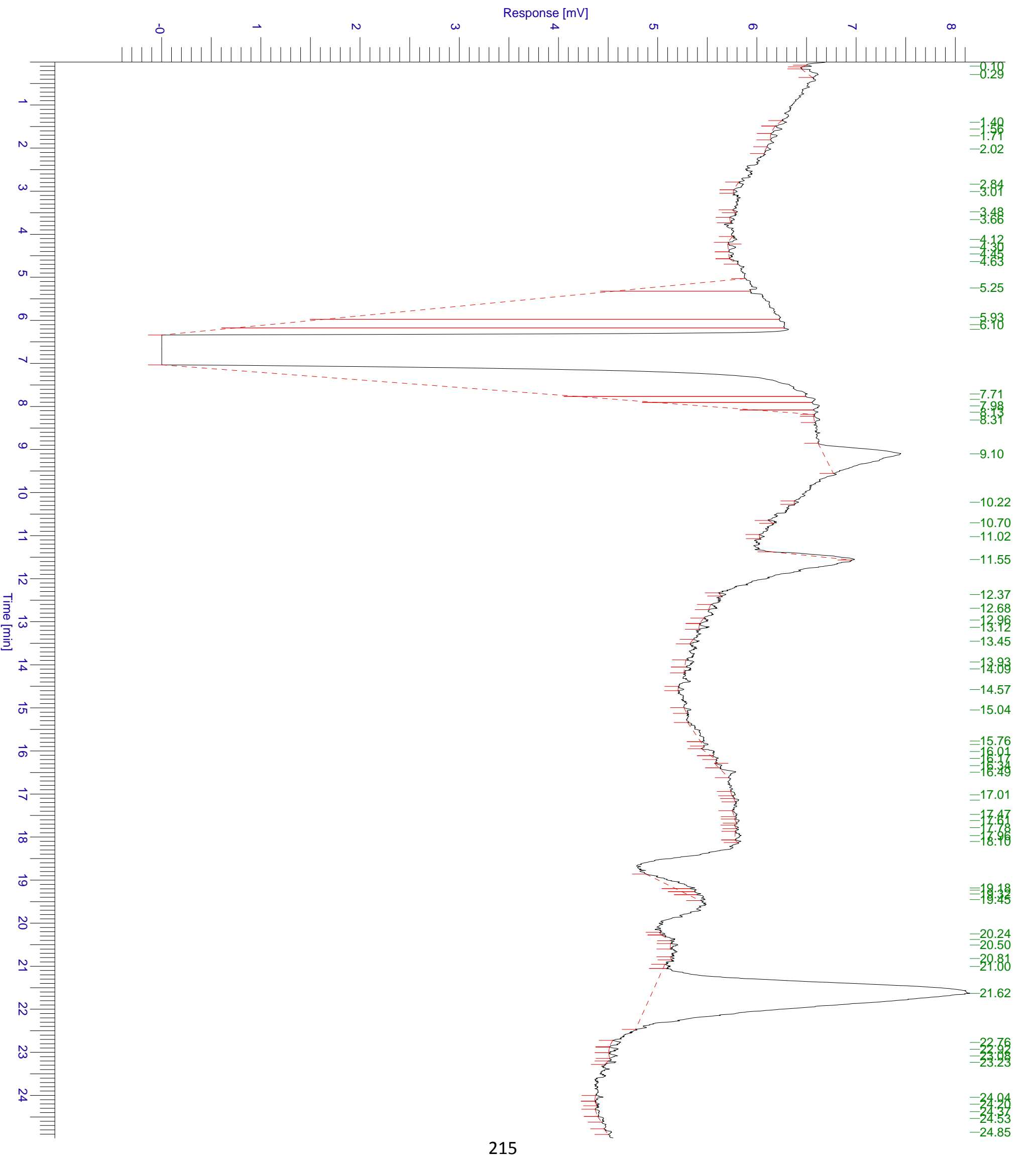




$\begin{array}{llll}\text { Software Version } & : 6.3 .2 .0646 & \text { Date } & : 6 / 21 / 201312: 42: 55 \text { PM } \\ \text { Operator } & : \text { rLuong } & \text { Sample Name } & : \text { St2 } \\ \text { Sample Number } & : \text { O04 } & \text { Study } & : \text { Ethanol } \\ \text { AutoSampler } & : \text { SER200 } & \text { Rack/Vial } & : 1 / 4 \\ \text { Instrument Name } & : \text { HPLC } & \text { Channel } & : \text { A } \\ \text { Instrument Serial \# } & : \text { None } & \text { A/D mV Range }: 1000 \\ \text { Delay Time } & : 0.00 \mathrm{~min} & \text { End Time } & : 24.99 \mathrm{~min} \\ \text { Sampling Rate } & : 2.5000 \mathrm{pts} / \mathrm{s} & & \\ \text { Sample Volume } & : 1.000000 \mathrm{ul} & & \\ \text { Sample Amount } & : 1.0000 & \text { Area Reject } & : 0.000000 \\ \text { Data Acquisition Time } & : 6 / 11 / 20132: 38: 45 \mathrm{PM} & \text { Dilution Factor }: 1.00\end{array}$

Raw Data File : C:IHPLC DatalrobinlEthanol_June 10-2013IEthanol004.raw

Result File : c:Ihplc datalrobinlethanol_june 10-2013lethanol004.rst [Editing in Progress]

Inst Method : C:IHPLC DatalrobinlMethod Robin 87H from C:IHPLC DatalrobinlEthanol_June 10-2013।Ethanol004.raw

Proc Method : C:IHPLC DatalrobinlMethod Robin 87H from c:Ihplc datalrobinlethanol_june 10-2013lethanol004.rst [Editing in Progress] Calib Method : C:IHPLC DatalrobinlMethod Robin 87H from c:Ihplc datalrobinlethanol_june 10-2013lethanol004.rst [Editing in Progress]

Report Format File: C:IHPLC DatalrobinlMethod Robin 87H.rpt

Sequence File : C:IHPLC DatalrobinIValera Sequence 87H-Etanol_June 10_2013.seq

\begin{tabular}{|c|c|c|c|c|c|c|c|}
\hline $\begin{array}{c}\text { Peak } \\
\#\end{array}$ & $\begin{array}{l}\text { Time } \\
\text { [min] }\end{array}$ & $\begin{array}{l}\text { Area } \\
{[\mu \mathrm{V} \cdot \mathrm{s}]}\end{array}$ & $\begin{array}{l}\text { Height } \\
{[\mu \mathrm{V}]}\end{array}$ & $\begin{array}{c}\text { Area } \\
{[\%]}\end{array}$ & $\begin{array}{c}\text { Norm. Area } \\
{[\%]}\end{array}$ & $B L$ & $\begin{array}{c}\text { Area/Height } \\
{[\mathrm{s}]}\end{array}$ \\
\hline- & 0.001 & 0.00 & 0.00 & 0.00 & 0.00 & & \\
\hline 1 & 0.218 & 265.20 & 52.85 & 0.12 & 0.12 & BV & 5.0177 \\
\hline 2 & 0.393 & 379.91 & 46.28 & 0.17 & 0.17 & VV & 8.2083 \\
\hline 3 & 0.524 & 276.69 & 63.17 & 0.13 & 0.13 & VB & 4.3800 \\
\hline 4 & 0.663 & 94.00 & 40.72 & 0.04 & 0.04 & BB & 2.3083 \\
\hline 5 & 0.868 & 274.01 & 59.50 & 0.13 & 0.13 & $\mathrm{BV}$ & 4.6049 \\
\hline 6 & 1.006 & 396.37 & 78.12 & 0.18 & 0.18 & VV & 5.0741 \\
\hline 7 & 1.141 & 192.74 & 68.24 & 0.09 & 0.09 & VB & 2.8243 \\
\hline 8 & 1.307 & 153.30 & 46.22 & 0.07 & 0.07 & BV & 3.3168 \\
\hline 9 & 1.467 & 247.90 & 56.79 & 0.11 & 0.11 & VB & 4.3653 \\
\hline 10 & 1.697 & 324.00 & 57.26 & 0.15 & 0.15 & BB & 5.6589 \\
\hline 11 & 2.275 & 116.00 & 42.06 & 0.05 & 0.05 & BB & 2.7577 \\
\hline 12 & 2.917 & 144.80 & 53.48 & 0.07 & 0.07 & BB & 2.7077 \\
\hline 13 & 3.237 & 260.80 & 49.89 & 0.12 & 0.12 & BB & 5.2276 \\
\hline 14 & 4.353 & 255.40 & 59.94 & 0.12 & 0.12 & BB & 4.2610 \\
\hline 15 & 5.466 & 56.60 & 32.34 & 0.03 & 0.03 & BB & 1.7503 \\
\hline 16 & 5.670 & 159.40 & 51.17 & 0.07 & 0.07 & BB & 3.1150 \\
\hline 17 & 5.803 & 145.20 & 60.18 & 0.07 & 0.07 & BB & 2.4126 \\
\hline 18 & 7.894 & 105493.80 & 299.73 & 48.53 & 48.53 & $\mathrm{BB}$ & 351.9652 \\
\hline 19 & 8.055 & 251.95 & 50.08 & 0.12 & 0.12 & BV & 5.0309 \\
\hline 20 & 8.245 & 380.36 & 56.22 & 0.17 & 0.17 & VV & 6.7657 \\
\hline 21 & 8.512 & 483.69 & 54.49 & 0.22 & 0.22 & VB & 8.8764 \\
\hline 22 & 9.073 & 1786.80 & 175.11 & 0.82 & 0.82 & BB & 10.2036 \\
\hline 23 & 9.819 & 120.80 & 34.74 & 0.06 & 0.06 & BB & 3.4770 \\
\hline 24 & 10.139 & 128.80 & 32.23 & 0.06 & 0.06 & BB & 3.9969 \\
\hline 25 & 10.471 & 121.40 & 42.27 & 0.06 & 0.06 & BB & 2.8718 \\
\hline 26 & 10.678 & 187.60 & 37.49 & 0.09 & 0.09 & BB & 5.0045 \\
\hline 27 & 10.942 & 76.00 & 42.89 & 0.03 & 0.03 & BB & 1.7719 \\
\hline 28 & 11.577 & 3103.60 & 218.51 & 1.43 & 1.43 & BB & 14.2035 \\
\hline 29 & 12.227 & 158.40 & 64.72 & 0.07 & 0.07 & BB & 2.4473 \\
\hline 30 & 12.704 & 97.80 & 51.46 & 0.04 & 0.04 & BB & 1.9003 \\
\hline 31 & 13.027 & 112.00 & 48. & 0.05 & 0.05 & BB & 2.3243 \\
\hline 32 & 13.510 & 126.60 & 76.59 & 0.06 & 0.06 & BB & 1.6530 \\
\hline 33 & 14.650 & 145.00 & 41.90 & 0.07 & 0.07 & BB & 3.4610 \\
\hline 34 & 14.967 & 262.00 & 65.92 & 0.12 & 0.12 & BB & 3.9747 \\
\hline 35 & 15.122 & 298.40 & 65.21 & 0.14 & 0.14 & BB & 4.5763 \\
\hline 36 & 15.445 & 423.40 & 88.99 & 0.19 & 0.19 & BB & 4.7576 \\
\hline 37 & 16.143 & 437.80 & 81.55 & 0.20 & 0.20 & BB & 5.3682 \\
\hline 38 & 16.253 & 132.00 & 49.32 & 0.06 & 0.06 & BB & 2.6762 \\
\hline 39 & 16.405 & 339.60 & 83.36 & 0.16 & 0.16 & BB & 4.0738 \\
\hline 40 & 16.555 & 260.00 & 73.69 & 0.12 & 0.12 & BB & 3.5283 \\
\hline 41 & 17.372 & 378.40 & 67.38 & 0.17 & 0.17 & $\mathrm{BB}$ & 5.6159 \\
\hline 42 & 17.692 & 60.80 & 34.53 & 0.03 & 0.03 & BB & 1.7607 \\
\hline 43 & 18.843 & 199.00 & 39.20 & 0.09 & 0.09 & BB & 5.0771 \\
\hline 44 & 19.483 & 5002.68 & 136.42 & 2.30 & 2.30 & BV & 36.6712 \\
\hline 45 & 19.634 & 220.92 & 52.61 & 0.10 & 0.10 & VB & $4.139 \overline{6}$ \\
\hline
\end{tabular}


6/21/2013 12:42:55 PM Result: c:Ihplc datalrobinlethanol june 10-2013lethanol004.rst

\begin{tabular}{|c|c|c|c|c|c|c|c|}
\hline $\begin{array}{c}\text { Peak } \\
\text { \# }\end{array}$ & $\begin{array}{l}\text { Time } \\
\text { [min] }\end{array}$ & $\begin{array}{l}\text { Area } \\
{[\mu \mathrm{V} \cdot \mathrm{s}]}\end{array}$ & $\begin{array}{l}\text { Height } \\
{[\mu \mathrm{V}]}\end{array}$ & $\begin{array}{c}\text { Area } \\
{[\%]}\end{array}$ & $\begin{array}{c}\text { Norm. Area } \\
{[\%]}\end{array}$ & $B L$ & $\begin{array}{c}\text { Area/Height } \\
{[\mathrm{s}]}\end{array}$ \\
\hline 46 & 20.779 & 3334.96 & 107.42 & 1.53 & 1.53 & BV & 31.0455 \\
\hline 47 & 20.913 & 370.24 & 69.21 & 0.17 & 0.17 & VB & 5.3493 \\
\hline 48 & 21.601 & 63495.33 & 4061.43 & 29.21 & 29.21 & BV & 15.6337 \\
\hline 49 & 21.649 & 24182.87 & 3580.62 & 11.12 & 11.12 & VB & 6.7538 \\
\hline 50 & 22.992 & 121.20 & 48.98 & 0.06 & 0.06 & BB & 2.4743 \\
\hline 51 & 23.149 & 127.40 & 52.48 & 0.06 & 0.06 & BB & 2.4274 \\
\hline 52 & 24.005 & 423.40 & 76.11 & 0.19 & 0.19 & BB & 5.5630 \\
\hline 53 & 24.286 & 360.43 & 83.51 & 0.17 & 0.17 & BV & 4.3159 \\
\hline 54 & 24.433 & 339.97 & 77.04 & 0.16 & 0.16 & VB & 4.4128 \\
\hline \multirow[t]{2}{*}{55} & 24.755 & 96.40 & 62.25 & 0.04 & 0.04 & $\mathrm{BB}$ & 1.5486 \\
\hline & & & & & 100.00 & & \\
\hline
\end{tabular}

Missing Component Report

Component Expected Retention (Calibration File)

0.001 

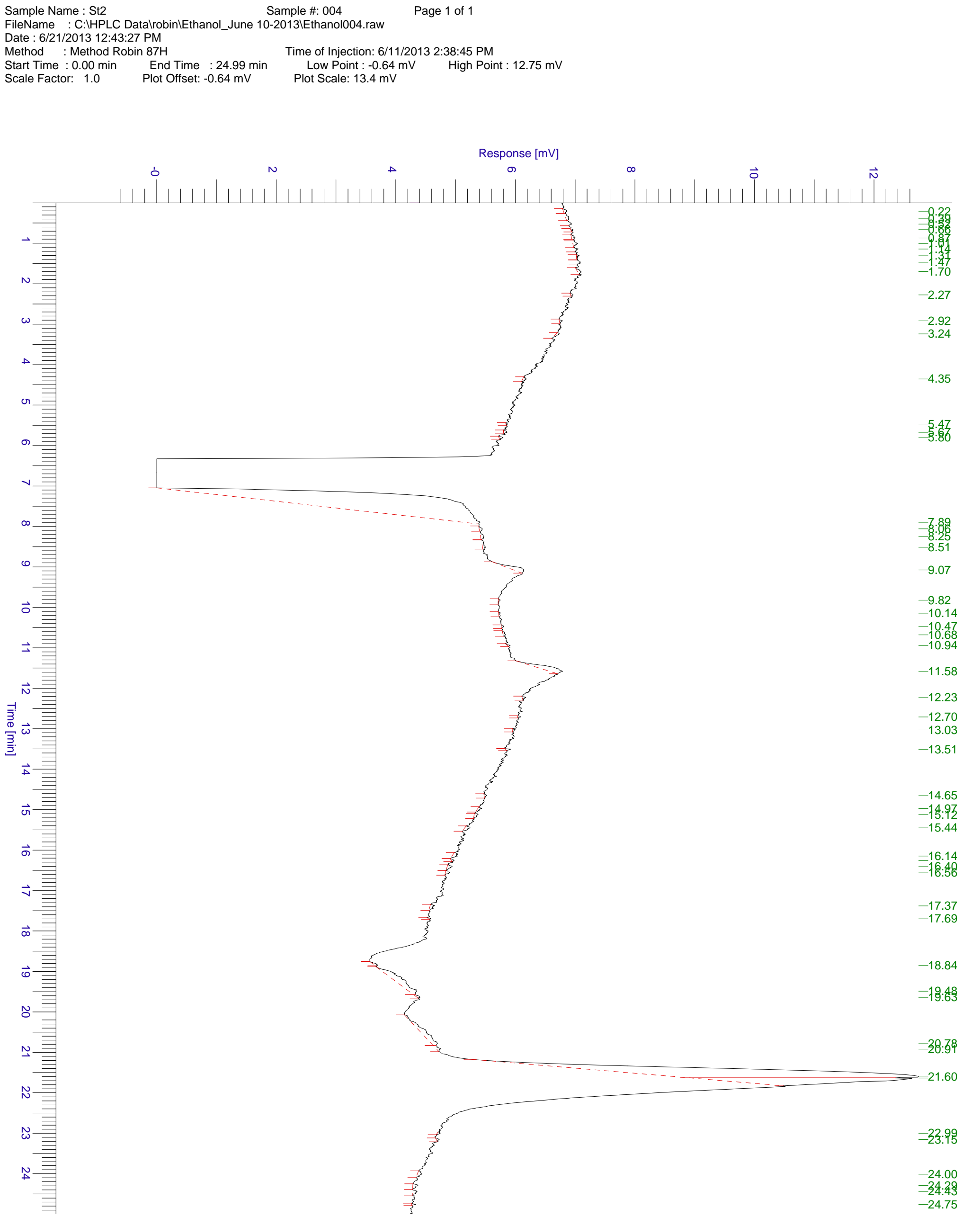


\begin{tabular}{|c|c|c|c|}
\hline Software Version & 6.3.2.0646 & Date & : 6/21/2013 12:44:19 PM \\
\hline Operator & rLuong & Sample Name & : St3 \\
\hline Sample Number & 005 & Study & Ethanol \\
\hline AutoSampler & SER200 & Rack/Vial & $1 / 5$ \\
\hline Instrument Name & HPLC & Channel & $: A$ \\
\hline Instrument Serial \# & None & A/D mV Range & $: 1000$ \\
\hline Delay Time & $0.00 \mathrm{~min}$ & End Time & $24.99 \min$ \\
\hline Sampling Rate & $2.5000 \mathrm{pts} / \mathrm{s}$ & & \\
\hline Sample Volume & $1.000000 \mathrm{ul}$ & & \\
\hline Sample Amount & 1.0000 & Area Reject & $: 0.000000$ \\
\hline Data Acquisition Time & 6/11/2013 3:05:01 PM & $\begin{array}{l}\text { Dilution Factor } \\
\text { Cycle }\end{array}$ & $\begin{array}{l}1.00 \\
: 5\end{array}$ \\
\hline
\end{tabular}

Raw Data File : C:IHPLC DatalrobinlEthanol_June 10-2013lEthanol005.raw

Result File : c:Ihplc datalrobinlethanol_june 10-2013lethanol005.rst [Editing in Progress]

Inst Method : C:IHPLC DatalrobinlMethod Robin 87H from C:IHPLC DatalrobinlEthanol_June 10-2013।Ethanol005.raw

Proc Method : C:IHPLC DatalrobinlMethod Robin 87H from c:Ihplc datalrobinlethanol_june 10-2013lethanol005.rst [Editing in Progress] Calib Method : C:IHPLC DatalrobinlMethod Robin 87H from c:Ihplc datalrobinlethanol_june 10-2013lethanol005.rst [Editing in Progress]

Report Format File: C:IHPLC DatalrobinlMethod Robin 87H.rpt

Sequence File : C:IHPLC DatalrobinIValera Sequence 87H-Etanol_June 10_2013.seq

\begin{tabular}{|c|c|c|c|c|c|c|c|}
\hline $\begin{array}{c}\text { Peak } \\
\#\end{array}$ & $\begin{array}{l}\text { Time } \\
\text { [min] }\end{array}$ & $\begin{array}{l}\text { Area } \\
{[\mu \mathrm{V} \cdot \mathrm{s}]}\end{array}$ & $\begin{array}{c}\text { Height } \\
{[\mu \mathrm{V}]}\end{array}$ & $\begin{array}{c}\text { Area } \\
{[\%]}\end{array}$ & $\begin{array}{c}\text { Norm. Area } \\
{[\%]}\end{array}$ & $\mathrm{BL}$ & $\begin{array}{c}\text { Area/Height } \\
\text { [s] }\end{array}$ \\
\hline- & 0.001 & 0.00 & 0.00 & 0.00 & 0.00 & & \\
\hline 1 & 0.135 & 199.60 & 52.14 & 0.02 & 0.02 & BB & 3.8285 \\
\hline 2 & 0.270 & 341.20 & 84.41 & 0.03 & 0.03 & $\mathrm{BB}$ & 4.0422 \\
\hline 3 & 0.939 & 500.00 & 78.52 & 0.04 & 0.04 & BB & 6.3674 \\
\hline 4 & 1.071 & 139.40 & 41.89 & 0.01 & 0.01 & BB & 3.3279 \\
\hline 5 & 1.235 & 287.58 & 66.39 & 0.02 & 0.02 & BV & 4.3316 \\
\hline 6 & 1.380 & 212.67 & 56.18 & 0.02 & 0.02 & VV & 3.7853 \\
\hline 7 & 1.547 & 273.35 & 78.14 & 0.02 & 0.02 & VB & 3.4979 \\
\hline 8 & 2.009 & 151.00 & 33.03 & 0.01 & 0.01 & BB & 4.5717 \\
\hline 9 & 2.210 & 192.80 & 42.71 & 0.02 & 0.02 & BB & 4.5143 \\
\hline 10 & 2.508 & 70.20 & 35.60 & 0.01 & 0.01 & BB & 1.9717 \\
\hline 11 & 2.668 & 163.43 & 43.07 & 0.01 & 0.01 & BV & 3.7949 \\
\hline 12 & 3.473 & 167.73 & 30.32 & 0.01 & 0.01 & VB & 5.5329 \\
\hline 13 & 4.290 & 1020.80 & 37.53 & 0.09 & 0.09 & BB & 27.1990 \\
\hline 14 & 4.491 & 320.63 & 49.63 & 0.03 & 0.03 & BV & 6.4599 \\
\hline 15 & 5.232 & 875.28 & 36.87 & 0.08 & 0.08 & VB & 23.7368 \\
\hline 16 & 6.047 & 217.00 & 66.18 & 0.02 & 0.02 & BB & 3.2791 \\
\hline 17 & 7.800 & 200857.73 & 2178.80 & 17.39 & 17.39 & BV & 92.1874 \\
\hline 18 & 7.981 & 9856.67 & 871.85 & 0.85 & 0.85 & VB & 11.3055 \\
\hline 19 & 9.089 & 19510.40 & 820.18 & 1.69 & 1.69 & $\mathrm{BE}$ & 23.7880 \\
\hline 20 & 9.742 & 298.00 & 66.19 & 0.03 & 0.03 & EB & 4.5024 \\
\hline 21 & 10.607 & 235.18 & 30.49 & 0.02 & 0.02 & $\mathrm{BV}$ & 7.7127 \\
\hline 22 & 10.750 & 207.42 & 28.95 & 0.02 & 0.02 & VB & 7.1646 \\
\hline 23 & 11.064 & 249.20 & 45.34 & 0.02 & 0.02 & BB & 5.4965 \\
\hline 24 & 11.198 & 213.58 & 58.91 & 0.02 & 0.02 & BV & 3.6255 \\
\hline 25 & 11.551 & 30927.02 & 1121.09 & 2.68 & 2.68 & VB & 27.5866 \\
\hline 26 & 12.537 & 121.60 & 16.50 & 0.01 & 0.01 & BB & 7.3681 \\
\hline 27 & 12.809 & 231.60 & 56.10 & 0.02 & 0.02 & $\mathrm{BB}$ & 4.1281 \\
\hline 28 & 13.274 & 68.80 & 24.01 & 0.01 & 0.01 & BB & 2.8649 \\
\hline 29 & 13.479 & 189.20 & 30.31 & 0.02 & 0.02 & $\mathrm{BB}$ & 6.2430 \\
\hline 30 & 15.291 & 1760.00 & 40.24 & 0.15 & 0.15 & BB & 43.7350 \\
\hline 31 & 16.318 & 392.60 & 68.45 & 0.03 & 0.03 & BB & 5.7356 \\
\hline 32 & 17.313 & 6140.96 & 199.78 & 0.53 & 0.53 & BV & 30.7381 \\
\hline 33 & 17.558 & 1993.99 & 156.58 & 0.17 & 0.17 & VV & 12.7346 \\
\hline 34 & 17.688 & 559.14 & 71.22 & 0.05 & 0.05 & VV & 7.8515 \\
\hline 35 & 17.806 & 407.90 & 91.38 & 0.04 & 0.04 & VB & 4.4636 \\
\hline 36 & 17.931 & 212.81 & 83.59 & 0.02 & 0.02 & BV & 2.5459 \\
\hline 37 & 18.051 & 326.02 & 105.58 & 0.03 & 0.03 & VV & 3.0880 \\
\hline 38 & 18.175 & 712.41 & 129.04 & 0.06 & 0.06 & VV & 5.5208 \\
\hline 39 & 18.299 & 553.96 & 88.58 & 0.05 & 0.05 & VB & 6.2536 \\
\hline 40 & 18.949 & 3728.80 & 475.81 & 0.32 & 0.32 & BB & 7.8368 \\
\hline 41 & 19.255 & 4649.19 & 539.13 & 0.40 & 0.40 & BV & 8.6236 \\
\hline 42 & 19.282 & 1005.91 & 520.80 & 0.09 & 0.09 & VV & 1.9315 \\
\hline 43 & 19.402 & 3704.65 & 437.52 & 0.32 & 0.32 & VV & 8.4674 \\
\hline 44 & 19.528 & 1864.39 & 272.17 & 0.16 & 0.16 & VV & 6.8500 \\
\hline 45 & 19.600 & 419.06 & 97.86 & 0.04 & 0.04 & VB & 4.2823 \\
\hline
\end{tabular}


6/21/2013 12:44:19 PM Result: c:Ihplc datalrobinlethanol_june 10-2013lethanol005.rst

\begin{tabular}{|c|c|c|c|c|c|c|c|}
\hline $\begin{array}{c}\text { Peak } \\
\quad \#\end{array}$ & $\begin{array}{l}\text { Time } \\
\text { [min] }\end{array}$ & $\begin{array}{l}\text { Area } \\
{[\mu \mathrm{V} \cdot \mathrm{s}]}\end{array}$ & $\begin{array}{l}\text { Height } \\
{[\mu \mathrm{V}]}\end{array}$ & $\begin{array}{c}\text { Area } \\
{[\%]}\end{array}$ & $\begin{array}{c}\text { Norm. Area } \\
{[\%]}\end{array}$ & $\mathrm{BL}$ & $\begin{array}{c}\text { Area/Height } \\
\text { [s] }\end{array}$ \\
\hline 46 & 19.712 & 170.82 & 85.85 & 0.01 & 0.01 & BV & 1.9898 \\
\hline 47 & 19.752 & 245.58 & 94.28 & 0.02 & 0.02 & VB & 2.6049 \\
\hline 48 & 20.010 & 272.00 & 119.21 & 0.02 & 0.02 & BB & 2.2818 \\
\hline 49 & 20.180 & 630.82 & 189.77 & 0.05 & 0.05 & BV & 3.3241 \\
\hline 50 & 20.216 & 500.52 & 304.46 & 0.04 & 0.04 & VV & 1.6439 \\
\hline 51 & 20.240 & 458.89 & 329.56 & 0.04 & 0.04 & VV & 1.3924 \\
\hline 52 & 20.436 & 7425.84 & 932.25 & 0.64 & 0.64 & VV & 7.9655 \\
\hline 53 & 20.481 & 3359.15 & 1035.89 & 0.29 & 0.29 & VV & 3.2428 \\
\hline 54 & 20.565 & 4839.36 & 1266.97 & 0.42 & 0.42 & VV & 3.8196 \\
\hline 55 & 20.599 & 2521.23 & 1299.93 & 0.22 & 0.22 & VV & 1.9395 \\
\hline 56 & 20.690 & 9005.62 & 1479.36 & 0.78 & 0.78 & VV & 6.0875 \\
\hline 57 & 20.755 & 5469.03 & 1612.08 & 0.47 & 0.47 & VV & 3.3925 \\
\hline 58 & 20.892 & 15159.40 & 2018.81 & 1.31 & 1.31 & VV & 7.5091 \\
\hline 59 & 21.019 & 16420.82 & 2387.65 & 1.42 & 1.42 & VV & 6.8774 \\
\hline 60 & 21.615 & 788525.31 & 17991.56 & 68.28 & 68.28 & VB & 43.8275 \\
\hline 61 & 22.971 & 2414.60 & 471.54 & 0.21 & 0.21 & $\mathrm{BB}$ & 5.1206 \\
\hline 62 & 23.700 & 188.80 & 62.96 & 0.02 & 0.02 & $\mathrm{BB}$ & 2.9989 \\
\hline 63 & 24.287 & 328.00 & 302.98 & 0.03 & 0.03 & BB & 1.0826 \\
\hline 64 & 24.521 & 168.00 & 51.70 & 0.01 & 0.01 & $\mathrm{BB}$ & 3.2496 \\
\hline \multirow[t]{2}{*}{65} & 24.889 & 213.60 & 42.81 & 0.02 & 0.02 & $\mathrm{BB}$ & 4.9900 \\
\hline & & 848.27 & 1638.67 & & 100.00 & & \\
\hline
\end{tabular}

Missing Component Report

Component Expected Retention (Calibration File) 
Time of Injection: 6/11/2013 3:05:01 PM

Low Point : $-1.74 \mathrm{mV}$

Plot Scale: $36.5 \mathrm{mV}$

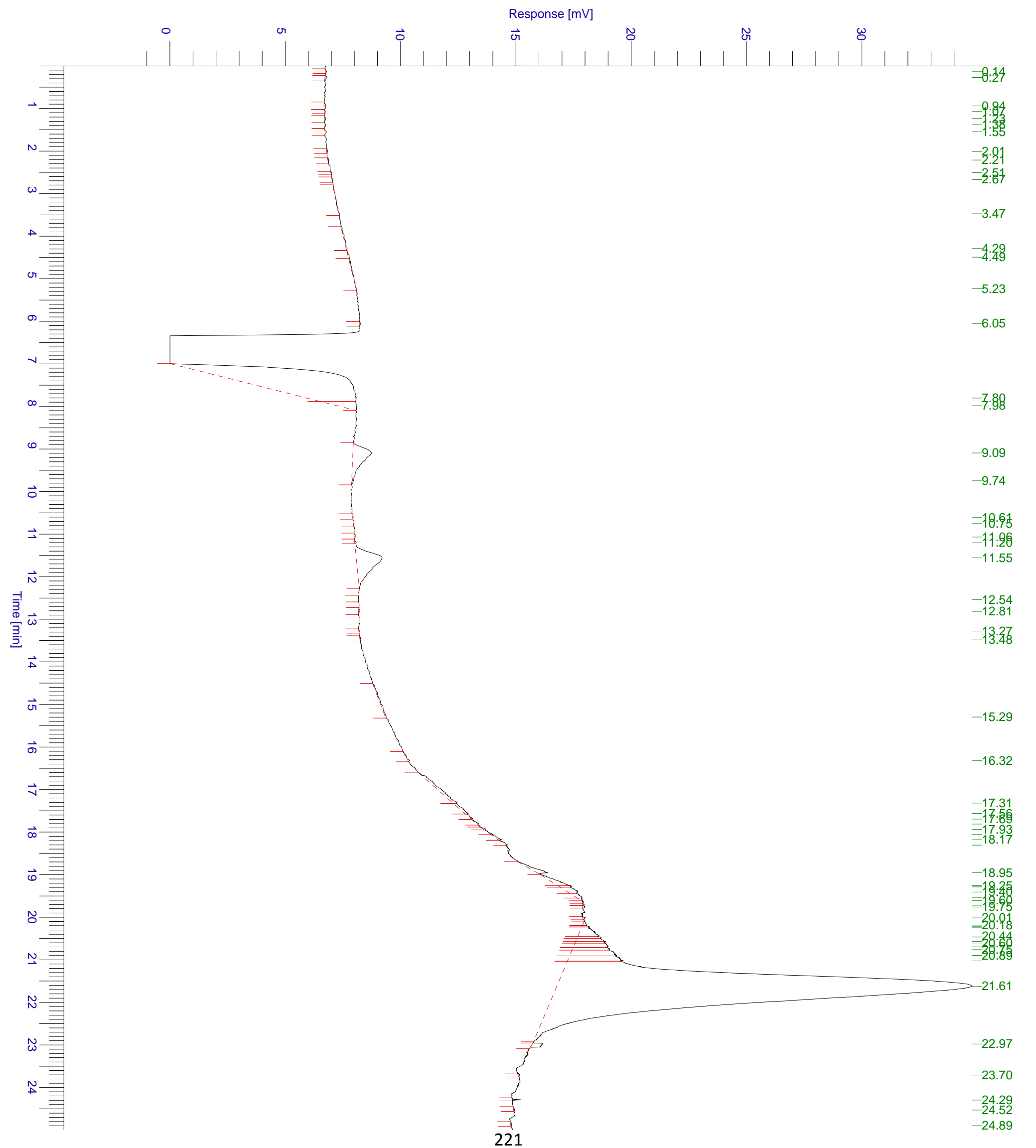




\begin{tabular}{|c|c|c|c|}
\hline Software Version & 6.3.2.0646 & Date & : 6/21/2013 12:46:16 PM \\
\hline Operator & rLuong & Sample Name & : St4 \\
\hline Sample Number & 006 & Study & Ethanol \\
\hline AutoSampler & SER200 & Rack/Vial & $1 / 6$ \\
\hline Instrument Name & HPLC & Channel & A \\
\hline Instrument Serial \# & None & A/D mV Range & $: 1000$ \\
\hline Delay Time & $0.00 \mathrm{~min}$ & End Time & $24.99 \min$ \\
\hline Sampling Rate & $2.5000 \mathrm{pts} / \mathrm{s}$ & & \\
\hline Sample Volume & $1.000000 \mathrm{ul}$ & & \\
\hline Sample Amount & 1.0000 & Area Reject & 0.000000 \\
\hline Data Acquisition Time & 6/11/2013 3:31:17 PM & $\begin{array}{l}\text { Dilution Factor } \\
\text { Cycle }\end{array}$ & $\begin{array}{l}: 1.00 \\
: 6\end{array}$ \\
\hline
\end{tabular}

Raw Data File : C:IHPLC DatalrobinlEthanol_June 10-2013lEthanol006.raw

Result File : c:Ihplc datalrobinlethanol_june 10-2013lethanol006.rst [Editing in Progress]

Inst Method : C:IHPLC DatalrobinlMethod Robin 87H from C:IHPLC DatalrobinlEthanol_June 10-2013।Ethanol006.raw

Proc Method : C:IHPLC DatalrobinlMethod Robin 87H from c:Ihplc datalrobinlethanol_june 10-2013lethanol006.rst [Editing in Progress] Calib Method : C:IHPLC DatalrobinlMethod Robin 87H from c:Ihplc datalrobinlethanol_june 10-2013lethanol006.rst [Editing in Progress]

Report Format File: C:IHPLC DatalrobinlMethod Robin 87H.rpt

Sequence File : C:IHPLC DatalrobinIValera Sequence 87H-Etanol_June 10_2013.seq

\begin{tabular}{|c|c|c|c|c|c|c|c|}
\hline $\begin{array}{c}\text { Peak } \\
\#\end{array}$ & $\begin{array}{l}\text { Time } \\
\text { [min] }\end{array}$ & $\begin{array}{l}\text { Area } \\
{[\mu \mathrm{V} \cdot \mathrm{s}]}\end{array}$ & $\begin{array}{l}\text { Height } \\
{[\mu \mathrm{V}]}\end{array}$ & $\begin{array}{c}\text { Area } \\
{[\%]}\end{array}$ & $\begin{array}{c}\text { Norm. Area } \\
{[\%]}\end{array}$ & $B L$ & $\begin{array}{c}\text { Area/Height } \\
{[\mathrm{s}]}\end{array}$ \\
\hline- & 0.001 & 0.00 & 0.00 & 0.00 & 0.00 & & \\
\hline 1 & 0.290 & 53.20 & 46.18 & 0.01 & 0.01 & BB & 1.1519 \\
\hline 2 & 0.337 & 118.41 & 86.08 & 0.03 & 0.03 & $\mathrm{BV}$ & 1.3755 \\
\hline 3 & 0.420 & 249.88 & 87.33 & 0.06 & 0.06 & VV & 2.8613 \\
\hline 4 & 0.546 & 771.17 & 133.32 & 0.20 & 0.20 & VV & 5.7843 \\
\hline 5 & 0.595 & 126.55 & 70.64 & 0.03 & 0.03 & VB & 1.7913 \\
\hline 6 & 0.729 & 1068.72 & 228.59 & 0.27 & 0.27 & BV & 4.6753 \\
\hline 7 & 0.863 & 2000.18 & 306.85 & 0.51 & 0.51 & VV & 6.5185 \\
\hline 8 & 0.994 & 2557.84 & 314.52 & 0.65 & 0.65 & VV & 8.1325 \\
\hline 9 & 1.055 & 795.29 & 264.31 & 0.20 & 0.20 & VV & 3.0089 \\
\hline 10 & 1.129 & 589.44 & 212.24 & 0.15 & 0.15 & VV & 2.7772 \\
\hline 11 & 1.208 & 600.57 & 148.18 & 0.15 & 0.15 & VV & 4.0529 \\
\hline 12 & 1.252 & 284.72 & 160.11 & 0.07 & 0.07 & VV & 1.7783 \\
\hline 13 & 1.291 & 318.05 & 113.65 & 0.08 & 0.08 & VB & 2.7984 \\
\hline 14 & 1.411 & 135.20 & 56.31 & 0.03 & 0.03 & BB & 2.4008 \\
\hline 15 & 1.468 & 189.20 & 93.77 & 0.05 & 0.05 & BB & 2.0178 \\
\hline 16 & 1.516 & 81.80 & 65.85 & 0.02 & 0.02 & BB & 1.2422 \\
\hline 17 & 1.607 & 138.40 & 107.99 & 0.04 & 0.04 & BB & 1.2816 \\
\hline 18 & 1.649 & 104.40 & 69.88 & 0.03 & 0.03 & $\mathrm{BB}$ & 1.4940 \\
\hline 19 & 1.781 & 157.04 & 114.72 & 0.04 & 0.04 & BV & 1.3688 \\
\hline 20 & 1.852 & 441.55 & 173.21 & 0.11 & 0.11 & VV & 2.5492 \\
\hline 21 & 1.947 & 965.51 & 196.41 & 0.25 & 0.25 & VB & 4.9157 \\
\hline 22 & 2.130 & 355.23 & 116.06 & 0.09 & 0.09 & BV & 3.0608 \\
\hline 23 & 2.168 & 208.77 & 98.78 & 0.05 & 0.05 & VB & 2.1136 \\
\hline 24 & 2.265 & 168.80 & 112.14 & 0.04 & 0.04 & BB & 1.5052 \\
\hline 25 & 2.982 & 143.92 & 60.54 & 0.04 & 0.04 & BV & 2.3774 \\
\hline 26 & 3.055 & 193.88 & 106.47 & 0.05 & 0.05 & VB & 1.8210 \\
\hline 27 & 3.213 & 58.00 & 52.57 & 0.01 & 0.01 & BB & 1.1032 \\
\hline 28 & 3.248 & 96.60 & 70.71 & 0.02 & 0.02 & BB & 1.3661 \\
\hline 29 & 3.384 & 250.40 & 101.64 & 0.06 & 0.06 & BB & 2.4635 \\
\hline 30 & 3.434 & 46.00 & 45.53 & 0.01 & 0.01 & BB & 1.0102 \\
\hline 31 & 3.535 & 139.60 & 153.21 & 0.04 & 0.04 & BB & 112 \\
\hline 32 & 4.534 & 168.20 & 55.55 & 0.04 & 0.04 & BB & 3.0281 \\
\hline 33 & 4.593 & 91.60 & 91.59 & 0.02 & 0.02 & BB & 1.0001 \\
\hline 34 & 4.953 & 756.00 & 248.92 & 0.19 & 0.19 & BB & 3.0371 \\
\hline 35 & 5.158 & 337.60 & 113.43 & 0.09 & 0.09 & BB & 2.9764 \\
\hline 36 & 5.298 & 43.20 & 57.48 & 0.01 & 0.01 & BB & 0.7515 \\
\hline 37 & 5.629 & 989.00 & 211.40 & 0.25 & 0.25 & BB & 4.6784 \\
\hline 38 & 5.749 & 59.80 & 72.91 & 0.02 & 0.02 & BB & 0.8202 \\
\hline 39 & 6.180 & 1777.60 & 56.88 & 0.45 & 0.45 & BB & 31.2493 \\
\hline 40 & 7.352 & 91975.08 & 4281.19 & 23.41 & 23.41 & $\mathrm{BV}$ & 21.4835 \\
\hline 41 & 7.423 & 12884.63 & 3760.88 & 3.28 & 3.28 & VV & 3.4260 \\
\hline 42 & 7.643 & 33411.07 & 2368.09 & 8.50 & 8.50 & VV & 14.1089 \\
\hline 43 & 7.717 & 11156.70 & 1800.97 & 2.84 & 2.84 & VV & 6.1948 \\
\hline 44 & 7.875 & 1492.90 & 490.49 & 0.38 & 0.38 & VB & 3.0437 \\
\hline 45 & 8.046 & 544.80 & 116.69 & 0.14 & 0.14 & BB & 4.6687 \\
\hline
\end{tabular}




\begin{tabular}{|c|c|c|c|c|c|c|c|}
\hline $\begin{array}{c}\text { Peak } \\
\quad \#\end{array}$ & $\begin{array}{l}\text { Time } \\
\text { [min] }\end{array}$ & $\begin{array}{l}\text { Area } \\
{[\mu \mathrm{V} \cdot \mathrm{s}]}\end{array}$ & $\begin{array}{l}\text { Height } \\
{[\mu \mathrm{V}]}\end{array}$ & $\begin{array}{c}\text { Area } \\
{[\%]}\end{array}$ & $\begin{array}{c}\text { Norm. Area } \\
{[\%]}\end{array}$ & $B L$ & $\begin{array}{c}\text { Area/Height } \\
\text { [s] }\end{array}$ \\
\hline 46 & 8.339 & 317.40 & 29.38 & 0.08 & 0.08 & BB & 10.8045 \\
\hline 47 & 9.011 & 3317.60 & 360.10 & 0.84 & 0.84 & BB & 9.2131 \\
\hline 48 & 9.237 & 615.60 & 259.20 & 0.16 & 0.16 & BB & 2.3750 \\
\hline 49 & 10.469 & 153.00 & 49.15 & 0.04 & 0.04 & BB & 3.1129 \\
\hline 50 & 10.796 & 371.20 & 62.28 & 0.09 & 0.09 & $B B$ & 5.9600 \\
\hline 51 & 11.546 & 4693.40 & 327.56 & 1.19 & 1.19 & BB & 14.3282 \\
\hline 52 & 12.263 & 252.80 & 56.53 & 0.06 & 0.06 & $\mathrm{BB}$ & 4.4722 \\
\hline 53 & 12.442 & 498.20 & 78.52 & 0.13 & 0.13 & BB & 6.3447 \\
\hline 54 & 12.803 & 50.06 & 50.56 & 0.01 & 0.01 & BV & 0.9901 \\
\hline 55 & 12.835 & 92.53 & 78.28 & 0.02 & 0.02 & VV & 1.1821 \\
\hline 56 & 12.860 & 216.41 & 82.58 & 0.06 & 0.06 & VV & 2.6207 \\
\hline 57 & 12.954 & 167.01 & 62.44 & 0.04 & 0.04 & VB & 2.6747 \\
\hline 58 & 13.070 & 158.00 & 76.87 & 0.04 & 0.04 & BB & 2.0554 \\
\hline 59 & 13.200 & 219.20 & 76.41 & 0.06 & 0.06 & BB & 2.8686 \\
\hline 60 & 13.282 & 50.00 & 53.91 & 0.01 & 0.01 & BB & 0.9274 \\
\hline 61 & 13.550 & 503.20 & 111.12 & 0.13 & 0.13 & BB & 4.5286 \\
\hline 62 & 13.673 & 325.20 & 87.19 & 0.08 & 0.08 & $B B$ & 3.7300 \\
\hline 63 & 15.093 & 630.80 & 123.81 & 0.16 & 0.16 & BB & 5.0950 \\
\hline 64 & 15.750 & 180.00 & 59.31 & 0.05 & 0.05 & BB & 3.0350 \\
\hline 65 & 16.417 & 201.80 & 42.68 & 0.05 & 0.05 & $\mathrm{BB}$ & 4.7282 \\
\hline 66 & 16.551 & 35.00 & 38.72 & 0.01 & 0.01 & BB & 0.9039 \\
\hline 67 & 17.030 & 1664.40 & 72.52 & 0.42 & 0.42 & BB & 22.9493 \\
\hline 68 & 18.008 & 9272.62 & 794.42 & 2.36 & 2.36 & BV & 11.6722 \\
\hline 69 & 18.180 & 29641.78 & 1203.30 & 7.54 & 7.54 & VB & 24.6337 \\
\hline \multirow[t]{2}{*}{70} & 21.593 & 170177.60 & 9402.00 & 43.31 & 43.31 & BB & 18.1001 \\
\hline & & & & & 100.00 & & \\
\hline
\end{tabular}

Warning -- Signal level out-of-range in peak

Missing Component Report

Component Expected Retention (Calibration File)

standards

0.001 


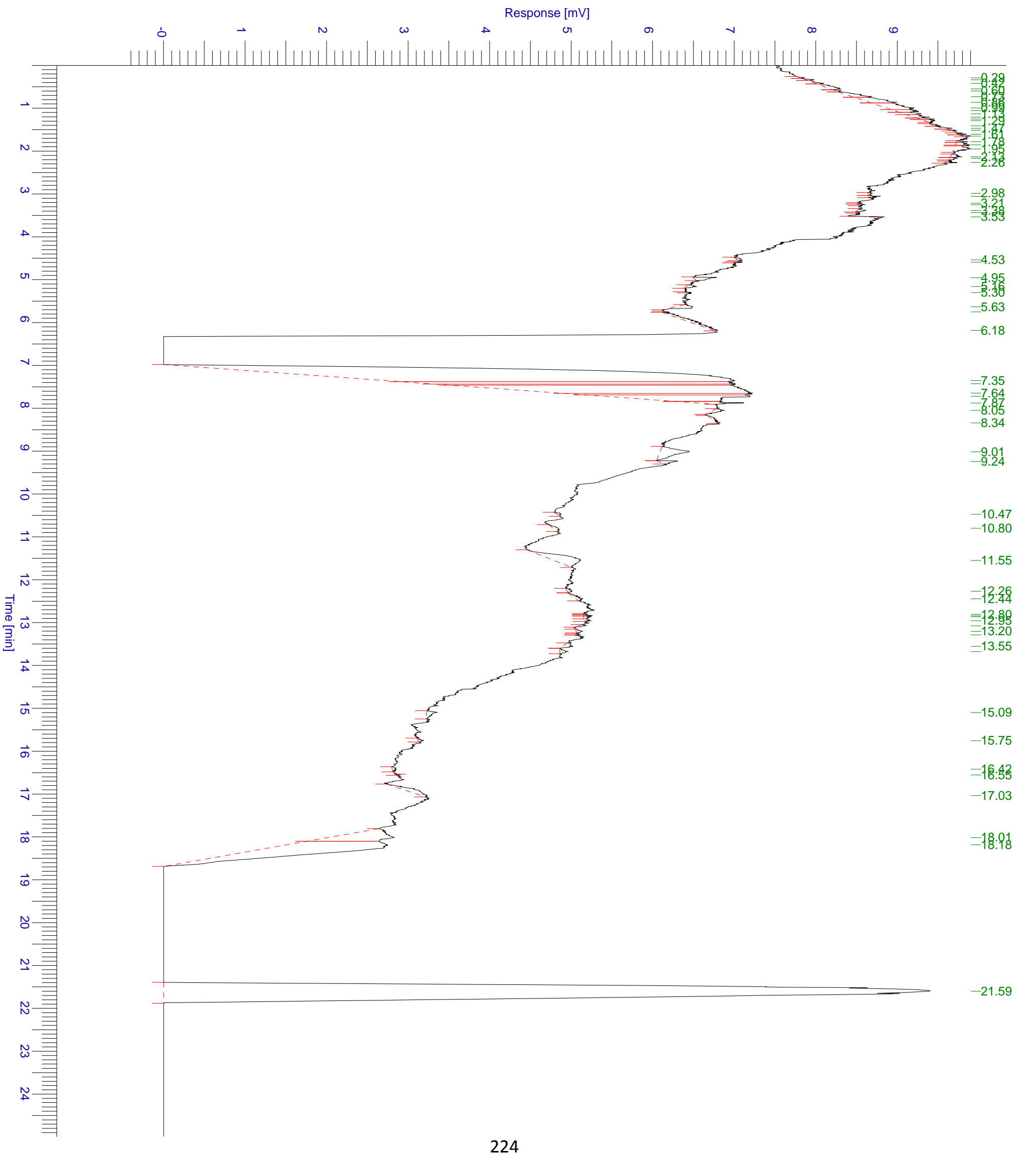




$\begin{array}{llll}\text { Software Version } & : 6.3 .2 .0646 & \text { Date } & : 6 / 21 / 2013 \text { 12:47:01 PM } \\ \text { Operator } & : \text { rLuong } & \text { Sample Name } & : \text { St5 } \\ \text { Sample Number } & : \text { O07 } & \text { Study } & : \text { Ethanol } \\ \text { AutoSampler } & : \text { SER200 } & \text { Rack/Vial } & : 1 / 7 \\ \text { Instrument Name } & : \text { HPLC } & \text { Channel } & : \text { A } \\ \text { Instrument Serial \# } & : \text { None } & \text { A/D mV Range }: 1000 \\ \text { Delay Time } & : 0.00 \mathrm{~min} & \text { End Time } & : 24.99 \mathrm{~min} \\ \text { Sampling Rate } & : 2.5000 \mathrm{pts} / \mathrm{s} & & \\ \text { Sample Volume } & : 1.000000 \mathrm{ul} & & \\ \text { Sample Amount } & : 1.0000 & \text { Area Reject } & : 0.000000 \\ \text { Data Acquisition Time } & : 6 / 11 / 2013 \text { 3:57:33 PM } & \text { Dilution Factor }: 1.00\end{array}$

Raw Data File : C:IHPLC DatalrobinlEthanol_June 10-2013IEthanol007.raw

Result File : c:Ihplc datalrobinlethanol_june 10-2013lethanol007.rst [Editing in Progress]

Inst Method : C:IHPLC DatalrobinlMethod Robin 87H from C:IHPLC DatalrobinlEthanol_June 10-2013।Ethanol007.raw

Proc Method : C:IHPLC DatalrobinlMethod Robin 87H from c:Ihplc datalrobinlethanol_june 10-2013lethanol007.rst [Editing in Progress] Calib Method : C:IHPLC DatalrobinlMethod Robin 87H from c:Ihplc datalrobinlethanol_june 10-2013lethanol007.rst [Editing in Progress]

Report Format File: C:IHPLC DatalrobinlMethod Robin 87H.rpt

Sequence File : C:IHPLC DatalrobinIValera Sequence 87H-Etanol_June 10_2013.seq

\begin{tabular}{|c|c|c|c|c|c|c|c|}
\hline $\begin{array}{c}\text { Peak } \\
\quad \#\end{array}$ & $\begin{array}{l}\text { Time } \\
\text { [min] }\end{array}$ & $\begin{array}{l}\text { Area } \\
{[\mu \mathrm{V} \cdot \mathrm{s}]}\end{array}$ & $\begin{array}{l}\text { Height } \\
{[\mu \mathrm{V}]}\end{array}$ & $\begin{array}{c}\text { Area } \\
{[\%]}\end{array}$ & $\begin{array}{c}\text { Norm. Area } \\
{[\%]}\end{array}$ & $B L$ & $\begin{array}{c}\text { Area/Height } \\
\text { [s] }\end{array}$ \\
\hline - & 0.001 & 0.00 & 0.00 & 0.00 & 0.00 & & \\
\hline 1 & 0.100 & 354.00 & 122.61 & 0.01 & 0.01 & BB & 2.8872 \\
\hline 2 & 0.158 & 290.00 & 123.00 & 0.01 & 0.01 & BB & 2.3577 \\
\hline 3 & 0.244 & 365.20 & 57.49 & 0.01 & 0.01 & BB & 6.3528 \\
\hline 4 & 0.362 & 170.80 & 72.21 & 0.01 & 0.01 & BB & 2.3652 \\
\hline 5 & 0.418 & 351.69 & 148.13 & 0.01 & 0.01 & BV & 2.3742 \\
\hline 6 & 0.493 & 153.31 & 116.28 & 0.00 & 0.00 & VB & 1.3185 \\
\hline 7 & 0.544 & 72.80 & 66.95 & 0.00 & 0.00 & BB & 1.0873 \\
\hline 8 & 0.625 & 128.80 & 111.25 & 0.00 & 0.00 & BB & 1.1578 \\
\hline 9 & 0.678 & 296.40 & 123.12 & 0.01 & 0.01 & BB & 2.4073 \\
\hline 10 & 0.758 & 208.40 & 113.05 & 0.01 & 0.01 & BB & 1.8435 \\
\hline 11 & 0.804 & 77.20 & 69.43 & 0.00 & 0.00 & BB & 1.1120 \\
\hline 12 & 0.892 & 806.00 & 159.83 & 0.02 & 0.02 & BB & 5.0429 \\
\hline 13 & 1.178 & 140.29 & 84.58 & 0.00 & 0.00 & BV & 1.6586 \\
\hline 14 & 1.218 & 275.70 & 132.46 & 0.01 & 0.01 & VB & 2.0814 \\
\hline 15 & 1.403 & 282.40 & 75.14 & 0.01 & 0.01 & BB & 3.7582 \\
\hline 16 & 1.489 & 148.40 & 95.91 & 0.00 & 0.00 & BB & 1.5473 \\
\hline 17 & 1.573 & 232.40 & 89.66 & 0.01 & 0.01 & BB & 2.5919 \\
\hline 18 & 1.630 & 213.24 & 184.63 & 0.01 & 0.01 & BV & 1.1549 \\
\hline 19 & 1.656 & 317.67 & 166.35 & 0.01 & 0.01 & VV & 1.9097 \\
\hline 20 & 1.701 & 420.47 & 162.51 & 0.01 & 0.01 & VV & 2.5873 \\
\hline 21 & 1.784 & 560.35 & 117.20 & 0.02 & 0.02 & VV & 4.7813 \\
\hline 22 & 1.857 & 142.96 & 107.99 & 0.00 & 0.00 & VV & 1.3238 \\
\hline 23 & 1.888 & 128.31 & 103.88 & 0.00 & 0.00 & VB & 1.2352 \\
\hline 24 & 2.033 & 114.40 & 60.54 & 0.00 & 0.00 & BB & 1.8896 \\
\hline 25 & 2.176 & 65.60 & 61.79 & 0.00 & 0.00 & BB & 1.0617 \\
\hline 26 & 2.287 & 329.82 & 117.16 & 0.01 & 0.01 & BV & 2.8151 \\
\hline 27 & 2.358 & 336.58 & 94.51 & 0.01 & 0.01 & VB & 3.5613 \\
\hline 28 & 2.451 & 166.01 & 101.64 & 0.01 & 0.01 & BV & 1.6333 \\
\hline 29 & 2.523 & 157.59 & 79.99 & 0.00 & 0.00 & VB & 1.9702 \\
\hline 30 & 2.624 & 452.00 & 99.00 & 0.01 & 0.01 & BB & 4.5657 \\
\hline 31 & 2.833 & 346.03 & 76.90 & 0.01 & 0.01 & $\mathrm{BV}$ & 4.5000 \\
\hline 32 & 2.922 & 96.72 & 78.37 & 0.00 & 0.00 & VV & 1.2340 \\
\hline 33 & 2.953 & 84.05 & 76.53 & 0.00 & 0.00 & VB & 1.0983 \\
\hline 34 & 2.986 & 173.20 & 103.24 & 0.01 & 0.01 & BB & 1.6777 \\
\hline 35 & 3.056 & 89.20 & 68.23 & 0.00 & 0.00 & BB & 1.3074 \\
\hline 36 & 3.176 & 472.60 & 107.22 & 0.01 & 0.01 & BB & 4.4078 \\
\hline 37 & 3.322 & 111.80 & 81.39 & 0.00 & 0.00 & BB & 1.3737 \\
\hline 38 & 3.413 & 57.12 & 25.88 & 0.00 & 0.00 & BV & 2.2072 \\
\hline 39 & 3.474 & 103.68 & 51.40 & 0.00 & 0.00 & VB & 2.0173 \\
\hline 40 & 3.642 & 136.40 & 40.68 & 0.00 & 0.00 & BB & 3.3533 \\
\hline 41 & 3.783 & 76.60 & 71.99 & 0.00 & 0.00 & BB & 1.0640 \\
\hline 42 & 3.842 & 71.40 & 48.21 & 0.00 & 0.00 & BB & 1.4810 \\
\hline 43 & 3.981 & 61.20 & 62.00 & 0.00 & 0.00 & BB & 0.9871 \\
\hline 44 & 4.009 & 96.60 & 93.92 & 0.00 & 0.00 & BB & 1.0285 \\
\hline 45 & 4.108 & 114.40 & 65.75 & 0.00 & 0.00 & $\mathrm{BB}$ & 2.7500 \\
\hline
\end{tabular}




\begin{tabular}{|c|c|c|c|c|c|c|c|}
\hline $\begin{array}{c}\text { Peak } \\
\#\end{array}$ & $\begin{array}{l}\text { Time } \\
\text { [min] }\end{array}$ & $\begin{array}{l}\text { Area } \\
{[\mu \mathrm{V} \cdot \mathrm{s}]}\end{array}$ & $\begin{array}{l}\text { Height } \\
{[\mu \mathrm{V}]}\end{array}$ & $\begin{array}{c}\text { Area } \\
{[\%]}\end{array}$ & $\begin{array}{c}\text { Norm. Area } \\
{[\%]}\end{array}$ & $B L$ & $\begin{array}{c}\text { Area/Height } \\
{[\mathrm{s}]}\end{array}$ \\
\hline 46 & 4.208 & 57.76 & 56.14 & 0.00 & 0.00 & BV & 1.0288 \\
\hline 47 & 4.261 & 386.04 & 86.76 & 0.01 & 0.01 & VB & 4.4496 \\
\hline 48 & 4.475 & 268.80 & 81.16 & 0.01 & 0.01 & BB & 3.3120 \\
\hline 49 & 4.703 & 268.60 & 107.73 & 0.01 & 0.01 & BB & 2.4933 \\
\hline 50 & 4.970 & 210.20 & 50.49 & 0.01 & 0.01 & BB & 4.1634 \\
\hline 51 & 5.415 & 166.40 & 61.23 & 0.01 & 0.01 & $\mathrm{BB}$ & 2.7176 \\
\hline 52 & 6.200 & 38076.60 & 2067.58 & 1.15 & 1.15 & BB & 18.4161 \\
\hline 53 & 7.505 & 174466.02 & 3923.52 & 5.25 & 5.25 & BV & 44.4667 \\
\hline 54 & 7.635 & 24377.53 & 2438.47 & 0.73 & 0.73 & VV & 9.9971 \\
\hline 55 & 7.718 & 6990.32 & 1434.87 & 0.21 & 0.21 & VV & 4.8718 \\
\hline 56 & 7.764 & 2550.41 & 932.51 & 0.08 & 0.08 & VV & 2.7350 \\
\hline 57 & 7.822 & 1124.54 & 255.61 & 0.03 & 0.03 & VB & 4.3994 \\
\hline 58 & 7.965 & 285.60 & 102.23 & 0.01 & 0.01 & BB & 2.7936 \\
\hline 59 & 8.025 & 119.30 & 76.57 & 0.00 & 0.00 & BV & 1.5581 \\
\hline 60 & 8.069 & 107.85 & 72.03 & 0.00 & 0.00 & VV & 1.4973 \\
\hline 61 & 8.158 & 278.33 & 78.54 & 0.01 & 0.01 & VV & 3.5438 \\
\hline 62 & 8.207 & 41.33 & 39.73 & 0.00 & 0.00 & VB & 1.0401 \\
\hline 63 & 8.294 & 325.33 & 163.98 & 0.01 & 0.01 & BV & 1.9840 \\
\hline 64 & 8.375 & 283.47 & 87.80 & 0.01 & 0.01 & VB & 3.2287 \\
\hline 65 & 8.410 & 46.60 & 48.06 & 0.00 & 0.00 & BB & 0.9696 \\
\hline 66 & 8.472 & 56.40 & 36.37 & 0.00 & 0.00 & BB & 1.5506 \\
\hline 67 & 8.559 & 260.40 & 117.44 & 0.01 & 0.01 & BB & 2.2173 \\
\hline 68 & 8.694 & 199.20 & 86.12 & 0.01 & 0.01 & $\mathrm{BB}$ & 2.3131 \\
\hline 69 & 8.772 & 202.40 & 100.04 & 0.01 & 0.01 & $\mathrm{BB}$ & 2.0231 \\
\hline 70 & 8.914 & 262.00 & 82.57 & 0.01 & 0.01 & $\mathrm{BB}$ & 3.1731 \\
\hline 71 & 8.959 & 329.67 & 181.27 & 0.01 & 0.01 & BV & 1.8186 \\
\hline 72 & 9.052 & 731.93 & 105.07 & 0.02 & 0.02 & VB & 6.9658 \\
\hline 73 & 9.262 & 605.00 & 128.68 & 0.02 & 0.02 & BB & 4.7017 \\
\hline 74 & 9.340 & 80.02 & 75.21 & 0.00 & 0.00 & $\mathrm{BV}$ & 1.0639 \\
\hline 75 & 9.367 & 74.38 & 72.19 & 0.00 & 0.00 & VB & 1.0302 \\
\hline 76 & 9.401 & 478.40 & 113.65 & 0.01 & 0.01 & BB & 4.2093 \\
\hline 77 & 9.620 & 178.00 & 122.27 & 0.01 & 0.01 & $\mathrm{BB}$ & 1.4558 \\
\hline 78 & 9.759 & 239.20 & 69.10 & 0.01 & 0.01 & BB & 3.4619 \\
\hline 79 & 9.889 & 98.40 & 82.66 & 0.00 & 0.00 & $\mathrm{BB}$ & 1.1905 \\
\hline 80 & 10.160 & 76.60 & 79.61 & 0.00 & 0.00 & $\mathrm{BB}$ & 0.9622 \\
\hline 81 & 10.451 & 43.60 & 60.63 & 0.00 & 0.00 & $\mathrm{BB}$ & 0.7192 \\
\hline 82 & 10.533 & 132.80 & 85.45 & 0.00 & 0.00 & $\mathrm{BB}$ & 1.5541 \\
\hline 83 & 10.563 & 376.21 & 109.57 & 0.01 & 0.01 & BV & 3.4336 \\
\hline 84 & 10.702 & 226.98 & 87.54 & 0.01 & 0.01 & VV & 2.5929 \\
\hline 85 & 10.768 & 253.21 & 70.40 & 0.01 & 0.01 & VB & 3.5966 \\
\hline 86 & 10.834 & 216.30 & 87.73 & 0.01 & 0.01 & BV & 2.4655 \\
\hline 87 & 10.907 & 129.70 & 50.75 & 0.00 & 0.00 & VB & 2.5558 \\
\hline 88 & 11.110 & 195.60 & 66.30 & 0.01 & 0.01 & BB & 2.9500 \\
\hline 89 & 11.186 & 222.52 & 107.14 & 0.01 & 0.01 & BV & 2.0768 \\
\hline 90 & 11.239 & 295.48 & 112.03 & 0.01 & 0.01 & VB & 2.6374 \\
\hline 91 & 11.511 & 1856.00 & 251.41 & 0.06 & 0.06 & BB & 7.3825 \\
\hline 92 & 11.649 & 357.60 & 95.46 & 0.01 & 0.01 & BB & 3.7461 \\
\hline 93 & 11.917 & 137.40 & 75.77 & 0.00 & 0.00 & $\mathrm{BB}$ & 1.8134 \\
\hline 94 & 12.189 & 148.21 & 69.64 & 0.00 & 0.00 & BV & 2.1282 \\
\hline 95 & 12.243 & 104.99 & 76.33 & 0.00 & 0.00 & VB & 1.3755 \\
\hline 96 & 12.481 & 583.80 & 101.03 & 0.02 & 0.02 & BB & 5.7783 \\
\hline 97 & 12.615 & 232.60 & 59.35 & 0.01 & 0.01 & BB & 3.9189 \\
\hline 98 & 12.759 & 540.60 & 95.33 & 0.02 & 0.02 & $\mathrm{BB}$ & 5.6708 \\
\hline 99 & 12.907 & 584.39 & 119.65 & 0.02 & 0.02 & BV & 4.8842 \\
\hline 100 & 13.007 & 144.01 & 75.57 & 0.00 & 0.00 & VB & 1.9057 \\
\hline 101 & 13.128 & 248.40 & 52.60 & 0.01 & 0.01 & BB & 4.7227 \\
\hline 102 & 13.215 & 95.60 & 76.58 & 0.00 & 0.00 & $\mathrm{BB}$ & 1.2483 \\
\hline 103 & 13.523 & 136.76 & 71.72 & 0.00 & 0.00 & BV & 1.9069 \\
\hline 104 & 13.588 & 60.44 & 53.74 & 0.00 & 0.00 & VB & 1.1247 \\
\hline 105 & 13.641 & 49.40 & 49.02 & 0.00 & 0.00 & $\mathrm{BB}$ & 1.0077 \\
\hline 106 & 13.956 & 51.20 & 49.22 & 0.00 & 0.00 & $\mathrm{BB}$ & 1.0402 \\
\hline 107 & 14.071 & 242.40 & 93.32 & 0.01 & 0.01 & BB & 2.5976 \\
\hline 108 & 14.157 & 146.09 & 56.12 & 0.00 & 0.00 & BV & 2.6032 \\
\hline 109 & 14.230 & 464.71 & 99.32 & 0.01 & 0.01 & VB & 4.6788 \\
\hline 110 & 14.415 & 356.00 & 73.43 & 0.01 & 0.01 & $\mathrm{BB}$ & 4.8484 \\
\hline 111 & 14.555 & 338.67 & 109.91 & 0.01 & 0.01 & BV & 3.0813 \\
\hline 112 & 14.604 & 245.73 & 118.40 & 0.01 & 0.01 & VB & 2.0755 \\
\hline 113 & 15.218 & 221.60 & 60.99 & 0.01 & 0.01 & BB & 3.6335 \\
\hline 114 & 15.251 & 63.60 & 54.36 & 0.00 & 0.00 & BB & 2.1801 \\
\hline
\end{tabular}




\begin{tabular}{|c|c|c|c|c|c|c|c|}
\hline $\begin{array}{c}\text { Peak } \\
\#\end{array}$ & $\begin{array}{l}\text { Time } \\
\text { [min] }\end{array}$ & $\begin{array}{l}\text { Area } \\
{[\mu \mathrm{V} \cdot \mathrm{s}]}\end{array}$ & $\begin{array}{l}\text { Height } \\
{[\mu \mathrm{V}]}\end{array}$ & $\begin{array}{c}\text { Area } \\
{[\%]}\end{array}$ & $\begin{array}{c}\text { Norm. Area } \\
{[\%]}\end{array}$ & $\mathrm{BL}$ & $\begin{array}{c}\text { Area/Height } \\
{[\mathrm{s}]}\end{array}$ \\
\hline 115 & 15.368 & 181.20 & 68.39 & 0.01 & 0.01 & BB & 2.6494 \\
\hline 116 & 15.517 & 264.00 & 89.09 & 0.01 & 0.01 & BB & 2.9632 \\
\hline 117 & 15.826 & 590.60 & 124.98 & 0.02 & 0.02 & BB & 4.7257 \\
\hline 118 & 15.989 & 126.20 & 48.25 & 0.00 & 0.00 & BB & 2.6154 \\
\hline 119 & 16.223 & 259.60 & 39.14 & 0.01 & 0.01 & BB & 6.6318 \\
\hline 120 & 16.322 & 107.60 & 42.96 & 0.00 & 0.00 & BB & 2.5047 \\
\hline 121 & 16.636 & 86.40 & 47.74 & 0.00 & 0.00 & BB & 1.8099 \\
\hline 122 & 16.837 & 263.60 & 39.39 & 0.01 & 0.01 & BB & 6.6918 \\
\hline 123 & 16.968 & 289.20 & 61.12 & 0.01 & 0.01 & BB & 4.7319 \\
\hline 124 & 17.159 & 203.20 & 41.20 & 0.01 & 0.01 & BB & 4.9324 \\
\hline 125 & 17.286 & 146.40 & 55.12 & 0.00 & 0.00 & BB & 2.6559 \\
\hline 126 & 17.430 & 91.80 & 51.77 & 0.00 & 0.00 & BB & 1.7731 \\
\hline 127 & 17.761 & 147.00 & 51.34 & 0.00 & 0.00 & BB & 2.8630 \\
\hline 128 & 17.822 & 71.60 & 44.59 & 0.00 & 0.00 & BB & 1.6059 \\
\hline 129 & 18.085 & 171.40 & 83.19 & 0.01 & 0.01 & BB & 2.0602 \\
\hline 130 & 18.658 & 104.83 & 62.72 & 0.00 & 0.00 & BV & 1.6716 \\
\hline 131 & 18.744 & 219.37 & 61.29 & 0.01 & 0.01 & VB & 3.5789 \\
\hline 132 & 18.843 & 202.00 & 40.47 & 0.01 & 0.01 & BB & 4.9914 \\
\hline 133 & 18.883 & 159.22 & 98.73 & 0.00 & 0.00 & BV & 1.6127 \\
\hline 134 & 18.962 & 321.34 & 111.00 & 0.01 & 0.01 & VV & 2.8950 \\
\hline 135 & 18.987 & 118.44 & 111.57 & 0.00 & 0.00 & VV & 1.0615 \\
\hline 136 & 19.105 & 1024.22 & 149.35 & 0.03 & 0.03 & VV & 6.8581 \\
\hline 137 & 19.155 & 368.17 & 143.09 & 0.01 & 0.01 & VV & 2.5730 \\
\hline 138 & 19.246 & 726.98 & 130.85 & 0.02 & 0.02 & VV & 5.5560 \\
\hline 139 & 19.285 & 359.23 & 162.54 & 0.01 & 0.01 & VV & 2.2101 \\
\hline 140 & 19.369 & 353.18 & 103.37 & 0.01 & 0.01 & VV & 3.4165 \\
\hline 141 & 19.423 & 197.43 & 80.21 & 0.01 & 0.01 & VB & 2.4614 \\
\hline 142 & 19.555 & 275.17 & 71.70 & 0.01 & 0.01 & BV & 3.8380 \\
\hline 143 & 19.690 & 586.00 & 164.62 & 0.02 & 0.02 & VB & 3.5597 \\
\hline 144 & 19.833 & 159.94 & 97.13 & 0.00 & 0.00 & BV & 1.6467 \\
\hline 145 & 19.867 & 226.06 & 81.62 & 0.01 & 0.01 & VB & 2.7696 \\
\hline 146 & 20.007 & 84.80 & 62.97 & 0.00 & 0.00 & BB & 1.3466 \\
\hline 147 & 20.175 & 200.02 & 66.88 & 0.01 & 0.01 & BV & 2.9906 \\
\hline 148 & 20.364 & 195.12 & 71.22 & 0.01 & 0.01 & VB & 2.7395 \\
\hline 149 & 20.504 & 182.15 & 72.48 & 0.01 & 0.01 & BV & 2.5131 \\
\hline 150 & 20.556 & 85.25 & 50.58 & 0.00 & 0.00 & VB & 1.6855 \\
\hline 151 & 20.637 & 130.40 & 82.69 & 0.00 & 0.00 & $\mathrm{BB}$ & 1.5770 \\
\hline 152 & 20.771 & 81.18 & 70.29 & 0.00 & 0.00 & BV & 1.1550 \\
\hline 153 & 20.831 & 730.99 & 145.77 & 0.02 & 0.02 & VV & 5.0146 \\
\hline 154 & 21.582 & 3027716.93 & 76668.46 & 91.20 & 91.20 & VV & 39.4910 \\
\hline 155 & 22.862 & 262.30 & 102.82 & 0.01 & 0.01 & VB & 2.5509 \\
\hline 156 & 23.011 & 101.20 & 46.84 & 0.00 & 0.00 & $\mathrm{BB}$ & 2.1606 \\
\hline 157 & 23.144 & 212.28 & 87.02 & 0.01 & 0.01 & BV & 2.4394 \\
\hline 158 & 23.228 & 120.03 & 75.39 & 0.00 & 0.00 & VV & 1.5920 \\
\hline 159 & 23.281 & 299.69 & 78.94 & 0.01 & 0.01 & VB & 3.7966 \\
\hline 160 & 23.445 & 280.60 & 68.22 & 0.01 & 0.01 & $\mathrm{BB}$ & 4.1133 \\
\hline 161 & 23.567 & 256.00 & 72.13 & 0.01 & 0.01 & BB & 3.5489 \\
\hline 162 & 23.636 & 1564.20 & 442.18 & 0.05 & 0.05 & BV & 3.5375 \\
\hline 163 & 23.701 & 2834.59 & 447.88 & 0.09 & 0.09 & VV & 6.3290 \\
\hline 164 & 23.844 & 1821.14 & 288.87 & 0.05 & 0.05 & VV & 6.3044 \\
\hline 165 & 24.026 & 113.67 & 37.91 & 0.00 & 0.00 & VB & 2.9989 \\
\hline 166 & 24.176 & 158.40 & 56.39 & 0.00 & 0.00 & BB & 2.8088 \\
\hline 167 & 24.344 & 169.20 & 70.92 & 0.01 & 0.01 & BB & 2.3857 \\
\hline 168 & 24.620 & 69.00 & 41.07 & 0.00 & 0.00 & BB & 1.6800 \\
\hline \multirow[t]{2}{*}{169} & 24.863 & 278.80 & 50.07 & 0.01 & 0.01 & $\mathrm{BB}$ & 5.5678 \\
\hline & & & & & & & \\
\hline
\end{tabular}

Warning -- Signal level out-of-range in peak

Missing Component Report

Component Expected Retention (Calibration File)

standards

0.001 

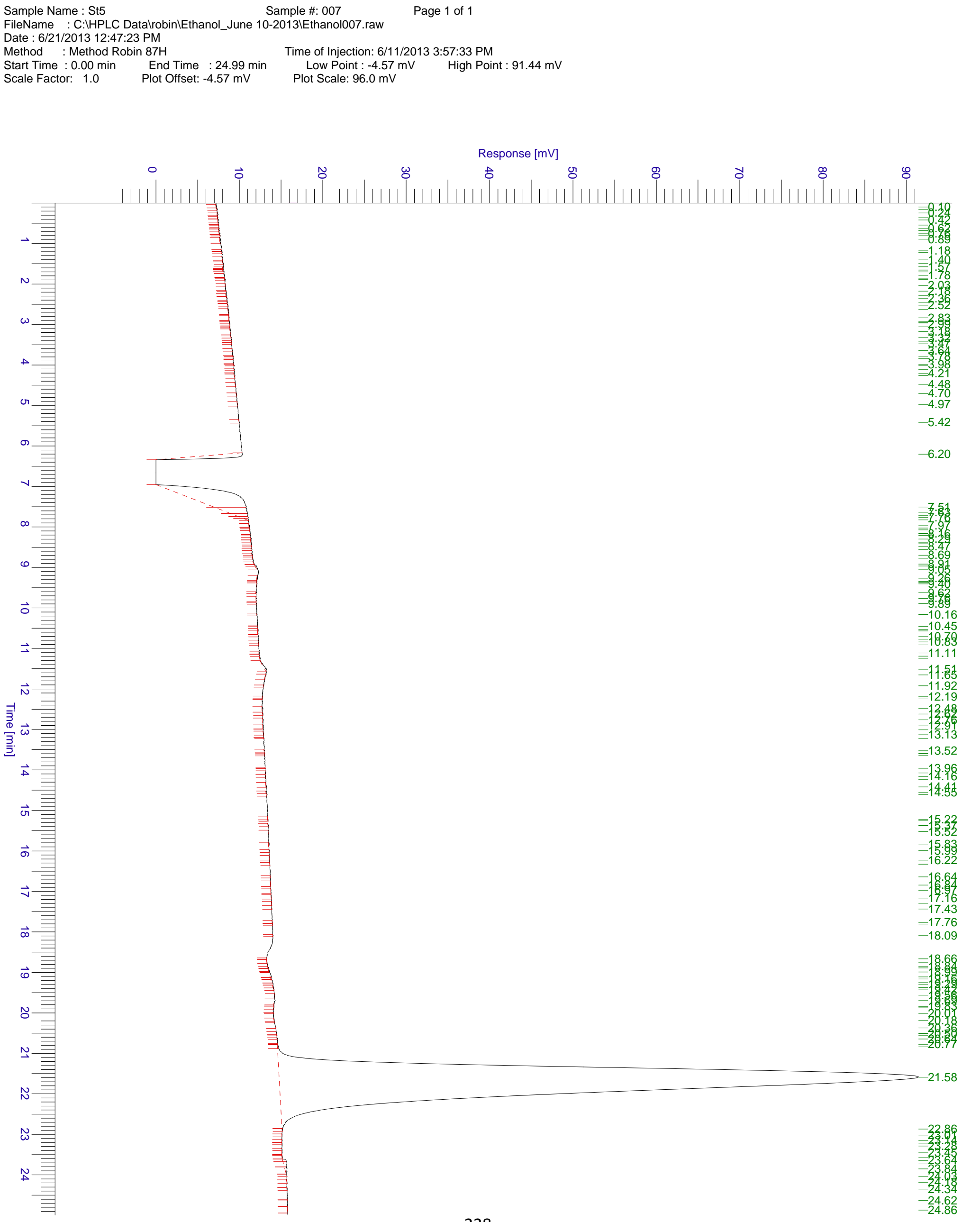


\section{REFERENCES}

Aiba, S., Shoda, M., \& Nagatani, M. (1968). Kinetics of product inhibition in alcohol fermentation. Biotechnology and Bioengineering, 10(6), 845-864.

Alvira, P., Tomás-Pejó, E., Ballesteros, M., \& Negro, M.J. (2010). Pretreatment technologies for an efficient bioethanol production process based on enzymatic hydrolysis. A review. Bioresource Technology, 101(13), 4851-4861.

Ballestaros, I., Ballestaros, M., Cabaoas, A., Carrasco, J., Martin, C., Negro, M. J., Saez, F., \& Saez, R. (1991). Selection of thermotolerant yeasts for simultaneous saccharification and fermentation (SSF) of cellulose to ethanol. Applied Biochemistry and Biotechnology, 28/29, 307-315.

Banerjee, S., Mudliar, S., Sen, R., \& Giri, B. (2010). Commercializing lignocellulosic bioethanol: technology bottlenecks and possible remedies. Biofuels, Bioproducts, Biorefinery, 4, 77-93.

Barron, N., Marchant, R., McHale, L., \& McHale, A. P. (1995). Studies on the use of thermotolerant strain of Kluyveromyces marxianus insimultaneous sacharification and ethanol formation from cellulose. Applied Microbiology and Biotechnology, 43, 518-520.

Bazua, Z. (1977). Ethanol effect on the kinetics of a continuos fermentation with Saccharomyces cerevisiae. Biotechnology and Bioengineering, 7, 105-118.

Bekmuradov, V., Luk, G., \& Luong, R. (2014a). Improved cellulose and organic-solvent-based lignocellulosic fractionation pre-treatment of organic waste for bioethanol production. American Journal of Engineering Research, 3(6), 177-185. Available from: http://www.ajer.org/papers/v3\%286\%29/U036177185.pdf 
Bekmuradov, V., Luk, G., \& Luong, R. (2014b). Comparative ethanol productivities of two different recombinant fermenting strains on source separated organic waste. International Journal of Engineering Research and Applications, 4(10), 77-82. Retrieved from: http://www.ijera.com/pages/v4no10(v5).html

Bretscher, O. (1995). Linear algebra with applications, $3^{\text {rd }}$ edition. Upper Saddle River. NJ: Prentice Hall.

Brown, L., \& Torget, R. (1996). Enzymatic saccharification of lignocellulosic biomass. Chemical and Testing Task: Laboratory Analytical Procedure LAP-009. Technical report. Golden, Colorado, USA: National Renewable Energy Laboratory. Retrieved from: http://www.p2pays.org/ref/40/39116.pdf

Cadoche, L., \& Lopez, J. D. (1989). Assessment of size reduction as a preliminary step in production of ethanol from lignocellulosic wastes. Biological Wastes, 30, 153-157.

Chandrakant, P., \& Bisaria, V.S. (1998). Simultaneous bioconversion of cellulose and hemicelluloses to ethanol. Critical Review Biotechnology, 18, 295-331.

Chang, V. S., \& Holtzapple, M. T. (2000). Fundamental factors affecting biomass enzymatic reactivity. Applied Biochemistry and Biotechnology, 84-86, 5-37.

Chu, B., \& Lee, H. (2007). Genetic improvement of Saccharomyces cerevisiae for xylose fermentation. Biotechnology Advances, 25(5), 425-441.

Collins, K. (2007). The new world of biofuels: implications for agriculture and energy. Energy Information Administration/USDA. Energy Outlook, Modeling, and Data Conference, Washington, DC: Prentice Hall. Retrieved from: http://www.eia.doe.gov/oiaf/aeo/conf/collins/collins_pres.html. 
Dale, B. E., Henk, L. L., \& Shiang, M. (1984). Fermentation of lignocellulosic material treated by ammonia freeze explosion. Developments in Industrial Microbiology, 26, 223-233.

Dale, M. C., \& Moelhman, M. (2001). Enzymatic simultaneous saccharification and fermentation (SSF) of biomass to ethanol in a pilot 130 liter multistage continuous reactor separator. $9^{\text {th }}$ Biennial Bioenergy Conference “Moving technology into marketplace”. Buffalo, NY, USA.

Dean, A. R. C. (1964). Growth, function and regulation in bacterial cells.London, UK: Oxford University Press:

Deanda, K., Zhang, M., Eddy, S., \& Piccatagio, S. (1996). Development an arabinose fermenting Zymomonas mobilis strain by metabolic pathway engineering. Applied Environmental Microbiology, 62, 4465-4470.

Delgenes, J. P., Molleta, R., \& Navarro, J. M. (1996). Effects of lignocellulosic degradation products on ethanol fermentation of glucose and xylose by Saccharomyces cerevisiae, Zymomonas mobilis, Pichia stipitis and Candida shehatae. Enzyme and Microbial Technology, 19, 220-225.

Dellweg, H., Rizzi, M., Methner, H., \& Debus, D. (1984). Xylose fermentation by yeasts. Biotechnology Letters, 6, 395-400.

Demain, A.L., Newcomb, M., \&Wu, J.H. (2005). Cellulase, clostridia, and ethanol. Microbiology and Molecular Biology Reviews, 69(1), 124-154.

Dowe, N. (2009). Assessing cellulose performance on pretreated lignocellulosic biomass using saccharification and fermentation based protocols. Methods in Molecular Biology, 581, 233-245. 
Dowe, N., \& McMillan, J.D. (2008). SSF experimental protocols - lignocellulosic biomass hydrolysis and fermentation. Laboratory analytical procedure (LAP NREL/TP-51042630). Technical report. Golden, Colorado, USA: National Renewable Energy Laboratory.Retrieved from: http://www.nrel.gov/docs/gen/fy08/42630.pdf

Duff, S. J. B., \& Murray, W. D. (1996). Bioconversion of forest products industry waste cellulosic to fuel ethanol. Review. Bioresource Technology, 55, 1-33.

du Preez, J. C., van Driessel, B., \& Prior, B.A. (1989). Ethahol tolerance of Pichia stipitis and Candida shehatae strains in batch-fed cultures at controlled low dissolved oxygen levels. Applied Microbiology and Biotechnology, 30, 53-58.

du Preez, J. C., Bosch, M., \& Prior, B. A. (1986). The fermentation of hexose and pentose sugars by Candida shehatae and Pichia stipitis. Applied Microbiology and Biotechnology, 23, 228-233.

du Preez, J. C., Kock, J. L. F., Monteiro, A. M. T., \& Prior, B. A. (1985). The vitamin requirements of Candida shehatae for xylose fermentation. FEMS Microbiology Letters, 28, 271-275.

Ehsanipour, M. (2010). Acid pretreatment and fractionation of source separated organic waste for lignocellulosic sacharification (Master’s thesis). Ryerson University, Civil Engineering Dept., Toronto, Canada.

European Biomass Industry Association (EUBIA) (2007). Biomass characteristics. What is biomass. Brussels, Belgium: EUBIA. Retrieved from: http://www.eubia.org/115.0.html Fan, L. T., Gharpuray, M. M., \& Lee, Y. H. (1987).Cellulose hydrolysis. Biotechology monographs, vol. 3 (pp. 1 -198). Berlin, New -York, London, Paris and Tokio: SpringerVerlag, Heidelberg. 
Fan, Z., South, S., Lyford, K., Munsie, J., van A Walsum, P., \& Lynd, L. (2003). Conversion of paper sludge to ethanol in a semicontinuous solids-fed reactors. Bioprocess Biosystems Engineering, 26(2), 93-101.

Faye, M. (2010). Chemical pretreatment and enzymatic hydrolysis of mixed source-separated organic (SSO) and wood waste (Master's thesis). Ryerson University, Civil Engineering Dept., Toronto, Canada.

Ferreira, J. A., Lennartsson, P.R., \& Taherzade, M. J. (2014). Production of ethanol and biomass from thin stillage using food-grade Zygomycetes and Ascomycetes filamentous fungi. Energies, 7, 3872-3885

Ge, X., Burner, D.M., Xu, J., Phillips, G.C., \& Sivakumar, G. (2011). Bioethanol production from dedicated energy crops and residues in Arkansas, USA. Biotechnology, 6, 66-73.

Ghose, T. K. (1987). Measurement of cellulose activities. Pure Applied Chemistry, 59(2), 257268.

Goldstein, I., \& Easter, J. (1992). An improved process for converting cellulose to ethanol. Tappi Journal, 28, 135-142.

Hahn-Hagerdal, B., Hallborn, J., Jeppsson, H., Olsson, L., Skoog, K., \& Walfridsson, M. (1993). Pentose fermentation to alcohol. In J.N., Saddler (Ed), Bioconversion in forest and agricultural plant residues (pp. 231-290). C.A.B. Wallinford, UK: Oxford University Press.

Hahn-Hagerdal, B., Jeppsson, H., Olsson, L., \& Mohagheghi, A. (1994a). An interlaboratory comparison of the performance of ethanol producing micro-organisms in a xylose rich acid hydrolysate. Applied Microbiology and Biotechnology, 41, 62-67. 
Hahn-Hagerdal, B., Jeppsson, H., Skoog, K., \& Prior, B. A. (1994b). Biochemistry and physiology of xylose fermentation by yeasts. Enzyme and Microbial Technology, 16, 933-943.

Hames, B., Ruiz, R., Scarlata, C., Sluiter, A., Sluiter, J., \& Templeton, D. (2008). Preparation of samples for compositional analysis. Laboratory Analytical Procedure (LAP).NREL/TP510-42620.Technical Report. Golden, Colorado, USA: National Renewable Energy Laboratory: Retrieved from http://www.nrel.gov/docs/gen/fy08/42620.pdf

Harvey, S., Chambers, A., \& Zhang, P. (2008). Overproduction of hydrogen from an aerobic bacterium. Proceedings of the Army Science Conference (26th), Orlando, Florida, USA. Hinman, N.D., Wright, J.D., Hoagland, W., \& Wyman, C.E. (1989).Xylose fermentation: an economic analysis. Applied Biochemical Biotechnology, 20(21), 391-401.

Ho, N. W. Y., Chen, Z., \& Brainard, A. (1998). Genetically engineered Saccharomyces yeast capable of effective co-fermentation of glucose and xylose. Applied Environment Microbiology, 64, 1852-1859.

Holtzaplle, M. T. (1993). Cellulose, hemicellulose and lignin. In R., Macrae, R., Robinson, K. J.M., \& Saddler (Eds). Encyclopedia in food science, food techology, and nutrition (pp.758-767, 2324-2334, 2731-2738). London, UK: Academic Press.

Hougton, J., Weatherwax, S., \& Ferrell, J. (2006). Breaking in biological barriers to cellulosic ethanol: A joint research agenda. Rockville, Maryland: US Department of Energy. Ingram, L. O. (1986). Microbial tolerance to alcohols: role of the cell membrane. Trends in Biotechnology, 4, 40-44.

Jeffries, T. W. (1983). Utilization of xylose by bacteria, yeasts, and fungi. Advances in Biochemical Engineering/Biotechnology, 38, 1-32. 
Joachimsthal, E., Haggett, K. D., \& Rogers, P. L. (1999). Evolution of recombinant strains of Zymomonas mobilis for ethanol production from glucose/xylose media. Applied Biochemistry and Biotechnology, 77-79, 147-157.

Johansson, T. B., Kelly, H., Reddy, K. N., \& Williams, R. H. (1993). Renewable fuels and elecricity for a growing world economy: defining and achieving the potential. InJohansson, T.B., Kelly, H., Reddy, A.K.N., \& Williams, R.H., (Eds.). Renewable energy: Sources for fuels and electricity. Washington, DC: Island Press.

Kim, I. S., Barrow, K. D., \& Rogers, P. L. (2000). Kinetic and nuclear magnetic resonance studies of xylose metabolism by recombinant Zymomonas mobilis ZM4 (pZB5). Applied and Environmental Microbiology, 66(1), 186-193.

Kim, T. Y., \& Lee, Y. Y. (2005). Pretreatment of corn stover by soaking in aqueous ammonia. Applied Biochemistry and Biotechnology, 121, 1119-1131.

Kompala, D., McMillan, J., \& Zhang, M. (2001). Maximizing etahnol production by engineered pentose fermenting Zymomonas mobilis. Golden, Colorado, USA: Biotechnology Center for Fuels and Chemicals, National Renewable Energy Laboratory.Retrieved from: http://www.metabolicengineering.gov/me2001/2001Kompala.pdf.

Kumar, S., Singh, S. P., Mishra, I. M., \& Adhikari, D. K. (2009). Recent advances in ethanol production from lignocellulosic biomass. Chemical Engineering and Technology, 32(4), 517-526.

Kuyper, M., Toirkens, M.J., Diderich, J.A., Winkler, A.A., van Dijken, J.P., \& Pronk, J.T. (2005). Evolutionary engineering of mixed-sugar utilization by xylose fermenting Saccharomyces cerevisiae strain. FEMS Yeast Research, 5(10), 925-934. 
Kuyper, M., Winkler, A.A., van Dijken, J.P., \& Pronk, J.T. (2004). Minimal metabolic engineering of Saccharomyces cerevisiae for efficient anaerobic xylose fermentation: a proof of principle. FEMS Yeast Research, 4, 655-664.

Jansen, J., Spliid, H., Hansen, T. L., Svard, A., \& Christensen, T. H. (2004). Assessment of sampling and chemical analysis of source-separated organic household waste. Waste Management, 24, 541-549.

Ladisch, M.,\& Svartzkopf, J. (1991). Ethanol production and the cost of fermentable sugars from biomass. Bioresource Technology 36(1), 83-95.

Lawford, H.G., \& Rousseau, J.D. (2000). Comparative energetics of glucose and xylose metabolism in recombinant Zymomonas. Applied Biochemical Biotechnology, 84-86, 277-294.

Lin, Y., Zhang, W., Li, C., Sakakibara, K., Tanaka, S., \& Kong, H.(2012). Factors affecting ethanol fermentation using S. cerevisiae BY4742. Biomass and Bioenergy, 47, 395-401

Linden, T., \& Hahn-Hagerdal, B. (1989). Fermentation on lignocellulosic hydrolysates with yeasts and xylose isomerase. Enzyme and Microbial Technology, 11, 583-589.

Lindsay, S. E., Bothast, R. J., \& Ingram, L. O. (1995). Improved strains of recombinant Escherichia coli for ethanol production from sugar mixtures. Applied and Environmental Microbiology, 43, 70-75.

Liu, C., \& Wyman, C.E. (2005). Partial flow of compressed hot water through corn stover to enhance hemicelluloses sugar recovery and enzymatic digestibility of cellulose. Bioresource Technology, 96, 1978-1985. 
Liu, H., Zhu, J.Y., \& Chai, X.S. (2011). In situ, rapid, and temporally resolved measurements of cellulase adsorption onto lignocellulosic substrates by UV-Vis spectrophotometry, Langmuir, 27(1), 272-278.

Luong, R. (2012). The feasibility of converting pretreated source-separated organic (SSO) into bioethanol (Master’s thesis). Ryerson University, Civil Engineering Dept., Toronto, Canada.

Lynd, L. R., Cushman, J. H., Nichols, R. J., \& Wyman, E. E. (1991). Fuel ethanol from cellulosic biomass. Science, 251, 1318-1323.

Lynd, L. R., Elander, R. T., \& Wyman, C. E. (1996). Likely features and cost of mature biomass technology. Applied Biochemistry and Biotechnology, 57/58, 741-761.

Lynd, L.R., Lyford, K., South, C.R., \& Levenson, K. (2001). Evaluation of paper sludge for amenability to enzymatic hydrolysis and conversion to ethanol. Tappi Journal, 84, 50.

Lynd, L. R. (2008). Energy biotechnology. Current Opinion in Biotechnology, 19 (3), 199-201

Lynd, L.R., Weimar, P.J., van Zyl, W.H., \& Pretorius, I.S. (2002). Microbial cellulose utilization: fundamentals and biotechnology. Microbiology and Molecular Biology Reviews, 66(3), 506-677.

Lynd, L. R., Wyman, C. E., \& Gerngross, T. U. (1999). Biocommodity engineering. Biotechnology Progress, 15(5), 777-793.

Martin, C., \& Jonsson, L. (2003). Comparison of the resistance of industrial and laboratory strains of Saccharomyces and Zygo Saccharomyces to lignocellulosic derived fermentation inhibitors. Enzyme and Microbial Technology, 32(4), 386-395. 
McMillan, J. D. (1994). Pretreatment of lignocellulosic biomass. In: M.E. Himmel, J.O. Baker and R.A. Overend (Eds). Enzymatic conversion of biomass for fuels production (pp. 292324). Washington, DC: American Chemical Society.

McMillan, J. D., Newman, M. M., Templeton, D. W., \& Mohagheghi, A. (1999). Simultaneous saccharification and co-fermentation of dilute acid pretreated yellow poplar hardwood to ethanol using xylose fermenting Zymomonas mobilis. Applied Biochemistry and Biotechnology, 77-79, 649-665.

Mirzajani, M. (2009). The amenability of pre-treated source-separated organic (SSO) waste for ethanol production (Master’s thesis) Ryerson University, Civil Engineering dept., Toronto, Canada.

Thermo Fisher Scientific Inc. 2011.Modified Lowry protein assay kit. Retrieved from: http://www.thermoscientific.com/pierce.

Martín, C., Marcet, M., Almazán, O., \& Jönsson, L. J. (2007). Adaptation of a recombinant xylose-utilizing Saccharomyces cerevisiae strain to a sugarcane bagasse hydrolysate with high content of fermentation inhibitors. Bioresource Technology, 98: 1767-1773.

Mohagheghi, A., Dowe, N., Schell, D., Chou, Y., Eddy, C., \& Zhang, M. (2004). Performance of newly developed integrant of Zymomonas mobilis for ethanol production on corn stover hydrolysate. Biotechnology Letters, 26, 321-325.

Mohagheghi, A., Linger, J. G., Yang, S., Smith, H., Dowe, N., Zhang, M., \& Pienkos, F.T. (2015). Improving a recombinant Zymomonas mobilis strain 8b through continuous adaptation on dilute acid pretreated corn stover hydrolysate. Biotechnology for Biofuels,8, 55, $1-9$. 
Moniruzzaman, M., Dale, B. E., Hespell, R. B., \& Bothast, R. J. (1997). Enzymatic hydrolysis of high moisture corn fiber pretreated by APEX and recovery and recycling of enzyme complex. Applied Biochemistry and Biotechnology, 67(1-2), 113-126.

Mosier, N. S., Hall, P., Ladisch, C. M., \& Ladisch, M. R. (1999). Reaction kinetics, molecular action, and mechanisms of cellylotic proteins. Advances in Biochemical Engineering, 65, $24-40$.

Mosier, N., Wyman, C.E., Dale, B.E., Elander, R.T., Lee, Y.Y., Holtzapple, M., \& Ladisch, M. (2005). Feature of the promising technologies for pretreatment lignocellulosic biomass. Bioresource Technology, 96, 673-686.

Nguyen, Q. A., Dickov, J. H., Duff, B. W., Farmer, J. D., Glassner, D. A., Ibsen, K. N., Ruth, M. F., Schell, D. J., Thompson, A. B., \& Tucker, M. P. (1996). NREL/DOE ethanol pilot plan: current status and capabilities. Bioresource Technology, 58, 189-196.

Nilsson, U., Barron, N., McHale, L., \& McHale, A.P. (1995). The effect of phosphoric acid pretreatment on conversion of cellulose to ethanol at $45^{\circ} \mathrm{C}$ using thermotolerant yeast Kluyveromyces marxianus IMB3. Biotechnology Letters, 17, 985-988.

Optimum waste \& recycling systems Ltd. (2010). ATS Technology Compost. Toronto, Ontario, Canada.

Patel, M., Ou, M. S., Ingram, L. O., \& Shanmugam, K. T. (2005). Simultaneous saccharification and co-fermentation of crystalline cellulose and sugar cane bagasse hemicellulose hydrolysate to lactate be thermotolerant acidophilic Basillus sp. Biotechnology Progress, 21(5), 1453-1460.

Parawira, W. \& Tekere, M. (2011). Biotechnological strategies to overcome inhibitors in 
lignocellulose hydrolysates for ethanol production: review. Critical Reviews in Biotechnology, 31(1), 20-31.

Percy, B. (2009). The performance of clostridium phytofermentans for biofuels production from lignocellulosic biomass (Master’s thesis). Ryerson University, Civil Engineering Dept., Toronto, Canada.

Philippidis, G. P., Sprindler, D.D., \& Wyman, C. A. (1992). Mathematical modeling of cellulose conversion to ethanol by the simultaneous saccharification and fermentation process. Applied Biochemistry and Biotechnoly, 34-34, 543-556.

Picataggio, S., Zhang, M., \& Filkenstein, M. (1994). Development of genetically engineered microorganisms for ethanol production. In M.E. Himmel, J.O. Baker and R.A. Overend (Eds.), In enzymatic conversion of biomass for fuel production ACS Symposium 566 Series (pp. 342-362). Washington, DC: American Chemical Society.

Reifenberger, E., Boles, E., \& Ciriacy, M. (1997). Kinetic characterization of individual hexose transporters of Saccharomyces serevisiae and their relation to the triggering mechanisms of glucose repression. European Journal of Biochemistry, 245(2), 324-333.

Retka, S.S. (2009). Genencor, novozymes launch new ethanol enzymes. Ethanol Producer Magazine. Retrieved from: http://ethanolproducer.com/article.jsp?article_id=5466.

Roberts, J. J., Cassula, A. M., Prado, P. O., Dias, R. A., \& Balestieri, J. A. P. (2015). Assessment of dry residual biomass potential for use as alternative energy source in the party of General Pueyrredón, Argentina. Renewable and Sustainable Energy Reviews, 41, 568583

Rogers, P.L., Joachimsthal, E.L., \& Hagget, K.D. (1997). Ethanol from lignocellullosics: potential from a Zymomonas-based process. Australasian Biotechnology, 7(5), 304-309. 
Rollin, J.A., Zhu, Z., Sathisuksanoh, N., \& Zhang, Y.-H. (2011). Increasing cellulose accessibility is more important than removing lignin: a comparison of cellulose solventbased lignocelluloses fractionation and soaking in aqueous ammonia. Biotechnology and Bioengineering, 108, 22-30.

Ruiz, R., \& Ehrman, T. (1996). Chemical analysis and testing laboratory analytical procedures.Determination of carbohydrates in biomass by high performance liquid chromatography. Lap-002. Technical report. Golden, Colorado, USA: National Renewable Energy Laboratory.

Sathitsuksanoh, N., Zhu, Z., Templeton, N., Rollin, J., Harvey, S., \& Zhang, Y.-H.P. (2009). Saccharification of a potential bioenergy crop, Phragmites australis (common reed), by lignocelluloses fractionation followed by enzymatic hydrolysis at decreased cellulase loadings. Industrial and Engineering Chemistry Research, 48, 6441-6447.

Sathitsuksanoh, N., Zhu, Z.G., Wi, S., \& Zhang, Y.-H.P. (2011). Cellulose solvent-based biomass pretreatment breaks highly ordered hydrogen bonds in cellulose fibers of switchgrass. Biotechnology and Bioengineering, 108, 521-529.

Schell, D. J., Farmer, J., Newman, M., \& McMillan, J. D. (2003). Dilute sulfuric acid pretreatment of corn stover in pilot scale reactor Applied Biochemistry and Biotechnology, 105-108,69-84.

Sedlak, M., \& Ho, N.W-Y. (2004). Characterization of effectiveness of hexose transporters for transporting xylose during glucose and xylose co-fermentation by a recombinant Saccharomyces yeast. Yeast, 21, 671-684. 
Selig, M.J., Viamajala, S., Decker, S.R., Tucker, M.P., Himmel, M.E., \& Vinzant, T.B. (2007). Deposition of lignin droplets produced during dilute acid pre-treatment of maize stems retards enzymatic hydrolysis of cellulose. Biotechnology Progress, 23(6), 1333-1339.

Shao, X. (2007). Cellulosic biomass to ethanol: kinetic modeling, scale up and reactor design (PhD thesis). Dartmouth College, Hanover, New Hampshire, USA.

Shao, X., Lynd, L., \& Wyman, C. (2009). Kinetic modeling of Cellulosic biomass to ethanol via simultaneous saccharification and fermentation. Part II. Experimental validation using waste paper sludge and anticipation of CFD analysis. Biotechnology and Bioengineering, 104(5), 932-938.

Shen, F., Hu, J., Zhong, Y., Liu, M. L.Y., Saddler, J. N., and Liu, R.(2012). Ethanol production from steam-pretreated sweet sorghum bagasse with high substrate consistency enzymatic hydrolysis. Biomass and Bioenergy,41, 157-164.

Sims, R. E. (2004). Bioenergy options for a cleaner environment: in developed and developing countries. San Diego,USA: Elsevier Science.

Sluiter, A., Hames, B., Ruiz, R., Scarlata, J., Templeton, D., \& Crocker, D. (2006). Determination of structural carbohydrates and lignin in biomass. Laboratory analytic procedure LAP-002.Technical Report. Colorado, USA: National Renewable Energy Laboratory. Retrieved from: http://devafdc. nrel.gov/pdfs/9572.pdf.

Skoog, K., \& Hahn-Hagerdal, B. (1990). The effect of oxygenation on xylose fermentation by Pichia stipitis. Applied and Environmental Microbiology, 56, 3389-3394.

South, S.R., Hogsett, D., \& Lynd, L. (1995). Modeling simultaneous saccharification and fermentation of lignocellulose to ethanol in batch and continuous reactors. Enzyme and Microbial Technology, 17, 797-803. 
Spindler, D. D., Wyman, C., Grohmann, K., \& Philipidis, G. P. (1992). Evaluation of the cellbiose-fermenting yeast brettanomyces-custers II in the simultaneous saccharification and fermentation of cellulose. Biotechnology Letters,14(5),403-407.

Sreenath, H. K., \& Jeffries, T. W. (2000). Production of ethanol from wood hydrolyzate by yeast. Bioresource Technology, 72, 253-260.

Stigler, S.M. (1986). The history of statistics. The measurement of uncertainty before. Cambridge, MA: Belknap Press of Harvard University.

Taherzadeh, M. J., \& Karimi, H. (2007). Acid based hydrolysis processes for ethanol form lignocellulosic materials. A review. Bioresorces, 2, 472-499.

Taherzadeh, M.J., \& Karimi, K. (2008). Pretreatment of lignocellulosic wastes to improve ethanol and biogas production: A review. International Journal of MolecularSciences, 9, 1621-1651.

Taherzadeh, M. J., Niklasson, C., \& Liden, G. (1999). Conversion of dilute acid hydrolyzates of spruce and birch to ethanol by fed-batch fermentation. Bioresource Technology, 69, 5966.

Tsuji, M., Singh, S. M., Yokota, Y., Kudoh, S., \& Hoshino, T. (2013). Influence of initial pH on ethanol production by the Antarctic Basidiomycetous yeast Mrakia blollopis. Bioscience, Biotechnology and Biochemistry, 77(12), 2483- 2485

U.S. Department of Energy.(1998). Annual energy review. Rep. DOE (IAE)-0384(97).US Department of Energy Office of Science and Office of Energy Efficiency and Renewable Energy. 
U.S. Department of Energy. (2006). Breaking the biological barriers to cellulosic ethanol: A joint research agenda, DOE/SC- 0095. US Department of Energy Office of Science and Office of Energy Efficiency and Renewable Energy.

U.S. Energy Information Administration.(2010).Gasoline and diesel fuel update.Washington DC: U.S. Department of Energy. Retrieved from: http://www.eia.doe.gov/oil_gas/petroleum/data_publications/wrgp/mogas_home_p age.html

U.S. Energy Information Adiministration (2014). Gasoline and diesel fuel update.Washington DC: U.S. Department of Energy. Retrieved from: http://www.eia.doe.gov/oil_gas/petroleum/data_publications/wrgp/mogas_home_page.ht $\mathrm{ml}$

van Uden, (1989). Alcohol toxity in yeasts and bacteria.Boca Raton, Florida: CRC Press. Inc. Vartek Waste Management Ltd. (2005). Vartek ATS Technology compost pilot test. Toronto, Ontario, Canada: Vartek Waste Management Ltd.

Wang, Q.Q., Zhu, J.Y., Hunt, C.G., \& Zhan, H.Y. (2012). Kinetics of adsorption, desorption, and re-adsorption of a commercial endoglucanase in lignocellulosic suspensions. Biotechnology and Bioengineering, 30(30), 124-132.

Wiselogel, A., Tyson, S., \& Johnson, D. (1996). Biomass feedstock resources and composition. In C.E. Wyman, M., Taylor, \& J. Francis (Eds). Handbook, production and utilization (pp. 105-118).Washington, DC: Taylor \& Francis.

Wright, J. D. (1998). Etanol from biomass by enzymatic hydrolysis. Chemical Engineering Progress,84(8), 62-74. 
Wyman, C. E. (1999). Biomass ethanol: Technical progress, opportunities, and commercial challenges. Annual Review Energy Environment, 24, 189-226.

Wyman, C.E., Dale, B.E., Elander, R.T., Holtzapple, M., Ladisch, M., \& Lee, Y.Y. (2005). Coordinated development of leading biomass pretreatment technologies. Bioresource Technology, 96, 1959-1966.

Wyman, C.E., \&Yang, B. (2009). Cellulosic biomass could help* meet California’s transportation fuel needs. California Agriculture, 63, 4, 185-190

Xiao, Z., Zhang, X., Cregg D.J., \& Saddler, NJ. (2004). Effects of sugar inhibition on cellulases and $\beta$-glucosidase during enzymatic hydrolysis of softwood substrates. Applied Biochemistry and Biotechnology, 115(1-3), 1115-1126.

Zhang, J. (2008). Simultaneous saccharification and co-fermentation of paper sludge (PhD thesis). Thayer School of Engineering, Datrmouth College, Hanover, New Hampshire.

Zhang, J., \& Lynd, L.R. (2010). Ethanol production from paper sludge by simultaneous saccharification and co-fermentation using recombinant xylose fermenting microorganisms. Biotechnology and Bioengineering, 107(2), 235-244.

Zhang, J., Shao, X., Townsend, O.V., \& Lynd, L.R. (2009). Simultaneous saccharification of paper sludge to ethanol by Saccharomyces cerevisiae RWB222.Biotechnology and Bioengineering, 104(5), 920-931.

Zhang, M., Chou, Y., Piccatagio, S., \& Finkelstein, M. (1998). Single Zymomonas mobilis strain for xylose and arabinose fermentation. Paper presented at Midwest Research Institute, Kansas, US. 
Zhang, M., Eddy, S., Deanda, K., Finkelstein, M., \& Piccatagio, S. (1995). Metabolic engineering of a pentose metabolism in ethanologenic Zymomonas mobilis.Science, 267, 240-243.

Zhang, M., Franden, M.A., Newman, M., McMillan, J., Filkenstein, J., \& Picataggio, S. (1995). Promising ethanologenes for xylose fermentation. Scientific note. Applied Biochemical Biotechnology, 51(52), 527-536.

Zhang, Y.-H.P., Cui, J-B., Lynd, L.R., \& Kuang, L.R. (2006). A transition from cellulose swelling to cellulose dissolution by o-phosphoric acid: Evidences from enzymatic hydrolysis and supramolecular structure. Biomacromolecules, 7(2), 644-648.

Zhang, Y.-H.P., Ding, S.-Y., Mielenz, J.R., Elander, R., Laser, M., Himmel, M., McMillan, J.D., \& Lynd, L.R. (2007). Fractionating recalcitrant lignocelluloses at modest reaction conditions. Biotechnology and Bioengineering, 97, 214-223.

Zhao, X., Zhang, L.,\& Liu, D. (2012). Biomass recalcitrance. Part I: the chemical compositions and physical structures affecting the enzymatic hydrolysis of lignocellulose. Biofuels, Bioproducts, \& Biorefining,6, 465-482.

Zhu, Y., Lee. Y.Y., \& Elander, R. T. (2007). Conversion of aqueous ammonia treated corn stover to lactoc acid by simultaneous saccharification and co-fermentation. Applied Biochemical Biotechnology, 137(1),721-738.

Zhu, Z., Sathitsuksanoh, N, Vinzant, T, Schell, D.J., McMillan, J.D., \& Zhang, Y.-H.P. (2009). Comparative study of corn stover pretreated by dilute acid and cellulose solvent-based lignocellulose fractionation: Enzymatic hydrolysis, supramolecular structure, and substrate accessibility. Biotechnology and Bioengineering,103(4), 715-724. 